UNIVERSITY OF PATRAS

SCHOOL OF MEDICINE

DePARTMENT OF MEDICAL Physics

NATIONAL TECHNICAL UNIVERSITY OF ATHENS

FACULTY OF MECHANICAL ENGINEERING

NATIONAL TEChNICAL UNIVERSITY OF ATHENS

FACULTY OF ELECTRICAL AND COMPUTER ENGINEERING
InTERdePARTMENTAL Program of Postgraduate Studies

IN BIOMEDICAL ENGINEERING

\title{
MODELING AND SIMULATION \\ OF FILTERS AND DEVICES \\ FOR CONFORMAL RADIOTHERAPY
}

PH.D. THESIS

TATJANA IVANOVA

PATRAS, 2007 


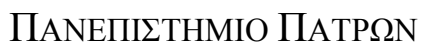

TMHMA IATPIKH $\Sigma$

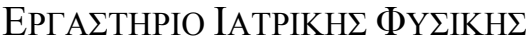

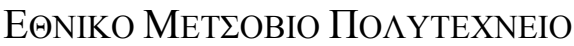

TMHMA MHXANO $\Lambda$ OГ $\Omega$ N MHXANIK $\Omega N$

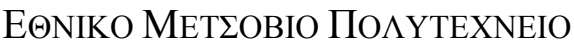

TMHMA H $\Lambda$ EKTPO $\Lambda$ OГ $\Omega$ N MHXANIK $\Omega N$

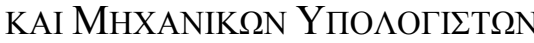

МІАТМНМАТІКО ПРОГРАММА

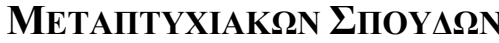

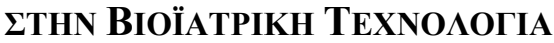

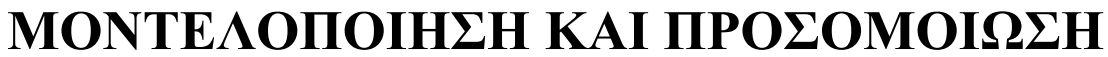 ФI $\Lambda T P \Omega N$ KAI $\Sigma Y \Sigma K E Y \Omega N$

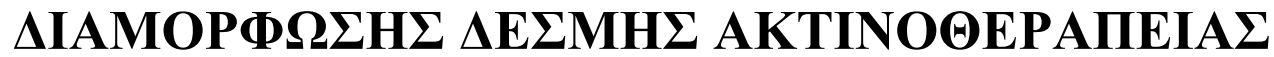

$\triangle \mathrm{I} \triangle \mathrm{AKTOPIKH} \triangle \mathrm{IATPIBH}$

TATJANA IVANOVA

ПАТРА, 2007 


\section{Three Members Advisory Committee}

Professor Nicolas Pallikarakis

Associated Professor Konstantinos Kappas

Dr. Zoi Kolitsi

\author{
Main Supervisor \\ Member of the Advisory Committee \\ Member of the Advisory Committee
}

\section{Seven Members Examination Committee}

Professor Nicolas Pallikarakis

Associated Professor Konstantinos Kappas

Associated Professor Panayiotis Dimopoulos

Associated Professor Dimitrios Kardamakis

Professor Dimitrios Koutsouris

Assistant Professor Eleni Kostaridou

Professor Georgios Panayotakis
Main Supervisor

Member of the Advisory Committee Member of the Examination Committee Member of the Examination Committee Member of the Examination Committee Member of the Examination Committee Member of the Examination Committee 


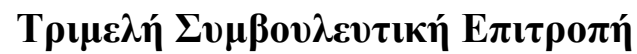

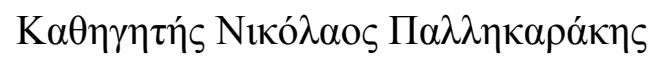

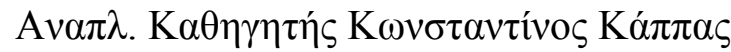

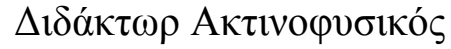

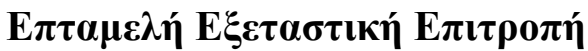

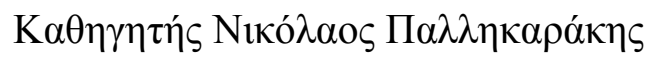

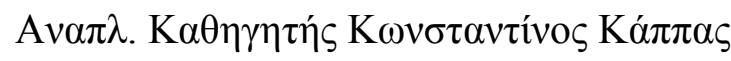

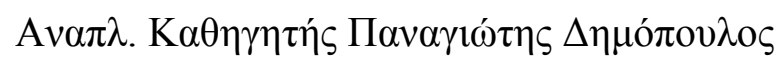

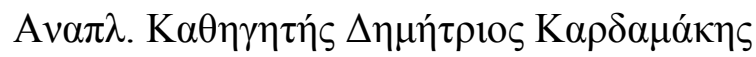

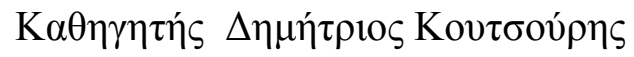

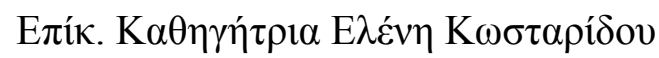

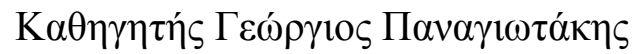

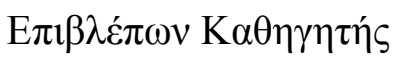

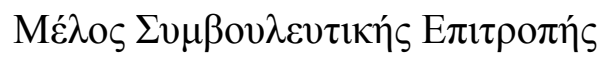

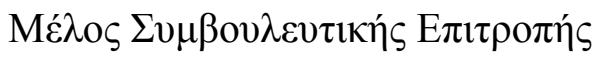

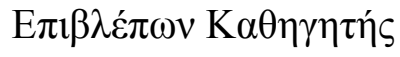

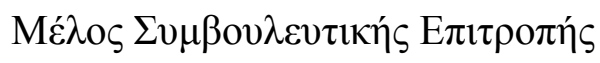

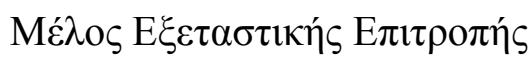

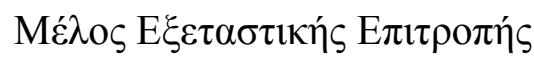

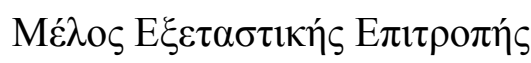

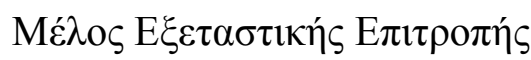

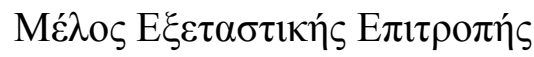




\begin{abstract}
This doctoral thesis addresses dynamic intensity modulation by means of physical beam modifiers. The principles of gravity-oriented devices were generalized and extended, preserving principles of a beam shaping, but introducing motor-driven "patient-oriented" beam modifying devices. Beam modifying devices were divided in two categories: protectors and shapers. The protectors are diminished copies of the Organs At Risk (OARs) and stay parallel to them during gantry rotation, keeping them in the attenuated field for every gantry angle. Shapers are placed at the both sides of the protector to ensure uniform dose in the Planning Target Volume (PTV).
\end{abstract}

Mathematical formalism for calculations of the dimensions and the initial coordinates of the beam modifying devices was developed as well as the laws of their motion during gantry rotation were derived. A software tool, incorporating the mathematical background, with user interface to facilitate the introduction of the input parameters was created. The software module was subsequently integrated into a Monte Carlo Radiation Therapy Simulator (MCRTS), used to simulate particle transport through the designed system.

Simulation studies of field shaping in rotational therapy by means of beam modifying devices were carried out. Dose distributions in solid-geometry and voxel-based neck models were evaluated. Furthermore, the effectiveness of the shapers to modify the dose distribution outside the protected area was studied. The results of simulation studies showed that rotational therapy with beam modifying devices offers adequate protection of the OAR and a uniform dose distribution outside the protected region. Studies using shapers of different materials were also carried out and resulted in similar dose distributions.

Additionally, the effect of protector's parameters on the dose distribution in rotational therapy was studied in the thesis. A range of materials, consisting of commonly used for protection in radiotherapy, as well as by some new metal-polymer composites was under investigation. The results of studies in rotational therapy have shown that a protector of high density and bigger diameter offers more extensive protection of the OAR. In the case of cylindrical protector, however, increase in diameter leads to increase of projected shadow, which is not always desirable. So, the solution can be to use larger diameter protector, placed closer to the phantom. Scatter contribution to the center of the OAR from protector is negligible. Surface dose is increasing not more than by $1 \%$ of the dose of the open beam. Moreover, steeper dose increase beyond the protected region is achievable with higher density protectors, placed further from the irradiation source. The metal-polymer composites can rival the lead in the protection of vital organs if the density provided is high.

Some aspects of the MCRTS were verified in the work by means of comparison of the results of simulations with literature and measurements data.

The presented technique has showed promising results in terms of conformal dose delivery and can be a preferred choice in radiotherapy departments due to comprehensive and adequate protection of the OAR and uniform dose in PTV ensured as well as of its cost effectiveness. 


\section{ПЕРІАНЧН}

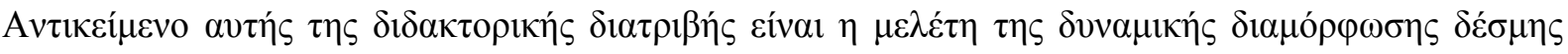

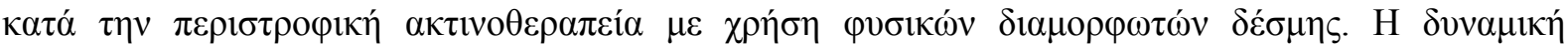

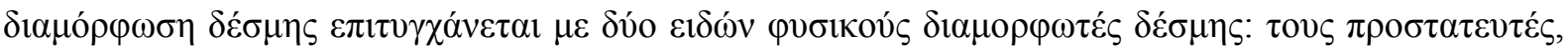

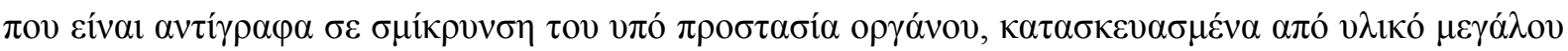

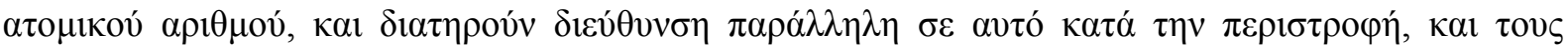

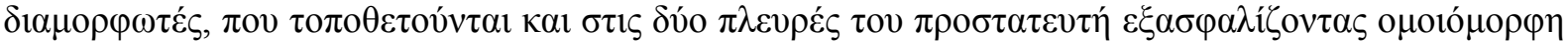

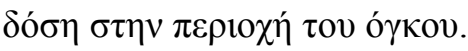

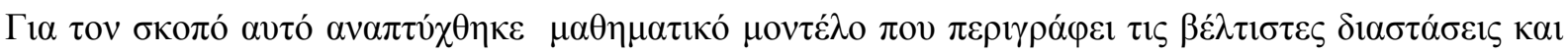

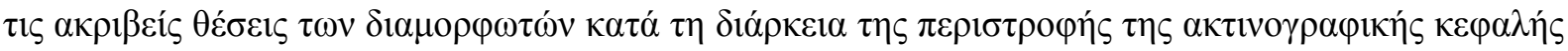

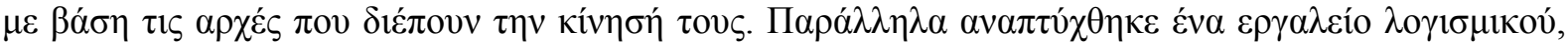

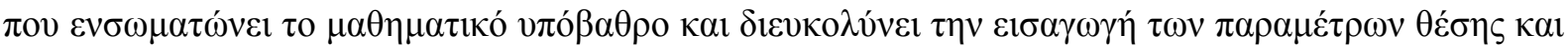

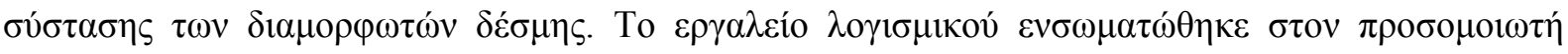

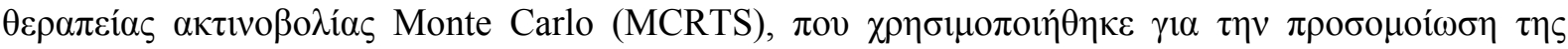

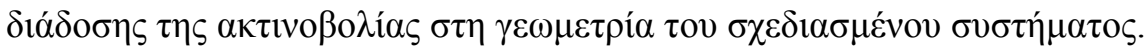

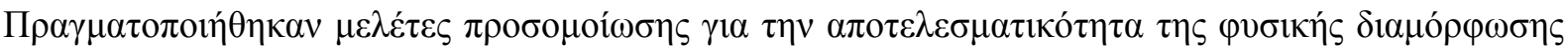

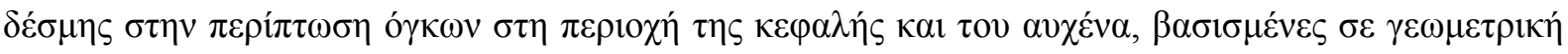

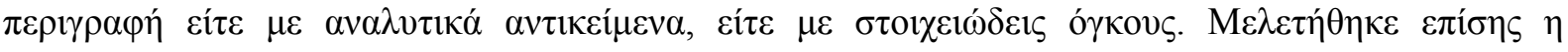

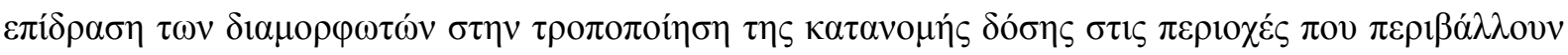

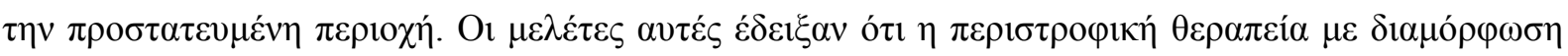

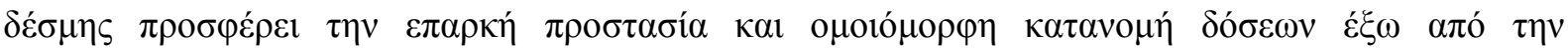

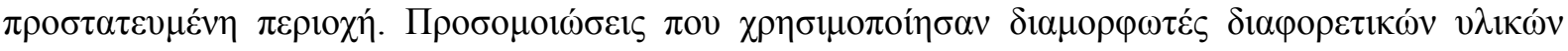

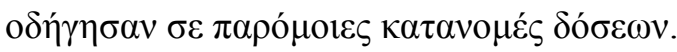

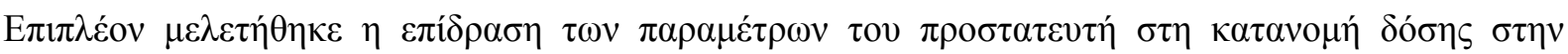

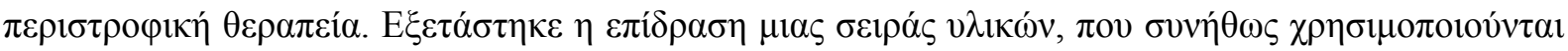

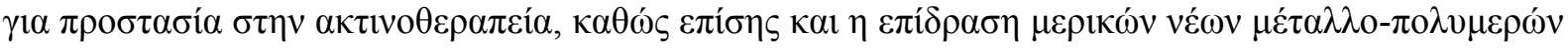

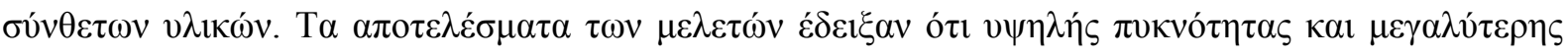

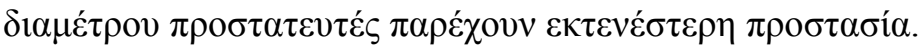

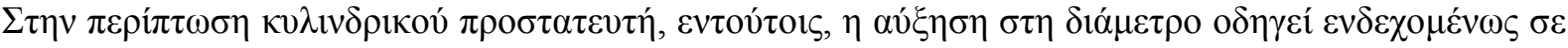

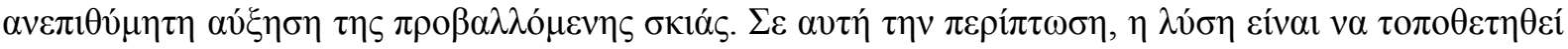

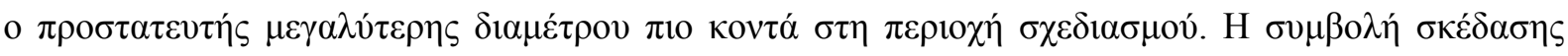

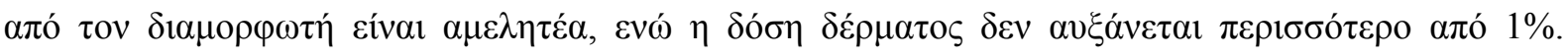

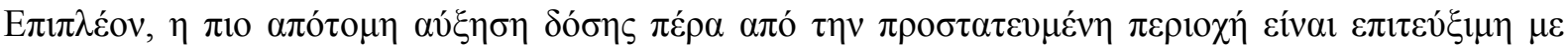

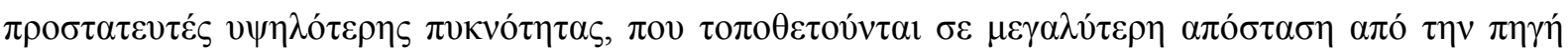

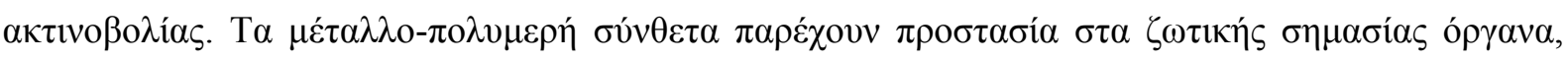

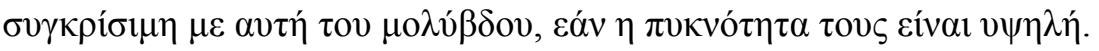

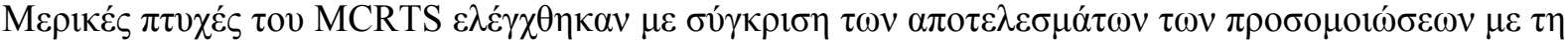

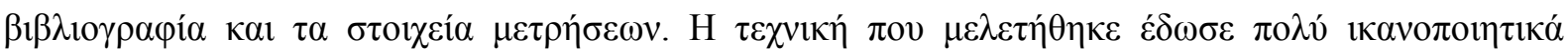

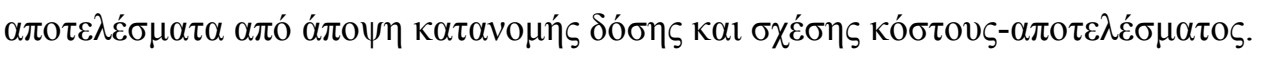




\section{PUBLICATIONS}

\section{International peer-reviewed journals:}

T. Ivanova, K. Bliznakova and N. Pallikarakis, "Simulation studies of field shaping in rotational radiation therapy”, Med. Phys. 2006, 33: 4289-4298.

T. Ivanova, G. Malatara, K. Bliznakova, D. Kardamakis and N. Pallikarakis, "The effect of the attenuators properties on the dose distributions in rotational radiation therapy", Radiother. Oncol. (submitted)

\section{Conferences proceedings:}

T. Ivanova, G. Malatara, K. Bliznakova, D. Kardamakis and N. Pallikarakis, "Studies on the attenuating properties of various materials used for protection in radiotherapy and their effect of on the dose distribution in rotational therapy", 11th Mediterranean Conference on Medical and Biological Engineering and Computing, MEDICON 2007, Ljubljana, Slovenia, June 26-30, 2007 (submitted)

T. Ivanova, K. Bliznakova and N. Pallikarakis, "Simulation studies on the effect of protectors on dose distribution in rotational radiotherapy", Proceedings of the 5th European Symposium on Biomedical Engineering, Patras, Greece, July 7-9, 2006 (oral and poster presentations).

T. Ivanova, K. Bliznakova, N. Pallikarakis: "Studies of field shaping with "omoiotheta" beam modulators in rotational radiation therapy”, IFMBE Proceedings (2005), Volume 11(1), p. 1714-1717, The 3rd European Medical \& Biological Engineering Conference EMBEC’05, Prague, November 2025, 2005 (poster presentation).

T. Ivanova and K. Bliznakova: "Intensity modulation studies with "omoiotheta” beam modulators in rotational radiotherapy”, Book of Abstracts P87, p. 212, 1st Conference on Biosciences of University of Patras, Patras, Greece, May 19-20, 2005 (poster presentation).

T. Ivanova, K. Bliznakova: "Development and verification of a software module for simulation of filters in conformal radiotherapy", Proceedings of the 4th European Symposium on Biomedical Engineering, Patras, Greece, June 25-27, 2004 (oral and poster presentations). 


\section{ACKNOWLEDGEMENTS}

The Department of Medical Physics of University of Patras and Greek State Foundation of Scholarships made possible my doctoral studies in Biomedical Engineering and I would like to express my gratitude to this institutions.

I would like to take this opportunity to thank everybody who helped me to complete this research work. My sincere thanks go to my advisor Professor Nicolas Pallikarakis for the guidance of the work that leaded to the present doctorate thesis. Without his support this work would have been impossible. I would like also to thank Associate Professor Constantinos Kappas and Dr. Zoi Kolitsi for their participation in my advisory committee.

I'm very thankful to Dr. Kristina Bliznakova for her assistance, collaboration and support during the whole research work.

My gratitude is also addressed to the members of the Department of Medical Physics, University Hospital of Larissa, especially to Assistant Professor Kiki Theodorou, for their hospitality and useful knowledge and experience that I got during my visit to the department.

My sincere thanks I'd like to express to Professor Basil Proimos and Dr. Claudia Danciu for the fruitful discussions and assistance on some points of this work.

I would like also to thank Dr. Zetta Malatara and Associate Professor Dimitrios Kardamakis for their collaboration and performing measurements at the Department of Radiation Therapy, University Hospital of Patras as well as for their support in providing spectral data.

I would like sincerely to thank Dr. Dimitris Lazos for fruitful discussions and his useful opinions during this work.

And last but not least, I would like to thank my mother for her patience and 133 letters received from her during my Ph. D. studies in Greece. 


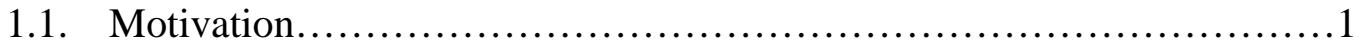

1.2. Studied problems..............................................

1.3. Thesis outline..........................................................

$\begin{array}{lll}\text { CHAPTER } 2 & \text { Three-dimensional conformal radiation therapy } & 7\end{array}$

2.1. Introduction....................................................

2.2. Intensity modulation with physical compensators......................8

1.2.1. One-dimensional beam intensity modifying devices wedges.....................................................8

1.2.2. Two-dimensional beam intensity modifying devices blocks and compensators..................................9

2.3. Tomotherapy ................................................... 14

2.4. IMRT with MLC ............................................... 17

2.5. Alternative IMRT methods .....................................22

2.6. Conclusions: for and against IMRT ................................24

CHAPTER 3: Inverse planning problem in radiation therapy 27

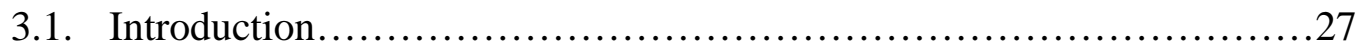

3.2. Direct inverse problem..........................................27

3.2.1. Dose distribution of radial symmetry...................28

3.2.2. Dose distribution of reflection symmetry..................33

3.2.3. Arbitrary dose distribution .............................35 
3.3. Similarity of inverse problem in radiation therapy with image reconstruction in CT............................................40

3.4. Going beyond the direct inverse problem ............................45

3.5. Conclusions.......................................................48

CHAPTER 4: Rotational conformal radiotherapy with beam modifying devices 51

4.1. Introduction................................................. 51

4.2. Principles of the beam modifying devices...........................53

4.3. Mathematical modeling of the beam modifying devices.................57

4.3.1. Protectors...............................................57

4.3.2. Beam shapers......................................59

4.4. Discussion and conclusions........................................64

CHAPTER 5: Simulations studies on use of the beam modifying devices for spinal cord protection

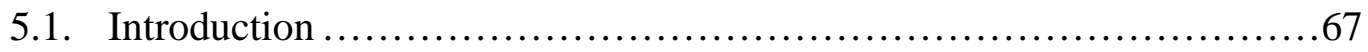

5.2. Methods and materials.............................................68

5.2.1. The Monte Carlo simulator............................68

5.2.2. The software tool for calculations of beam modifying devices ...................................70

5.2.3. The spectra of irradiation used ..........................71

5.2.4. Neck models and irradiation setups.....................71

5.2.5. Simulations with solid-geometry neck model .............74

5.2.6. Simulations with voxel-based neck model ................80

5.3. Results ........................................................... 82

5.3.1. Verification of the software module.....................82

5.3.2. Studies of the dose distributions acquired using the beam modifying devices................................83

5.3.3. Assessment of the shapers' parameters calculation methods.................................87

5.3.4. Application study with the voxel-based neck model........90

5.4. Discussion and conclusions.........................................91

\section{CHAPTER 6: Studies on the effect of protectors on dose distributions} in a homogeneous phantom

6.1. Introduction.....................................................95

6.2. Theoretical background.........................................97

6.3. Materials and methods..............................................98

6.3.1. Choice of the materials appropriate for beam modifiers manufacturing...............................98

6.3.2. Measurements of beam attenuation by different materials............................................100

6.3.3. Simulation studies for MCRTS verification...............102 
6.3.4. Evaluation and comparison of the simulated and measured data ..........................................103

6.3.5. Simulation studies of rotational irradiation ................104

6.4. Results ........................................................ 108

6.4.1. Verification studies...................................108

6.4.2. Measurements with $18 \mathrm{MV}$ beam attenuation..............111

6.4.3. Simulation studies with cylindrical protectors ............112

6.5. Discussion and conclusions ....................................124

CHAPTER 7: Conclusions and future work directions 131

$\begin{array}{ll}\text { Bibliography } & 135\end{array}$

$\begin{array}{ll}\text { Acronyms } & 145\end{array}$

Appendices

Appendix A................................................................

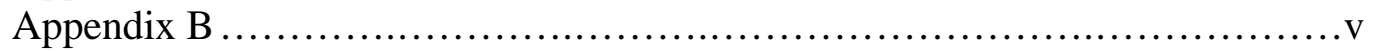


INTRODUCTION

\subsection{MOTIVATION}

Radiation therapy is the use of ionizing radiation for the complete destruction of an entire tumor or the shrinking of a tumor as a means of symptom relief. More specifically, the goal of the Three-Dimensional Conformal Radiation Therapy (3D-CRT) is to deliver a suitable treatment dose to the target volume without exceeding the tolerance dose of the adjacent healthy tissue and organs. The delivery of the desired dose pattern, especially to the complex in shape Planning Target Volume (PTV), can be achieved most efficiently by the beam intensity modulation. Intensity-Modulated Radiation Therapy (IMRT) is a delivery of radiation to the patient via fields that have non-uniform radiation fluence (Webb 2003). The way and the hardware of the techniques employed may vary. The devices used for delivery can be divided into 4 major categories (Galvin 2006): (1) binary Multileaf Collimators (MLCs), (2) standard MLCs, (3) relatively small circular collimators, and (4) traditional adjustable jaw collimators coupled with machined compensators that are inserted at the front face of the linear accelerator head.

IMRT is rapidly evolving during the recent years. The rate of clinical acceptance increases both in the US and worldwide. According to a survey done in 2005, 73\% of the 
responding US radiation oncologists used IMRT clinically in 2004, while in 2002 the number was 32\% (Mell et al. 2005). However, the complexity and cost of implementing and maintaining these new technologies makes them inaccessible for small radiotherapy departments. The quick spread of IMRT in US centers has undoubtedly been fuelled by a favorable reimbursement for this procedure (Bentzen 2005). Likewise, the slower uptake in European centers reflects the difficulties in introducing major new technologies in departments that are under considerable service pressure. There is a discussion about if these sophisticated techniques are necessary for routine use and if the achieved dose elevation indeed increases a patient's lifetime (Schulz 1999, Schulz 2001, Schulz and Kagan 2002). IMRT may be used, however, to intensify treatment or/and reduce treatment toxicity (Bentzen 2005). Without doubts, it improves the quality of life of the patient as the sensitive adjacent to the tumor tissues can be spared (Jabbari et al. 2005). Techniques using both compensators and MLCs have inherent advantages and disadvantages, with the common objective to deliver a homogeneous dose to the tumor within $\pm 5 \%$ (ICRU report 50, 1993) of the dose prescription and to keep the dose to the Organs At Risk (OAR) below a certain value.

In radiation therapy of well defined deep seated tumor volumes, rotational therapy is often a method of interest (Brahme et al. 1982). However, a circular dose distribution may not be suitable for a target enveloping or abutting a sensitive structure. To produce more complicated dose distributions with rotational therapy requires a beam intensity that may vary with both the distance from the center of the field and the gantry angle (Cormack 1998). The technique of gravity-oriented devices that contains these properties, showed very promising results in terms of the OAR protection and the dose uniformity in the PTV (Proimos 1966, Danciu 2001). Moreover, the device is compatible to any linear accelerator and uses inexpensive materials. Also, there is no need to change the filter for each gantry position.

For any radiotherapy department accessible and effective cancer treatment techniques are of great importance. The technique should be able to deliver a treatment according to the definition of the conformal radiotherapy. Moreover, it should include practicality, easiness in exploitation, simple quality assurance and low expenses. So, comprehensive studies of the conformal dose delivery by means of beam modifying devices, based on the principles of gravity-oriented devices, were carried out in this thesis and can be a step forward towards an application of this technique. 


\subsection{STUDIED PROBLEMS}

The scope of this thesis is in the area of the IMRT with dynamic intensity modulation by means of physical beam modifiers. This study had the following objectives:

(1) To generalize the idea of intensity modulation by gravity-oriented filters and to introduce similar by properties beam modifying devices.

The beam modifying devices for rotational radiotherapy based on the principles of gravity- oriented devices, but oriented arbitrarily, or more specifically- "treatment-oriented", with subsequent motion during the gantry rotation driven by a motorized system were introduced in the thesis. The beam modifying devices were divided in two categories: protectors and beam shapers. During gantry rotation the protector, which is a diminished copy of the OAR, rotates so that its cast shadow coincides with the OAR at any gantry position, thus significantly reducing the primary radiation. A method for calculations of the geometrical parameters of the protector was worked up. The achieved dose reduction at the central axis under the attenuator was evaluated analytically. A part of the volume behind the protector stays in the shadow for every gantry angle, thus the dose at the PTV is reduced. Moreover, the closer the region is to the OAR, the larger dose reduction is observed and the dose outside the OAR is non-uniform. To eliminate this nonuniformity, additional beam shapers have to be used. The mathematical formalism for derivation of the initial coordinates of the beam shapers and the laws of their motion during the gantry rotation were developed. Two different approaches for geometrical and physical shapers’ parameters calculations were developed and substantiated with later verification of the methods.

\section{(2) To develop a software tool for calculations of the parameters of beam modifying devices.}

To facilitate a modeling of beam modifying devices and their further integration into the simulation scheme, a new user interface was created. A software tool that incorporates the developed mathematical background for calculations of the initial coordinates and geometrical parameters was integrated into the in-house-developed Monte Carlo Radiation Therapy Simulator (MCRTS) (Bliznakova et al. 2004), used for simulation studies in this work. 


\section{(3) To perform simulation studies on the use of the beam modifying devices in rotational radiation therapy.}

Protection the spinal cord with the target volume to be irradiated located near it was chosen for a case study. Simulation studies of field shaping by different configurations of the beam modifying devices, using ideal (solid-geometry) and real (voxel-based) phantoms, were carried out. The degree of protection of OAR and the quality of dose distribution outside the protected region in terms of uniformity were evaluated.

\section{(4) To investigate the effect of geometrical and physical parameters of the protector on the dose distribution in rotational therapy.}

Experimental measurements of the beam attenuation by a number of materials, new at the market and traditionally used in radiation therapy for a protection of vital organs, were carried out. For more sophisticated studies of the effect of the protector's parameters on the dose distribution in rotational therapy the Monte Carlo simulations were employed. The dose distribution is a function of many parameters, which are both characteristics of the irradiation beam and of the attenuator (El-Khatib et al. 1988). Therefore, the extent of the influence of such parameters of the cylindrical protectors as diameter, its distance from the isocenter, density as well as field size on the dose distribution was studied. The materials, used as the beam absorbers, consisted of lead, tungsten, gold, Cerrobend and metal-polymer composite material Rad-block. Central dose profiles of the resultant dose distributions were evaluated as well as doses at the center of the OAR, phantom surface doses, ratios of the dose at the center of the OAR to the maximal dose at the PTV, and dose gradients just beyond the OAR.

The approaches adopted to solve the above defined tasks included both simulations and experimental studies. The MCRTS was used for three-dimensional dose distribution computations in various phantoms in the simulation studies. The ability to simulate radiotherapy treatments using Monte Carlo method facilitates the calculation of accurate dose distributions. Measurements of the beam attenuation in different materials were employed for studies of attenuating properties of a range of materials. The results of the measurements were used as a benchmark for a comparison with simulated data.

In the process of the work, some special aspects of the MCRTS were verified by a comparison of the results of simulations with published data or with the results of the 
measurements before carrying out the simulation studies for receiving reliable and trustworthy final results.

\subsection{THESIS OUTLINE}

A discussion starts, in Chapter 2, with the review of IMRT techniques. The techniques were classified according to the hardware that is used for the intensity modulation: IMRT with physical compensators, tomotherapy, IMRT with MLC, including Intensity-Modulated Arc Therapy (IMAT). The subchapter “Alternative IMRT methods” refers to some pilot studies that are not commonly accepted by the radiotherapeutic community or belong to the prototypes being under investigations. The historical survey and the general description of the gravity-oriented devices are also included with more detailed description of the principles of operation in the introduction of the Chapter 4. Chapter 2 concludes with the comparison of the presented IMRT techniques between themselves and the discussion about advantages and disadvantages of the present treatment techniques in radiotherapy.

Chapter 3 is devoted to the review of the historical evolution of the inverse planning in radiotherapy and to the reasoning to what extend it can be applied. Inverse problem in radiotherapy answers the question (Brahme et al. 1982): "Which should be a lateral intensity profile in the incident beam that produces a desired absorbed dose distribution?” Historically, first analytical solutions for simple problems in rotational therapy were found (direct inverse planning). Because of some difficulties encountered in an analytical solution of the inverse problem, such as negative fluencies, symmetry considerations, infinite number of solutions, unachievable dose distributions, the definition of the desired dose distributions as well as the calculation methods were altering. Contemporarily, inverse planning uses the concept of objective function for the optimization of the inverse problem. A subject of similarity of the inverse problem in radiotherapy and CT image reconstruction task as well as their differences, such as negative fluence impossibility in RT were touched upon. The ways of overcoming this unwanted feature were discussed.

In Chapter 4, a mathematical description of motion of the beam modifying devices and the formalism for the calculations of their parameters was developed. The beam modifying devices were divided in two categories in this work: protectors and shapers. The protectors used are diminished copies of the Organs At Risk and stay parallel to them during gantry rotation. Thus, each protector always keeps the corresponding OAR in its shadow, 
significantly reducing the irradiation. The shapers are located at both sides of the protector and are used to obtain a more uniform dose distribution in the PTV. They preserve their initial orientation during gantry rotation. Two shapers parameters calculations methods were developed: “for diameter to material” and vice verse.

Chapter 5 is dedicating to the simulation studies on the use of the beam modifying devices for the spinal cord protection. In order to facilitate the computations of the geometrical setup of the beam modifying devices, a software application has been developed. A software module, incorporating the above stated mathematical background was further integrated into the MCRTS for direct introduction of the geometrical and physical parameters of the beam modifying devices into the simulation scheme. In this chapter the following tasks were implemented, namely: (1) the software module for calculation of the parameters and initial coordinates of the beam modifying devices was verified; (2) the methods of shapers calculations were verified, as well, and (3) simulation studies of field shaping by different configurations of the beam modifying devices, using solid-geometry and voxel-based phantoms, were carried out and the degree of protection of OAR and the quality of dose distribution outside the protected region in terms of uniformity were evaluated.

Chapter 6 deals with the studies of the effect of the protector's parameters on the dose distribution in a homogeneous phantom. Measurements of beam attenuation by materials commonly used for protection in radiotherapy, such as lead, cerrobend and brass, as well as by three new materials for radiation shielding, which are polymers filled with tungsten powder, were carried out. The results of the measurements were compared with the results of the simulations for the same experimental setup to verify some aspects of the MCRTS. The later was also used for simulation studies on the effect of cylindrical protectors on the dose distributions in the rotational radiotherapy. The quality of the received dose distributions was evaluated in terms of dose at the center of the OAR, phantom surface dose, the ratio of the dose at the center of OAR to the maximal dose at the PTV and the dose gradient outside the OAR

The thesis ends with a chapter devoted to concluding remarks and future work directions 
CHAPTER

\section{THREE-DIMENSIONAL CONFORMAL RADIATION THERAPY}

\subsection{INTRODUCTION}

The development and clinical use of three-dimensional conformal radiation therapy (3D-CRT) is a major research area in the field of radiation oncology in the recent years. The goal of 3D-CRT is to deliver a suitable treatment dose to the target volume without exceeding the tolerance dose of the adjacent healthy tissue and organs (Fraass 1995). Classification of CRT depends upon the definitions of the constituents of CRT. Intensity Modulated Radiation Therapy Collaborative Working Group (IMRT-CWG 2001) uses subdivision into conventional 3D-CRT and new type of 3D-CRT- Intensity-Modulated Radiation Therapy (IMRT). In conventional 3D-CRT the delivery of the radiation is accomplished with a set of fixed or rotating radiation beams, shaped using the projection of the target volume. The radiation beams normally have a uniform intensity across the field, or, where appropriate have this intensity modified by simple beam fluence-modifying devices, such as wedges or compensating filters. IMRT is based on the use of non-uniform radiation beam intensities incident on the patient that have been determined using various computer-based optimization techniques. While Webb (2001) divides CRT in two classes: those, which employ geometric fieldshaping alone and those, which also modulate the intensity of fluence across the 
geometrically-shaped fields. Blocks, wedges, gravity-oriented devices and tissue compensators produce intensity-modulated distributions, so these techniques belong to IMRT, however, can be referred to the old CRT history. Modern history of IMRT starts with the concept of inverse planning firstly introduced by Brahme in 1982. Inverse planning creates from the ideal dose prescription the best set of intensity-modulated beams for the problem. The main branches of modern IMRT are intensity modulation by the use of cast compensator (Chang et al. 2004, Jiang and Ayyangar 1998, Weeks and Sontag 1990), tomotherapy (Mackie et al. 1993, Oldham and Webb 1997), IMRT with Multileaf Collimator (MLC), used either in a static (Bortfeld et al. 1994, Xia and Verhey 1998) or in a dynamic mode (Källman at al 1988, Convery and Rosenbloom 1992, Chui et al. 2001), including Intensity Modulated Arc Therapy (IMAT) (Yu 1995). The methods differ in their complexity and in the hardware necessary to deliver the radiation. Other methods of intensity modulation, reported in literature, are intensity modulation by means of gravity-oriented devices (Proimos 1962, Proimos 1966, Proimos et al. 1966), reshapable physical modulator (Xu et al. 2002), single computer-controlled absorber (Fiorino et al 1995), use of swept pencils of radiation (Brahme 1987), IMRT delivery by robotic linac (Webb 1999, Webb 2000) and multiportal compensation system (Yoda and Aoki 2003).

\subsection{INTENSITY MODULATION WITH PHYSICAL COMPENSATORS}

\subsubsection{One-dimensional beam intensity modifying devices- wedges}

Wedge filter is a wedge-shaped absorber, which causes a progressive decrease in the intensity across the beam, resulting in a tilt of the isodose curves from their normal positions (Khan 1994). The degree of tilt depends on the slope of the wedge filter. The wedge usually is made of dense material, such as lead or steel, and is mounted on a transparent plastic tray, which can be inserted in the beam at a specified distance from the source.

Although wedge primarily modify beam intensity in one dimension (along the wedge), dose profiles produced by wedge filters in the non-wedged direction can exhibit a $7 \%$ or greater dose reduction at the outer ends of the field compared with open beam profiles (Keall et al. 1997). This discrepancy increases with off-axis distance and wedge angle. This sagging is mainly due to the softening of the energy spectrum off-axis. The factors that contribute the off-axis softening are: photon attenuation to a greater extent than the central axis photons, 
increase of scattered photon fluence and increase in the path-length of the off-axis photons due to angulation of the beam. As open field profiles are used for the off-axis dose calculation by most planning computers, a method of construction of the wedge for a radiotherapy beam which reproduces the open beam profile shape in the non-wedge axis was proposed by Keall et al. (1997).

\subsubsection{Two-dimensional beam intensity modifying devices- blocks and compensators}

In conventional Radiation Therapy two kinds of beam modifiers are used: blocks and compensators. Blocks are usually made of high-density material of big thickness (see Table 21). Thickness of lead between 4.5-5 half-value layers (HVLs), that gives less than $5 \%$ of the primary beam transmission, is recommended for most clinical shielding. However, scattered radiation from the adjoining open areas of the field increases the dose beyond the block (up to15 to $20 \%$ ). The shaping of the treatment field is primarily dictated by the tumor distribution. The procedure of constructing custom blocks starts with a simulator radiograph or a port film on which the radiotherapist draws the outline of the treatment field indicating areas to be shielded. The film is then used to construct divergent cavities in a Styrofoam block by means of Styrofoam cutting device that is used to cast Cerrobend blocks (Khan 1994).

In radiation therapy, compensators can be used to fulfill various tasks, as it is shown in Table 2-1. Historically, compensation was implemented to correct for the irregularity of the surface of the patient. Later on, compensators started to be used for a uniform dose delivery at a given plane. The goal of contemporary IMRT compensator design is to produce an optimized primary fluence profile at the patient's surface obtained from the optimization procedure, to achieve dose uniformity throughout the whole target volume and to spare critical structures according to the dose and dose-volume constrains prescribed by the clinician for a specific patient (Jiang and Ayyangar 1998). Customized compensators are shaped to attenuate the open-field photon fluence such that the transmitted fluence map is as designed by the dose optimization algorithm (Chang et al. 2004).

The major difference between MLC or scanning pattern based IMRT and compensator based IMRT is that the introduction of the compensator inside a radiation field causes changes in beam characteristics other than intensity. Basically, the compensator induced beam perturbations include the hardening of the primary photon spectrum, beam contamination 
TABLE 2-1. Comparison of the properties of blocks and compensators used for intensity modulation.

\begin{tabular}{|c|c|c|c|c|}
\hline & \multicolumn{2}{|c|}{ BLOCKS } & \multicolumn{2}{|c|}{ COMPENSATORS } \\
\hline & narrow & wide & & \\
\hline Made of: & high densit & material & $\begin{array}{l}\text { high density } \\
\text { material }\end{array}$ & low density material \\
\hline \multirow[t]{3}{*}{ Functions: } & \multirow{3}{*}{\multicolumn{2}{|c|}{$\begin{array}{l}\text { - to shield (fully or partially) } \\
\text { certain sensitive structures of the } \\
\text { body }\end{array}$}} & \multicolumn{2}{|c|}{$\begin{array}{l}\text { - to deliver IMRT treatment designed by } \\
\text { optimization algorithms }\end{array}$} \\
\hline & & & & $\begin{array}{l}\text { - to compensate a } \\
\text { missing tissue in areas } \\
\text { where dose surface is } \\
\text { not flat }\end{array}$ \\
\hline & & & & $\begin{array}{l}\text { - to shape a dose } \\
\text { distribution inside the } \\
\text { body to improve dose } \\
\text { homogeneity }\end{array}$ \\
\hline Advantages: & $\begin{array}{l}\text { Avoidance of } \\
\text { miss of tumor } \\
\text { treatment dose }\end{array}$ & $\begin{array}{l}\text { Provide more } \\
\text { secure } \\
\text { protection }\end{array}$ & Small thickness & $\begin{array}{l}\text { The transmission of the } \\
\text { compensator is not } \\
\text { very sensitive to small } \\
\text { inaccuracies in } \\
\text { thickness }\end{array}$ \\
\hline $\begin{array}{l}\text { Dis- } \\
\text { advantages: }\end{array}$ & $\begin{array}{l}\text { Increase } \\
\text { variation in dose } \\
\text { across block } \\
\text { shadow and risk } \\
\text { of mispositioning }\end{array}$ & $\begin{array}{l}\text { Shield more } \\
\text { potential tumor } \\
\text { sites }\end{array}$ & $\begin{array}{l}\text { Greater sensitivity } \\
\text { to random and } \\
\text { systematic errors } \\
\text { in cutting or } \\
\text { assembling } \\
\text { compensator }\end{array}$ & $\begin{array}{l}\text { Small range of } \\
\text { intensity modulation } \\
\text { magnitude }\end{array}$ \\
\hline
\end{tabular}

from the compensator scattered photons, and the influence of the compensator on contaminant electron dose (Jiang and Ayyangar 1998).

Shragge and Patterson (1981) have created an iterative algorithm used to design compensators, which produce a uniform dose distribution in the plane of calculation. In the first iteration, a dose distribution is calculated by means of dividing the radiation into primary and scattered components by separating the Tissue-Air Ratios TARs into zero area TARs forthe primary radiation and SARs for scattered radiation. The maximum and the minimum doses are then found for the calculated points and error defined as ERROR $=\left(D_{\max }-D_{\min }\right) / D_{\max }$ is calculated. If ERROR is greater than the allowable error $E$, then a thickness of lead $t$ is added above each point in the plane according to

$$
\mathrm{t}=\frac{1}{\mu} \ln \left(\frac{\mathrm{D}(\mathrm{x}, \mathrm{y})}{\mathrm{D}_{\min }}\right)(\mathrm{cm}),
$$


where $\mu(\mathrm{cm}-1)$ is the beam attenuation coefficient the compensator material, $\mathrm{D}(\mathrm{x}, \mathrm{y})$ is the dose at position $(\mathrm{x}, \mathrm{y})$, and $\mathrm{D}_{\min }$ is the minimum calculated dose in the plane of calculation.

Jursinic et al. (1994) have calculated the required thickness of the compensator along a ray connecting a point in the plane of compensation to the source as follows:

$$
\mathrm{t}=\frac{1}{\mu} \ln \left(\frac{\mathrm{D}_{\text {actual }}}{\mathrm{D}_{\text {desired }}}\right),
$$

where $\mu$ is the narrow beam linear attenuation coefficient of the compensating material at the center of the beam. The improvements in compensator design can be accomplished by taking into account the off-axis beam energy dependence.

Another method of designing compensators for a single beam or one or more pairs of beams was presented by Mageras et al (1990). The design process employs convolution of Monte Carlo generated pencil beams with photon fluence distribution, appropriately modified for the presence of beam modifiers, to compute dose in a flat homogeneous phantom. Corrections for inhomogeneities and surface curvature are applied by using computerized tomography information to determine the effective path length through the tissue. Multiple iterations are used to arrive to a compensator that properly incorporates changes in radiation transport, and therefore dose distribution, resulting from the presence of beam-shaping devices. The convolution method used here has demonstrated improved accuracy comparing to a scatter integrating- approach and at the same time requires smaller amounts of computer time. The limitation of this method is in the objective to make dose constant for an individual beam and in a specified plane.

Optimal solution of compensator design for multiple beams requires an optimization approach. Redpath et al. (1976) optimizes only beam weights and makes no provision for designing compensators for each beam. Renner et al. (1989) proposed an algorithm based on linear programming approach. The method designs an optimal compensator and calculates beam weights for optimal dose in the target region. The dose to other vulnerable regions is not considered. Djordjevich et al. (1989) has developed an optimization technique for design of variable-thickness filters in multibeam situations. Shapes, size and weights of the beam are determined by the technique. The constraints applied are dose limits to vulnerable points and minimal dose to the healthy tissue. The deviation of the dose delivered from the prescribed dose to a tumor should be minimized as well. The filters provide dose to target zone as close as possible to the prescribed one, dose to designated vulnerable regions lower than maximal 
tolerable at each region and minimal overall dose to the healthy region under consideration. Radiation quality and directions of beams have to be predetermined. The basic idea is to divide incident radiation beams into a number of "elementary" beams. It is assumed that the dose deposited to a point of interest is a linear function of the beam intensity. The result of an optimization process is the desired intensities elementary beams that indicate how much each elementary beam should be pre-attenuated relative to an adopted "reference-intensity" beam, thus specifying the needed filter thickness.

The Gauss-Marquardt nonlinear least-squares regression technique was applied to compensator design by Weeks and Sontag (1990). The dose distribution (uniform or otherwise) desired throughout a volume is specified. Compensators optimized to produce the necessary variation of beam intensity across the surface of each beam are simultaneously determined for all beams. The technique is relatively time consuming for fine compensator resolutions.

A simple formula was derived to calculate the optimal compensator thickness profile from an optimized primary fluence profile by Jiang and Ayyangar (1998). It was found that compensator significantly changes the energy spectrum and the mean energy of the primary photons at the patient's surface. However, beam hardening does not have as significant an effect on the Percentage Depth Dose (PDD) as it does on energy spectrum. The influence on the compensator on the contaminant electron buildup dose is found to be small and independent on the compensator thickness of interest. Therefore, it can be ignored in the compensator design and included as a correction into the final dose distribution. The scattered photons from the compensator are found to have no effect on the surface dose. Compared to the primary photons, the scattered photons have much larger angular spread and similar energy spectrum at the patient's surface. These characteristics allow the compensator thickness profile and the dose distribution to be calculated from the optimized fluence profile of primary photons, without considering from compensator scattered photons. A primary transmission function for a parallel photon beam is precalculated for various thickness of compensator material for a given energy spectrum. The data are stored in a look-up table and are available for interpolation. During optimization process the beam is divided into $\mathrm{n}$ rays. Initially each ray is assigned a unit fluence, then at the end of the process a weight is obtained for the ith ray and hence a relative primary fluence at the patient's surface.

Accurate production of compensators with complex surface profiles is best achieved by computer numerical control (CNC) milling machines. The compensator shape can be milled 
directly in compensator material or a complementary image of the compensator shape can be milled into a mold, which then can be filled with compensator material. The quality limitations are due to the finite size of the cutter. The major drawback of the use of compensators is the amount of time required to manufacture each individual beam modifier, especially a milling tool of small diameter and small distance about adjacent cuts (step-over distance) is used (Meyer et al. 2000). If the tool diameter is increased, coarser surface is produced, thus the lower resolution of the intensity profiles is achieved. The effect of the surface unevenness is more significant of a high density material.

The resolution in the longitudinal direction (see Fig. 2-1(a)), along the path of the cutter, depends on the size of the cutting-tool diameter and the angle of the profile. In the lateral direction- from the size of the cutting-tool diameter, angle of the profile and step-over distance as well. The desired profile can be described by means of flat, oblique, with a given angle $\alpha$ and "V" shaped (valley), with given angle $\beta$, surfaces, as illustrated by Figs. 2-1(b) and 2-1(c) for longitudinal and lateral directions. Influence of the tool diameter on the overall machining time was found to be insignificant; whereas the machining time decreases significantly if step-over distance is increased. The maximum allowable absolute fractional error in the intensity profile restricts maximal angles possible to cut with the cutter of given diameter and given step-over distance. Increasing the radius of the cutter for a fixed step-over distance allows larger angles to be machined for oblique slopes in the lateral direction. However, the angles for the "V" shape features in lateral and longitudinal directions become shallower as both the step-over and the total cutter radius increases. In addition, the maximum angle for the "V" shape feature is larger in the longitudinal than in the lateral direction. So, the direction of the machining process should be oriented such that the steepest slopes are in the longitudinal direction.

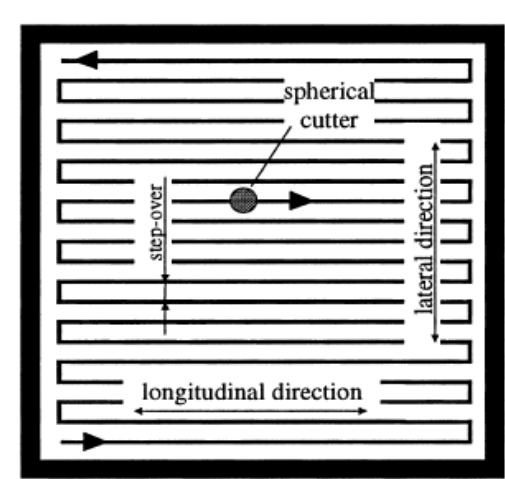

(a)

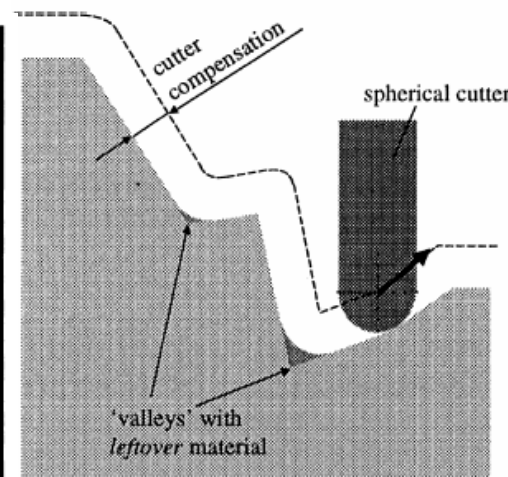

(b)

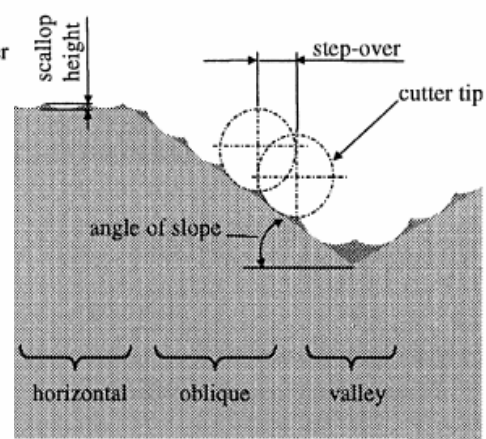

(c)

FIGURE 2-1. Cutter movement directions for milling a compensator (a) and compensator profiles in longitudinal direction (b) and in the lateral direction (c) (Meyer et al. 2000). 


\subsection{TOMOTHERAPY}

In the late 1980s, the University of Wisconsin (UW) Radiotherapy Research Group began to explore methodologies for intensity modulation of radiotherapy beams. It was concluded that the simplest method would be to move rapidly a bank of temporally modulated collimators into and out of a narrow fan beam of radiation. It was reasoned that the dose could be delivered slice-by-slice in the fashion of an axial computed tomography (CT) scanner. The idea was called tomotherapy that literally means "slice therapy" (Mackie et al. 1999). Discouraged by a belief that this sequential translate-then-rotate, slice-by-slice treatment technique would produce discontinuities at the junction between slices, however, the concept was shelved. Subsequently, MEDCO Corporation (which later became NOMOS, Sewickley, PA) concluded that IMRT with a temporally modulated fan beam was the most practical approach even if used on a conventional linear accelerator (linac) incapable of helical beam delivery and with limited range of rotation. NOMOS corporation has developed the Peacock $^{\mathrm{TM}}$ three-Dimensional Conformal System (Carol 1995), which plans for and implements, through the use of a multileaf intensity modulating collimator $(\mathrm{MIMiC})^{\mathrm{TM}}$, conformal treatment plans in a slice-by-slice fashion. The MIMiC is an electropneumatic implementation device, which slides into the wedge/blocking/accessory tray slot on the treatment machine. Its schematic illustration is shown in Fig. 2-2. The MIMiC functionally

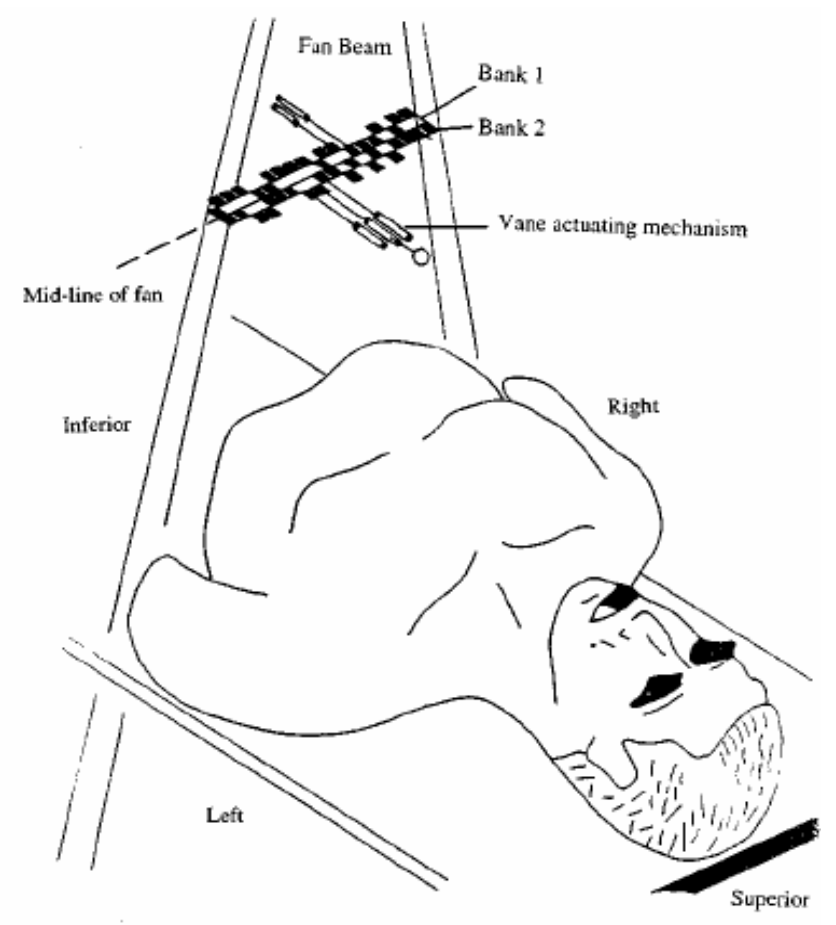

FIGURE 2-2. A schematic illustration of the slit beam of the MIMiC and its bixels in relation to a patient. Open bixels are shown as white spaces and closed bixels as black spaces (Oldham and Webb 1997). 
narrows the beam coming from the accelerator down into thin "slices", then further divides these slices into 40 smatter beams, 20 for each slice. Each of these "pencil" beams can be turned on and off by driving an 8-cm-tall divergent tungsten block into its path. It takes 40-60 milliseconds for valve movement in or out of the field. Because this modulation is delivered in a slice-by-slice fashion, a complete treatment is accomplished by stacking a series of slices.

The rotation around the patient can only treat the equivalent of two slices through the patient (see Fig. 2-3); the table has to be successively indexed forward to treat the rest of the target. To minimize the magnitude of homogeneity caused by adjacent fields mismatch, precise and predictable indexing of the treatment coach is important. A special table indexing device (the Crane) ${ }^{\mathrm{TM}}$ was therefore developed for use with Peacock. The setup of the patient is assured using an invasive immobilization system called the Talon ${ }^{\mathrm{TM}}$ that is screwed into the patient's skull and docks rigidly to the imaging system and treatment couch.

The MIMiC can be used either in rotational mode, where the vanes move as gantry rotates, or static mode, where the vanes move but the gantry is fixed at some orientation. The former is conventionally termed tomotherapy and the latter is referred as static tomotherapy (Oldham and Webb 1997). A treatment is delivered in rotational fashion functionally viewed as a series of fixed ports; every $5^{\circ}$ of rotation is treated as a separate port. As a gantry rotates around the patient with the accelerator turned on, turning individual "pencil" beam on or off
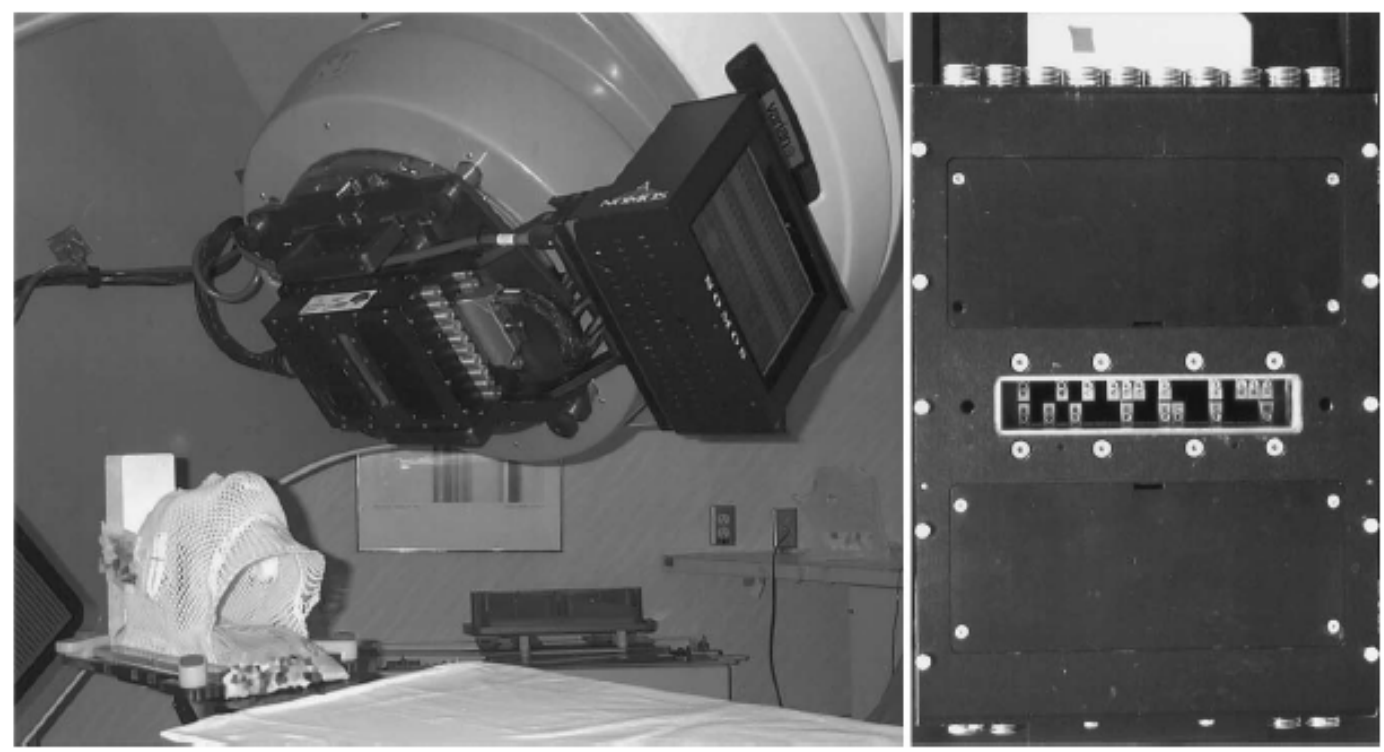

FIGURE 2-3. Serial tomotherapy approach to IMRT. This form of IMRT uses a temporally modulated MLC system such as MIMiC (NOMOS Corp.) shown here mounted to a conventional low-energy megavoltage medical linear accelerator. Treatment to a narrow slice of the patient is delivered by arc rotation. A complete treatment is accomplished by serial delivery to adjoining axial slices. (IMRT-CWG 2001). 
for a variable period of time during the $5^{\circ}$ arc controls the effective attenuation of the beam during that $5^{\circ}$ of arc treated as if it were a fixed port. In 1994, the Peacock ${ }^{\mathrm{TM}}$ system was the first form of collimator-based IMRT to treat a patient.

On the introduction of helical CT scanners, tomotherapy was reexamined, and it was reasoned that a fan beam continuously rotating about a patient would eliminate beamjunctioning artifacts. A spiral pattern would tend to eliminate these junctions because the helical fan beam would provide blurring in the longitudinal direction. The main idea of tomotherapy is to put a linear accelerator into a CT-like ring gantry configuration and deliver therapeutic radiation using a rotation fan beam slit, which is modulated by a multileaved collimator system, while a patient moves throughout the gantry in the longitudinal direction (Webb 2001). Such system incorporates both a tomographic imaging system for treatment verification and tomographic reconstruction mathematic for optimal treatment planning, as shown in Fig. 2-4.

\section{Tomotherapy Unit}

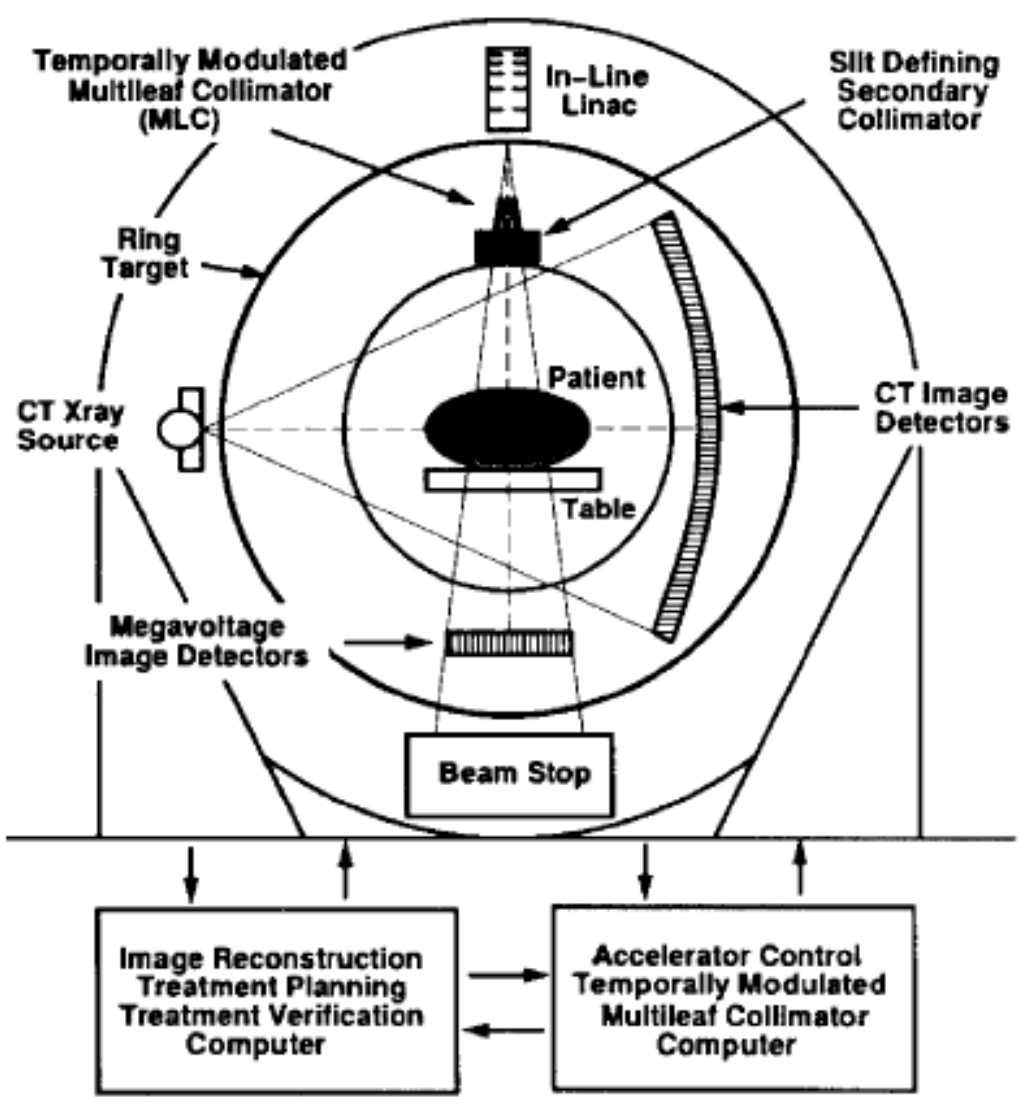

FIGURE 2-4. Conceptual drawing of a helical tomotherapy unit in the first tomotherapy paper (Mackie at al. 1993). 
The temporally modulated multileaf collimator system is a key to tomotherapy design. It consists of as set of singly focused, tall, narrow leaves. A pair of jaws defines the width of the fan beam. Leaves are in the close state until retracted to the open state to allow radiation to be emitted. The field intensity through an open leaf during a short segment of arc is proportional to the amount of time that the collimator leaf is open.

The collimator leaves are temporary modulated using electromagnetic actuators or pneumatic pistons. The number of possible intensity levels would be constrained by the speed at which the collimators can be switched and the repetition rate of linac. A potential limitation with a tomotherapy unit is the inability to deliver from all non-coplanar directions.

Tomotherapy's greatest advantage may ultimately be its potential to make superior treatments routine and less expensive by requiring less technical expertise or unusual time investments per patients. A tomotherapy unit would reduce the possible treatment geometry degrees-of-freedom, somewhat, in order to safely and routinely perform dynamic conformal radiotherapy, while providing unprecedented dynamic verification of dosimetry and treatment geometry.

Tomotherapy research has become more clinically focused in recent years. The fabrication of the UW unit was completed in early 2001 and a long software integration and validation process followed. On 21 August 2002, JimWelsh, at the UW treated the first human patient with helical tomotherapy system. In 2005 almost 500 protocol patients have been enrolled with enormous progress in lung, prostate and head and neck cancers (Mackie 2006).

The development of new systems incorporates the common theme of image guided therapy (IGT). The helical tomotherapy machine has an integrated Megavolt Computed Tomography (MVCT) imaging capability. A conventional xenon ion chamber CT detector system is mounted on the gantry directly opposite to the beam-line. This offers two benefits for IGT: the obvious one being the acquisition of volumetric CT imaging of the patient at the time of treatment delivery. Secondly, during treatment the intensity of the photons that exit the patient can be collected and used in a back-projection computation to assess the dose distribution that is delivered to the patient. The helical tomotherapy is not just a dedicated IMRT delivery system but as an IGT delivery system (Beavis 2004). 


\subsection{IMRT WITH MLC}

In order to carry out field shaping for conformal radiotherapy, MLCs are being developed and implemented by the major manufacturers of medical linear accelerators. MLC consists of a large number of highly absorbing tungsten leaves (about 20-80 on each side of the treatment field) that can be moved against each other and thereby create a large variety of field openings (see Fig. 2-5), which can be shaped to conform the projection of the tumor target volume (Bortfeld 2006). IMRT can be delivered with a conventional multileaf collimator, either in dynamic mode (DMLC) or in segmental mode (SMLC, also known as ' "step-and-shoot') ). In the SMLC method, each intensity-modulated (IM) field is delivered in a sequence of "step"s, in which the leaves move to their respective designated positions while the beam is off, and "shoot"s, in which the leaves remain stationary while the beam is turned on. In the DMLC method, the leaves move continuously at variable speeds while the beam is on for a fixed gantry position.

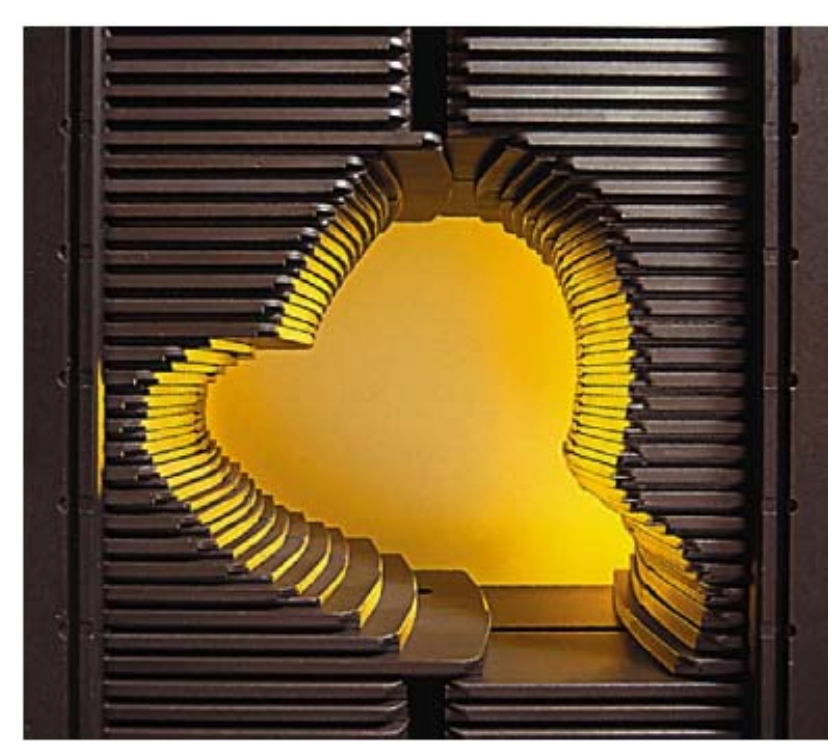

FIGURE 2-5. A multileaf collimator consists of a large number of highly absorbing tungsten leaves ( in the order of 20-80 on each side) that can be positioned individually to create field openings with complex shapes. The beam orientation is perpendicular to this shape (Photo of Varian Medical Systems).

Takahashi (1965) has studied the use of the MLC for the rotational conformal therapy. The concept of in-field intensity modulation through the motion of an MLC was first proposed by Brahme (1988). Källman et al. (1988) developed an algorithm to compute the irradiation density required to produce an arbitrary dose distribution. Convery and Rosenbloom (1992) have introduced an algorithm that calculates the MLC leaf motions 
necessary to produce an intensity-modulated field by dynamic collimation. Improvements to the Convery and Rosenbloom approach were developed independently and almost simultaneously Svensson et al. (1994), Spirou and Chui (1994) and Stein et al. (1994). Bortfeld et al. (1994) introduced an algorithm that computes the MLC leaf movements necessary to produce an arbitrary distribution of dose for SMLC mode. The MLC leaves are moved through the delivery sequence in a series of discrete steps. This algorithm had an objective to minimize the number of MUs delivered that primarily affects the undesired leakage of radiation. Independently, Galvin et al. (1993) developed a step-and shoot intensity modulation technique whose objective was to reduce the total number of segments, which on some treatment machines governs the treatment time. Their approach was to 'ablate' the intensity map from top to bottom using segments with ever decreasing MU settings (Galvin et al. 1993). This technique has later been refined and called 'reducing level technique' (Xia and Verhey 1998).

The advantage of DMLC is its ability to deliver the desired intensity profile produced by inverse planning with a high degree of fidelity, accurately preserving both the spatial and intensity resolutions. The SMLC method, on the other hand, resembles treatment with multiple static fields, and therefore can be more easily verified (Chui et al. 2001). The latter is also less demanding in terms of quality assurance, for only the leaf positions needs to be checked, whereas in DMLC both the leaf position and the speed need to be checked. However, the use of SMLC requires the desired profile to be approximated by discrete levels of intensity. Inevitably, the conversion from the original profile to the discrete one leads to some degradation in the dose distribution. Clearly, the degree of degradation of the SMLC method depends on the spatial resolution and the number of intensity levels used in the approximation. In the study of Keller-Reichenbecher et al. (1999) it was suggested that the results from a 5-level SMLC delivery were comparable to that from the DMLC delivery. A spatial resolution used in that study as $5.5 \mathrm{~mm}$. It was concluded that the number of levels mostly affects the target coverage for SMLC plans, while the spatial resolution has more significant effects on critical organ sparing. For example, for a nasopharynx case, when the spatial resolution was changed from $2 \mathrm{~mm}$ to $10 \mathrm{~mm}$, target coverage was modestly affected, but critical organ sparing was deteriorated significantly. It was observed that both methods give comparable results provided sufficient number of levels were used. 10 levels usually give comparable results relative to the DMLC method. There is a little to be gained by increasing to more than 20 levels, and in some cases it is adequate to use only 5 levels. In general, the 
beam-on-time (in MUs) required for the DMLC delivery was about 20\% more than the SMLC delivery. However, the actual delivery time (in minutes) for SMLC was about 2 to 2.5 times of that for DMLC. For SMLC delivery, the beam-on-time (MUs) does not appear to be much dependent on the number of levels.

Today treatment times in order of $10 \mathrm{~min}$ for an entire IMRT treatment are achievable. Both dynamic and step-and-shoot delivery are in clinical use. Further progress has also been made on the algorithmic side of leaf sequencing for both techniques (Bortfeld 2006).

In some recent inverse planning algorithms the optimization of the intensity maps and the subsequent leaf sequencing step have been abandoned altogether and the MLC segment shapes (apertures) are directly optimized (De Gersem et al. 2001). This approach has been called direct aperture optimization (Shepard et al. 2002) or direct machine parameter optimization. The removal of the intensity map optimization as an intermediate step in inverse planning makes the problem much more difficult (non-convex) from a mathematical point of view. Direct aperture optimization algorithms are not guaranteed to find an optimal IMRT solution. In spite of this mathematical difficulty, practical direct aperture optimization algorithms based on simulated annealing have generated excellent IMRT plans with a small number of segments, especially when fed with a good initial guess of the segment shapes (Bortfeld 2006).

Both MLC-based intensity modulation delivery methods (static and dynamic) are subject to mechanical restrictions due to the finite width of their leaves (Meyer et al. 2000).

Longitudinal direction: For static delivery, the resulting profile is a "staircase" approximation, whilst by dynamic delivery it is a linear approximation. The leaf positions theoretically do not have to be equally spaced and could be adjusted to high spatial frequencies in the appropriate parts of the profile. By increasing the number of leaf positions, the resolution increases.

Lateral direction: In lateral direction the resolution is characterized by leaf widths and is independent on the delivery method. The root-mean-square error between intended and delivered intensity profile decreases in an exponential manner with the inverse of leaf width. More highly modulated profiles can only be produced satisfactorily with the $0.1 \mathrm{~cm}$ and 0.3 cm MLC. This drawback might be overcome by including the limitations of the MLC when generating the Intensity Modulated Beams (IMBs). Another suggested method would be to swap the longitudinal/lateral direction of the leaves halfway through the treatment. During the 
planning there is a need to consider carefully the orientation of the beam delivery technique relative to the modulation in the intensity profile.

MLC-shaped fields, which change shape during gantry rotation, are used also in IMAT technique. Intensity-modulated arc therapy (IMAT) is a technique that delivers optimized dose distributions by rotating the radiation beam around the patient ( $\mathrm{Yu}$ 1995). It combines spatial and temporal intensity modulation with movement of the gantry. Arbitrary intensity distribution at each angle is delivered with multiple arcs. A radiation arc treatment is approximated as many fixed fields at small angular intervals of gantry rotation. At each beam angle, the two-dimensional intensity distribution generated by the inverse treatment planning techniques is considered as superposition of multiple radiation fields of different sizes and shapes each with uniform intensity. Since each of these subfields must have a single contiguous opening, complex intensity distributions with multiple fields and valleys or with islands fields generally require additional superimposing arcs. It can be shown that the dose conformity is theoretically equivalent to that achievable with slice based treatment techniques. Patient set-up time is the same as conventional treatments. Beam delivery time is proportional to the number of arcs required, which depends on the number of intensity levels and the complexity of the intensity distributions. The technique can be easily adapted to any linear accelerator equipped with a MLC (see Fig. 2-6).

A new technique for IMBs delivery that combines the features of the original IMAT technique with those of classical blocks was proposed by Cotrutz et al. (2000). The role of the blocks is to shield radiosensitive regions within the patient. In other words, the steep gradient

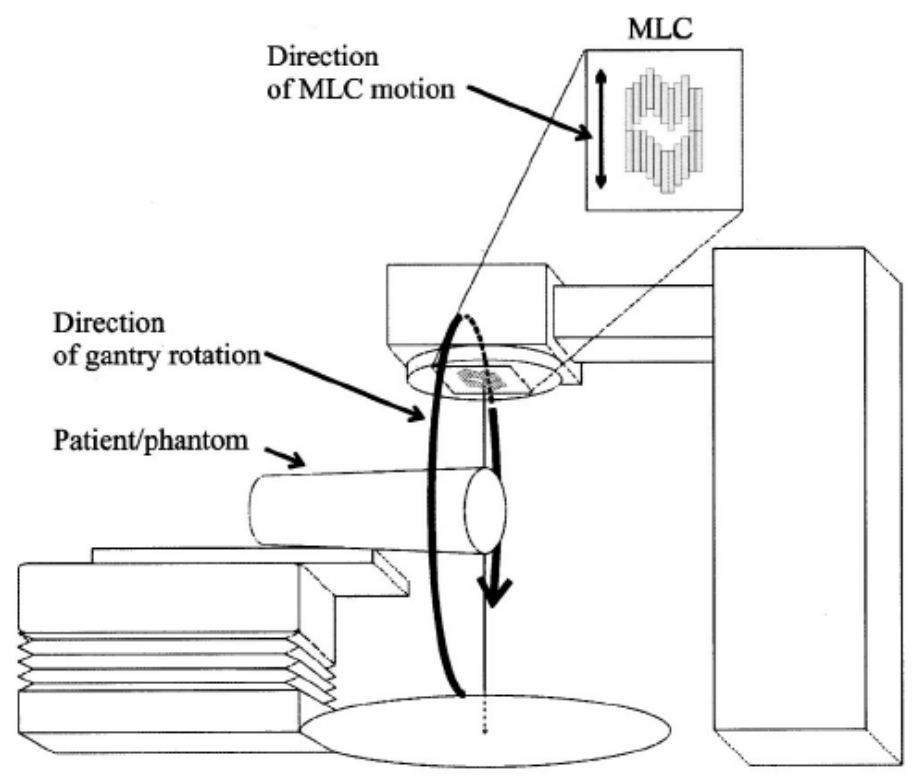

FIGURE 2-6. Experimental setup for IMAT delivery (MacKenzie and Robinson 2002). 
intensity modulation of the fluence profiles is realized with the aid of the blocks, while the MLC leaves or the secondary collimator defines the rest of the field and delivers the necessary intensity modulated field to achieve as homogeneous a dose as possible to the target. The linear optimization routine of precalculated dose profiles chooses the appropriate field sizes and weights of the segments, which in combination will deliver a constant dose level to the PTV and dose gradient between the OAR and PTV doses.

In comparison with the sliced delivery schemes, IMAT has many advantages ( $\mathrm{Yu}$ 1995). It is implemented on existing linear accelerator equipped with an MLC. Therefore it maintains the flexibility of a linear accelerator. Non-transaxial arc treatments can be achieved to a certain extent and partial arc rotations are easily achievable. Since it does not collimate the beam into a slit, most of the target will be in the beam during the delivery, maintaining a high efficiency in utilizing the photons generated in the x-ray target. No additional patient transport mechanisms are required to move the patient from slice to slice. Eliminating the slicing also eliminates the problem of beam abutment between slices, and the cold and hot spots associated with the abutments. Finally, since the intensity modulation in tomotherapy relies on a set of valves to open or close the slit beam, the resolution of the beam intensities is the slit width by the leaf width. For IMAT treatments, the resolution is the width of the leaf in the leaf width direction and continuous in the length direction of the leaves.

For IMAT to be practical, the number of intensity levels at each gantry angle has to be limited. This limitation is imposed by treatment time considerations and by lowest number of MUs per degree of gantry rotation allowed by the linear accelerator. No more than five intensity levels are needed for IMAT treatments.

Alternative method is to use a single arc for the treatment. The technique proposed by MacKenzie and Robinson (2002) uses sliding window DMLC to deliver IMRT ports, instead of variable MLC apertures, to produce highly conformal dose distributions. These conventional IMRT fields are delivered at a large number (24) of equally spaced static gantry positions (every 15 degrees) over a single full gantry rotation, as opposed to a large number of gantry passes with continuous beam delivery. Crooks et al. (2003) tried to reduce the number of segments per irradiation direction and combined them in single rotation too. They called their method aperture modulated arc therapy (AMAT). Direct aperture optimization algorithms (De Gersem et al. 2001, Shepard et al. 2002) are used as a means of generating AMAT plans. 


\subsection{ALTERNATIVE IMRT METHODS}

Some other trials to perform an intensity modulated treatment delivery leaded to a development of such techniques as use of swept pencils of radiation (Brahme 1987), intensity modulation by means of single computer-controlled absorber (Fiorino et al 1995), reshapable physical modulator (Xu et al. 2002), packed building-block compensator (TETRIS-RT) (Nakagawa, Fukuhara and Kawakami 2005), IMRT delivery by robotic linac (Webb 1999, Webb 2000), multiportal compensation system (Yoda and Aoki 2003) and multiple isocenter IMRT using circular collimators (Galvin 2006).

Another alternative kind of IMRT is the rotational radiotherapy with gravity-oriented devices, first developed and reported in the 60's (Proimos 1961, Proimos 1966, Proimos et al. 1966). Let us extend the description of the above technique, because of the close relation of the beam modifying devices, used in intensity modulation studies in the work set forth in the chapters 4 and 5, with the Proimos' gravity-oriented devices.

The idea of synchronous protection of the OARs in the rotational therapy was originated by Basil Proimos in 1957 (Danciu 2001). The patient was rotated about a vertical axis passing through the center of the involved region. The stationary horizontal beam was carefully shaped by external absorbers. This concept was also applied to the spinal cord protection and involved the synchronous rotation of the patient and the protector that reminds in shape the spinal cord around two respective vertical axes (Wright et al. 1959, Proimos 1960). This idea was further developed in early 60's for horizontal and immobile patient and the source, rotating around the patient in a vertical plane (Proimos 1961). In such a case, the protectors are gravity-oriented. The protectors used are diminished copies of the Organs At Risk and stay parallel to them during gantry rotation. In other words, the protectors always remain "omoiotheta" to the corresponding OARs. "Omoiotheta" is a compound word of the Greek words "ó $\mu \sigma_{0} \varsigma$ " that means "same, similar" and " $\theta \dot{\varepsilon} \sigma \eta "$ that means "position". Thus, each protector always keeps the corresponding OAR in its shadow, significantly reducing the irradiation. Moreover, the beam sides can be shaped by means of gravity-oriented absorbers to conform the beam's cross-section to the beam's eye view of the PTV for every gantry angle and, thus, to reduce the dose to the adjacent healthy tissue (Proimos 1960, Proimos 1961, Proimos 1963, Proimos 1966, Proimos 1969).

During three decades from 60's to 80's gravity-oriented devices were designed, built and used for a variety of clinical applications, such as for the protection of the spinal cord 
during a treatment of the cancer of larynx (Proimos 1961) and esophagus (Proimos 1963, Proimos 1966, Proimos and Goldson 1981); protection of the bladder and rectum in the case of the cervix cancer (Proimos 1963, Proimos 1966, Proimos1969); for a uniform dose in arc therapy of eccentric tumors (bladder, larynx, antrum, etc.) (Proimos et al. 1966); and for protection of the eyes in facial tumors (Engler et al. 1984).

Recently further ideas regarding gravity-oriented devices were published (Proimos and Danciu 1997a, Proimos and Danciu 1997b, Danciu and Proimos 1999) and the technique has been further developed, simplified and standardized (Danciu 2001).

\subsection{CONCLUSIONS: FOR AND AGAINST IMRT}

IMRT techniques show great potential in terms of achieving very high degree of target conformity and healthy tissue sparing, particularly for target volumes and organs at risk (OAR) with complex shapes (IMRT-CWG 2001, Pirzkall et al. 2000). Synergetic development of

- $\quad$ fast computing

- $\quad$ radiation delivery technology

- computer control

- inverse planning theory and practice

- 3D imaging and planning

- dissatisfaction with static nature of radiation therapy practice

leaded to rapid development of IMRT. IMRT has the potential to reduce the complications that follow the conventional external beam irradiation (Hunt et al. 2001), so this could be important in terms of quality-of-life (Jabbari et al. 2005). However, the complexity and the cost of implementing and maintaining these new technologies are the major justifications for their limited clinical application. Moreover, there are not clinical proofs that a treatment with these expensive new technologies increases the patients' survival. Inevitably there remain fierce skeptics of its need, practicability and safety (Schulz 1999, Schulz 2001, Schulz and Kagan 2002). But there is a clinical evidence that technologies which allow increased dose to the tumor while sparing healthy tissues will improve the balance between complications and cure (Keall and Williamson 2003). 
The obvious benefit of the MLC-based IMRT techniques is treatment delivery automation that saves radiation therapist trips into the treatment room. The use of MLC does not involve manufacturing of physical object. MLC-based IMRT techniques, in its turn, have shown several drawbacks in clinical application, such as:

1. increased technical and mechanical complexity that weakens the benefits gained by automation. In study of Miles et al. (2005) it was shown that physics time increase 4.9 $\mathrm{h}$ per patient after IMRT treatment introduction in the clinic.

2. total monitor units (MU) required for segmental MLC-IMRT treatment are often much higher than for the corresponding nonintensity modulated treatment, therefore the treatment delivery time is often considerably extended;

3. intensity modulation is discrete at least in one direction;

4. complicated quality assurance procedure.

A 3D-CRT with physical modulators offers lower cost, simpler quality assurance, higher resolution in the direction normal to the leaf motion and shorter delivery time of the treatment that results in less irradiation of the whole body due to the head leakage (Chang et al. 2004). On the other hand it has relatively cumbersome and time-consuming manufacturing process as well as one must enter the treatment room to change the filter for each gantry position (IMRT-CWG 2001).

Sherouse (2002) does not agree with the assertion of the IMRT-CWG (2001) that the use of physical filters to deliver fluence pattern "will likely serve only as an intermediate step in some institutions before other approaches to IMRT are adopted". Sherouse defines solid filters as gold standard, and MLC as acceptable compromise. Reliability of hard filter is better, as it do not ever fail to absorb photons. Physical modulator offers time invariance, with together with patient motions and produce significantly smaller error, than when beam fluence patter is also time dependent. The money and time cost of implementation, validation and routine use are significantly lower, and quality of the product by any objective criterion is higher than with MLC.

The evident advantages of the compensator-IMRT technique are (Chang et al. 2004):

1. simplicity of treatment delivery, dose computation, and thus the quality assurance;

2. continuously varying fine intensity modulation;

3. shorter treatment delivery time, comparing with MLC-IMRT technique (less than 5 min versus more than 10 min for a typical five-field prostate treatment); 
4. good results for evaluation of a deviations between measured and calculated intensity profiles and dosimetry.

The drawbacks of the technique:

1. lack of automation, but this is well compensated by the delivery efficiency of the compensator technique;

2. fabrication and assembly time (fabrication and QA procedure is $13 \mathrm{~min}$; disassembly and recycling is $2 \mathrm{~min}$ );

3. density of compensator material and its maximum thickness limit the intensity modulation range.

Arguments for and against IMRT (Webb 2001):

1. Considered potentially dangerous since it "too tightly" conforms the high dose distributions, opposite it allows greater tolerance of location errors since IMRT inherently spares more of the adjacent normal tissue.

2. Development of sophisticated technology has an unstoppable momentum largely because the electrotechnical companies are making huge investments at the same time there is climate of cost- containment in hospitals. Discussion to implement or not to implement may not be influenced by scientific arguments only.

IMRT is not a treatment philosophy in itself but a delivery technology. Attempts to define IMRT as an advanced form of 3D conformal radiotherapy are misleading as IMRT can be used to deliver a broad range of dose distributions that may not necessary involve high conformality (Bentzen 2005). The hallmark of IMRT is the use of non uniform radiation beam intensities incident on the patient to shape the dose distribution in the target volume and in the adjacent normal structures. IMRT is not a magic bullet by itself; if strictly the same target selection and delineation is performed, and the same biologically effective dose is delivered to the tumor there is no inherent reason why tumor control after IMRT would be superior to conventional delivery. However, IMRT can deliver 3D conformal radiotherapy plans, generally with the closer match between the clinical target volume and the high dose region of the delivered dose map than what, which is achievable using conventional delivery methods, so can be used to intensify treatment and reduce treatment toxicity. 


\section{INVERSE PLANNING PROBLEM IN RADIATION THERAPY}

\subsection{INTRODUCTION}

None of the uniform beam irradiation techniques can produce a strongly concave treatment volume except to some extent for the fairly complex multicenter arc treatments (Brahme 1988). With non-uniform incident beams, the flexibility is much greater and even a two or three field technique with the beam properly weighted can do the job. The 1982's paper by Brahme, Roos, and Lax (BRL) was the first which posed the central question of the inverse planning problem in IMRT: "Which is the desired lateral dose [intensity] profile in the incident beam that produces a desired absorbed dose distribution in the body after one complete rotation?" Actually, now it is recognized as the definition of the inverse planning problem in IMRT (Bortfeld 2006).

\subsection{DIRECT INVERSE PROBLEM}

The first exact answer to the inverse problem was given for a special idealized case of a donut shaped dose distribution received with rotational geometry with blocking of the central volume. The assumption of the exponential photon absorption, neglect of lateral scattering 
effects and parallel beam geometry was used (BRL approximation). It was shown that the radiation field required to produce the dose distribution could be calculated by solving a certain integral equation.

\subsubsection{Dose distribution of radial symmetry}

\subsubsection{Mathematical formulation of the problem}

The dose distribution in the cylinder depicted in Fig. 3-1 can be written:

$$
\mathrm{d}(\mathrm{x}, \mathrm{z})=\mathrm{d}(\mathrm{x}) \exp (-\mu \mathrm{z})
$$

where $\mathrm{d}(\mathrm{x})$ is the beam intensity variation along the positive $\mathrm{x}$ axis and $\mathrm{z}$ is the distance from the $\mathrm{x}$ axis in the direction of the beam, if we consider the dose is proportional to the intensity, and $\mu$ is linear attenuation coefficient of the phantom.

When a beam is rotated one complete turn, all points at a radial distance $r$ in the cylinder, will receive an absorbed dose equal to the line integral over the absorbed dose distribution along a circle of radius $r$ in the cylinder:

$$
\mathrm{D}(\mathrm{r})=\oint_{\mathrm{r}} \mathrm{d}(\mathrm{x}) \exp (-\mu \mathrm{z}) \mathrm{d} \varphi / \pi
$$

By using the polar coordinate transforms and for case of rotation symmetry, the resultant radial dose variation becomes (Brahme et al. 1982):

$$
\mathrm{D}(\mathrm{r})=\int_{0}^{\mathrm{r}} \frac{\mathrm{d}(\mathrm{x}) 2 \cosh \mu\left[\left(\mathrm{r}^{2}-\mathrm{x}^{2}\right)^{1 / 2}\right]}{\pi\left(\mathrm{r}^{2}-\mathrm{x}^{2}\right)^{1 / 2}} \mathrm{dx} .
$$

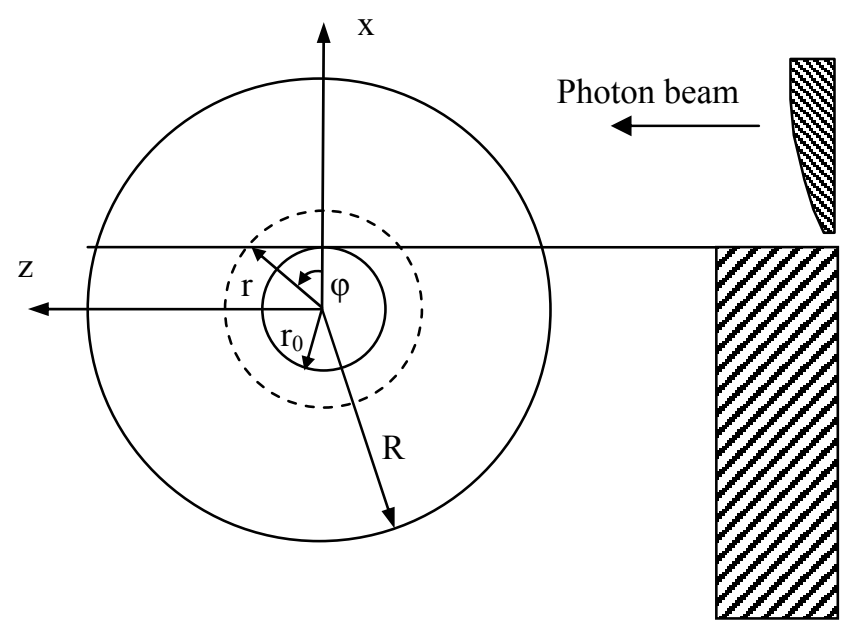

FIGURE 3-1. The irradiation geometry and coordinate system used in the calculations. The location of the beam block, shielding the central part of the phantom of radius $\mathrm{r}_{0}$, and non-linear filter are indicated. 
The lateral intensity distribution in the incident beam $d(x)$ should thus satisfy the above integral equation, where $\mathrm{D}(\mathrm{r})$ is the desired dose distribution using rotational therapy.

\subsubsection{General analytical solution}

The integral equation 3-3 has to be solved and the function $\mathrm{d}(\mathrm{x})$ has to be determined in terms of $\mathrm{D}(\mathrm{r})$. The solution will be derived for the case, in which $\mathrm{d}(\mathrm{x})$ is zero inside a circular region of radius $r_{0}$. Here, $0<r_{0} \leq r \leq \infty$ and $\mu>0$ is a small constant, $D(r)$ is a given function, which is zero for $0 \leq \mathrm{r}<\mathrm{r}_{0}$.

(a) If $\mu$ is very small (for high photon energies) and $r$ is close to $r_{0}$,

the approximated Eq. 3-3 in the form of the well-know equation of Abel:

$$
\mathrm{D}(\mathrm{r})=\left(\frac{\mathrm{r}_{0}}{2}\right)^{1 / 2} \frac{\mathrm{d}}{\mathrm{dx}} \int_{\mathrm{r}_{0}}^{\mathrm{x}} \frac{\mathrm{D}(\mathrm{r})}{(\mathrm{x}-\mathrm{r})^{1 / 2}} \mathrm{dr}
$$

and the solution is as following:

$$
\mathrm{d}(\mathrm{x})=\left(\frac{\mathrm{r}_{0}}{2}\right)^{1 / 2} \frac{\mathrm{d}}{\mathrm{dx}} \int_{\mathrm{r}_{0}}^{\mathrm{x}} \frac{\mathrm{D}(\mathrm{r})}{\left(\mathrm{x}-\mathrm{r}_{0}\right)^{1 / 2}} \mathrm{dr} .
$$

In particular, if $D(r)$ is constant $=\mathrm{D}$ for $\mathrm{r} \geq \mathrm{r}_{0}$, it follows that

$$
\mathrm{d}(\mathrm{x})=\frac{\mathrm{D} \cdot\left(\mathrm{r}_{0} / 2\right)^{1 / 2}}{\left(\mathrm{x}-\mathrm{r}_{0}\right)^{1 / 2}}
$$

Figure 3-2 illustrates the required intensity distribution necessary to achieve the desired constant dose $D(r)=D$ for $r \geq r_{0}$ and $D(r)=0$ for $r<r_{0}$. It was assumed $r_{0}$ to be $4 \mathrm{~cm}$, while phantom radius $\mathrm{R}$ to be $6 \mathrm{~cm}$. The dose value of $\mathrm{D}$ was arbitrary set to 1 . As it can be noted the solution of the integral equation (Eq. 3-3) has a square root singularity.

(b) Explicit solution, i.e., if $\mu \neq 0$ and $\mathrm{r}$ is not necessarily close to $\mathrm{r}_{0}$.

Equation 3-3 is a generalized Abel integral equation, which can be solved by using Laplace transforms

- if $D$ is constant

$$
d(x)=D \frac{x}{\left(x^{2}-r_{0}^{2}\right)^{1 / 2}} \cos \left[\mu\left(x^{2}-r_{0}^{2}\right)^{1 / 2}\right]
$$




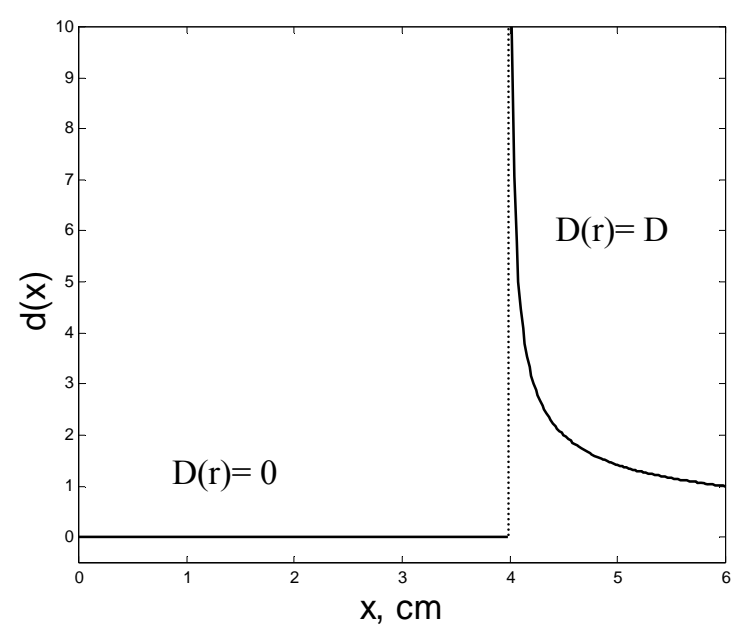

FIGURE 3-2. The lateral intensity profile across the radius of the cylindrical phantom derived using simplifications of small $\mu$, and that $r$ is close to $r_{0}$. The values of $r_{0}$ and $R$ were $4 \mathrm{~cm}$ and 6 $\mathrm{cm}$, correspondingly. The dose $\mathrm{D}$ was arbitrary set to 1 .

for $\mathrm{x} \geq \mathrm{r}_{0}$ and $\mathrm{d}(\mathrm{x})=0$ for $\mathrm{x}<\mathrm{r}_{0}$. The lateral intensity profile for this case is depicted in Fig. 33 , with $\mathrm{r}_{0}=4 \mathrm{~cm}, \mathrm{R}=10 \mathrm{~cm}$ and linear attenuation coefficient $\mu=0.05 \mathrm{~cm}^{-1}$.

- if $D$ is not constant

$$
\mathrm{d}(\mathrm{x})=\frac{\mathrm{d}}{\mathrm{dx}} \int_{\mathrm{r}_{0}}^{\mathrm{x}} \frac{\cos \left[\mu\left(\mathrm{x}^{2}-\mathrm{r}^{2}\right)^{1 / 2}\right]}{\left(\mathrm{x}^{2}-\mathrm{r}^{2}\right)^{1 / 2}} \mathrm{D}(\mathrm{r}) \mathrm{rdr}
$$

which is the explicit formula, from which we can calculate $d(x)$, when $D(r)$ is a given piecewise continuously differentiable function. This result is for radially symmetric dose distributions in circular phantoms only.

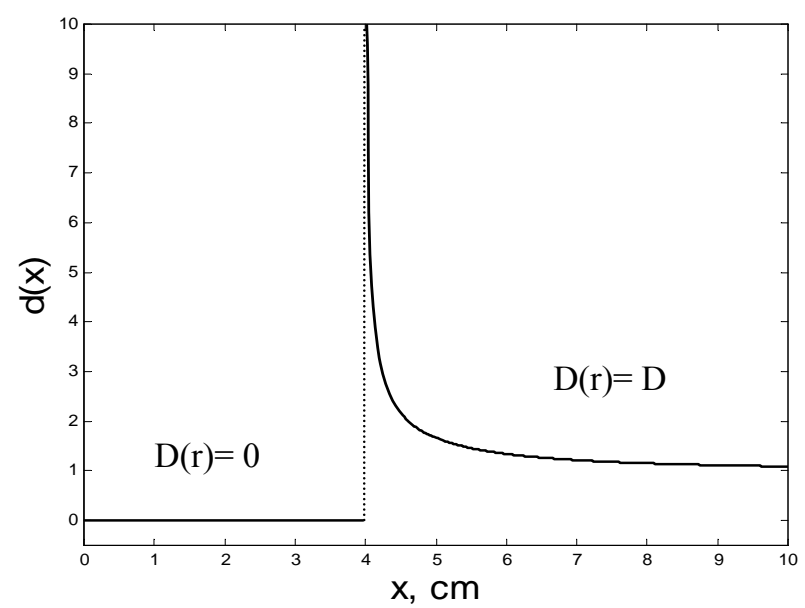

FIGURE 3-3. The lateral intensity profile across the radius of the cylindrical phantom received by explicit integral equation solution for $D(x)=D$ for $x \geq r_{0}$ and $D(x)=0$ for $x<r_{0}$. The values of $r_{0}$ and $R$ were $4 \mathrm{~cm}$ and $10 \mathrm{~cm}$, correspondingly, the linear attenuation coefficient $\mu=0.05 \mathrm{~cm}^{-1}$. The dose D was arbitrary set to 1 . 


\section{Implications in radiation therapy}

(1) 1st class of applications: central target volume with a uniform absorbed dose with smallest possible dose to surrounding healthy tissue, i.e.

$$
D(r)=\left\{\begin{array}{ccc}
D_{0} & \text { for } & r \leq r_{0} \\
0 & \text { for } & r>r_{0}
\end{array}\right.
$$

For such a central cylindrical target volume of radius r, using Eq. 3-8 the desired fluence variation becomes (Barth 1990, Brahme at al. 1982, Cormack 1987).

$$
d(x)=\left\{\begin{array}{cc}
D_{0} \cos (\mu|x|) & x \leq r_{0} \\
D_{0}\left[\cos (\mu x)-\frac{x \cos \left[\mu\left(x^{2}-r_{0}^{2}\right)\right]}{\left(x^{2}-r_{0}^{2}\right)^{1 / 2}}\right] & x>r_{0}
\end{array}\right.
$$

The fluence function for $x>r_{0}$ is not physical, the fluence is negative. Of course $d(x)$ should ideally be zero outside the interval and could be set to zero by adding a positive constant equal by modulus to the negative fluence. The lateral intensity variation for $\mathrm{x} \leq \mathrm{r}_{0}$ decreases slowly with the distance from the center, as is shown in Fig. 3-4.

The resultant dose distribution, which is different from the desired one (Eq. 3-9), in the cylinder outside $\mathrm{r}_{0}$ is obtained simply by Eq. 3-3:

$$
\mathrm{D}(\mathrm{r})=\left\{\begin{array}{cc}
\mathrm{D}_{0} & \mathrm{r} \leq \mathrm{r}_{0} \\
\frac{2 \mathrm{D}_{0}}{\pi} \arcsin \left(\frac{\mathrm{r}_{0}}{\mathrm{r}}\right) & \mathrm{r}>\mathrm{r}_{0}
\end{array}\right.
$$
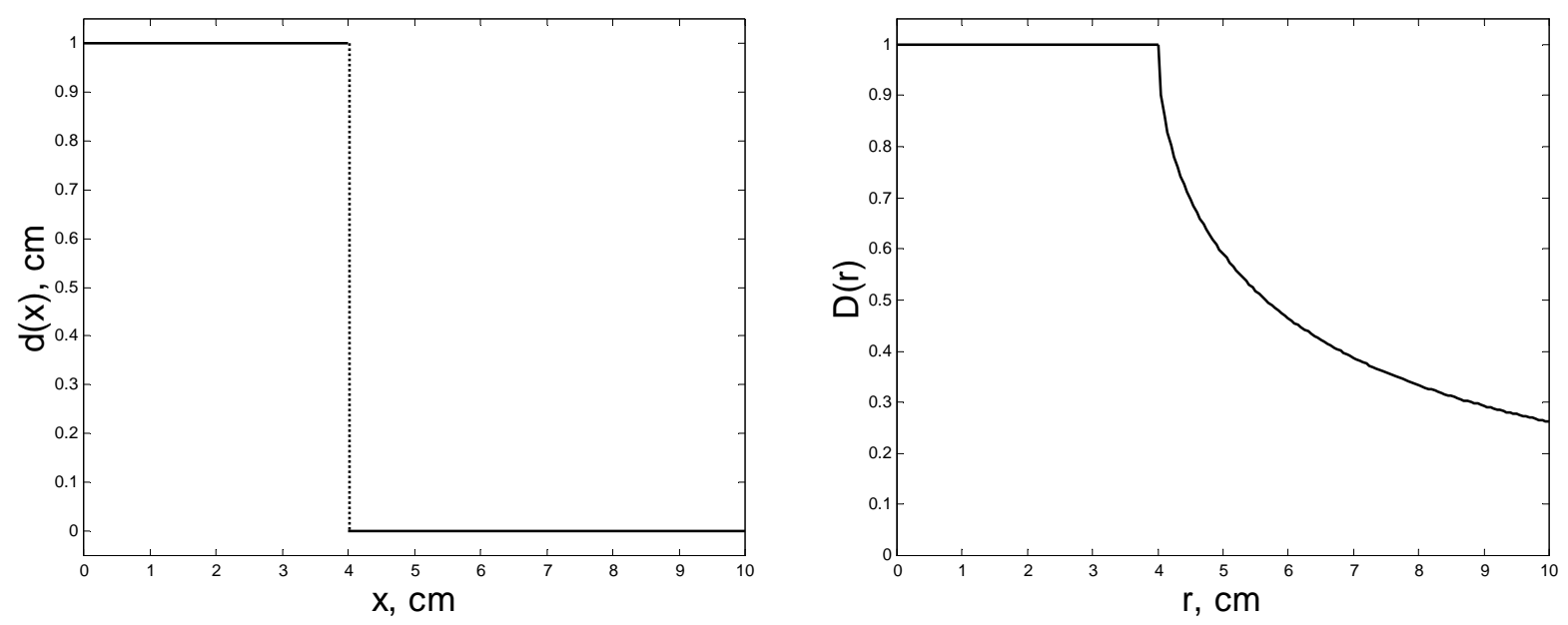

FIGURE 3-4. The deliverable lateral intensity profile (a) plotted according to Eq. 3-10 but with values of $\mathrm{d}(\mathrm{x})$ for $\mathrm{x}>\mathrm{r}_{0}$ set to zero and the resultant dose distribution (b) across the radius of the cylindrical phantom. The dose $D_{0}$ was arbitrary set to 1 . 
If the phantom absorption in the semicylindrical volume in front of the diameter is taken into account the dose profile in the incident photon beam, $d_{i n}(x)$, should be

$$
\mathrm{d}_{\mathrm{in}}=\mathrm{D} \cos \left(\mu_{\mathrm{p}}|\mathrm{x}|\right) \exp \mu\left[\left(\mathrm{R}^{2}-\mathrm{x}^{2}\right)^{1 / 2}-\mathrm{R}\right] .
$$

This correction can be included in the initial formulation of the problem as well. The intensity profile received by Eq. 3-11 is depicted in Fig. 3-5. As previously, it was assumed $r_{0}=4 \mathrm{~cm}$, while phantom radius $R=10 \mathrm{~cm}$. The dose value of $\mathrm{D}$ was arbitrary set to 1 and linear attenuation coefficient $\mu$ to $0.05 \mathrm{~cm}^{-1}$.

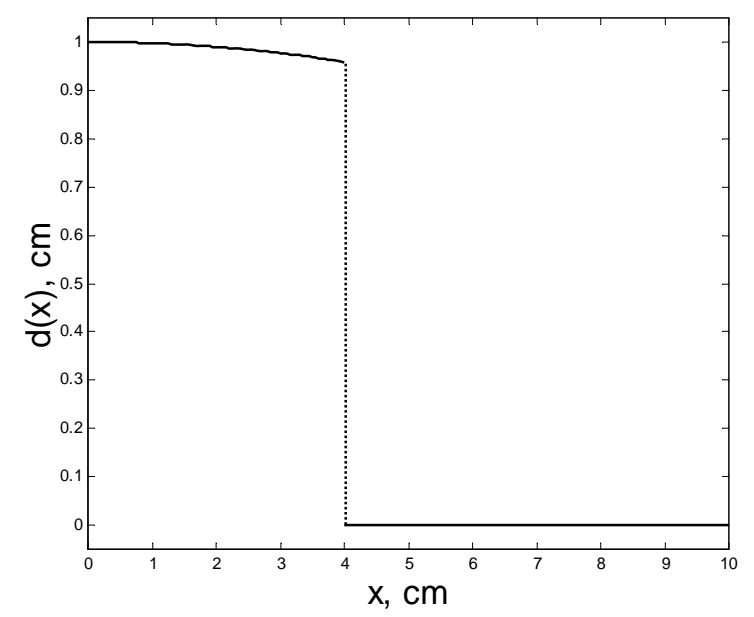

FIGURE 3-5. The lateral intensity profile across the radius of the cylindrical phantom received by taking into account the phantom absorption front of the diameter according to Eq. 3-11 for $\mathrm{D}(\mathrm{x})=\mathrm{D}$ for $\mathrm{x}<\mathrm{r}_{0}$ and $\mathrm{D}(\mathrm{x})=0$ for $\mathrm{x} \geq \mathrm{r}_{0}$. The dose $\mathrm{D}$ was arbitrary set to 1 .

(2) 2nd class of applications: central Organ at Risk (OAR) surrounded by the target volume with the desired dose $D(r)$ constant outside some radius $r_{0}$ and zero inside $r_{0}$

$$
D(r)=\left\{\begin{array}{lll}
0 & \text { for } & r \leq r_{0} \\
D & \text { for } & r>r_{0}
\end{array} .\right.
$$

The resultant dose distribution along the radius is given by Eq. 3-7 and depicted in Fig. 3-3. To get a uniform dose distribution outside $r_{0}$ the absorbed dose should theoretically increase to infinity as $r$ approaches $r_{0}$, as can be seen in Fig. 3-3. It should be noted, that the fluence for $\mathrm{x}<\mathrm{r}_{0}$ is non-physical. For real radiation delivery it should be set to zero, that will result in the dose behind the shield to be different from zero. Because of influence of scattered photons it is physically impossible to make $\mathrm{d}(\mathrm{x})=0$ inside $r_{0}$ especially as the dose level near $r_{0}$ is very high. Therefore, in practice, a compromise must be sought between the height of the peak dose value and the allowable scattered dose level inside $r_{0}$. In practice, as shown 
experimentally by Lax and Brahme (1982), a finite value at least a few times larger than the peripheral dose $d(R)$ at some large radius is sufficient. The use of the method in a clinical case with the high-gradient filters placed in such a way in order to produce the peripheral dose increase near the target volume outside the OAR was demonstrated by them.

\subsubsection{Dose distribution of reflection symmetry}

Cormack (1987) and Cormack and Cormack (1987) presented a generalization of the solution of the inverse problem to dose distributions with reflection symmetry, the axis of rotation being contained by the symmetry axis. In the cases of reflection symmetry, i.e., in which a dose distribution is symmetric about some axis (i.e. has reflection symmetry), the dose is proportional to the even part of the intensity expression $1 / 2 G(r, \theta) \exp \left\{-\mu\left(R^{2}-r^{2}\right)^{1 / 2}-\right.$ $p \sin (\theta-\varphi)\}$ (Barth 1990). Let us recall that the even part of some function is defined to be $1 / 2$ $\{\mathrm{f}(\mathrm{x})+\mathrm{f}(-\mathrm{x})\}$. The same result is achieved if we suppose parallel opposed beams incident on a target. Suppose a uniform circular phantom of radius R (shown in Fig. 3-6) is placed in parallel opposed beams of equal intensity monoenergetic $x$ rays. The origin is the center of rotation of two parallel opposed half-blocked beams. The line OS represents the half-beam block, so that the x-ray intensity is zero in the half-plane indicated by hash marks. In the unblocked region of the beam, intensity filters produce identical intensity profiles incident from both sides of the patient. The incident beams' intensities vary both with $r$, the distance to the line $\operatorname{OS}$ and $\theta$, the angle of the beam system. Each beam has the intensity $G(r, \theta) / 2$.

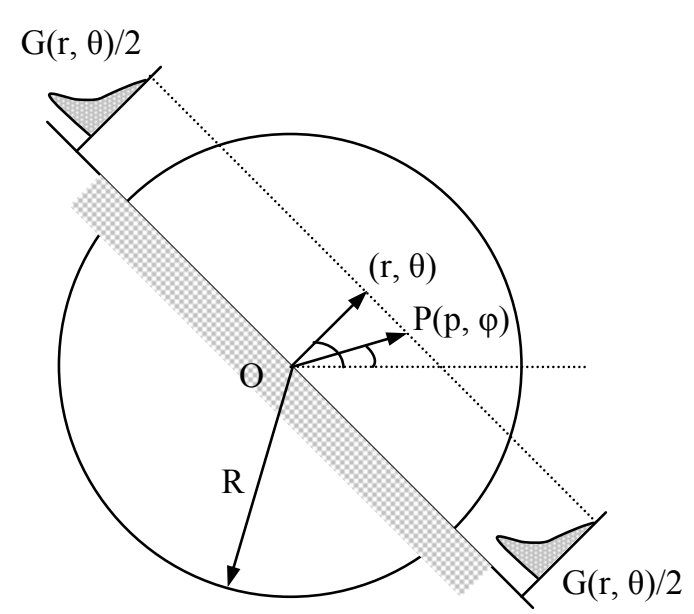

FIGURE 3-6. Parallel opposed beams of $\mathrm{x}$ rays are incident on a circular target of radius $R$ centered on the beams' axis of rotation. The beams are both halfblocked. In the unblocked region, the intensity of each beam has identical variation with $\mathrm{r}$ and $\theta$ given by $G(r, \theta) / 2$. A point within the patient is specified by polar coordinates $(p, \varphi)$. 
Then, the fluence of $\mathrm{x}$ rays in the material at point $\mathrm{P}$ will be given by

$$
\begin{aligned}
& \frac{1}{2} G(r, \theta)\left[\exp \left\{-\mu\left[\left(R^{2}-r^{2}\right)^{1 / 2}-p \sin (\theta-\varphi)\right]\right\}+\exp \left\{-\mu\left[\left(R^{2}-r^{2}\right)^{1 / 2}+p \sin (\theta-\varphi)\right]\right\}\right]= \\
& =G(r, \theta) \exp \left\{-\mu\left(R^{2}-r^{2}\right)^{1 / 2}\right\} \cosh \{\mu p \sin (\theta-\varphi)\}
\end{aligned}
$$

So,

$$
\mathrm{I}(\mathrm{x}, \mathrm{d}) \propto \exp \left(-\frac{\mu \mathrm{d}}{2}\right) \cosh (\mu \mathrm{x})
$$

where $\mu$ is the mass attenuation coefficient, $d$ is the material thickness, and $x$ is the distance from the center of the material. By ignoring scatter and the buildup region at the surface, the absorbed dose may be approximated to be proportional to the x-ray fluence.

Assuming the beam system completes a full rotation about point $O$, the integral intensity of the x-ray dose at a point $\mathrm{P}$ within the patient, $D(p, \varphi)$, is given by (Cormack 1987)

$$
\mathrm{D}(\mathrm{p}, \varphi)=\frac{1}{\pi} \int_{\varphi-(\pi / 2)}^{\varphi+(\pi / 2)} \mathrm{G}(\mathrm{r}, \theta) \exp \left[-\mu\left(\mathrm{R}^{2}-\mathrm{r}^{2}\right)^{1 / 2}\right] \cosh \{\mu \mathrm{p}[\sin (\theta-\varphi)]\} \mathrm{d} \theta
$$

where $r=p \cos (\theta-\varphi)$ and the factor $\exp \left[-\mu\left(R^{2}-r^{2}\right)^{1 / 2}\right] \cosh \{\mu p[\sin (\theta-\varphi)]\}$ is the result of beam attenuation within the patient. The problem is to find $G(r, \theta)$ from a specified $D(p, \varphi)$.

The problem, Eq. (3-16), is simplified by defining a new quantity $F(r, \theta)=G(r, \theta) \exp [-\mu$ $\left.\left(R^{2}-r^{2}\right)^{1 / 2}\right]$ and changing the variable of integration to yield $\mathrm{D}(p, \varphi)$

$$
\mathrm{D}(\mathrm{p}, \varphi)=\frac{2}{\pi} \int_{0}^{\mathrm{p}} \mathrm{F}(\mathrm{r}, \theta) \frac{\cosh \left[\mu\left(\mathrm{p}^{2}-\mathrm{r}^{2}\right)^{1 / 2}\right]}{\left(\mathrm{p}^{2}-\mathrm{r}^{2}\right)^{1 / 2}} \mathrm{dr}
$$

which coincides with the definition of the problem Eq. 3-3 derived by Brahme (1982) and Barth (1990).

The solution was derived using an expansion of the dose and intensity distributions into circular harmonics, which leads to the use of Chebyshev polynomials (Cormack 1987). It has also been shown that this approximation has a well-established mathematical theory. Each side of Eq. (3-17) is expanded in terms of circular harmonics,

$$
\mathrm{F}(\mathrm{r}, \theta)=\sum_{-\infty}^{\infty} \mathrm{F}_{1}(\mathrm{r}) \mathrm{e}^{\mathrm{i} l \theta} \quad \text { and } \quad \mathrm{D}(\mathrm{p}, \varphi)=\sum_{-\infty}^{\infty} \mathrm{D}_{1}(\mathrm{r}) \mathrm{e}^{\mathrm{i} l \varphi}
$$


followed by variable transformations

$$
\mu \mathrm{r}=\mathrm{s}, \quad \mu \mathrm{p}=\mathrm{q}, \quad \mathrm{F}_{1}(\mathrm{~s} / \mu)=\mathrm{f}_{1}(\mathrm{~s}), \quad \mathrm{D}_{1}(\mathrm{q} / \mu)=\mathrm{d}_{1}(\mathrm{q}),
$$

to yield a term by term relation:

$$
d_{1}(q)=\frac{2}{\pi} \int_{0}^{q} f_{1}(s) T_{1}\left(\frac{s}{q}\right) \frac{\cosh \left[\left(q^{2}-s^{2}\right)^{1 / 2}\right]}{\left(q^{2}-s^{2}\right)^{1 / 2}} d s
$$

where $T_{l}(x)$ is the Tschebycheff polynomial of the first kind of order $l$. When the transformations involved in Eq. 3-19 are carried out for $\mu$ typical of treatment energies, coordinates within the patient will correspond to $|p|<1$ and $|s|<1$.

The resulting equation must be satisfied term by term. Each term represents a single beam profile that is used for the entire treatment arc. The lowest-order term in the expansion $(l=0)$ would be the only nonzero term in the case of circular symmetry. A fortuitous feature of the equation allows the solution for $l=0$ to yield solutions for distributions with axial symmetry $(l=1)$, and beam profiles have been determined for a small number of such dose distributions Cormack and Cormack (1987). Unfortunately, analytical solutions to Eq. 3-20 for higher-order terms $(l=1)$, or general radial variation, have not been found.

\subsubsection{Arbitrary dose distribution}

Barth (1990) extended the formalism to convex phantoms of arbitrary cross section. He showed that the fluence (intensity) $I_{0}(x, \alpha)$ necessary to produce some dose distribution $D(r)$ in an arbitrary convex region, characterized by the function $R(x, \alpha)$, can be obtained by multiplication of the fluence for circular phantom of radius $k$ (by which the given region is fictitiously considered to be enclosed) by the factor $\exp \left[\mu\left(R^{2}(x, \alpha)-x^{2}\right)^{1 / 2}\right]$, where $R(x, \alpha)$ is as shown in the Fig. 3-7.

Additionally, relations necessary to produce circularly symmetric dose distributions about any point without the phantom were obtained. Thus non-radially symmetric dose distribution can be obtained by decomposing it into $\mathrm{N}$ non intersecting dose distributions of different radii $r_{i}$ each centered about a point $\left(\rho_{i}, \gamma_{i}\right)$, as shown in Fig. 3-8. Thus the fluence function necessary to create the dose distribution after one rotation of the treatment beam about the origin becomes: 


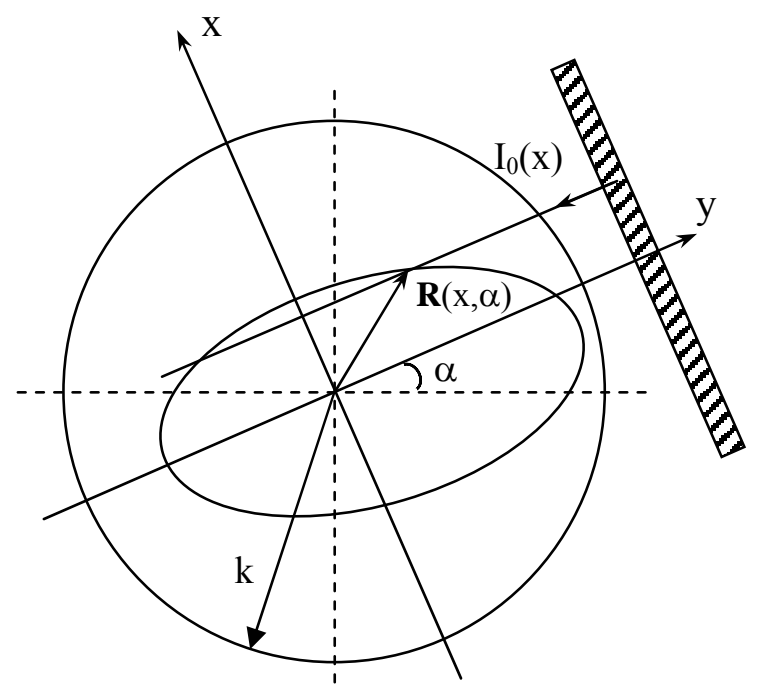

FIGURE 3-7. A convex phantom of arbitrary cross-section, characterized by function $\mathrm{R}(\mathrm{x}, \alpha)$ enclosed within a circular region of radius $\mathrm{k}$.

$$
\mathrm{I}_{\text {Total }}(\mathrm{y}, \alpha)=\sum_{\mathrm{i} \in \Gamma} \mathrm{I}\left(\mathrm{x}, \rho_{\mathrm{i}}, \gamma_{\mathrm{i}}, \alpha\right)
$$

Kooy and Barth (1990) evaluated dose distributions obtained with the inverse method by use of Monte Carlo methods and numerical integration. The results show correctness of the formalism for a dose specification to a single circular region at an arbitrary position in a 2dimenssional phantom. However, the approach of Barth (1990) to more complicated noncircular regions produces artifacts in the physical dose distribution, but they can be minimized, using the method presented by Kooy and Barth (1990), and the formalism can still yield practical results.

Since target volume is embedded in the phantom, photons need to traverse a non-target region to reach the target region, and hence the dose is deposited outside the target region. The beam fluence function includes positive and negative fluencies. The negative fluence

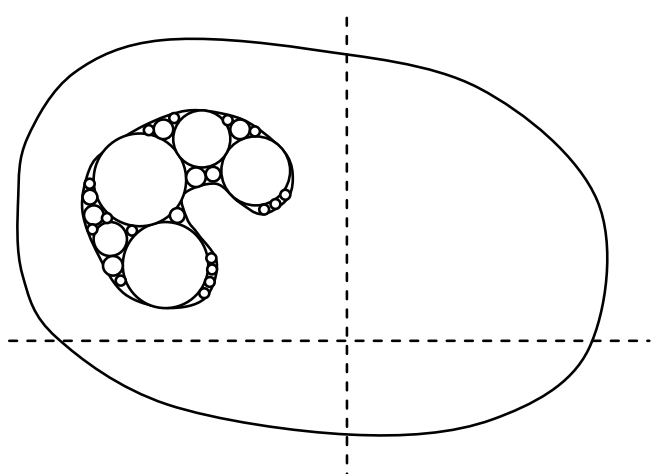

FIGURE 3-8. A convex phantom of arbitrary cross-section with an orbitrary concave dose distribution. The arbitrary concave dose distribution is buildt up out of $\mathrm{N}$ small radially symmetric non-intersecting dose distributions of different radii $r_{0 i}$ each centered about a point $\left(\rho_{i}, \gamma_{i}\right)$, where $i=1, \ldots, N$. 
exactly compensates for this dose, resulting in a net zero dose in the region outside the target region (Kooy and Barth 1990). However, physically deliverable fluences are positive. Such fluences for several target volumes are obtained after the final summation of Eq. 3-21 by setting the remaining negative fluences to zero. Unfortunately, when this is done there is a price to be paid: the new physical fluence function no longer produces the ideal dose distribution.

The problem of non-negativity, which is of great interest, was mentioned by all authors dealing with inverse treatment planning. The beam fluence function includes positive and negative fluencies. The negative fluence exactly compensates for this dose, resulting in a net zero dose in the region outside the target region (Kooy and Barth 1990). Thus, some distribution the radiation fields cannot be delivered by any real radiation fields, calculated directly from a desired dose.

The methods to eliminate negative values of the beam profile proposed by Bortfeld and Boyer (1995) are:

- First, simply to reset to zero all points of the profile at which negative values were computed. (does not cause overdosage, may result in intolerable dose inhomogeneities).

- Second, a positive constant can be added to each profile sufficient to maintain nonnegative values all along profile. (dose homogeneity is preserved, high load on normal tissues).

It may be favorable to use a combination of both of these methods.

Analytic solutions to Eq. (3-17) exist for a few dose distributions, each with a single nonzero term with $|l| \leq 1$, but do not exist for general dose distributions having either a general radial dependence or terms in the expansion with $|l| \geq 2$ (Cormack 1987, Cormack and Cormack 1987). By moving from a purely analytic approach to a numerical solution of the analytic problem, the problem may be solved for arbitrary order circular harmonics and for arbitrary radial variation having a given angular variation, thus greatly expanding the solution set of earlier works (Cormack 1998).

The numerical solution is constrained to remain non-negative. If a prescribed dose distribution is physically unattainable, the technique can produce a physically achievable beam profile that will approximate a prescribed one. The analytic solution is simply cropped to zero at all points where the solution is negative. 
A very interesting example of the numerical solution of the problem, included in the work of Cormack (1998), was an unachievable distribution of two concentric annular high dose regions. The desired dose was specified to be

$$
\begin{aligned}
\mathrm{D}(\mathrm{p}, \varphi) & =0, & & 0<\mathrm{p}<0.2 \\
& =1, & & 0.2<\mathrm{p}<0.4 \\
& =0, & & 0.4<\mathrm{p}<0.6 \\
& =1, & & 0.6<\mathrm{p}<0.8 \\
& =0, & & 0.8<\mathrm{p} .
\end{aligned}
$$

This distribution is clearly unphysical. While the beams may be chosen to deliver no dose to the region $p<0.2$, to deliver the dose to the region $0.2<p<0.4$ requires the entrance and exit dose to pass through all radii greater than 0.4 . The analytic solution and numerical solutions are both shown in Fig. 3-9(a). The discrepancy between the two becomes apparent for radii greater than $p>0.4$. Because the analytic solution incorporates negative fluence, the solution yields a greater fluence for the outer high dose region. The dose profiles that would result from trying to use these profiles are shown in Fig. 3-9(b). The analytic solution is simply cropped to zero at all points where the solution is negative. It either case, the sharp dose falloff in the intermediate region is unattainable, and the geometric falloff is observed. However, unlike the analytic solution, the numerical solution recovers once the dose distribution is physically achievable. A simple cropping of the analytic solution would overdose the outer region, while the numerical solution recovers to produce the desired dose.
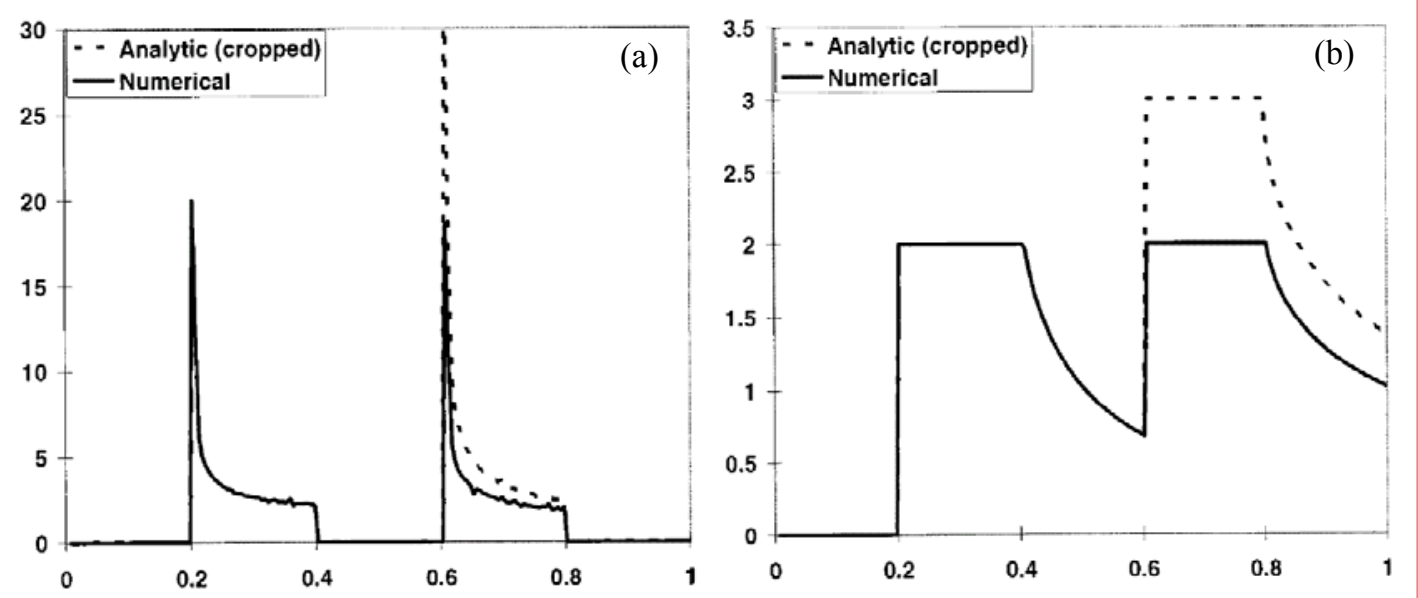

FIGURE 3-9. (a) Beam profiles calculated for the physically unachievable double ring geometry of Eq. 22. The analytical solution demands negative beam intensities. Once negative fluence is set to zero, the remaining solution includes an excess fluence at larger radii. However, the numerical solution incorporates the non-negativity constraint. (b) Te dose profile that would arise from using the physically realizable form the solution above. The falloff in the region $0.4<p<0.6$ is avoidable due to the entrance dose to the inner region. The numerical solution recovers to yield the desired solution once it is again physically achievable, while simply cropping the analytic solution will not yield the desired distribution (Cormack 1998). 
Using a numerical approach allows the incorporation of physical constraints that previously were not able to be considered. The beam profiles calculated by the numerical method agree well with analytic solutions where available, and thy produce the desired distributions for higher-order terms, where analytic solutions are not available. This technique also offers a straightforward way in which to model additional beam characteristic, such as scatter effect. A slight modification of the numerical evaluation would be required to compensate for the additional dose delivered to smaller radii from the scatter term, but given that the primary is the dominant contribution to the dose, this should not lead to convergence problems.

The first complete solution for the inverse problem of x-ray rotation therapy in analytical form was presented by Oelfke and Bortfeld (1999). The applied strategy for the solution of the inverse problem was based on two steps. First, a polynomial expansion of dose and fluence profiles is used to obtain a general solution of the inverse problem. Then, the derived results are employed to obtain fast and more practically applicable solution in terms of Hankel and Fourier transforms.

So far it has been assumed throughout the literature (Cormack 1987, Cormack and Cormack 1987, Barth 1990 and Bortfeld and Boyer 1995) that only fluence profiles that are identical for opposite directions of beam incidence constitute a valid solution of inverse problem. This assumption is naturally inherent for approaches originating from the problems of CT image reconstruction, where absorption profiles measured at opposite CT angles always coincide. Oelfke and Bortfeld (1999) disposed of this constraint to fully exploit the degrees of freedom for intensity modulation in rotation therapy.

The next milestone in the evolution of inverse planning in 1988 was also by Brahme (1988), in which was explained the notion of creating fluence profiles from dose distributions by first inspecting the dose distribution in dosespace, then deconvolving the point-spread dose kernel from this to create the density of fluences required. The next step was to backproject the density of fluence back into fluence space to create the fluence profiles. Then, from the profiles so generated, the deliverable dose distributions could be formed by a process of projecting the fluence back into dosespace. The procedure is schematically illustrated in Fig. $3-10$.

The projection and backprojection processes both involved exponential depth operations. However, projection and backprojection are not inverse processes and so the technique was only approximate. Also, the process could generate negative fluences and so required some form of truncation to zero or the addition of constant terms in fluence space. 


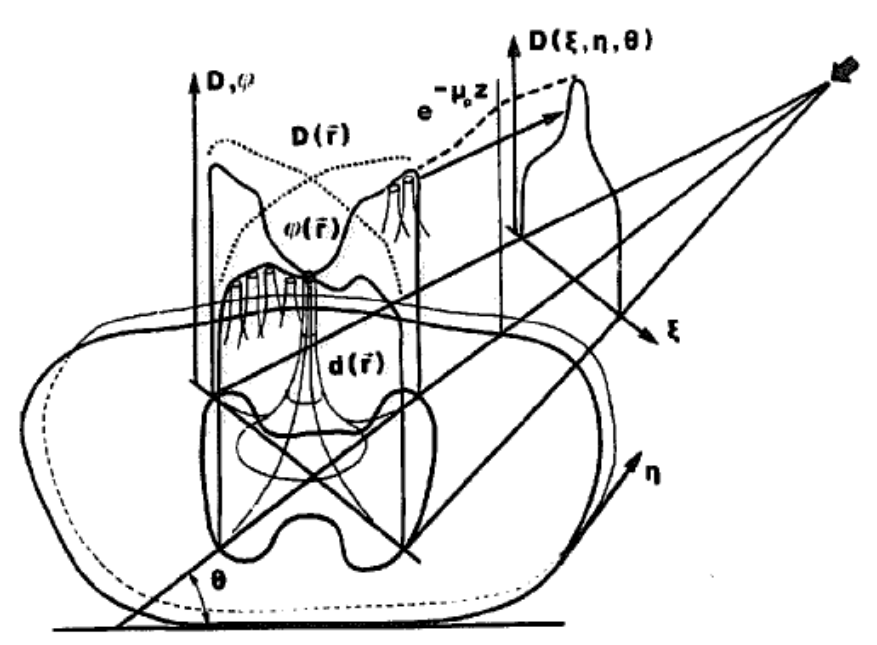

FIGURE 3-10. Schematic illustration of the decomposition of a desired dose distribution $\mathrm{D}(\mathbf{r})$ in a density distribution $\varphi(\mathbf{r})$ of convergent point irradiation distributions $\mathrm{d}(\mathbf{r})$. The optimal incident beam during moving beam therapy $\mathrm{D}(\xi, \eta, \theta)$ is obtained by inverse back-projection of the point irradiation density $\mathrm{d}\left(\mathbf{r}_{\mathbf{s}}-\mathbf{r}\right) \varphi(\mathbf{r})$ into the effective radiation source (Brahme 1988).

The work was a landmark because it established the idea of this kind of planning viewed as a form of inverse computed tomography.

\subsection{SIMILARITY OF INVERSE PROBLEM IN RADIATION THERAPY WITH IMAGE RECONSTRUCTION IN CT}

The optimal irradiation techniques have many similarities with the back-projections used in computed tomography (CT), as seen from Fig. 3-11. The similarity between CT and conformation therapy has been noted in many published papers and notes (Brahme et al. 1982, Brahme 1988, Webb 1989). However, the methods for the calculation of the Intensity Modulated Fields (IMF) used by these authors are different from the methods which are usually used in CT. Brahme (1988) uses a 2D convolution technique and Webb (1989) uses

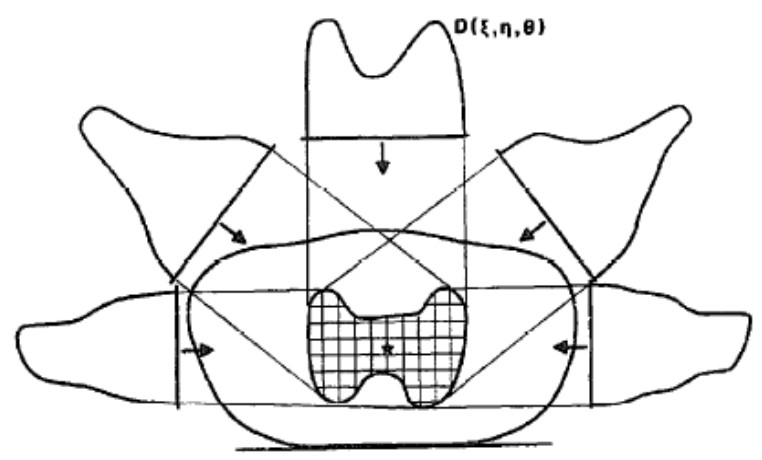

FIGURE 3-11. Schematic illustration of the type of dose delivery that will give the desired dose distribution in the target volume (shaded) and at the same time minimal dose to surrounding normal tissues (Brahme 1988). 
the method of simulated annealing. Bortfeld et al. (1990) presented methods for the calculation of the IMF based on two algorithms which are well established in CT, and which allow a very fast calculation and optimization of the IMF. He compares the new method of inverse planning of radiation treatment to the method of reconstructing a $3 \mathrm{D}$ image (rather, a density distribution of the tissue) from its projections, which is known from computer tomography (CT). Both of these methods are closely related: the projections in CT correspond to the IMF in conformation therapy (Fig. 3-12) and the density distribution corresponds to the dose distribution in the patient. The problem in conformal therapy is the following: given the prescribed dose distribution in the patient, calculate the IMF so that the resulting dose distribution comes closest to the prescribed distribution. This is indeed the inverse problem to CT: given the measured projections, calculate the tissue density distribution so that its projections come closest to the given projections. The main processes of an image reconstruction and of conformal radiotherapy are compared in Fig. 3-13.

Computed tomography

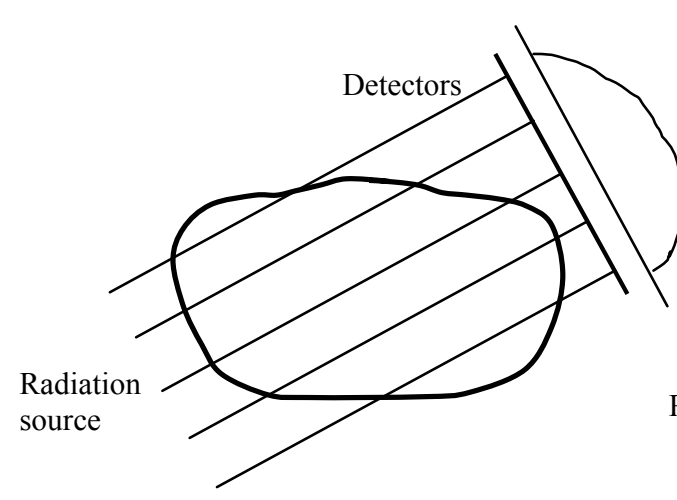

Conformal radiotherapy

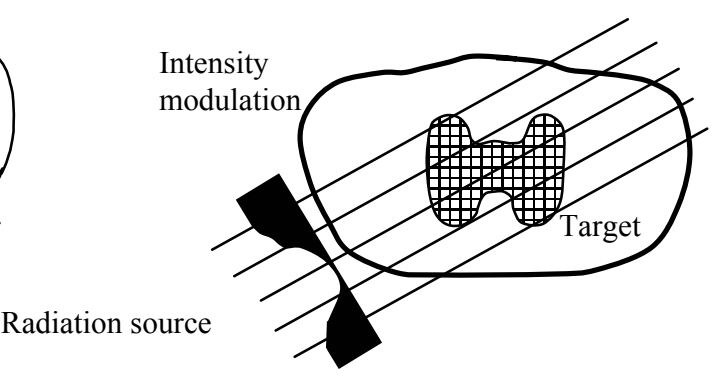

FIGURE 3-12. Schematic sketch of comparing of (a) computed tomography and (b) conformal radiotherapy. The beam geometry is assumed to be parallel.

The main difference is that the negative valued filters used in CT reconstruction cannot be used in radiation therapy, as the incident dose distributions by necessity are non-negative. The practical consequence of this fact is that arbitrary dose distributions cannot be delivered whereas arbitrary attenuation patterns can be determined using CT (Brahme 1988). For example, it is not possible to give a high dose to a volume in the patient and zero dose everywhere else.

Later on, Bortfeld and Boyer (1995) have presented a more detailed study of the similarity of the inverse planning in conformal radiotherapy and CT image reconstruction and applied Radon transform to the field of radiotherapy. This resulted in a projection-filtering algorithm for the calculations of optimal beam profiles. 


\section{Methods of image reconstruction}

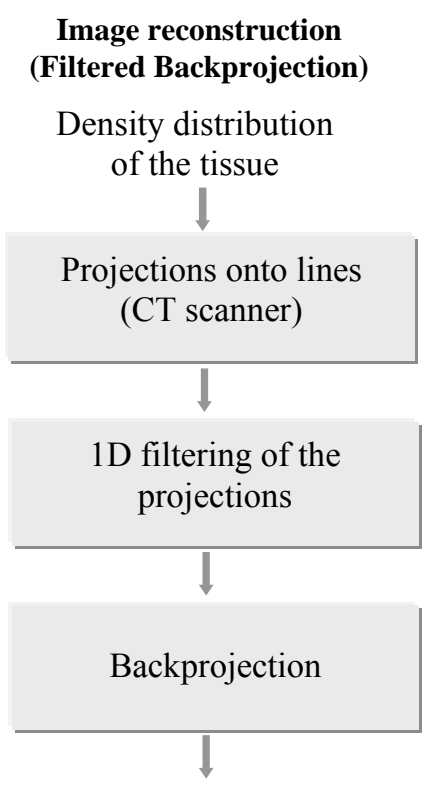

Set of 2D slice images

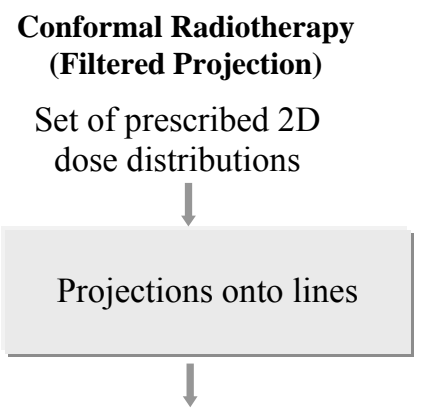

1D filtering of the projections

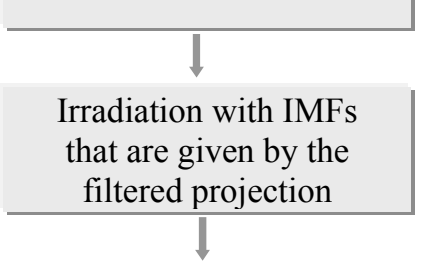

Dose distribution in the tissue

FIGURE 3-13. Comparison of the steps of image reconstruction and conformal radiotherapy (Bortfeld 1990).

Also Cormack (1987) had pointed out than the problem definition in radiotherapy in form of

$$
\mathrm{D}(\mathrm{p}, \varphi)=\frac{1}{2 \pi} \int_{0}^{2 \pi} \mathrm{G}(\mathrm{p} \cos (\theta-\varphi), \theta) \mathrm{d} \theta
$$

represent $D$ as the integrals of $G$ over circles through the origin and it is thus a problem of Radon's type that is the problem of recovering a function from its integrals over curves in the plane. The Radon problem of integrals over circles through the origin has a history over decades of years. The first treatment of the problem has been given by George Birkhoff in 1940 (Birkhoff 1940).

Using BRL approximation (refer to sect. 3.1) and that the attenuation of the beam is purely exponential, the dose distribution of a single beam is

$$
\mathrm{D}_{\phi}\left(\mathbf{r}^{\prime}\right)=\mathrm{G}\left(\mathrm{x}^{\prime}, \phi\right) \mathrm{e}^{-\mu \mathrm{z}^{\prime}}
$$

where $G\left(x^{\prime}, \varphi\right)$ is the beam profile, as shown in Fig. 3-14. In this representation the single beam dose distribution can be considered the back-projection of the beam profile with the exponential attenuation. 


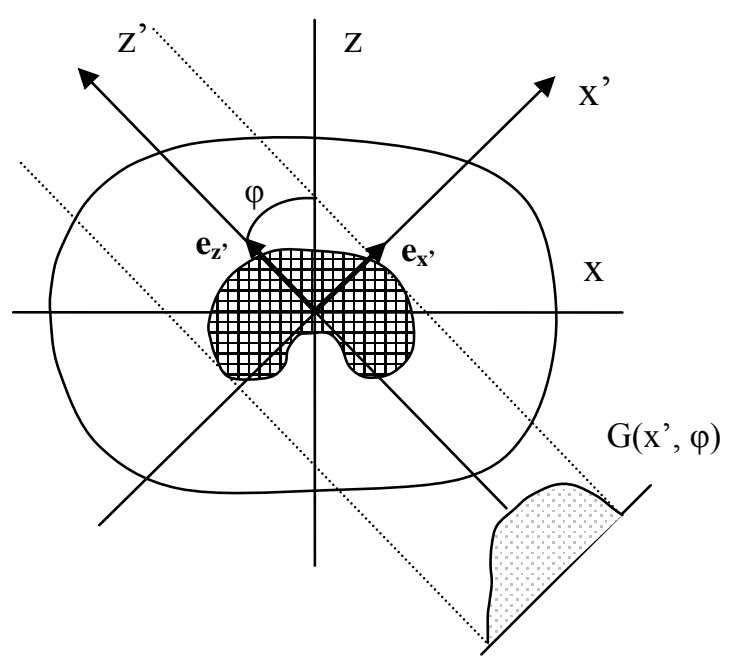

FIGURE 3-14. Schematic representation of the parameters used. Each beam is directing along a $z^{\prime}$ axis that has been rotated with the (x', z') system about the origin through an angle $\varphi$.

The total dose distribution after a complete rotation of the beam by $2 \pi$ is given by the integral

$$
\mathrm{D}(\mathrm{r})=\int_{0}^{2 \pi} \mathrm{D}_{\phi}\left(\mathbf{r}^{\prime}\right) \mathrm{d} \phi
$$

The relationship between the total dose distribution and the beam profiles can formally be expressed though a functional operator $\mathcal{B}_{\mu}$, which is defined according to the above equation:

$$
\mathrm{D}(\mathrm{r})=\mathscr{B}_{\mu}\left\{\mathrm{G}\left(\mathrm{x}^{\prime}, \varphi\right)\right\}
$$

If the inverse operator $\mathcal{B}_{\mu}{ }^{-1}$ were known, the required beam profiles could be calculated for arbitrary prescribed dose distribution:

$$
\mathrm{G}\left(\mathrm{x}^{\prime}, \varphi\right)=\mathcal{B}_{\mu}^{-1}\{\mathrm{D}(\mathrm{r})\}
$$

There is a close relationship between $\mathscr{B}_{\mu}$ and the attenuated of exponential Radon transform $R$. The operator $R$ can be considered a projection operator with exponential weighting. It is defined by its application to an arbitrary function $D(r)$ as follows:

$$
\mathbb{R}_{\mu}\{\mathrm{D}(\mathrm{r})\}=\int_{-\infty}^{\infty} \mathrm{D}\left(\mathrm{x}^{\prime} \mathbf{e}_{\mathbf{x}^{\prime}}+\mathrm{z}^{\prime} \mathbf{e}_{\mathbf{z}^{\prime}}\right) \mathrm{e}^{\mu \mathrm{z}^{\prime}} \mathrm{dz}
$$

Using a relationship between $\mathscr{B}_{\mu}$ and $\mathbb{R}_{\mu}$ and some successive transformations (Bortfeld and Boyer 1995), it makes possible to calculate beam profiles for arbitrary prescribed dose distribution:

$$
\mathrm{G}(\mathrm{x}, \varphi)=F_{1}^{-1}\left\{\mathcal{K}_{\mu} F_{1}\left\{R_{\mu}\{\mathrm{D}(\mathrm{r})\}\right\}\right\} .
$$


Reading this operator equation from right to left, one obtains the following "recipe" for solution of the inverse problem (Bortfeld and Boyer 1995):

1. Exponential Radon transformation (projection) of the desired dose distribution yields projection profiles

$$
\mathrm{G}^{*}\left(\mathrm{x}^{\prime}, \varphi\right)=\left\{\mathbb{R}_{\mu}\{\mathrm{D}(\mathrm{r})\}\right.
$$

2. One-dimensional Fourier transformation of the projection profiles yields frequency domain profiles

$$
\mathrm{G}^{*}(\xi, \varphi)=F_{1}\left\{\mathrm{G}^{*}\left(\mathrm{x}^{\prime}, \varphi\right)\right\}
$$

3. Filtration in the frequency domain by multiplication of $\mathrm{P}^{*}(\xi, \varphi)$ with the filter function $\mathrm{K} \mu\left(\xi^{\prime}\right)$ yields

$$
\mathrm{G}\left(\xi^{\prime}, \varphi\right)=\mathcal{K}_{\mu}\left(\xi^{\prime}\right) \cdot \mathrm{G}^{*}\left(\xi^{\prime}, \varphi\right)
$$

4. Inverse Fourier transformation (one-dimensional) finally results in the beam profiles

$$
\mathrm{G}\left(\mathrm{x}^{\prime}, \varphi\right)=F_{1}^{-1}\{\mathrm{G}(\xi, \varphi)\} .
$$

It was shown by Bortfeld and Boyer (1995) that Radon transform theory and particularly the method of projection filtering can be successfully applied to radiotherapy planning. The strategy to ensure non-negativity of the beam profiles generally provided satisfactory approximations of the desired ideal dose distribution. The applicability of this approach to the treatment panning for some clinical cases was studied and discussed by Mohan at al. (1994). Braunstein and Levine (1999) extended the theory of tomographic dose reconstruction. One of the main issues considered was the number of beams required to reconstruct a given prescribed dose, the existence of preferred beam directions which may be used to reduce the number of beams sufficient to achieve a certain level of conforming dose.

However, it is generally difficult to consider certain optimization criteria in the calculation criteria. Because these optimization criteria can hardly be considered by direct (non-iterative) calculation methods such as the projection filtering described, iterative optimization techniques are more often employed for the practical solutions of the inverse problem of the radiotherapy planning. Nevertheless, projection filtering can provide an excellent initial guess for the iteration process, hence reducing the number of iteration steps (Bortfeld et al. 1990, Bortfeld and Boyer 1995).

Optimization criteria can be as following: 
(i) The dose applied to the target should be very close to the prescribed dose.

(ii) The dose should be homogeneously distributed across the target.

(iii) The dose to particular organs at risk which are sensitive to radiation should be less than a tolerable maximum value.

(iv) In the tissue surrounding the target, the dose should be low.

Today these criteria are frequently more quantitatively defined using 'dose volume histograms'. In order to obtain a mathematical formulation of the problem we have to define an 'objective function' which is minimized during the optimization process.

\subsection{GOING BEYOND THE DIRECT INVERSE PROBLEM}

Formally, a 3D dose distribution $D$ is related to a set of beamweights $w$ via a matrix operator $A$ through:

$$
\mathrm{D}=\mathrm{A} \cdot w
$$

where $w$ is the vector of individual beamweights and $A$ is the matrix linking each dose-space element to each beam-space element. The elemental entries into $A$ depend on the physics of photon- tissue interaction and can be pre-calculated using either simple equations, or convolution and superposition algorithms or at best full Monte Carlo calculations. The "inverse problem" is that we can specify the required dose prescription $D$, we know $A$ and we require to calculate $w$. At first sight it might seem logical to perform a simple inversion:

$$
\mathrm{w}=\mathrm{A}^{-1} \cdot \mathrm{D}
$$

However, this is generally not done nowadays in inverse treatment planning because: (i) it would generate some negative beamweights; (ii) it would require the construction, storage and inversion of a too large matrix A; (iii) it would take too long and; (iv) it would provide no method to control the behavior of the beamweight vector $w$. The matrix $A$ is "ill-conditioned". This means that there are a very large number of different vectors $w$ that when operated on by matrix $A$ give the $3 \mathrm{D}$ dose distribution $D$ (Webb 2003).

The direct inverse approach suffers from three basic limitations leading to the optimization strategy of inverse planning (Oelfke and Bortfeld 2001):

- Physical limit

It results if the specified dose distribution physically cannot be realized for the radiation modality considered. As an example, let us consider the dose gradient between the boost volume and the 
remaining target. For a steeper dose gradient, the central fluence related to the boost volume might even cause an excess dose within the remaining target. The formal, mathematical solution of the inverse problem would suggest to subtract the excess dose by a negative fluence amplitude, which naturally cannot be done.

- Practical limit

To achieve a steep dose gradient at the edge of the central OAR, one has to deliver an extremely large fluence to the target area adjacent to the OAR, while the residual target receives significantly lower photon exposure. This enormous dynamic range of the photon fluence, however, cannot be delivered with most dose delivery systems.

- Clinical limit

Simple relationships such as the one between irradiated volumes, dose level, and a probable radiationinduced complication are very hard to establish for many organs at risk.

For certain clinical situations it is not sufficient to specify the objectives of optimization purely in terms of the desired pattern of dose (Mohan et al 1994). The problem of specifying the optimal dose distribution for a patient combined with the difficulty of estimating practically and physically achievable dose patterns leads to the optimization approach of inverse planning. The objectives have also to include volume-dose effects and biological indices. The extension of direct inverse planning to an optimization strategy has leaded to the currently employed concept for clinical IMRT planning.

Task, to be solved even before the inverse problem can be tackled, consists of finding a mathematical description of optimal dose distributions such that different treatment plans can be ranked according to their merits (Oelfke and Bortfeld 2001). This creation of dose-specific ranking criteria leads to the term of the objective function (OF) for the optimization of treatment plans. The objective function employs criteria-gathered clinical experience to specify the expectations for a treatment plan. Its definition is one of the central research topics for the clinical application and evaluation of IMRT.

The clinical merit of the dose distributed in targets and organs at risk is expressed by a single scalar function $O F\left(D(\mathbf{r})\left\{w_{i}\right\}\right)=O F\left(\left\{w_{i}\right\}\right)$. This objective function measures the violation of desired clinical objectives for a specific treatment plan. The value of $O F(\{w i\})$ therefore indicates the quality of a given treatment plan; an optimal set of treatment parameters would correspond to a minimum of the objective function. Physical objective functions $O F\left(\left\{w_{i}\right\}\right)$ aim to describe the clinical objectives of a radiation treatment in terms of physical parameters such as dose levels and irradiated volumes per organ. The advantage of this approach is that these physical quantities can be experimentally verified with high reliability through refined dosimetric procedures. 
The design of a physical objective function consists of three steps (Oelfke and Bortfeld 2001). First, a set of dose volume histogram DVH constraints is defined for any organ or tissue included in the optimization process. The clinical parameters for these conditions consist of tissue-specific dose levels and irradiated volumes, which are derived from clinical experience. Second, a mathematical measure $O F_{k}\left(\left\{w_{i}\right\}\right)$ for the violation of each individual constraint $k$ is defined. Third, for each constraint, a weighting factor $v_{k}$, the so-called penalty factor, is introduced to adjust the relative importance of the constraints according to the preference of the radiation oncologist; the total objective function of the desired treatment takes the form:

$$
\mathrm{OF}\left(\left\{\mathrm{w}_{\mathrm{i}}\right\}\right)=\sum_{\mathrm{k}_{1}}^{\mathrm{N}_{0}} \mathrm{v}_{\mathrm{k}} \mathrm{OF}_{\mathrm{k}}\left(\left\{\mathrm{w}_{\mathrm{i}}\right\}\right)
$$

with $N_{0}$ as the total number of defined dose-volume constraints.

The optimum plan is the best that could ever be obtained for treating a particular patient with a particular external shape, location of disease and arrangement of internal organs. Actually, this optimum plan is unachievable. To arrive at the optimum plan one would have to investigate the use of: (i) all types of irradiation (protons, carbon ions, photons...); (ii) of all energies (continuous not just those we have available); (iii) all possible numbers of beams from 1 to infinity; (iv) all possible ranges of fluence levels; (v) all possible beam geometry shapes; (vi) all possible fractionation schemes ... and so on. It is totally apparent that as "optimizers" we cannot and do not do this. We are constrained by: (i) the beams available on our machines; (ii) the need to keep the number of beams deliverable within some specified delivery timeslot; (iii) the delivery mechanics available to us (which links in to the fluence level issue); (iv) the collimation available from the machine; (v) the need to treat in the daytime not at night; (vi) the time available for planning... and so on. In practice the planner and doctor have already considerably reduced the optimization search space by making a priori choices, e.g. the choice to work with photons at $6 \mathrm{MV}$, with say 5 beams from certain specified directions, with a preset number of fluence levels, with the MLC of a particular manufacturer, with a specified fractionation scheme. Then all that is left to optimize is the beam profile subject to the constraints set. When we write of "optimization" we mean most certainly "constrained optimization" (Webb 2003). Most studies of "optimization" have concentrated on finding the best beam directions and best modulation.

Commonly used techniques for inverse treatment planning include computer simulated annealing (Webb 1989), iterative approaches (Bortfeld et al 1990, Holmes and Mackie 1994), filtered back-projection and direct Fourier transformation (Bortfeld et al 1990, Brahme 1988). The review of the inverse planning algorithms for external beam radiation therapy was done by Chui and Spirou (2001). Simulated annealing is a powerful approach for inverse treatment planning, but a drawback to its clinical implementation is the excessive computation time. Inverse treatment planning based on filtered back-projection and direct Fourier transformation has difficulty in handling the negative 
fluence problem, and these methods are not generally applicable for an arbitrary dose prescription and kernel. Iterative reconstruction methods were developed in the study of imaging science, and their mathematical properties have been well studied. Indeed, these methods can be readily modified and applied to radiotherapy (Xing and Chen 1996). Iterative techniques have many advantages over other approaches. They are conceptually simple to understand, straightforward to implement and many physical effects such as scattering contributions to the absorbed dose and tissue inhomogeneity corrections can be easily incorporated into the algorithms. Furthermore, these techniques can be easily generalized to different beam geometries, and to full three dimensional inverse treatment planning.

The general steps of inverse planning process are:

Step 1: Definition of geometrical parameters of the elemental fluence amplitudes.

Step 2: Selection of beams.

Step 3: Specification of organ parameters.

Step 4: Starting the optimization.

Step 5: Evaluation of achieved results.

The most common clinically used planning and delivery techniques can be divided in 6 categories (Galvin 2006), such as (1) beamlet-based inverse planning combined with either a binary or standard MLC, (2) aperture- based inverse planning with a standard MLC, (3) intensity- modulated arc therapy (IMAT) using a standard MLC, (4) multiple isocenter IMRT using circular collimators, (5) direct aperture optimization using a standard MLC, and (6) IMRT with physical compensators.

\subsection{CONCLUSIONS}

Except for a very few special cases where the optimization problem can be solved analytically (Brahme et al 1982, Cormack 1987, Barth 1990), the optimization process is performed numerically due to the complexity of the problem (Bortfeld et al. 1990, Holmes and Mackie 1994, Webb 1990). However, some analytical formalism is still in use for planning of IMAT and rotational IMRT with concave target volume (Brantengeier 2005, Cotrutz et al. 2000).

The main problem of an analytical solution of the inverse planning is a negative fluence that arises to compensate an excessive irradiation of an OAR or normal tissues. There are some methods to overcome this problem, such as truncation of to zero or addition of a positive constant to the whole intensity profile. The resultant dose distribution will differ, however, from the desired one. 
The inverse planning in radiation therapy is closely related with the $3 \mathrm{D}$ image reconstruction in CT. Thus, the methods employed in image reconstruction can be used to solve the inverse problem in RT. However, it is impossible to incorporate dose constraints or optimization parameters in the algorithms. Therefore, the direct inverse problem is not used currently in the clinical treatment planning.

A common feature of the modern inverse treatment planning is the attempt to minimize (or maximize) a clinical objective function, which expresses the violation of the desired clinical objectives for a specific treatment plan. In general, work in inverse treatment planning can be classified into the following two categories: (1) modeling of the objective function; and (2) methods to search for the minimum (or maximum) of the objective functions. 


\section{ROTATIONAL CONFORMAL RADIOTHERAPY WITH BEAM MODIFYING DEVICES}

\subsection{INTRODUCTION}

In rotational therapy the requirements for tumor uniform dose, minimum dose to the surrounding healthy tissue and protection of Organs at Risk (OARs) are better fulfilled if the beam shape changes continuously during the rotation. This principle is fulfilled in the rotational therapy with gravity-oriented devices (Proimos 1966). The idea of gravity-oriented devices comprises the modulation of the beam intensity by means of absorbers, introduced in the beam and preserving their vertical position by the force of gravity. The protector is attached to a shaft, which is parallel to the axis of rotation, and is kept parallel to itself by means of counterweight attached to the rear end of the shaft. The gravity-oriented protector is a demagnified copy of the OAR and stays "omoiotheta" to the OAR for every gantry position during the rotation, as shown in Fig. 4-1(a). This principle is easily extended to include more than one absorber.

\footnotetext{
1 "Omoiotheta" is a compound word of the Greek words "omoios" that means "same, similar" and "thesi" that means "position". In other words, the beam modulators stay in similar position with the OAR and remain parallel to it during the gantry rotation. For the first time the term was used by Proimos and Goldson (1981).
} 

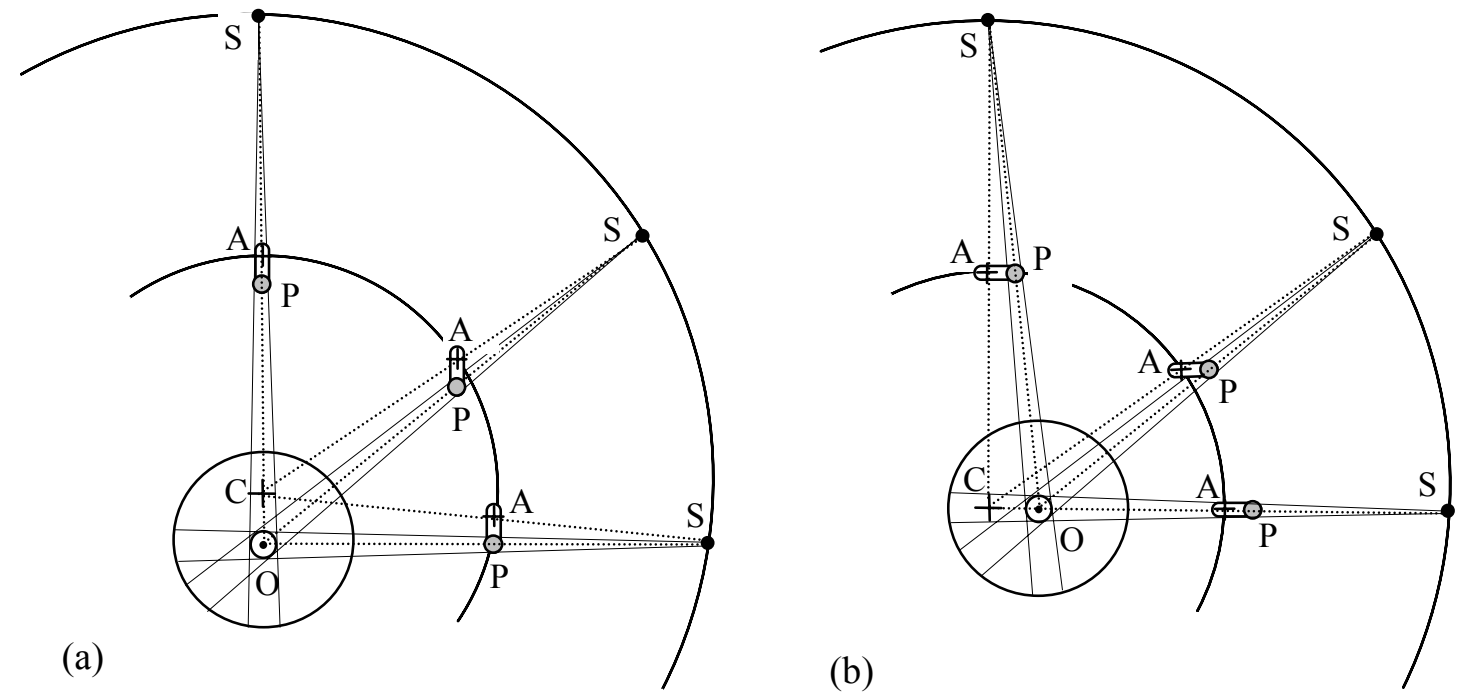

FIGURE 4-1. The gravity-oriented (a) and the "treatment oriented" (b) protectors are shown on the vertical plane of the treatment unit rotation for three positions of the beam. The source is denoted by $\mathrm{S}$, the protector by $\mathrm{P}$, the axis of the shaft of the protector by $\mathrm{A}$, respectively. Point $\mathrm{C}$ is the isocenter and point $\mathrm{O}$ is the center of the OAR. The collimator and beam edges have been omitted for clarity.

The beam sides can be modulated using so-called gravity-oriented shapers (Proimos 1966). Two shapers are attached to two shafts of suspension that are parallel to the axis of gantry rotation. Both shapers keep their direction during rotation due to the gravity.

Although this technique offered good physical results, it was not adopted and applied by the radiotherapeutic community at that time, mainly because the imaging modalities were not developed yet. In addition to this, there were no practical and simple instructions concerning the manufacturing and the use of the gravity oriented absorbers as well as lack of treatment planning programs (Danciu 2001). Since 1997 this technique has been further developed, simplified and standardized (Danciu and Proimos 1997, Danciu and Proimos 1999). In spite of the critical opinion (Webb 2004), this technique has some important advantages (Proimos 1966, Proimos and Goldson 1981, Danciu 2001, Mavroidis et al. 2000): (1) significant protection of the organs and conformal shaping of the field, (2) use of inexpensive and easy deformed materials (like lead, Cerrobend, lead-shot and steel-shot) and (3) there is no need to change the filter for each gantry position, because the OAR is in the shadow of the protector for every gantry angle. Due to these advantages, this technique could be a choice for radiotherapy departments. However, the use of the gravity-oriented devices is accompanied by several inconveniencies, like the manufacturing of the beam modulators, which requires considerable time and efforts, as well as large space that is necessary for their storage. 
In this chapter, studies of beam modifying devices for rotational radiotherapy based on the principles of gravity-oriented devices, but oriented by a motorized system, are presented. An example of movement of such "treatment-oriented" protector is shown in Fig. 4-1(b).

\subsection{PRINCIPLES OF THE BEAM MODIFYING DEVICES}

Intensity modulation with the beam modifying devices is based on the principle of the gravity-oriented devices (Proimos 1961, Proimos 1966, Proimos et al. 1966), shortly described in Chapter 2, sect. 2.5. However, the beam modifying devices are driven by a motorized system and may have any initial orientation in the space, according to the location of the OAR. Subsequently, they preserve their spatial position parallel to the initial one during gantry rotation, as shown in Fig. 4-2. The beam modifying devices are divided in two groups

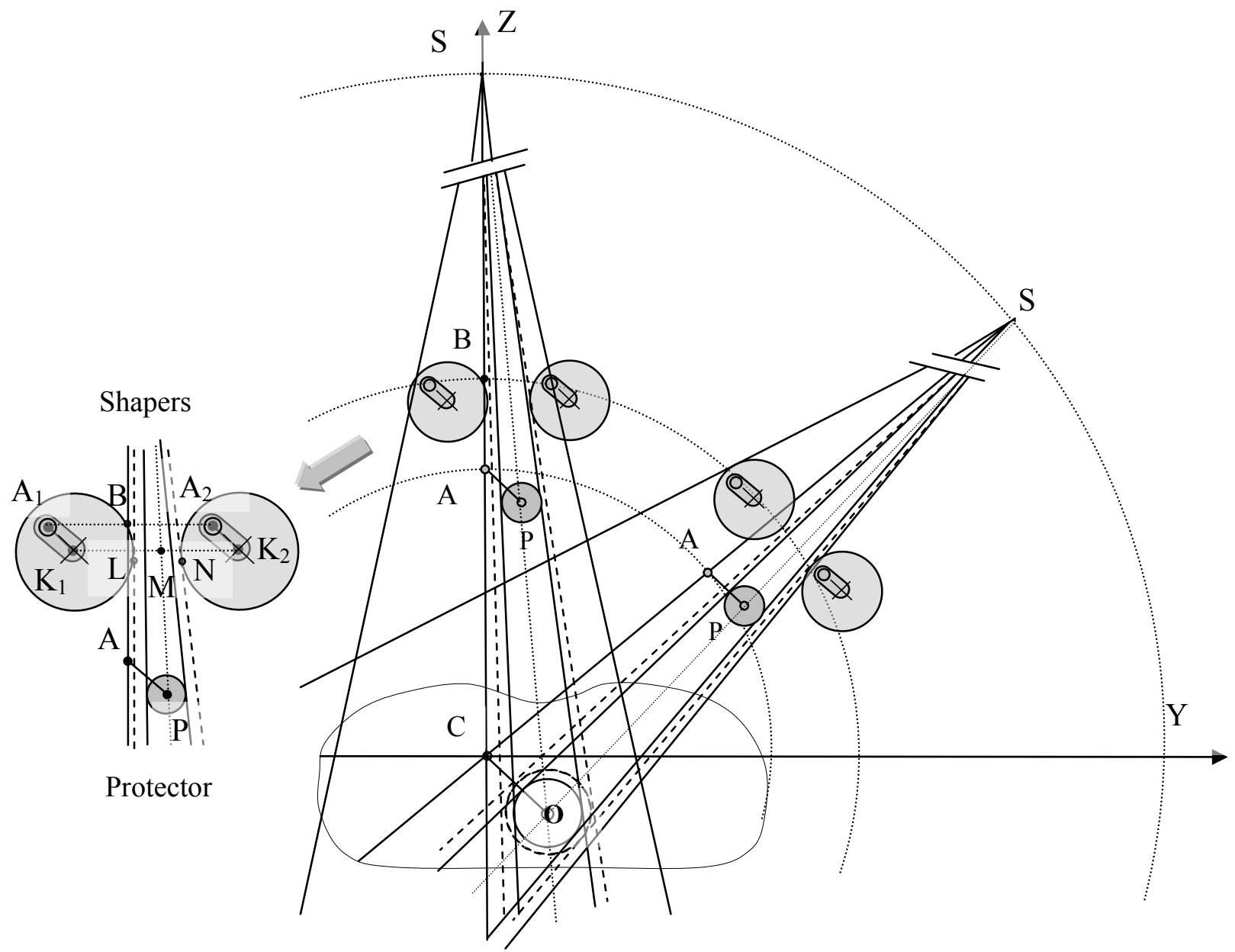

FIGURE 4-2. Beam modifying devices, for rotational radiotherapy including protector and beam shapers. The following labeling is used in this illustration: $S$ is the source, $P$ is the center of the protector, $A$ is the shaft of the protector, $K_{1}$ and $K_{2}$ are the centers of the shapers, $A_{1}$ and $A_{2}$ are the shapers' shafts, $\mathrm{C}$ is the isocenter, $\mathrm{O}$ is the center of the OAR, and LN is the width of the unshaped part of the beam. B and $\mathrm{M}$ are auxiliary points. The collimator has been omitted for clarity. 
in this work, according to their functions: protectors and beam shapers.

Protectors. These are high-density absorbers that significantly reduce the intensity of the beam. Protector defined and used in this work is a copy of the corresponding OAR appropriately scaled according to its distance from the source. Thus, the beam eye's views of the protector and the OAR coincide for every gantry angle. Therefore, during the motion of the gantry, the protector has to be always oriented in such a way in order to preserve parallel position to the OAR, so then the contours of the OAR coincide with the shadow of the protector for every gantry position.

Dose below the protector. The dose under a partial beam attenuator depends upon the amount of primary radiation transmitted through the absorber and the amount of radiation scattered to that point from the phantom. Let us consider the dose at depth $R$ below the surface of the phantom irradiated by a stationary beam of dimensions $W_{0}$, in which an absorber of dimensions $w$ is imposed (both sets of dimensions being defined at depth $R$, i.e. at the isocenter $\mathrm{C}$ ). If the absorber is assumed to transmit a constant fraction $F$ of the incident primary fraction, then the dose will consist of (Rawlingson and Cunningham 1972, El-Khatib et al.1988, Fiorino et al. 1995, Danciu 2001, Khan 1994d):

- a contribution due to primary radiation, given by $\operatorname{TAR}(\mathrm{R}, 0) \cdot \mathrm{F}$, where $\mathrm{TAR}(\mathrm{R}, 0)$ is the zero area tissue-air ratio;

- a contribution of magnitude $\operatorname{SAR}(\mathrm{R}, \mathrm{w}) \cdot \mathrm{F}$ due to scatter from the shielded region, where $\operatorname{SAR}(R, w)$ is the scatter-air ratio for a beam of dimensions $w$;

- a contribution due to scatter from the irradiated region, given by $S A R\left(R, W_{0}\right)$ $\operatorname{SAR}(R, w)$, where $\operatorname{SAR}\left(R, W_{0}\right)$ is the scatter-air ratio for the entire beam.

These terms can be rearranged and expressed in terms of percentage depth doses (PDDs) and backscatter factors (BSFs) for a derivation of an analytical expression for the estimation of the dose under the partial attenuator. The complete derivation is shown in Appendix A.

The dose at depth $R$ delivered by the field $W_{0}$ behind the absorber of width w expressed as a fraction of the dose at the same point in the phantom in the absence of the absorber can be calculated by the following formula:

$$
\frac{\mathrm{D}\left(\mathrm{R}, \mathrm{W}_{0} ; \mathrm{w}\right)}{\mathrm{D}\left(\mathrm{R}, \mathrm{W}_{0}\right)}=1-\frac{\mathrm{BSF}(\mathrm{w})}{\mathrm{BSF}\left(\mathrm{W}_{0}\right)} \frac{\mathrm{PDD}(\mathrm{R}, \mathrm{w})}{\operatorname{PDD}\left(\mathrm{R}, \mathrm{W}_{0}\right)}(1-\mathrm{F})
$$


Although this relationship was derived for a stationary beam, it will apply equally to a moving beam directed towards the center of circular cross-section.

Let us assess the dose reduction at the center of the cylindrical phantom of $15 \mathrm{~cm}$ in diameter (which will be used in our studies later on) provided by a cylindrical protector, made of lead, gold and tungsten of $1.6 \mathrm{~cm}$ in diameter and placed at $68 \mathrm{~cm}$ from the irradiation source. The source-to-isocenter distance (SID) let us assume to be $100 \mathrm{~cm}$ and a field size 10 $\times 10 \mathrm{~cm}^{2}$. The circular protector cross-section of $1.6 \mathrm{~cm}$ can be replaced by the rectangular one of $1.5 \mathrm{~cm}$ (thickness) $\times 1.4 \mathrm{~cm}$ (width), keeping the same cross-sectional area. Thus the width $\mathrm{w}$ of the attenuated field at the isocenter will be $1.4 \mathrm{~cm} \cdot 100 \mathrm{~cm} / 68 \mathrm{~cm}$, that is $2.1 \mathrm{~cm}$.

Using the available values of BSF and PDD (Khan 1994 and data from University hospital of Larissa), the relative dose at the center of the attenuated field $D\left(R, W_{0} ; w\right) / D(R$, $\left.W_{0}\right)=D(7.5,10 ; 2.1) / D(7.5,10)$ was calculated. This ratio of shielded to open beam doses is $0.50,0.35$ and 0.36 for lead, gold and tungsten, respectively. These values may be slightly overestimated, due to the small fields BSF and PDD used in calculations.

Shapers. This is a fact (Ezzell et al. 1987) that an insertion of the attenuator into the treatment beam markedly affects the uniformity of the dose outside the shielded region (see Fig. 4-3a and 4-3b)). A characteristic gradual increase in the dose beyond the protected region is an intrinsic property of the rotational geometry (Rawlingson and Cunningham 1972). Tissues within the Planning Target Volume (PTV), but close to the shielded region, will receive a lower dose than tissues further away, as they remain within the shadow of the protector for a larger fraction of the rotation time. In order to avoid dose increment further away from the protected region, the outer layers of the beam have to be additionally filtered (Rawlingson and Cunningham 1972, Lax and Brahme 1982, Casebow 1990). Such filtering can be provided by cylindrical beam shapers of appropriate material and dimensions (Danciu 2001), as shown in Fig. 4-3(c). The drawback of the additional field shaping, however, is that the dose in the PTV is reduced.

Let us illustrate the magnitude of the dose at the center of the cylindrical phantom, for which both protector and shapers are used. The phantom can represent the neck in the case of a head-and-neck tumor, where the protector is used for a dose reduction to the spinal cord. The treatment doses for head and neck tumors usually are about $70 \mathrm{~Gy}$, whereas the spinal cord dose should not exceed 45 to 50 Gy (Dobbs J. et al. 1999, Martel M.K. et al. 1997, Hunt M.A. et al. 2001, Jena R. et al. 2004, Brantengeier K. et al. 2000, Eisbruch A. et al. 1998, 
Irradiation setup:

(a)

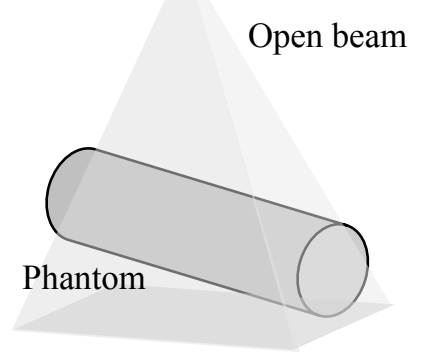

Dose distributions in the central transversal plane of the phantom

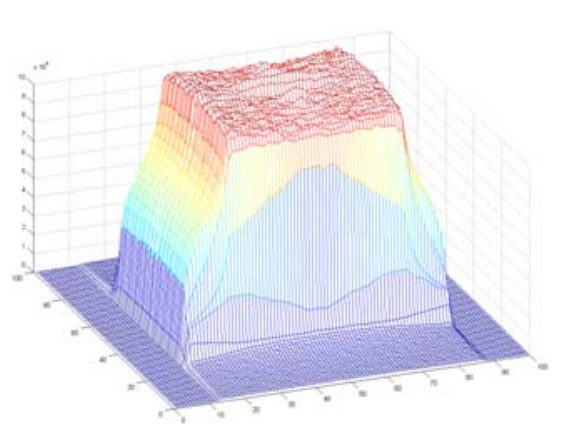

(b)

\section{Protector}
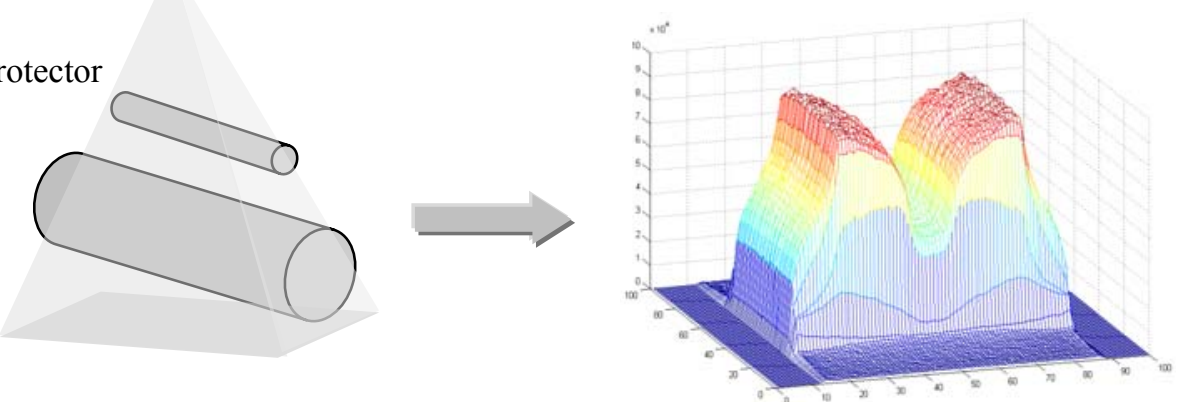

(c)
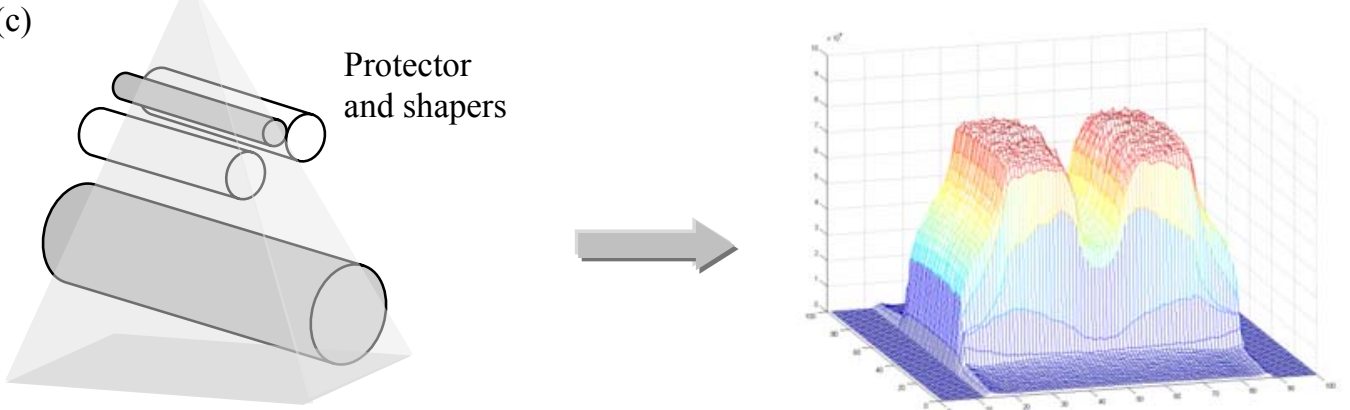

FIGURE 4-3. Phantom irradiation with (a) open beam, (b) with protector inserted in the beam, and (c) with full assembly of beam modifying devices, i.e., protector and shapers and the corresponding dose distributions received after gantry rotation over $360^{\circ}$.

Boyer A.L. et al. 1997). So, the desired dose distribution features have to be as follows: lower than the tolerance dose behind the protector and uniform in the tumor region. Let us choose the dose at the PTV to be, for example, 0.80 of the dose of the open beam, i.e. $D(P T V)=$ $0.80 D\left(R, W_{0}\right)$ due to the use of shapers. Using that $\mathrm{D}\left(\mathrm{R}, \mathrm{W}_{0} ; \mathrm{w}\right) / \mathrm{D}\left(\mathrm{R}, \mathrm{W}_{0}\right)=0.50$ for lead protector of $1.6 \mathrm{~cm}$ in diameter, as presented above, the dose to the spinal cord $\mathrm{D}(\mathrm{SC})=$ $\mathrm{D}\left(\mathrm{R}, \mathrm{W}_{0} ; \mathrm{w}\right)=0.50 \mathrm{D}\left(\mathrm{R}, \mathrm{W}_{0}\right)$ or $\mathrm{D}(\mathrm{SC})=0.50[\mathrm{D}(\mathrm{PTV}) / 0.80]$, from where the value of the dose at the spinal cord can be determined and is $43.8 \mathrm{~Gy}$ if $\mathrm{D}(\mathrm{PTV})=70 \mathrm{~Gy}$ is assumed. For gold and tungsten, for which the ratio $\mathrm{D}\left(\mathrm{R}, \mathrm{W}_{0} ; \mathrm{w}\right) / \mathrm{D}\left(\mathrm{R}, \mathrm{W}_{0}\right)$ is even lower $(0.35$ and 0.36 , respectively) the dose at the spinal cord will be $30.6 \mathrm{~Gy}$ and $31.5 \mathrm{~Gy}$. The values of the 
achieved beam attenuation at the center of the OAR are evidence of a sufficient OAR protection.

\subsection{MATHEMATICAL MODELING OF THE BEAM MODIFYING DEVICES}

\subsubsection{Protectors}

A protector is a miniaturized copy of the OAR that keeps the OAR in its shadow for every gantry position (Proimos 1963, Proimos 1966). The protector P (see Fig. 4-2) is attached to a shaft $\mathrm{A}$, which is on the central ray and rotates synchronously with the source $\mathrm{S}$ around the isocenter $\mathrm{C}$. At the same time, the protector stays in a similar position to the OAR, while the line segment AP, connecting the shaft A with the center of the protector $\mathrm{P}$, remains parallel to the line segment CO during rotation. The linear dimensions of the protector can be calculated from the similar triangles. The triangle SPE is similar to the triangle SOE' (see Fig. 4-4(a)), so

$$
\frac{\mathrm{PE}}{\mathrm{OE}^{\prime}}=\frac{\mathrm{SP}}{\mathrm{SO}}
$$

where $P E=R_{p r}$ is the radius of the protector and $\mathrm{OE}^{\prime}=\mathrm{R}_{\mathrm{OAR}}$ is the radius of the OAR.

Moreover, the triangles SPA and SOC are similar, therefore:

$$
\frac{\mathrm{SP}}{\mathrm{SO}}=\frac{\mathrm{SA}}{\mathrm{SC}}
$$

Hence, combining two equations, we get:

$$
\frac{\mathrm{R}_{\mathrm{pr}}}{\mathrm{R}_{\mathrm{OAR}}}=\frac{\mathrm{SA}}{\mathrm{SC}} .
$$

By introducing a scaling factor $S F$, which is the ratio of the source-to-protector's shaft distance SA to the source-to-isocenter distance SC, the radius of the protector can be determined as $\mathrm{R}_{\mathrm{pr}}=\mathrm{R}_{\mathrm{OAR}} \cdot S F$. For a general case, any linear dimension of the protector is $S F$ times the corresponding dimension of the OAR. The length of the cylindrical protector $\mathrm{L}_{\mathrm{pr}}$ is correspondingly $\mathrm{L}_{\mathrm{pr}}=\mathrm{L}_{\mathrm{OAR}} \cdot S F$, where $\mathrm{L}_{\mathrm{OAR}}$ is the length of the cylindrical OAR.

The initial coordinates of the protector $\left(\mathrm{x}_{0 \mathrm{pr}}, \mathrm{y}_{0 \mathrm{pr}}, \mathrm{z}_{0 \mathrm{pr}}\right)$ are derived using a similarity of the triangles APP' and COO' (see Fig. 4(b)) and can be determined, as following:

$$
\mathbf{r}_{\mathbf{0 p r}}=\mathbf{r}_{\mathbf{0 A}}+\mathrm{SF} \cdot \mathbf{r}_{\mathrm{OAR}}
$$



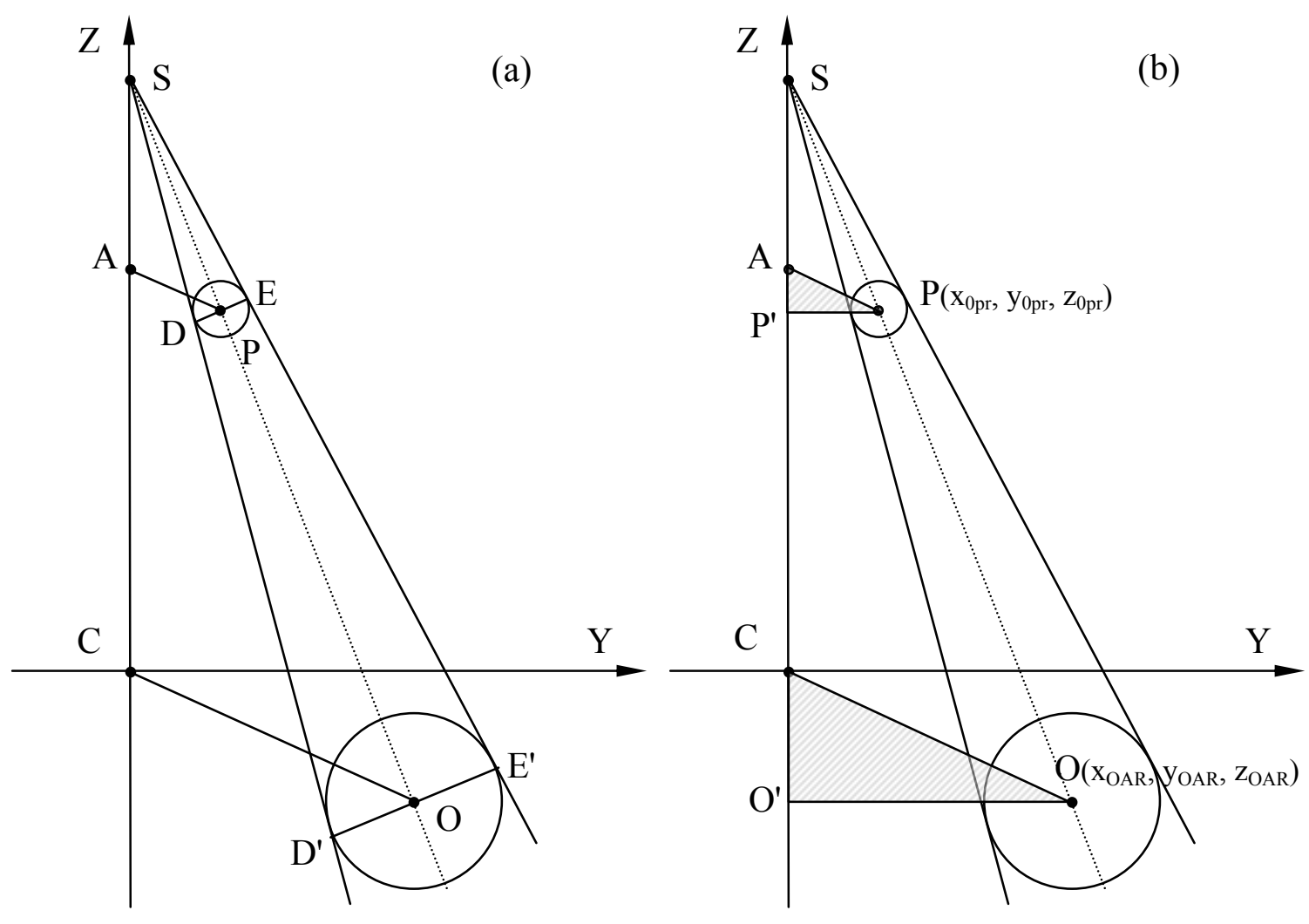

FIGURE 4-4. Schematic representation of the auxiliary geometrical constructions for the calculation of the protector's diameter (a) and protector's coordinates (b).

where $\mathbf{r}_{\mathbf{0 p r}}=\left(\mathrm{x}_{0 \mathrm{pr}}, \mathrm{y}_{0 \mathrm{pr}}, \mathrm{z}_{0 \mathrm{pr}}\right)^{\mathrm{T}}$ is the position vector of the initial coordinates of the protector (at $0^{\circ}$ gantry angle), $\mathbf{r}_{\mathbf{0 A}}$ is the vector of the initial position of the shaft $\mathrm{A}$ and $\mathbf{r}_{\mathbf{O A R}}$ is the position vector of the center of the OAR. The initial position of the protector's shaft depends on the attachment of the protector to the accelerator head.

The position vector $\mathbf{r}_{\mathbf{p r}}=\left(\mathrm{x}_{\mathrm{pr}}, \mathrm{y}_{\mathrm{pr}}, \mathrm{z}_{\mathrm{pr}}\right)^{\mathrm{T}}$ of the protector's center during gantry rotation for any gantry angle $\theta$ is given by the following equation:

$$
\mathbf{r}_{\mathbf{p r}}=\mathrm{R}_{\mathrm{x}} \cdot \mathbf{r}_{\mathbf{0 A}}+\mathrm{SF} \cdot \mathbf{r}_{\mathrm{OAR}}
$$

where

$$
\mathrm{R}_{\mathrm{x}}=\left(\begin{array}{ccc}
1 & 0 & 0 \\
0 & \cos \theta & \sin \theta \\
0 & -\sin \theta & \cos \theta
\end{array}\right)
$$

is the matrix describing the rotation around the $\mathrm{x}$ axis.

\subsubsection{Beam shapers}

The shapers are suspended by the shafts $A_{1}$ and $A_{2}$, which are parallel to the axis of rotation $\mathrm{C}$ and rotate synchronously with the source $\mathrm{S}$, as shown in Fig. 4-2. Each of the 
shapers remains parallel to its initial orientation during gantry rotation; i.e., lines $\mathrm{A}_{1} \mathrm{~K}_{1}$ and $\mathrm{A}_{2} \mathrm{~K}_{2}$ stay parallel to themselves (see more detailed illustration in Fig. 4-5).

The coordinates of the centers of the shapers for any gantry rotation angle are calculated by considering three main features of the shapers motion:

(1) The centers $K_{1}$ and $K_{2}$ of the shapers are located symmetrically to the ray SO, going through the center of an OAR, i.e., $\mathrm{K}_{1} \mathrm{M}=\mathrm{MK}_{2}$;

(2) The shafts $A_{1}$ and $A_{2}$ are placed symmetrically regarding to the central ray SC of the beam, i.e., $\mathrm{A}_{1} \mathrm{~B}=\mathrm{BA}_{2}$;

(3) The line $A_{1} A_{2}$ is parallel to $K_{1} K_{2}$ and both are perpendicular to the central ray SC of the beam, i.e., $A_{1} A_{2} \| K_{1} K_{2}$ and $A_{1} A_{2} \perp S C$.

Thus, the beam modifying devices perform both rotational and translational motion in order to change the beam shape continuously during gantry rotation. The vectors of the centers $\mathrm{K}_{1}$ and $\mathrm{K}_{2}$ of the shapers during the gantry rotation are correspondingly:

$$
\mathbf{r}_{\text {Sh1 }}=\mathrm{R}_{\mathrm{x}} \cdot \mathbf{r}_{\mathbf{0 A 1}}+\alpha \cdot \mathbf{r}_{\mathbf{O A R}}
$$

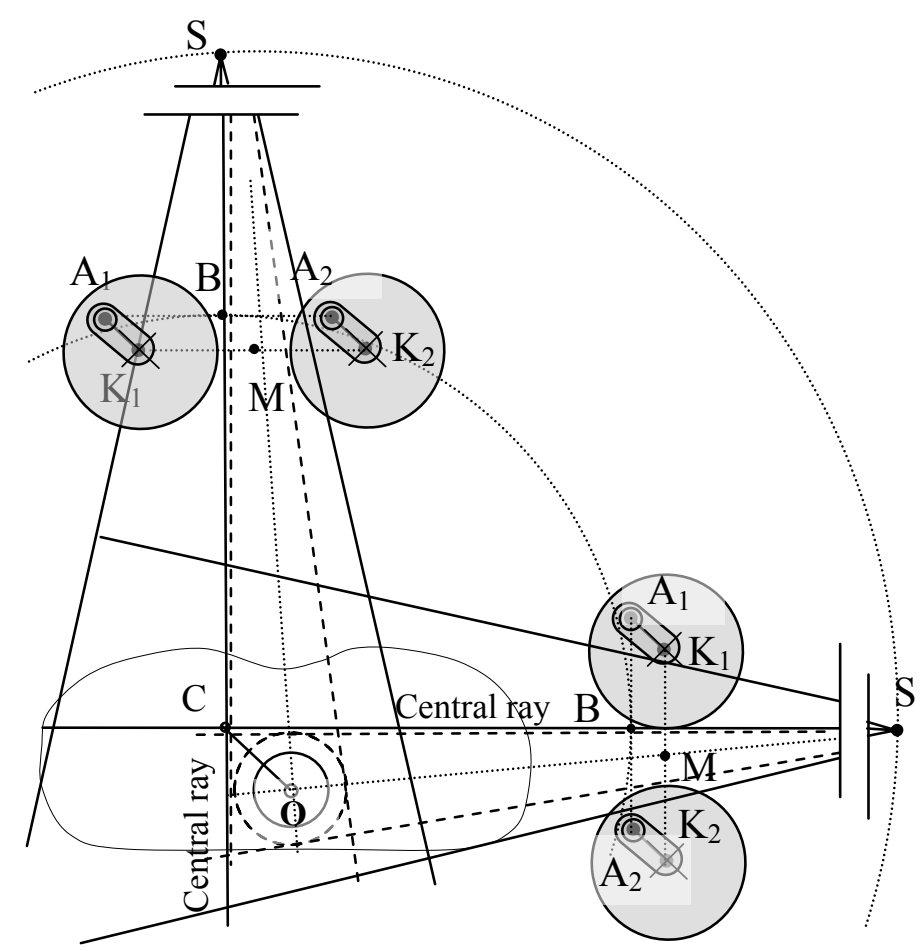

FIGURE 4-5. Illustration of the movement of the beam shapers during gantry rotation. The labeling used: $\mathrm{S}$ is the source, $\mathrm{K}_{1}$ and $\mathrm{K}_{2}$ are the centers of the shapers, $\mathrm{M}$ is the middle point between $K_{1} K_{2}, A_{1}$ and $A_{2}$ are the shapers' shafts, $B$ is the middle point between $A_{1} A_{2}, C$ is the isocenter and $\mathrm{O}$ is the center of the OAR. 
and

$$
\mathbf{r}_{\text {sh2 }}=\mathrm{R}_{\mathrm{x}} \cdot \mathbf{r}_{\mathbf{O A 2} 2}+\alpha \cdot \mathbf{r}_{\mathbf{O A R}}
$$

where $\alpha$ is the ratio of the source-to-shaper's shaft distance SB to the source-to-isocenter distance SC and $\mathbf{r}_{\mathbf{0 A} 1}$ and $\mathbf{r}_{\mathbf{0 A 2}}$ are the vectors of the initial position (at $0^{\circ}$ gantry angle) of the shafts $A_{1}$ and $A_{2}$. These vectors are determined by an arrangement of the attachment of the beam shapers to the linac head. The y-coordinates of the shapers' shafts initial position are $\pm \mathrm{A}_{1} \mathrm{~A}_{2} / 2$.

As the dose outside the OAR increases nonuniformly, the beam must be increasingly filtered as the distance from the center of the OAR increases, as was mentioned above, as was mentioned above. Circular shapers can be suitable for this purpose, because an attenuating chord of such shapers increases non-linearly as well, as the beam passes further from the edge of the shaper.

There are two approaches for the determination of the shapers' parameters. The first: "from diameter to material", that is to fix the diameter of the protector, and afterwards to determine the material. Another approach "from material to diameter" is to define the material of the shapers and the desired dose decrease at a chosen point with consequent calculations of the geometrical parameters of the shapers.

\subsubsection{1st method of shapers' parameters calculations: "From diameter to material"}

Figure 4-6 summarizes schematically the first method of calculations of the dimensions and the material of the beam shapers (Danciu 2001).

The calculations of the shapers' parameters begin with a definition of the margins, from where the beam attenuated by shapers will start. This information can be extracted from the dose profile for a single protector, calculated or experimentally measured. A decision, where shapers' inner edges ( $\mathrm{L}$ and N, according to Fig. 4-7) will be placed, depends on the distance at what PTV starts or/and on the desirable dose increase just outside the shielded region. The distance L'N', beyond which the beam will be filtered by shapers, will be called here the dose build-up region. The unfiltered beam width LN can be found from the dose build-up region, projecting it to the level of shapers, as is shown in Fig. 4-7. The shapers' diameter is calculated by subtracting $L N$ from the distance $K_{1} K_{2}$, which is the distance between the centers of the shapers. 


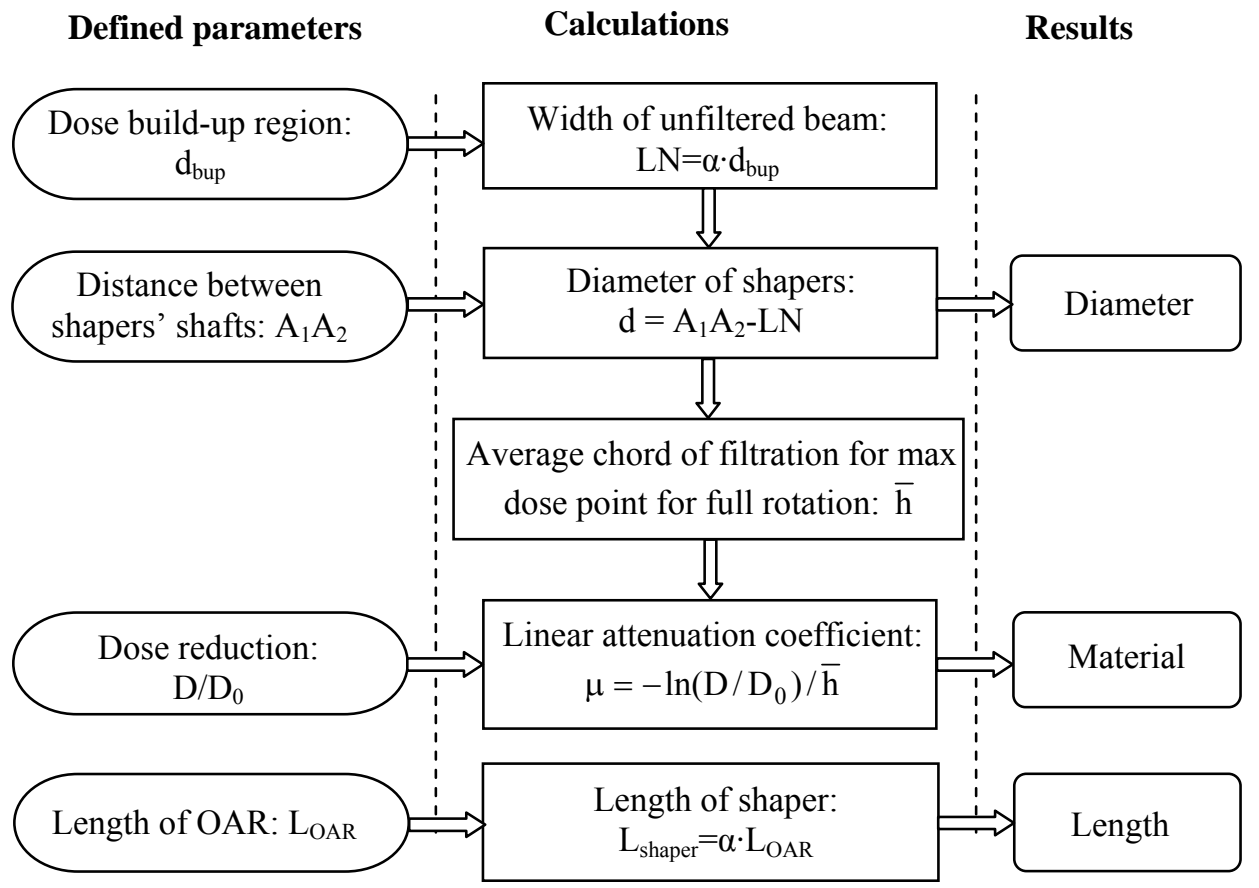

FIGURE 4-6. A schematic representation of the shapers' parameters calculation method "from diameter to material". Here, alpha is a ratio of the source-to-shaper's shaft distance to the source to isocenter distance $(\mathrm{SB} / \mathrm{SC})$.
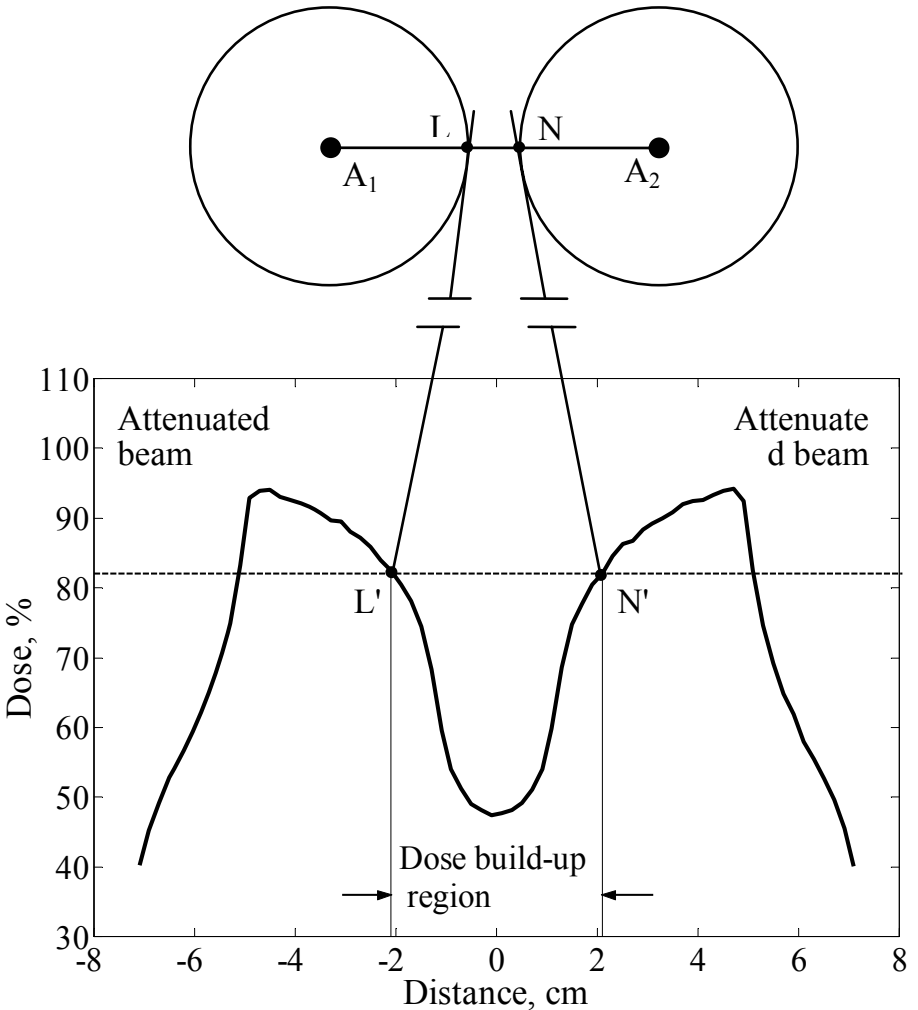

FIGURE 4-7. Explanatory scheme for shapers' location calculations.
Due to the complicated motion of the shapers during the gantry rotation, a given point of the phantom will be under different shaper's filtrations for every gantry angle, i.e., the same ray will pass different paths in a shaper's material. Therefore, for simplicity the average filtration chord was calculated, as in a case in which a given point is assumed to be under the constant filtration for every angle:

$$
\overline{\mathrm{h}}=\frac{\sum_{\mathrm{i}=1}^{\mathrm{n}} \mathrm{h}_{\mathrm{i}}}{\mathrm{n}},
$$

where $\mathrm{n}$ is the number of gantry 
angles used for calculations. The method of calculation of a filtration chord is presented in appendix B.

The linear attenuation coefficient was calculated by:

$$
\mu=\frac{\ln \frac{D}{D_{0}}}{\bar{h}},
$$

where $\mathrm{D} / \mathrm{D}_{0}$ is the desired dose reduction (see Fig. 4-6). The length of the shaper is $\alpha$ times the length of the corresponding OAR. Summarizing, the parameters used as input data for the calculations of the physical (material) and geometrical (length and diameter) parameters of the beam shapers are: the dose build-up region, the distance between the shapers' shafts, the desired intensity attenuation, and the length of the OAR.

The described method has direct solution, however, its disadvantage is a limited number of materials, suitable for shapers design for a given geometrical parameters. Therefore, some preceding experience or information can help in choosing the protector's diameter, leading to the proper material.

\subsubsection{2nd method of shapers' parameters calculations: "From material to diameter"}

This approach doesn't have direct solution, thus the shapers' diameter is determined iteratively. Figure 4-8 generalizes the method of calculations of the geometrical parameters of the shapers made of a given material. The dose built-up region and the width of the unfiltered beam are determined as described in the previous method (sect. 3.3.2.1). Calculations of the average chord of filtration for a maximal dose point involve both shaper's diameter and the distance between the edges of the shapers LN (i.e. the unfiltered beam width). By defining the arbitrary initial shapers' diameter and calculating the average attenuating chord length, it can be compared with the desired attenuating chord length, which is calculated based on the desired dose change and weighted mean linear attenuation coefficient:

$$
\overline{\mathrm{h}}=\frac{\ln \frac{\mathrm{D}}{\mathrm{D}_{0}}}{\bar{\mu}} .
$$




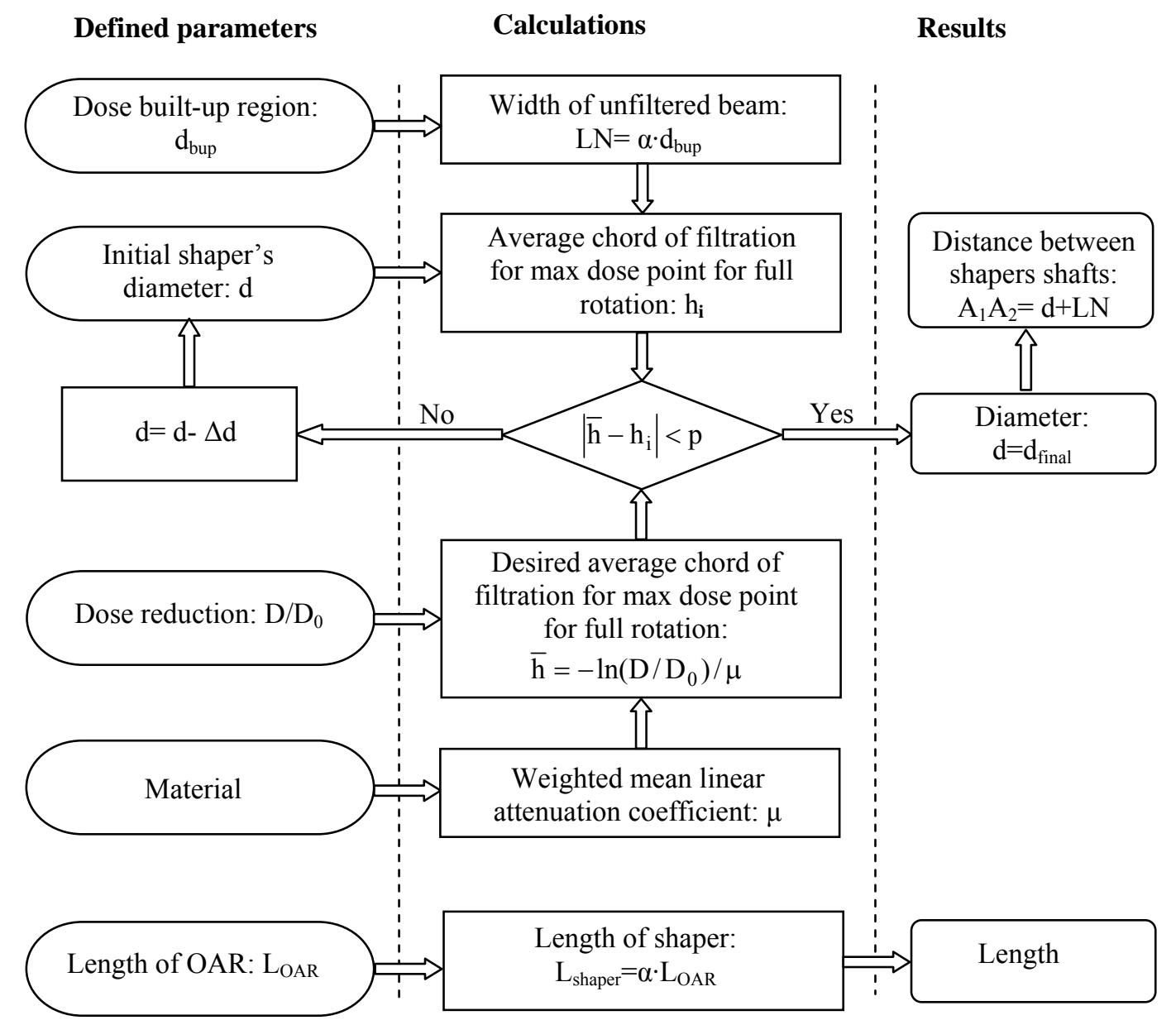

FIGURE 4-8. A schematic representation of the shapers' parameters calculation method "from material to diameter". Here, alpha is a ratio of the source-to-shaper's shaft distance to the source to isocenter distance $(\mathrm{SB} / \mathrm{SC}), \Delta \mathrm{d}$ is the predefined lessening of the diameter per iteration and $\mathrm{p}$ is the parameter that defines the maximal allowed difference of the calculated average chord value and one resulted from the features of the desired dose distribution.

If the difference is bigger that $\mathrm{p}$, defined a priory, the diameter is decreased by $\Delta \mathrm{d}$ and the calculations are repeated. The weighted-mean linear attenuation coefficient $\mu$ was calculated according to (Levy at al. 1974):

$$
\bar{\mu}=\frac{\int_{0}^{E_{\max }} \Psi(E) \cdot \mu(E) d E}{\int_{0}^{E \max } \Psi(E) d E},
$$

where $\Psi(E) d E$ is energy fluence for photons with energy between $E$ and $E+d E$ and $\mu(E)$ is linear attenuation coefficient for the energy E. For a small energy interval $\mathrm{dE}$, the energy fluence can be considered $\Psi(\mathrm{E}) \mathrm{dE}=\mathrm{E} \cdot \mathrm{N}(\mathrm{E}) \mathrm{dE}$, so 


$$
\bar{\mu}=\frac{\int_{0}^{E_{\max }} E \cdot N(E) \cdot \mu(E) d E}{\int_{0}^{E \max } E \cdot N(E) d E} .
$$

The shaper's diameter that satisfies the inequality $\left|\overline{\mathrm{h}}-\mathrm{h}_{\mathrm{i}}\right| \leq \mathrm{p}$ corresponds to the final shapers' diameter and the distance between the shafts of the shapers is calculated: $A_{1} A_{2}=d+$ LN. The length of the shaper is $\alpha$ times the length of the corresponding OAR, where $\alpha$ is the ratio of the source-to-shaper's shaft distance SB to the source-to-isocenter distance SC.

\subsection{DISCUSSION AND CONCLUSIONS}

The beam modifying devices, presented in this chapter, were divided in two groups: protectors and beam shapers. The principles of their operation are based on the principles of the gravity-oriented devices and resulting beam shaping is similar to that one provided by the gravity-oriented devices, but their motion is motor-driven. During the gantry rotation a protector rotates so, that its cast shadow coincides with the OAR at any gantry position, thus significantly reducing the primary radiation. The principle how to model the protector has already been contained in the idea of the protection itself. The protector is a diminished copy of the OAR that is scaled accordingly to its distance from the irradiation source. The protector is translated during the gantry rotation to be "omoiotheta" to the OAR. This approach primarily is based on the geometric considerations. The important question to be answered is if a protector of a given diameter provides an adequate protection. Also, a location of the PTV and, thus, the desired dose increase at the borders of the protected area is of importance. A dose distribution is a function of many parameters, such as beam energy, field size, materials of the protector and phantom and their relative position. The effect of the protector's material, location and diameter on the dose distribution was studied and results are presented and discussed in Chapter 6. Brahme (1988) pointed out that, in fact, with high energy beams or more specifically when the influence of beam attenuation and scatter is negligible, the optimal incident beam approaches a true geometric projection of the shape of the target volume. When low megavoltage beams are used, the considerable influence of photon attenuation and scatter in this case will make the optimal incident beams to deviate substantially from a pure geometrical projection of the target volume. A protector, in order to attenuate substantially the 
beam, has to be made of a high density material, as the Compton effect is predominant for clinically used x-ray beams. Dancui (2001), discussing the criteria for the choice of the protector's material, proposed to be based on the exponential $\mu \mathrm{x} \approx 1$, which means that $\mathrm{I} / \mathrm{I}_{0}=$ $\exp (-1)$, i.e., $\left(\mathrm{I} / \mathrm{I}_{0}\right) \cdot 100 \% \approx 37 \%$. If scatter is added, beam attenuation will not exceed $60 \%$ that could be enough for protection of most critical organs.

The other constituents of the beam modifying devices are beam shapers. Their task is to filter an excessive irradiation leading to a nonuniform dose outside the OAR and, thus, to ensure a uniform dose at the PTV. The beam shapers are located at both sides of the protector, leaving the circular annulus around the OAR for a dose build-up. This principle of increasing the incident fluence immediately beyond the shielded region appears also in the analytical solution of the direct inverse problem in rotational radiotherapy (Brahme et al. 1982). Ideally, this increase has to be infinitely large, in reality some finite increase is sufficient (Lax and Brahme 1982). Two methods of beam shapers calculations were presented. The use of the first one leads to a proper material for the shapers of a defined diameter, and the second method, conversely, searches for an optimal shaper's diameter of a given material. They are compared and evaluated in the next chapter. The choice of the materials of the shapers was based on the fact that the maximal dose reduction is about $20 \%$. So, low density material can be preferred. This is due to the fact that the transmission through low density materials is not very sensitive to small inaccuracies in a thickness due to the calculation method or introduced during manufacturing process. Additionally, due to a circular symmetry of the rotational treatment and complicated character of shapers' motion, the shapers should be of a circular shape. Thus, the lateral axis of the shapers, that depends on the extension of the irradiation field and should cover the whole non-uniform dose volume, which is quite large, should be equal to the shaper's attenuating thickness in the perpendicular direction. So, the shapers can not be made of high density material.

The motion and calculations of the position and parameters for every piece of the beam modifying devices were described mathematically, preparing, in such a way, the background for a software development for automatic calculations of the devices for a certain clinical situation. Further on, the modeled beam modifying devices can be involved in RT treatment simulations for successive studies and analysis of the achieved dose distributions. 


\section{SIMULATIONS STUDIES ON USE OF THE BEAM MODIFYING DEVICES FOR SPINAL CORD PROTECTION}

\subsection{INTRODUCTION}

Tumors in the regions located near the spinal cord present a treatment challenge to oncologists (Jena R. et al. 2004). In order to achieve tumor control, high doses must be delivered to the target volume, while ensuring that the dose to the spinal cord is kept below tolerance. The spinal cord is the part of the central nervous system that is enclosed in and protected by the vertebral column. The normal ranges of the cord width are $(1.62 \pm 0.12) \mathrm{cm}$ for a cervical spine, $(1.38 \pm 0.39) \mathrm{cm}$ and $(1.94 \pm 0.38)$ for a thoracic and a lumbar spine, respectively. The thickness in an anterior-posterior direction is $(0.98 \pm 0.16) \mathrm{cm},(1.07 \pm 0.30)$ $\mathrm{cm}$ and $(1.60 \pm 0.28) \mathrm{cm}$ for cervical, thoracic and lumbar spine, correspondingly (Mota H.C. et al. 1988).

Such head and neck tumors, like tumor of larynx, hypopharynx, thyroid, and others located near the spine offer, however, a region free of disease around the spinal chord for dose build-up, because the vertebra is usually free of a disease. This is a good prerequisite for the use of beam modifying devices, described in Chapter 4, in rotational mode, because of a gradual increase of the dose beyond the protected region, which is characteristic for a rotational geometry (Rawlingson and Cunningham 1972). Additionally, the high dose closer 
conforms to more or less spherical tumor volume than in the conventional treatment techniques, where the treatment is delivered using four orthogonal beam directions (Brahme 1988).

Simulation studies of field shaping in rotational therapy by means of beam modifying devices were addressed to head and neck tumors in this chapter. Conformation of the dose distribution to treatment geometry has to be achieved as a result of the irradiation, i.e., an adequate protection of the spinal cord as well as a uniform dose at the PTV have to be provided. The spinal cord becomes an OAR in this case.

The classical radiotherapy of head and neck cancers employs parallel opposed lateral photon fields for a significant portion of treatment. The spinal cord is blocked after 40-45 Gy and, so, is excluded from further direct irradiation. With curative intent doses in the range of 70 Gy are needed, whereas the spinal cord dose should not exceed 45 to 50 Gy (Dobbs J. et al. 1999, Martel M.K. et al. 1997, Hunt M.A. et al. 2001, Jena R. et al. 2004, Brantengeier K. et al. 2000, Eisbruch A. et al. 1998, Boyer A.L. et al. 1997).

The objectives of the work presented in this chapter were: (1) to develop a software module for calculation of the parameters and initial coordinates of the beam modifying devices; (2) to carry out simulation studies of field shaping by different configurations of the beam modifying devices, using solid-geometry and voxel-based phantoms, and to evaluate the degree of protection of OAR and the quality of dose distribution outside the protected region, in terms of uniformity; (3) to verify the methods of shapers calculations, presented in sect. 4.3.2. The studies were performed for a case in which the target volume to be irradiated is near the spinal cord, which is to be protected.

\subsection{METHODS AND MATERIALS}

\subsubsection{The Monte Carlo simulator}

Although the physics of photon and electron interactions in matter is well understood, in general it is impossible to develop an analytic expression to describe particle transport in a medium. One widely used technique for solving this problem involves Monte Carlo simulation of radiation transport, in which one uses knowledge of the probability distributions governing the individual interactions of electrons and photons in materials to simulate the 
random trajectories or histories of individual particles (Rogers 2002). One keeps track of physical quantities of interest for a large number of such histories to provide information about required quantities and their distributions.

Studies of field shaping in this work were carried out using the in-house developed Monte Carlo Radiation Therapy Simulator (MCRTS) (Bliznakova et al. 2004), which simulates the radiation transport through a user-defined system, including source, collimators, shielding absorbers, phantom, and detector. The MCRTS is comprised of the following main modules: (1) phantom modeling, (2) High Energy Simulation Module (HESM), (3) image formation modeling and (4) visualization facilities. Phantoms are modeled as a synthesis of geometrical and/or voxelized primitives. The former are simple uniform geometrical shapes such as spheres and cylinders with user-defined dimensions, location, orientation, and composition. In voxelized primitives, the volume of interest is divided into voxels and is represented by a three-dimensional matrix carrying the information of the content of each voxel. The HESM performs four tasks: (a) geometry modeling, able to simulate isocentric rotational geometries. Parameters of the simulated geometry include the distance from the source to the center of rotation, the gantry angle arc, and the step; (b) beam modeling, characterized by the particle charge, energy, direction, position, and the source size considered either as a point source or as having an effective size; (c) dose matrix definition- a three dimensional dose matrix used to calculate the energy deposited by electrons and photons; and (d) irradiation transport modeling of all photon and electron interaction processes with matter in the radiotherapy energy range. The simulation starts with a particle (photon/electron) selected from the initial beam distribution and defined by its energy and angle. The MC code is then used to transport this particle through the medium. In the case of a photon, it is traced until it leaves the defined geometry or its energy drops below the photon cut-off energies $\left(\mathrm{P}_{\text {cut }}\right)$ defined by the user. The adopted model of the physics includes four types of interactions for photons: photoelectric absorption, Compton scattering (including electron binding effects), coherent scattering and pair production. The model of electrons includes bremstahlung production, Moller scattering, and multiple elastic scatterings. Highspeed electrons, produced by the photoelectric absorption, incoherent scattering or pair production undergo multiple scattering through the phantom until their eventual absorption. Electrons with energies greater than certain energy thresholds participate in the production of bremsstrahlung and Moller discrete interactions. The image formation module generates projection images representing two-dimensional spatial distributions of the energy absorbed 
in the detector. Finally, in the 3D Visualization module the designed system, subjected to simulation, is visualized.

At the root of the Monte Carlo method is a simple computational structure where a process is modeled by producing a large number of possible outcomes. For $\mathrm{N}$ events, the mean and variance of a simulation are calculated as

$$
\mu=\frac{1}{\mathrm{~N}} \sum_{\mathrm{i}=1}^{\mathrm{N}} \mathrm{x}_{\mathrm{i}}
$$

and

$$
\sigma^{2}=\frac{1}{\mathrm{~N}-1} \sum_{\mathrm{i}=1}^{\mathrm{N}}\left(\mathrm{x}_{\mathrm{i}}-\mu\right)^{2}
$$

where $\mu$ is the mean, $\sigma^{2}$ is the variance and $x_{i}$ is considered an independent event. The statistical uncertainty on the mean is calculated such that

$$
\delta=\frac{\sigma}{\sqrt{\mathrm{N}}}
$$

where one can see that the accuracy of a Monte Carlo simulation is proportional to $1 / \sqrt{\mathrm{N}}$.

\subsubsection{The software tool for calculations of beam modifying devices}

In order to facilitate the computations of the geometrical setup of the beam modifying devices, a software tool was developed and integrated into the above program. The interface of this tool (Fig. 5-1) enables the input of the geometrical setup and the selection (from the list of objects) of the protected organ or area at risk. The software tool calculates the location of the center and the dimensions of the beam modifying devices, based on information about the shape and the size of the OAR using mathematical background described in details in Chapter 4, sect. 4.3, and updates the files describing the geometry during the simulation.

The software application has been written and compiled using Borland $\mathrm{C}++$ Builder 5.0 just as the MCRTS. 


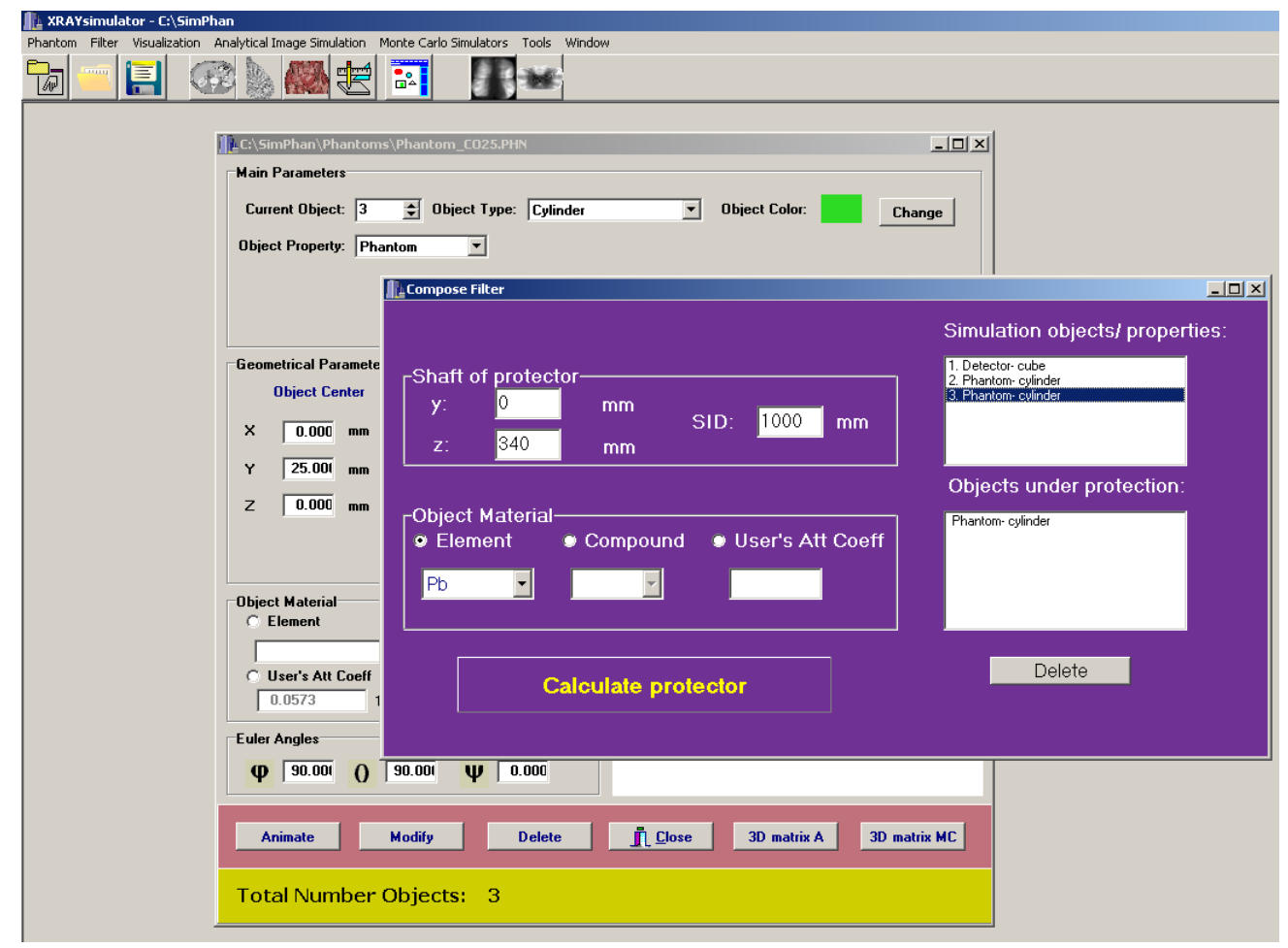

FIGURE 5-1. A screen shot of the software tool for computations of the parameters and coordinates of beam modifying devices for radiotherapy simulations.

\subsubsection{The spectra of irradiation used}

In our simulations monoenergetic and polyenergetic energy fluences were involved.

A $6 \mathrm{MV}$ beam was used with two different spectral distributions: the original spectral distribution of the ELECTA SL75-5 machine, provided by Dr. G. Malalara (University Hospital of Patras, Greece) and spectrum of the ELECTA SL75-5 beam, hardened by a slab of lead $1.4 \mathrm{~cm}$ in thickness, calculated from the initial by MC simulations, which was introduced in order to be able to compare the simulation results with the experimental data, acquired at such conditions. Both spectra are presented in Fig. 5-2.

A $2 \mathrm{MeV}$ monoenergetic beam was also exploited in some simulations of verification studies.

\subsubsection{Neck models and irradiation setups}

A case for protection of the spinal cord during irradiation of a region located near or around it was chosen for our simulation studies. The solid-geometry (mathematical) and the 


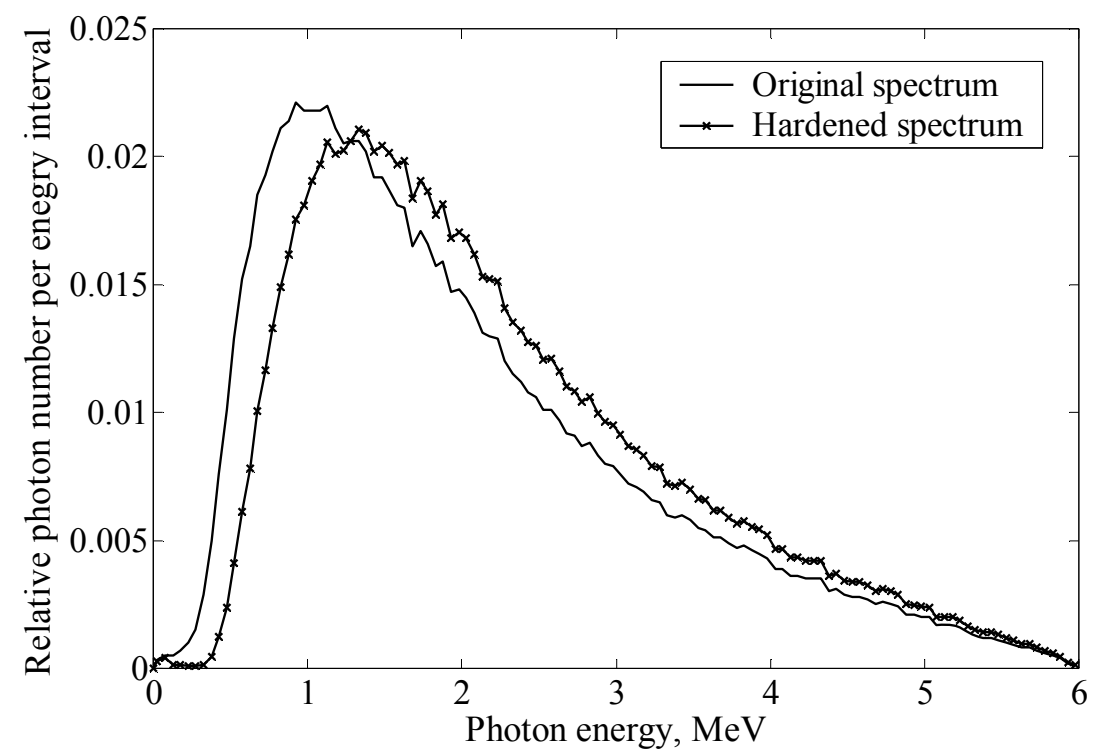

FIGURE 5-2. Original spectrum of the ELECTA SL75-5 linear accelerator and spectral distribution of the beam, hardened with a slab of the lead $1.4 \mathrm{~cm}$ in thickness. The photon number per energy interval was normalized to the total number of photons.

voxel-based (tomographic) models that were used in the simulations are briefly described below.

\subsubsection{Solid-geometry neck model}

The solid-geometry neck model was composed of a cylindrical object with another cylinder of smaller diameter inserted in it, imitating the "spinal cord". The OAR, with its center designated by $\mathrm{O}$, was located in the center of the neck model, as shown in Fig. 5-3. Right-handed Cartesian coordinates were used in our experiments with a reference point coinciding with the isocenter $\mathrm{C}$. The $\mathrm{X}$ axis was parallel to the axes of the both cylinders. The isocenter was placed at one of the points along the phantom's diameter at the central plane of the neck phantom (XY plane), depending on the experimental setup. Two lateral arrangements of the isocenter were used in the case of the solid-geometry model investigations. In the first one, the isocenter coincided with the center of the OAR (Fig. 5-3, if $\mathrm{CO}=0 \mathrm{~cm}$ ), whereas in the second arrangement the isocenter was shifted $2.5 \mathrm{~cm}$ relatively to the center of the OAR along the $y$ axis $(\mathrm{CO}=2.5 \mathrm{~cm})$. The cylindrical neck model of $15 \mathrm{~cm}$ in diameter and of unit density was irradiated by a photon beam, collimated to a $10 \times 10 \mathrm{~cm}^{2}$ field size at the isocenter, in all studies of this chapter involving solid-geometry phantoms. 


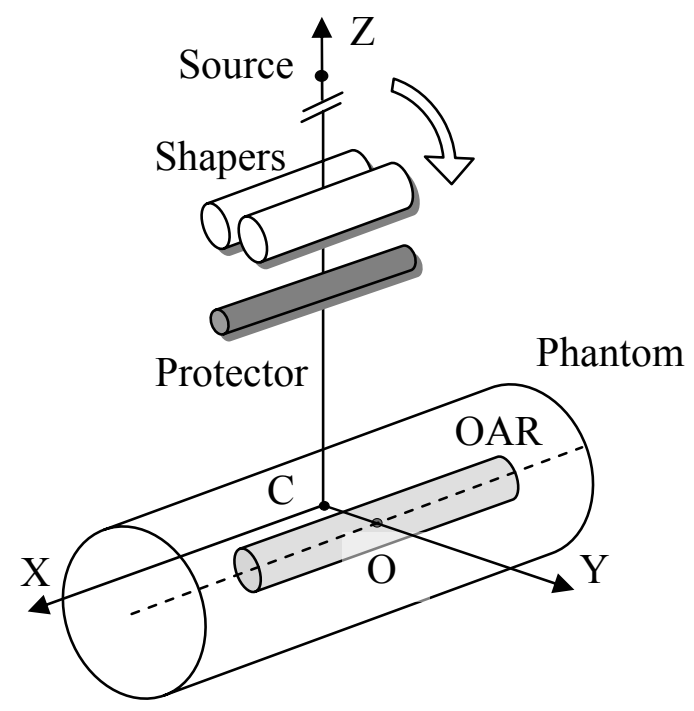

FIGURE 5-3. The neck phantom, containing the "spinal cord" as OAR, and the corresponding beam modifying devices, modeled by means of solid-geometry objects.

\subsubsection{Voxel-based neck model}

A realistic voxel-based neck model was created using CT images, taken from the U.S. National Library of Medicine's Visible Human Project ${ }^{\circledR}$ (2003), with their further transformation to a voxel-based geometry. The original CT data consisted of axial CT scans of the head taken at $1 \mathrm{~mm}$ intervals at a resolution of $512 \times 512$ pixels. Only the central part of the images with useful information $250 \times 250$ pixels in size was used. The obtained $3 \mathrm{D}$ matrix had dimensions of $250 \times 250 \times 100$ with a voxel size $1 \times 1 \times 1 \mathrm{~mm}^{3}$. Physical properties, such as tissue density and composition, were assigned to each voxel, according to the work of Schneider et al. (2000). Figure 5-4 shows the interrelation between the Hounsfield units and the mass density. Let us recall here, that Hounsfield unit $H$ was introduced in order to facilitate the intercomparison of $\mathrm{CT}$ values (linear attenuation coefficients) obtained from different $\mathrm{CT}$ scanners and with different X-ray beam energy spectra and is defined in such a way that water has always the value 0 and air the value -1000 :

$$
\mathrm{H}=\left(\frac{\mu}{\mu_{\mathrm{H}_{2} \mathrm{O}}}-1\right) \cdot 1000,
$$

where $\mu$ and $\mu_{\mathrm{H}_{2} \mathrm{O}}$ are the linear attenuation coefficients in an arbitrary medium and water. 


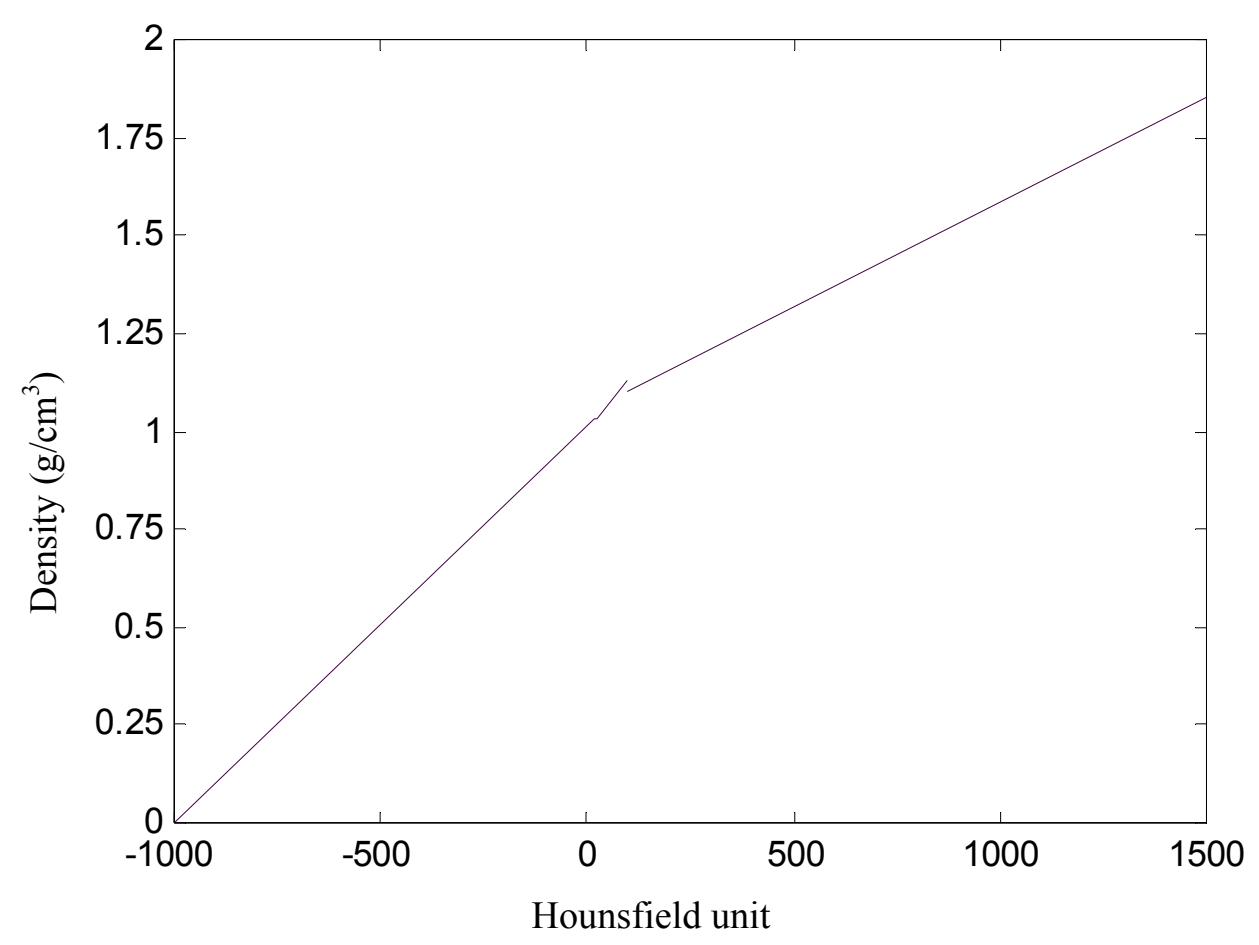

FIGURE 5-4. Conversion of the Hounsfield units to mass density

Misassignment of media can potentially lead to significant dose errors in Monte Carlo. Verhaegen and Devic (2005) observed dose errors in some regions of up to $10 \%$ for 6 and 15 MV photons due to inaccuracy of the extraction of material characteristics from CT images.

The choice of the PTV was based on the typical planning target volume for hypopharyngeal cancer, shown in Fig. 5-5 (a), which is concave in shape (Bratengeier et al. 2000). For simulations of the rotational irradiation the isocenter was chosen to be $32 \mathrm{~mm}$ anterior and $3 \mathrm{~mm}$ to the left relative to the center of the spinal cord (see Fig. 5-5(b)). The OAR was chosen to be $2.4 \mathrm{~cm}$ in diameter for more secure protection. The beam was collimated to a field size of $8 \times 8 \mathrm{~cm}^{2}$ at the isocenter. The origin of the coordinate system coincided with the isocenter and all the calculations of the beam modifying devices were done regarding to it. A complete gantry rotation with a discrete step of $10^{\circ}$ was applied.

\subsubsection{Simulations with solid-geometry neck model}

Simulation studies with the solid-geometry model had three purposes: (1) to verify the computations of the software tool, regarding the initial coordinates of the beam modifying devices and their motion dynamics, (2) to evaluate the calculated dose distributions, and (3) to 


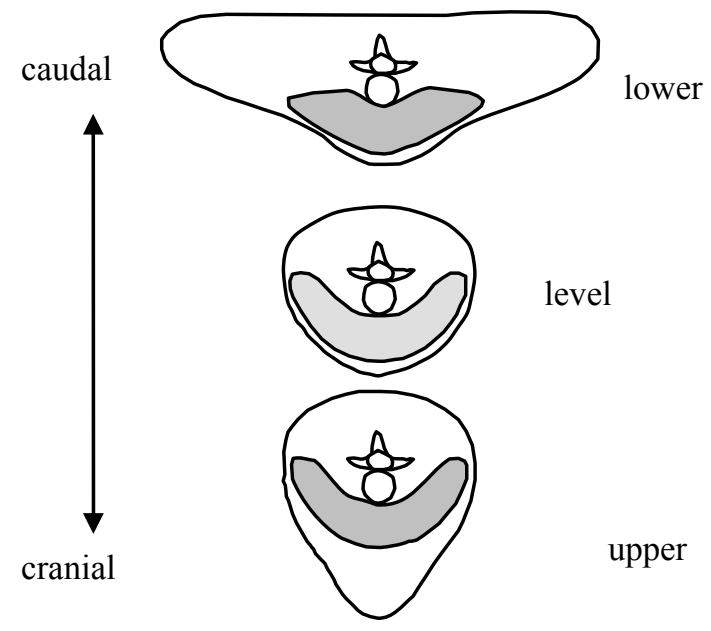

(a)

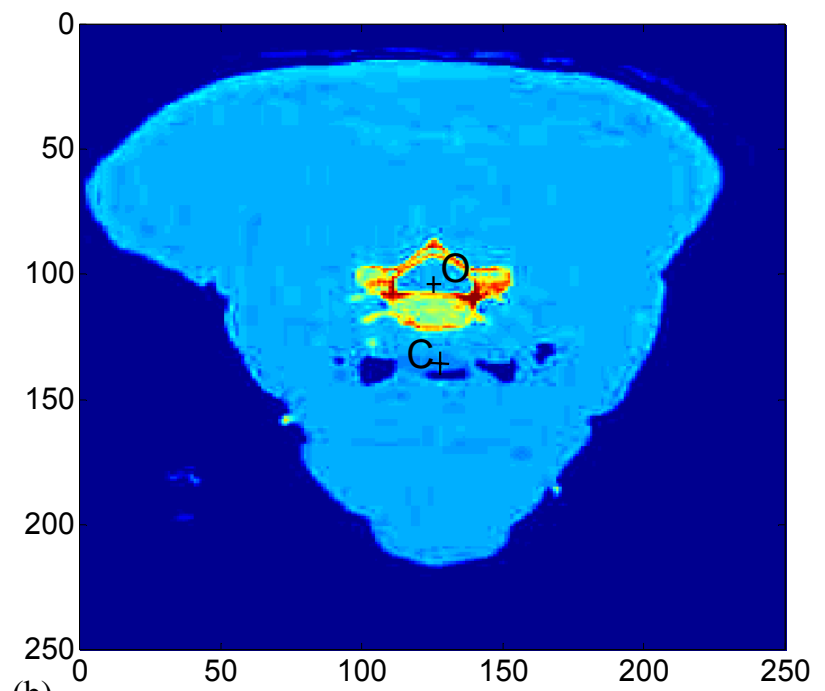

(b)

FIGURE 5-5. Schematic representation of the levels and shape of typical planning target volume of hypopharyngeal cancer (a) and the central slice of the voxel-based neck phantom (b). The arrangement of the isocenter (p. C) and the center of the OAR (p. O) are shown.

assess the two methods of the shapers' parameters calculations. The simulations related to the first two tasks refer to general evaluation of the efficiency of the beam modifying devices and the correct implementation of the motion geometry, while the simulations concerned the third task were performed for the evaluation of the dose distributions acquired by using shapers of the parameters calculated according to two different methods.

\subsubsection{Verification of the software module}

The verification of the calculations of the initial coordinates the beam modifying devices and their motion dynamics was performed through comparison of the results of the simulations with the published experimental data (Danciu 2001). An experimental irradiation setup and geometrical parameters of shapers and protectors were reproduced and final dose distributions were compared. A hardened $6 \mathrm{MV}$ beam was used in simulations, just as in the experimental measurements, in order to be able to compare the results. Simulated dose distributions were obtained using $2.6 \times 10^{9}$ photon histories, reducing the statistical uncertainty to less than $1 \%$. Like in the experimental setup, a lead protector and beam shapers from iroco wood (shown in Fig. 5-3) constituted the beam modulators. The geometrical parameters of the neck model and the calculated parameters of the corresponding beam modifying devices at $0^{\circ}$ gantry angle are listed in Table 5-1. 


\subsubsection{Studies of the dose distributions acquired using the beam modifying devices}

The number of simulations with solid-geometry phantom was done for studies of a performance of the beam modifying devices in terms of the achieved dose distributions and the possibilities offered by the beam modifying devices in rotational therapy.

(a) First study. In the first study, the simulations with the beam modulated only by a lead protector and by the same protector combined with two iroco wood beam shapers, with parameters listed in Table 5-1, were compared. The aim was to illustrate the degree of the dose nonuniformity outside the shielded region when the single protector is exploited and to estimate the improvement of the dose distribution in terms of uniformity when shapers are used. A $6 \mathrm{MV}$ hardened beam with $2.6 \times 10^{9}$ incident photons was used to simulate particle transport through the solid-geometry neck model.

TABLE 5-1. Parameters of the solid-geometry neck model and the beam modifying devices at $0^{\circ}$ gantry angle, corresponding to two irradiation setups: when the isocenter coincides with the center of the OAR $(\mathrm{CO}=0 \mathrm{~cm})$ and when the center of the OAR is shifted $2.5 \mathrm{~cm}$ along $\mathrm{y}$-axis relatively to the isocenter $(\mathrm{CO}=2.5 \mathrm{~cm})$. The origin point of the coordinate system coincides with the isocenter.

\begin{tabular}{|c|c|c|c|c|c|c|}
\hline \multirow[b]{2}{*}{$\begin{array}{l}\text { Object } \\
\text { name }\end{array}$} & \multicolumn{2}{|c|}{$\mathrm{CO}=0 \mathrm{~cm}$} & \multicolumn{2}{|c|}{$\mathrm{CO}=2.5 \mathrm{~cm}$} & \multirow{2}{*}{$\begin{array}{l}\text { Object } \\
\text { dimensions } \\
\mathrm{cm}\end{array}$} & \multirow[b]{2}{*}{$\begin{array}{l}\text { Object } \\
\text { material }\end{array}$} \\
\hline & $\begin{array}{l}\text { Object } \\
\text { center } \\
(\mathrm{x}, \mathrm{y}, \mathrm{z}), \mathrm{cm}\end{array}$ & $\begin{array}{l}\text { Shaft } \\
\text { coordinates } \\
(\mathrm{x}, \mathrm{y}, \mathrm{z}), \mathrm{cm}\end{array}$ & $\begin{array}{l}\text { Object } \\
\text { center } \\
(\mathrm{x}, \mathrm{y}, \mathrm{z}), \mathrm{cm}\end{array}$ & $\begin{array}{l}\text { Shaft } \\
\text { coordinates } \\
(\mathrm{x}, \mathrm{y}, \mathrm{z}), \mathrm{cm}\end{array}$ & & \\
\hline 1. Neck & $(0,0,0)$ & - & $(0,2.5,0)$ & 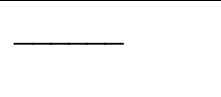 & $\begin{array}{l}\mathrm{R}=7.5^{*} \\
\mathrm{~L}=20.0\end{array}$ & Water \\
\hline $\begin{array}{l}\text { 2. Spinal } \\
\text { cord }\end{array}$ & $(0,0,0)$ & 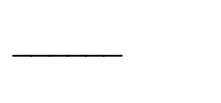 & $(0,2.5,0)$ & & $\begin{array}{l}\mathrm{R}=1.2 \\
\mathrm{~L}=10.0\end{array}$ & $\begin{array}{l}\text { Gray/white } \\
\text { matter }\end{array}$ \\
\hline $\begin{array}{l}\text { 3. Spinal } \\
\text { cord } \\
\text { protector }\end{array}$ & $(0,0,32.0)$ & $(0,0,32.0)$ & $(0,1.7,32.0)$ & $(0,0,32.0)$ & $\begin{array}{l}\mathrm{R}=0.8 \\
\mathrm{~L}=6.8\end{array}$ & Lead \\
\hline $\begin{array}{l}\text { 4. Left beam } \\
\text { shaper }\end{array}$ & $(0,-6.0,40.0)$ & $(0,-6.0,40.0)$ & $(0,-4.5,40.0)$ & $(0,-6.0,40.0)$ & $\begin{array}{l}\mathrm{R}=4.8 \\
\mathrm{~L}=6.0\end{array}$ & $\begin{array}{l}\text { Iroco } \\
\text { wood }\end{array}$ \\
\hline $\begin{array}{l}5 . \text { Right } \\
\text { beam shaper }\end{array}$ & $(0,6.0,40.0)$ & $(0,6.0,40.0)$ & $(0,7.5,40.0)$ & $(0,6.0,40.0)$ & $\begin{array}{l}\mathrm{R}=4.8 \\
\mathrm{~L}=6.0\end{array}$ & $\begin{array}{l}\text { Iroco } \\
\text { wood }\end{array}$ \\
\hline
\end{tabular}

*R- radius; L- length

(b) Second study. The second study was performed with the same assembly of the beam modifying devices with the parameters described in Table 5-1, but, additionally, except the hardened $6 \mathrm{MV}$ photon beam, using a $6 \mathrm{MV}$ beam with standard ELECTA SL75 spectrum and monoenergetic $2 \mathrm{MeV}$ beam, as a beam of a mean energy of $6 \mathrm{MV}$ polyenergetic beam 
(Mohan et al. 1985, Sheikh-Bagheri and Rogers 2002). The aim was to explore how the beam characteristics and the spectral model affect the dose distribution. The simulations were run using $2.6 \times 10^{9}, 4.4 \times 10^{9}$ and $4.4 \times 10^{8}$ photon histories for the corresponding irradiation beams.

\subsubsection{Assessment of the shapers' parameters calculation methods}

\subsection{1st method of shapers' parameters calculations: "From diameter to material”}

The method of the calculation of shapers' parameters is described in sect. 4.3.2.1. Initially, the central dose profile of the phantom, irradiated by the $6 \mathrm{MV}$ beam of hardened spectrum with the protector inserted in it, was explored. The desired dose build-up region was set to be $d_{b-u p}=4.2 \mathrm{~cm}$, that resulted in the unfiltered beam width $L N=2.5 \mathrm{~cm}$. The chosen distance between the shafts was selected to be $A_{1} A_{2}=12 \mathrm{~cm}$, resulting to the shaper diameter equal to $12-2.5=9.5 \mathrm{~cm}$ (i.e., $\mathrm{d}=\mathrm{A}_{1} \mathrm{~A}_{2}-\mathrm{LN}$ ). When the isocenter coincides with the center of the OAR (i.e., $\mathrm{CO}=0$ ), the average filtration chord for the complete gantry rotation of $360^{\circ}$ every $2^{\circ}$, calculated as described in appendix $B$, for the point of maximal dose $\left(\mathrm{y}_{\mathrm{p}}=4.7 \mathrm{~cm}\right)$ was $\mathrm{h}=4.0 \mathrm{~cm}$. The desired dose reduction was chosen to be from $94 \%$ to $82 \%$ (see Fig. 5 6(a)). The calculated linear attenuation coefficient of the material that gives the desired dose reduction was $0.0346 \mathrm{~cm}^{-1}$. For $\mathrm{CO}=2.5 \mathrm{~cm}$, the average filtration chord of the beam that passes through the point of maximal dose $\left(y_{p}=-3.9 \mathrm{~cm}\right.$, that is $3.9 \mathrm{~cm}$ from the isocenter or $6.4 \mathrm{~cm}$ from the center of the OAR) was $5.4 \mathrm{~cm}$ and the desired dose reduction was from $99 \%$ to $82 \%$ (see Fig. 5-6(b)). These parameters led to the linear attenuation coefficient $0.035 \mathrm{~cm}^{-1}$. The material with the closest weighted mean linear attenuation coefficient to the both calculated values was found to be an iroco wood, as it can be seen from Table 5-2, which has $\bar{\mu}=0.031 \mathrm{~cm}^{-1}$ for the hardened $6 \mathrm{MV}$ spectrum.

According to the work of Danciu (2001), an alternative material of the shapers was also used. To achieve the same filtration offered by iroco wood of density $\rho=0.71 \mathrm{~g} / \mathrm{cm}^{3}$, the new material should offer about the same surface density to the beam transmitted along the disk 

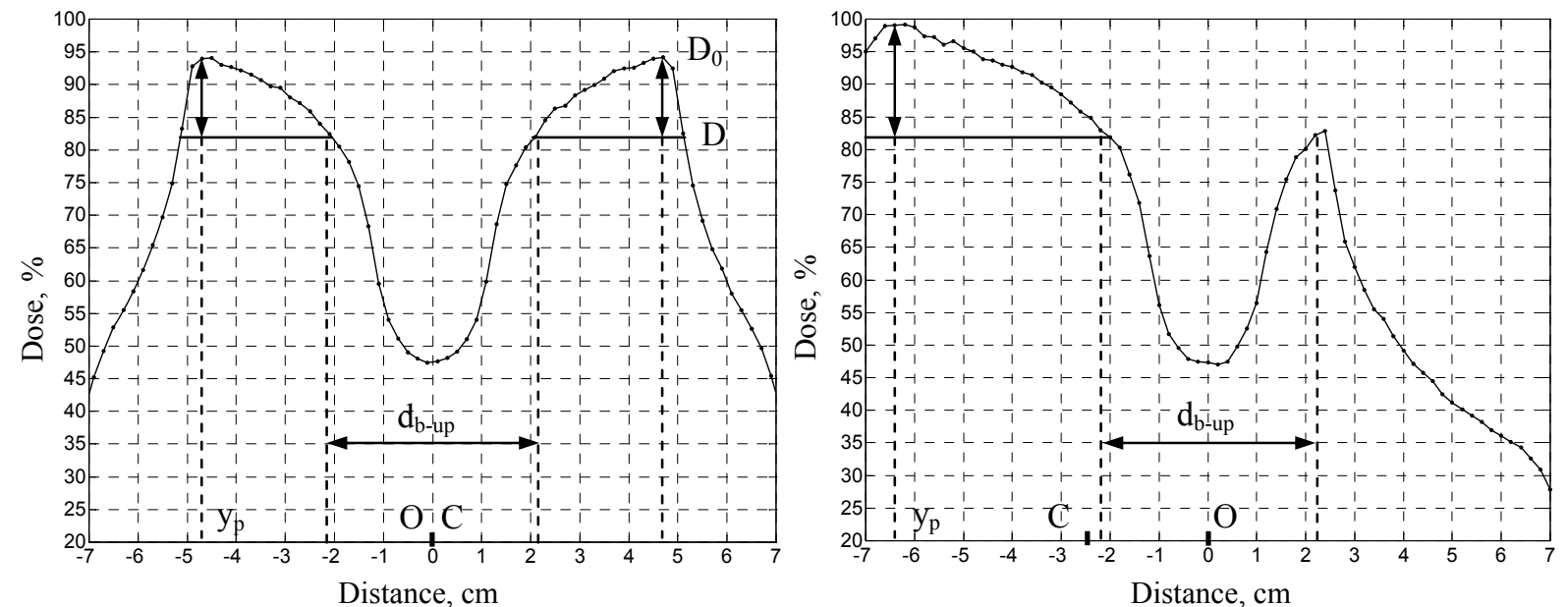

FIGURE 5-6. Central dose profiles for the beams modulated with the single lead protector for cases in which the isocenter coincides with the center of the OAR (a) and when they are shifted $2.5 \mathrm{~cm}$ relative to each other (b). The initial dose value and the desired one are pointed out as $\mathrm{D}_{0}$ and $\mathrm{D}$, respectively, $\mathrm{C}$ is the isocenter and $\mathrm{O}$ is the center of the $\mathrm{OAR}, \mathrm{y}_{\mathrm{p}}$ is the $\mathrm{y}$-coordinate of the maximal dose point.

diameter. Thus, $\mathrm{d}_{\text {polyst }}=\left(\mathrm{d}_{\text {iroco }} \rho_{\text {iroco }}\right) / \rho_{\text {polyst }}$ or $\mathrm{d}_{\text {polyst }}=(9.5 \cdot 0.71) / 1.04=6.5 \mathrm{~cm}$. Indeed, the linear attenuation coefficient of the shapers of $6.5 \mathrm{~cm}$ in diameter and the distance between the shapers edges $\mathrm{LN}=2.5 \mathrm{~cm}$, and for dose reduction from $94 \%$ to $82 \%$ at $\mathrm{y}_{\mathrm{p}}=4.7 \mathrm{~cm}$ from the center of the OAR (which leads to an average filtration chord length $3.2 \mathrm{~cm}$ ) is $\bar{\mu}=0.0432$ $\mathrm{cm}^{-1}$ (see Table 5-2). This value is close to the weighted mean linear attenuation coefficients of polystyrene, polyethylene and paraffin for the hardened $6 \mathrm{MV}$ spectrum. In the case of $\mathrm{CO}=2.5 \mathrm{~cm}$, the desired dose reduction from $99 \%$ to $82 \%$ at $64 \mathrm{~mm}$ from the center of the OAR results in an average beam filtration of $4.2 \mathrm{~cm}$. The linear attenuation coefficient, calculated as $\left[-\ln \left(\mathrm{D} / \mathrm{D}_{0}\right) / \overline{\mathrm{h}}\right]$, that agrees to these conditions is $\bar{\mu}=0.0451 \mathrm{~cm}^{-1}$, which also indicates that closest linear attenuation coefficient to the calculated one is that of polystyrene.

Simulation studies with two configurations of beam modifying devices, containing shapers of different materials, were carried out to verify the above described approach in term of dose distributions. The irradiation setups with the isocenter coinciding with the center of the OAR and shifted $2.5 \mathrm{~cm}$ regarding to it, were used in these simulations. In both cases the protector was the same lead cylinder, while the beam shapers were of iroco wood and polystyrene with geometrical parameters and initial coordinates, described above and summarized in Table 5-3. Dose distributions were obtained with $2.6 \times 10^{9}$ photon histories. 
TABLE 5-2. The weighted mean linear attenuation coefficients (for hardened $6 \mathrm{MV}$ spectrum) for a number of low density materials.

\begin{tabular}{|c|c|c|c|}
\hline Material & Density, g/cm3 & Composition & $\mu, \mathrm{cm}^{-1}$ \\
\hline $\begin{array}{l}\text { A-150 Tissue- } \\
\text { Equivalent } \\
\text { Plastic }^{1}\end{array}$ & 1.127 & $\begin{array}{l}\text { H: } 0.101330 \\
\text { C: } 0.775498 \\
\text { N: } 0.035057 \\
\text { O: } 0.052315 \\
\text { F: } 0.017423 \\
\text { Ca: } 0.018377\end{array}$ & 0.0503 \\
\hline $\begin{array}{l}\text { Polymethyl } \\
\text { Methacrylate } \\
\text { (PMMA) }^{1}\end{array}$ & 1.19 & $\begin{array}{l}\text { H: } 0.080541 \\
\text { C: } 0.599846 \\
\text { O: } 0.319613\end{array}$ & 0.0518 \\
\hline Polyethylene $^{1}$ & 0.93 & $\begin{array}{l}\text { H: } 0.143716 \\
\text { C: } 0.856284\end{array}$ & 0.0427 \\
\hline Iroco wood $^{2}$ & 0.71 & $\begin{array}{l}\text { H: } 0.8045 \\
\text { C: } 0.0838 \\
\text { O: } 0.1117\end{array}$ & 0.0310 \\
\hline Polystyrene $^{1}$ & 1.06 & $\begin{array}{l}\text { H: } 0.077421 \\
\text { C: } 0.922579\end{array}$ & 0.0454 \\
\hline Paraffin wax ${ }^{3}$ & 0.93 & $\begin{array}{l}\text { H: } 0.148605 \\
\text { C: } 0.851395\end{array}$ & 0.0428 \\
\hline $\begin{array}{l}\text { Polyvinyl } \\
\text { chloride (PVC) }\end{array}$ & 1.3 & $\begin{array}{l}\text { H: } 0.048380 \\
\text { C: } 0.384360 \\
\text { Cl: } 0.567260\end{array}$ & 0.0551 \\
\hline
\end{tabular}

\subsection{2nd method of shapers' parameters calculations: "From material to diameter"}

For a verification of this shapers' parameters calculations method, described in sect. 4.3.2.2, four simulations studies were carried out. The intent of the simulations performed was to assess the accuracy of the achieved dose distributions. Here, the term of accuracy will imply how well the acquired dose distribution corresponds to the desired one. The simulations were performed for a rotational mode with the full assembly of beam modifying devices. The protector used was the same as in previous studies with the parameters and initial coordinates, presented in Table 5-3. The features extracted from the central dose profile of a rotational beam modulated with single cylindrical protector as well as calculated parameters and initial coordinates of the shapers exploited in the simulations of this section are presented in Table 54. The method was tested for polyenergetic and monoenergetic beams, different distances between the isocenter and the center of the OAR, different dose reductions and dose build-ups 
TABLE 5-3. Parameters of the beam modifying devices at $0^{\circ}$ gantry angle for two irradiation setups: (i) the isocenter coincides with the center of the OAR $(\mathrm{CO}=0 \mathrm{~cm})$ and (ii) they are shifted $2.5 \mathrm{~cm}$ relative to each other $(\mathrm{CO}=2.5 \mathrm{~cm})$. The shapers were modeled using cylindrical solidgeometry objects.

\begin{tabular}{|c|c|c|c|c|c|}
\hline & $\begin{array}{l}\text { Object } \\
\text { name }\end{array}$ & $\begin{array}{l}\text { Object center } \\
(\mathrm{x}, \mathrm{y}, \mathrm{z}) \\
\mathrm{cm}\end{array}$ & $\begin{array}{l}\text { Coordinates } \\
\text { of shaft } \\
(\mathrm{x}, \mathrm{y}, \mathrm{z}), \mathrm{cm}\end{array}$ & $\begin{array}{l}\text { Object } \\
\text { dimensions } \\
(\mathrm{x}, \mathrm{y}, \mathrm{z}), \mathrm{cm}\end{array}$ & $\begin{array}{l}\text { Object } \\
\text { material }\end{array}$ \\
\hline \multirow{5}{*}{$\begin{array}{l}\tilde{E} \\
0 \\
0 \\
0 \\
0\end{array}$} & 1. Protector & $(0,0,32.0)$ & $(0,0,32.0)$ & $\mathrm{R}=0.8, \mathrm{~L}=6.8^{*}$ & Lead \\
\hline & 2a. Left beam shaper & $(0,-6.0,40.0)$ & $(0,-6.0,40.0)$ & $\mathrm{R}=4.8, \mathrm{~L}=6.0$ & Iroco wood \\
\hline & 2b. Left beam shaper & $(0,-4.5,40.0)$ & $(0,-4.5,40.0)$ & $\mathrm{R}=3.3, \mathrm{~L}=6.0$ & Polystyrene \\
\hline & 3a. Right beam shaper & $(0,6.0,40.0)$ & $(0,6.0,40.0)$ & $\mathrm{R}=4.8, \mathrm{~L}=6.0$ & Iroco wood \\
\hline & 3b. Right beam shaper & $(0,4.5,40.0)$ & $(0,4.5,40.0)$ & $\mathrm{R}=3.3, \mathrm{~L}=6.0$ & Polystyrene \\
\hline \multirow{5}{*}{$\begin{array}{l}\text { E } \\
n \\
i \\
11 \\
0\end{array}$} & 1. Protector & $(0,1.7,32.0)$ & $(0,0,32.0)$ & $\mathrm{R}=0.8, \mathrm{~L}=6.8$ & Lead \\
\hline & 2a. Left beam shaper & $(0,-4.5,40.0)$ & $(0,-6.0,40.0)$ & $\mathrm{R}=4.8, \mathrm{~L}=6.0$ & Iroco wood \\
\hline & 2b. Left beam shaper & $(0,-3.0,40.0)$ & $(0,-4.5,40.0)$ & $\mathrm{R}=3.3, \mathrm{~L}=6.0$ & Polystyrene \\
\hline & 3a. Right beam shaper & $(0,7.5,40.0)$ & $(0,6.0,40.0)$ & $\mathrm{R}=4.8, \mathrm{~L}=6.0$ & Iroco wood \\
\hline & 3b. Right beam shaper & $(0,6.0,40.0)$ & $(0,4.5,40.0)$ & $\mathrm{R}=3.3, \mathrm{~L}=6.0$ & Polystyrene \\
\hline
\end{tabular}

${ }^{*} \mathrm{R}$ - radius, L- length

as well as two shapers' materials. The number of photon histories was $8.7 \times 10^{8}$ for $2 \mathrm{MeV}$, except 2 nd experiment, which was performed using $3.5 \times 10^{9}$ photons, and $4.4 \times 10^{8}$ for 6 MV beam.

\subsubsection{Simulations with voxel-based neck model}

In the application study with the voxel-based neck model, described in sect. 5.2.4.2, and the whole assembly of beam modifying devices, the phantom was irradiated with a $6 \mathrm{MV}$ hardened beam, using in total $6.5 \times 10^{8}$ photon histories. The beam modifying devices were modeled by means of solid-geometry objects. Their parameters and initial coordinates at $0^{\circ}$ gantry angle are summarized in Table 5-5.

In all simulations, except if it was designated otherwise, photon fan beams, originated from a point source of size $1 \times 1 \mathrm{~mm}^{2}, 100 \mathrm{~cm}$ away from the isocenter, were used. For the MC simulations, the electron and photon cutoff energies were set to 0.1 and $0.01 \mathrm{MeV}$, respectively. Production of bremsstrahlung photons and knock-on electrons were considered for energies above $0.01 \mathrm{MeV}$. The fractional energy loss step was chosen to be $4 \%$ for the 
TABLE 5-4. Features of the desired dose distribution and resultant parameters of shapers and their initial coordinates.

\begin{tabular}{lllll}
\hline \hline Input data: & 1 st experiment & 2nd experiment & 3rd experiment & 4th experiment \\
\hline $\mathrm{CO}, \mathrm{cm}$ & 2.5 & 0 & 2.5 & 0 \\
Energy, MV & $2 \mathrm{MeV}$ & $2 \mathrm{MeV}$ & $6 \mathrm{MV}$ (hard) & $2 \mathrm{MeV}$ \\
$\mu, \mathrm{cm}^{-1}$ & 0.0339 & 0.0339 & 0.0308 & 0.0497 \\
$\mathrm{~d}_{\mathrm{b}-\mathrm{up}, \mathrm{cm}}$ & 4.2 & 3.6 & 4.2 & 4.2 \\
$\mathrm{D} / \mathrm{D}_{0}$ & $82 / 99$ & $80 / 95$ & $82 / 99$ & $82 / 95$ \\
$\mathrm{y}_{\mathrm{p}}, \mathrm{cm}$ & -39 & 45 & -39 & 45 \\
Material & iroco & iroco & iroco & polystyrene \\
Calculated data: & & & & \\
\hline $\mathrm{d}, \mathrm{cm}$ & 10.0 & 13.3 & 11.7 & 6.4 \\
$\mathrm{~L}, \mathrm{~cm}$ & 6.0 & 6.0 & 6.0 & 6.0 \\
$\mathrm{~A}_{1} \mathrm{~A}_{2}$, cm & 12.5 & 15.5 & 14.2 & 8.9 \\
Aver. chord, cm & 5.6 & 5.1 & 6.1 & 3.0 \\
Initial shapers' & $(0,-6.3 ; 40)$ & $(0,-7.7 ; 40)$ & $(0,-7.1 ; 40)$ & $(0,-4.4 ; 40)$ \\
axes coord., cm & $(0,6.3 ; 40)$ & $(0,7.7 ; 40)$ & $(0,7.1 ; 40)$ & $(0,4.4 ; 40)$ \\
Initial shapers' & $(0,-4.8 ; 40)$ & $(0,-7.7 ; 40)$ & $(0,-5.6 ; 40)$ & $(0,-4.4 ; 40)$ \\
centers coord., cm & $(0,7.8 ; 40)$ & $(0,7.7 ; 40)$ & $(0,8.6 ; 40)$ & $(0,4.4 ; 40)$ \\
\hline \hline
\end{tabular}

Abbreviations used in the table: $C O$ is the distance between the isocenter and the center of the OAR, $\mu$ is (weighted mean) linear attenuation coefficient, $d_{b-u p}$ is dose build-up region, $y_{p}$ is maximal dose point coordinate, $d$ and $L$ are shaper's diameter and length, respectively, and $A_{1} A_{2}$ is distance between the shafts of shapers.

simulations with the solid-geometry model and $10 \%$ for the simulations with the voxel-based model. Dose distributions were obtained using different number of photon histories in order to obtain $1 \%$ statistical uncertainty of the simulated results. The dose matrices were defined with a voxel resolution of $2 \times 2 \times 2 \mathrm{~mm}^{3}$.

TABLE 5-5. Parameters of the beam modifying devices at $0^{\circ}$ gantry angle for simulations with the voxel-based neck model. The beam modifying devices were modeled using cylindrical solidgeometry objects. The origin point of the coordinates coincides with the isocenter.

\begin{tabular}{lllll}
\hline \hline $\begin{array}{l}\text { Object } \\
\text { name }\end{array}$ & $\begin{array}{l}\text { Object center } \\
(\mathrm{x}, \mathrm{y}, \mathrm{z}), \mathrm{cm}\end{array}$ & $\begin{array}{l}\text { Coordinates of } \\
\text { shaft }(\mathrm{x}, \mathrm{y}, \mathrm{z}), \mathrm{cm}\end{array}$ & $\begin{array}{l}\text { Object } \\
\text { dimensions, cm }\end{array}$ & $\begin{array}{l}\text { Object } \\
\text { material }\end{array}$ \\
\hline $\begin{array}{l}\text { Spinal cord } \\
\text { protector }\end{array}$ & $(0,-2.0,34.2)$ & $(0,0,32.0)$ & $\begin{array}{l}\mathrm{R}=0.8^{*} \\
\mathrm{~L}=6.8\end{array}$ & Lead \\
$\begin{array}{l}\text { Left beam } \\
\text { shaper }\end{array}$ & $(0,-6.2,41.9)$ & $(0,-6.0,40.0)$ & $\begin{array}{l}\mathrm{R}=4.8 \\
\mathrm{~L}=6.0\end{array}$ & Iroco wood \\
$\begin{array}{l}\text { Right beam } \\
\text { shaper }\end{array}$ & $(0,5.8,41.9)$ & $(0,6.0,40.0)$ & $\begin{array}{l}\mathrm{R}=4.8 \\
\mathrm{~L}=6.0\end{array}$ & Iroco wood \\
\hline \hline
\end{tabular}

"R- radius, L- length 
The dose computational times per $10^{7}$ photon histories run on a Intel Pentium IV PC with two dual-core processors with 4Gb RAM, operating at 2,4 GHz under Windows XP are generalized in the Table 5-6.

TABLE 5-6. Computational times of 3D dose matrices for different beam energies and phantoms involved. The time is given per $10^{7}$ photon histories.

\begin{tabular}{|c|c|c|c|}
\hline $\begin{array}{l}\text { Beam } \\
\text { energy/spectrum }\end{array}$ & Phantom involved & Beam modifying devices & $\begin{array}{l}\text { Computational } \\
\text { time, } \min / 10^{7} \\
\text { photons }\end{array}$ \\
\hline \multirow{3}{*}{$\begin{array}{l}2 \mathrm{MeV} \\
\text { monoenergetic }\end{array}$} & \multirow{3}{*}{$\begin{array}{l}\text { Solid-geometry water } \\
\text { phantom } \\
(\mathrm{R}=7.5 \mathrm{~cm}, \mathrm{~L}=20 \mathrm{~cm})\end{array}$} & Open beam $\left(10 \times 10 \mathrm{~cm}^{2}\right)$ & $1 \mathrm{~h} 22 \mathrm{~min}$ \\
\hline & & Lead protector $(\mathrm{R}=0.8 \mathrm{~cm}, \mathrm{~L}=10 \mathrm{~cm})$ & $1 \mathrm{~h} 43 \mathrm{~min}$ \\
\hline & & $\begin{array}{l}\text { Lead protector }(\mathrm{R}=0.8 \mathrm{~cm}, \mathrm{~L}=6.8 \mathrm{~cm}) \\
+ \text { iroco shapers }(\mathrm{R}=4.6 \mathrm{~cm}, \mathrm{~L}=6.0 \mathrm{~cm})\end{array}$ & $2 \mathrm{~h} 50 \mathrm{~min}$ \\
\hline \multirow{4}{*}{$\begin{array}{l}6 \mathrm{MV}, \text { hardened } \\
\text { spectrum }\end{array}$} & \multirow{3}{*}{$\begin{array}{l}\text { Solid-geometry water } \\
\text { phantom } \\
(\mathrm{R}=7.5 \mathrm{~cm}, \mathrm{~L}=20 \mathrm{~cm})\end{array}$} & Open beam $\left(10 \times 10 \mathrm{~cm}^{2}\right)$ & $47 \mathrm{~min}$ \\
\hline & & Lead protector $(\mathrm{R}=0.8 \mathrm{~cm}, \mathrm{~L}=10 \mathrm{~cm})$ & $50 \mathrm{~min}$ \\
\hline & & $\begin{array}{l}\text { Lead protector }(\mathrm{R}=0.8 \mathrm{~cm}, \mathrm{~L}=6.8 \mathrm{~cm}) \\
+ \text { iroco shapers }(\mathrm{R}=4.6 \mathrm{~cm}, \mathrm{~L}=6.0 \mathrm{~cm})\end{array}$ & 1h $21 \mathrm{~min}$ \\
\hline & Voxel-based phantom & $\begin{array}{l}\text { Lead protector }(\mathrm{R}=0.8 \mathrm{~cm}, \mathrm{~L}=6.8 \mathrm{~cm}) \\
+ \text { iroco shapers }(\mathrm{R}=4.6 \mathrm{~cm}, \mathrm{~L}=6.0 \mathrm{~cm})\end{array}$ & $10 \mathrm{~h} 16 \mathrm{~min}$ \\
\hline
\end{tabular}

The time values presented in the table are given to illustration purposes and are dependent on the load of the computer in some degree. The exact time evaluation and comparison was beyond the scope of the work

\subsection{RESULTS:}

\subsubsection{Verification of the software module}

Figure 5-7(a) presents a comparison of simulated and published experimental dose profiles in the case wherein the isocenter is coinciding with the center of the OAR. Central dose profiles were used for comparison. Curve A represents the open beam dose profile, while curve $\mathrm{B}$ represents the dose profile of a beam modulated with the beam modifying devices. The dose values in this study and all following studies were normalized to the dose at the isocenter for the open beam (profile A). Similarly, Figure 5-7(b) shows the corresponding comparison for the irradiation setup, where the isocenter was shifted $2.5 \mathrm{~cm}$ with regard to the center of the OAR. The results of both irradiation setup arrangements show discrepancies of less than $3 \%$ between simulated and experimental data. 

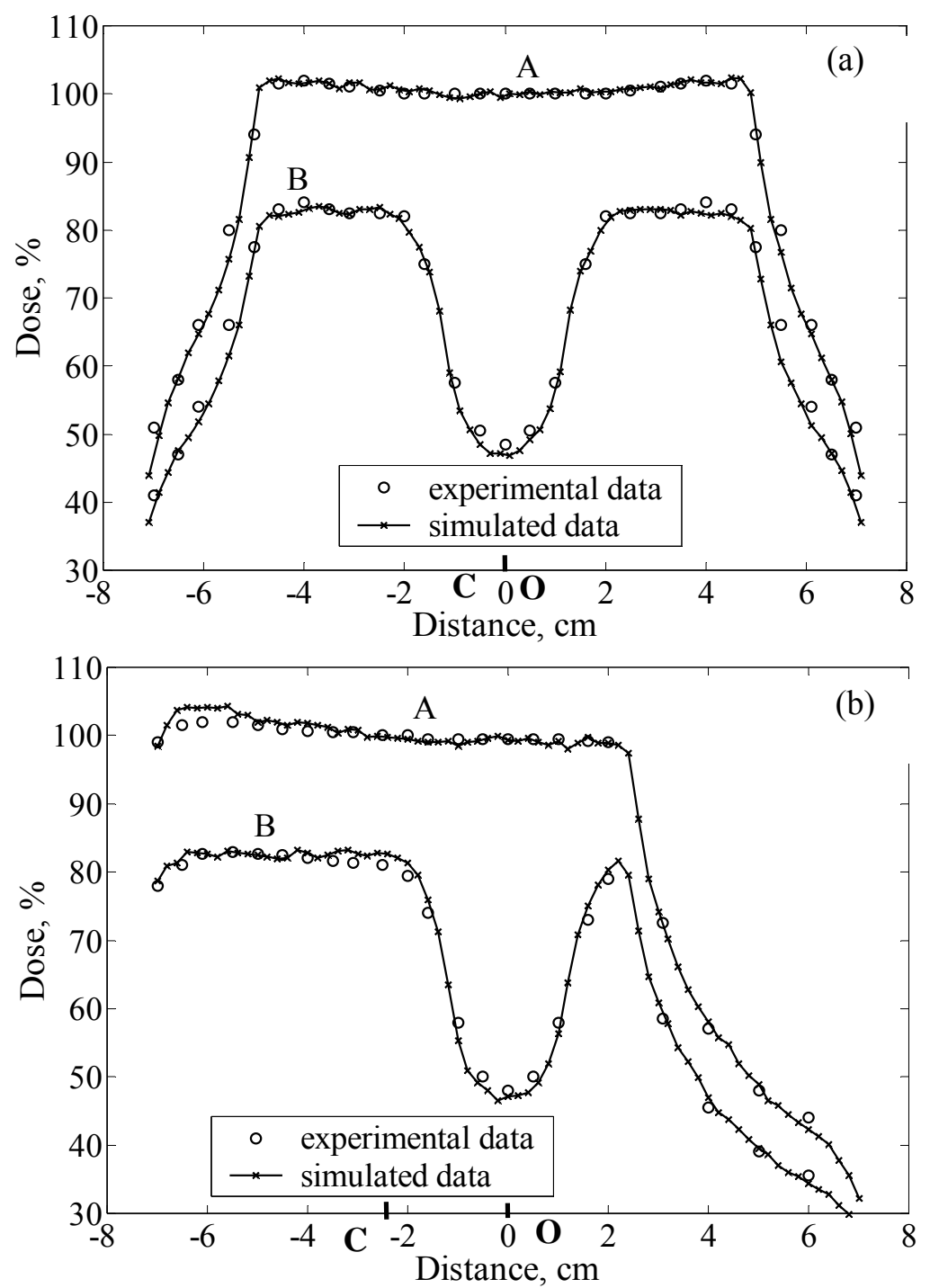

FIGURE 5-7. Comparison of simulated and experimental data for rotational therapy with a hardened $6 \mathrm{MV}$ beam for the irradiation setup, when the isocenter coincides with the center of the OAR (a) and when they are shifted $2.5 \mathrm{~cm}$ relative to each other (b). The modulation of the beam is accomplished with the cylindrical lead protector and iroco wood shapers. The curve A represents the transverse dose profile at the center of the cylindrical neck model of the open beam; curve B represents the dose profile of the beam modulated with the beam modifying devices.

\subsubsection{Studies of the dose distributions acquired using the beam modifying devices}

The importance of the beam shapers is illustrated by Fig. 5-8. Figure 5-8(a) shows transverse dose profiles for the irradiation setup with the isocenter coinciding with the center of the OAR, obtained from the simulations with: (i) an open beam (profile A), (ii) a beam shaped by the lead protector (profile C), and (iii) a beam shaped by the full assembly of the beam modifying devices (profile B). Similarly, Fig. 5-8(b) depicts simulated dose profiles when the isocenter is shifted regarding to the center of the OAR. 

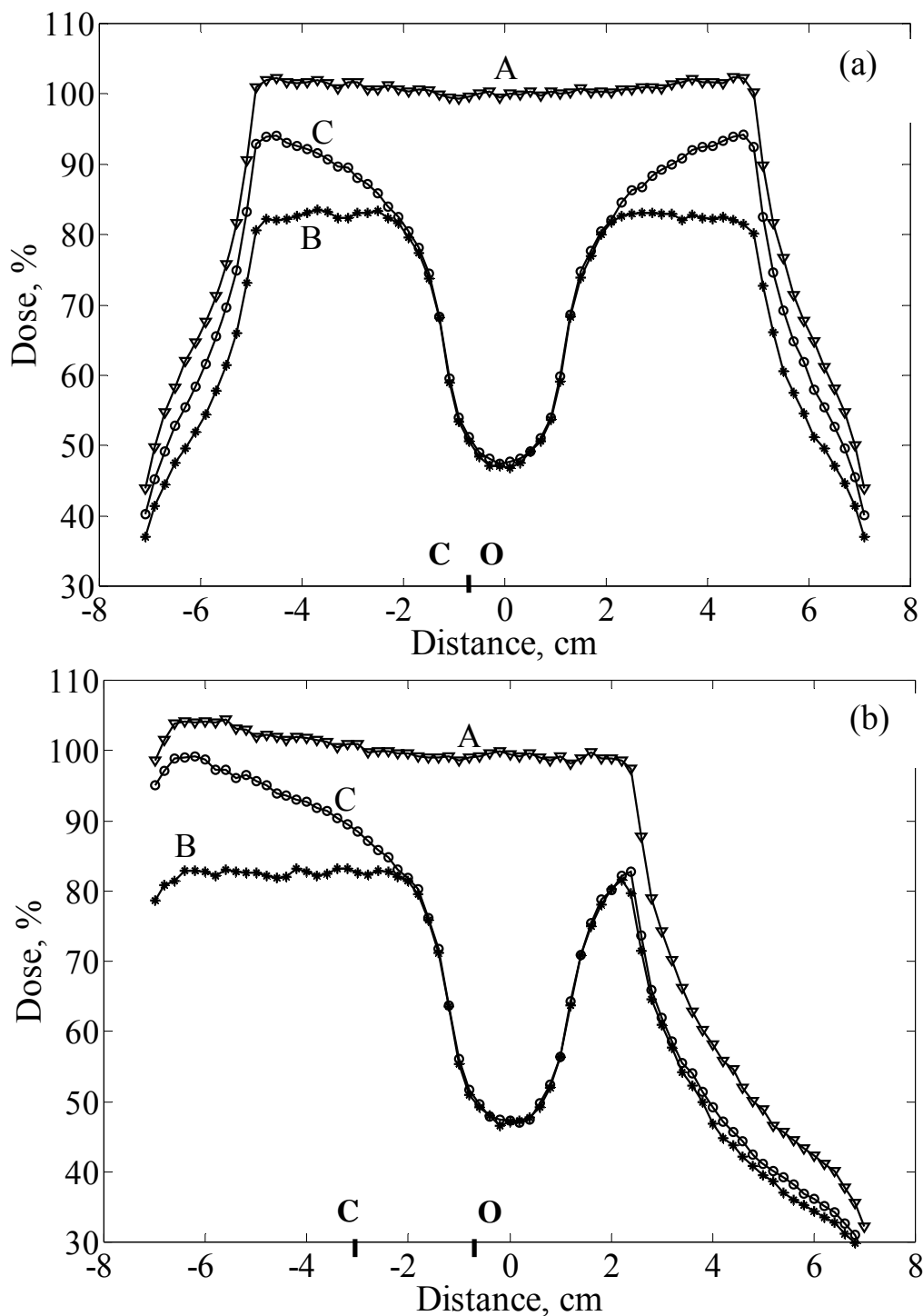

FIGURE 5-8. Beam shaping with beam modifying devices in rotational therapy for the cases in which the isocenter coincides with the center of the OAR (a) and when they are shifted $2.5 \mathrm{~cm}$ relative to each other (b). The curve A represents transverse dose profile at the center of the cylindrical neck model for the open beam, curve B the beam modulated with the lead protector and iroco wood shapers, and curve $\mathrm{C}$ represents the dose profile for the beam modulated with the single lead protector. A hardened beam of $6 \mathrm{MV}$ was used for simulations.

The isodose profiles of the central phantom's cross section of the same simulations are presented in Figs. 5-9(a) to 5-9(d). Figures 5-9(a) and 5-9(b) show the dose distributions received with the single protector for $\mathrm{CO}=0 \mathrm{~cm}$ and $\mathrm{CO}=2.5 \mathrm{~cm}$, respectively. While Figs. 58(c) and 5-8(d) depict dose distributions simulated using the complete assembly of beam modifying devices for the same isocenter arrangements.

The results show identical protection of the OAR using either a single protector or a full assembly of beam modifying devices. The protection provided is in the range of $47 \%$ at the center of the OAR to $64 \%$ at the periphery of the OAR (or $57 \%$ to $77.5 \%$, if the dose is 

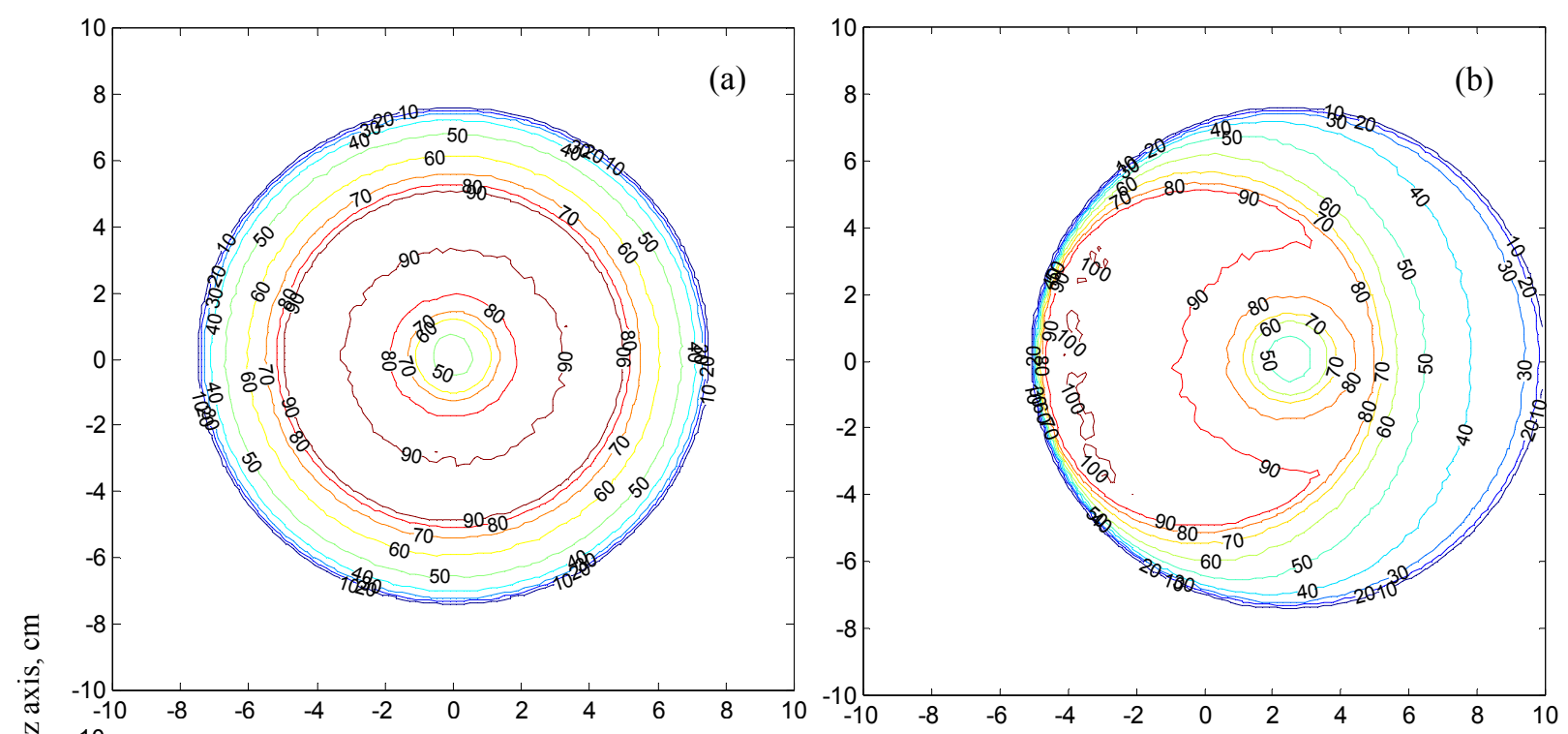

过
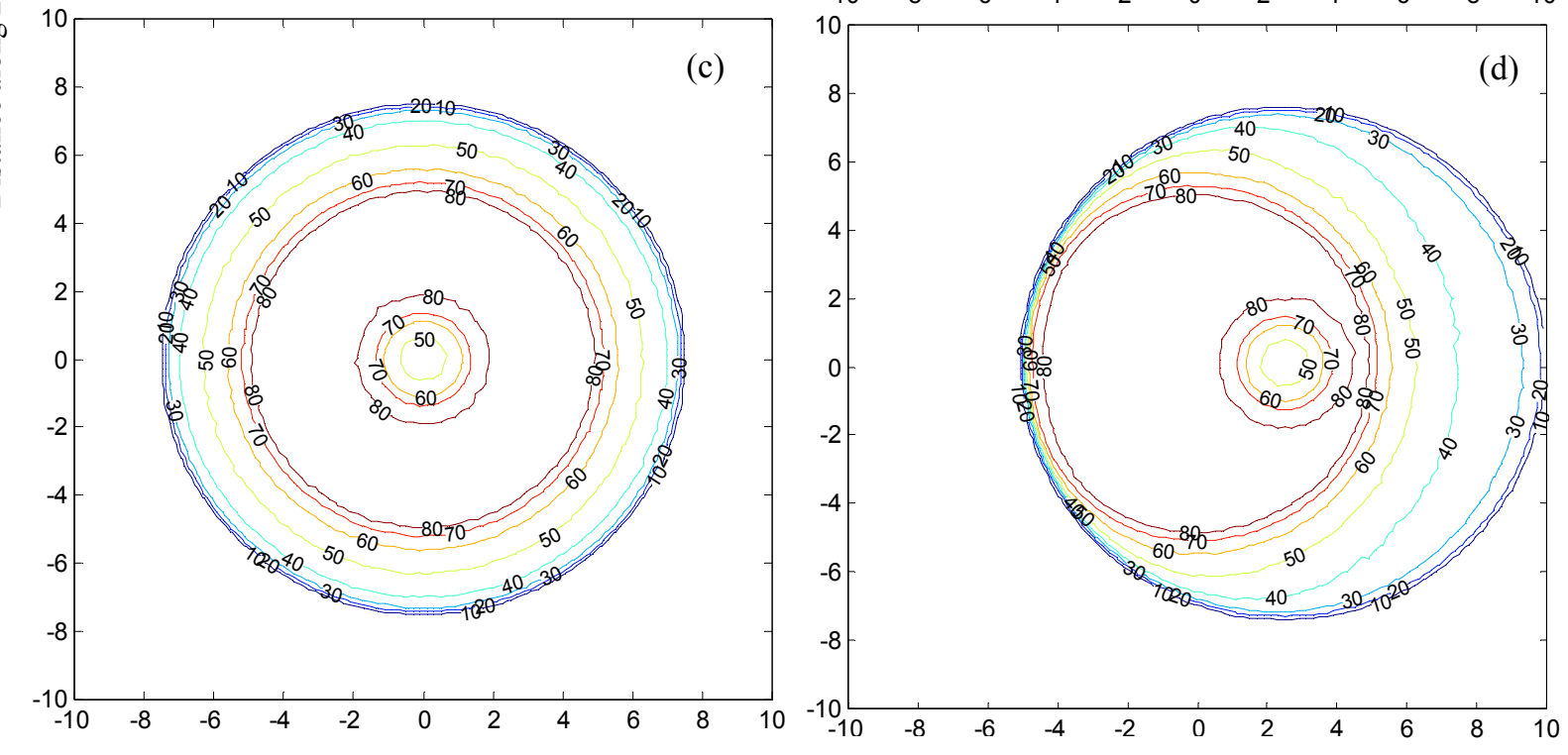

Distance along y axis, $\mathrm{cm}$

FIGURE 5-9. The isodose profiles at the central cross section of the solid-geometry neck phantom for rotational irradiation. In the upper row the isodose profiles of the beam modulated with the single lead protector inserted in it for the case in which the isocenter coincides with the center of the OAR (a) and for the case when they are shifted $2.5 \mathrm{~cm}$ relatively to each other (b) are shown. The isodose profiles, presented in the lower row are of the beam modulated with the lead protector and iroco beam shapers for $\mathrm{CO}=0 \mathrm{~cm}$ (c) and for $\mathrm{CO}=2.5 \mathrm{~cm}(\mathrm{~d})$. A hardened beam of $6 \mathrm{MV}$ was used for simulations.

normalized to the average dose in the PTV). Improved dose distribution in the PTV in terms of uniformity (curve B versus curve $C$ ) is obtained by adding the beam shapers to the protector. As is shown in Fig. 5-8 (curves B) a region of almost uniform dose distribution (with inhomogeneity of $0.6 \%$ ) starts $9 \mathrm{~mm}$ beyond the OAR.

In the second study of the system performance, the comparison of the achieved dose distributions by means of the whole assembly of the beam modifying devices using the beams of different energies and spectral characteristics was done. The results are shown in Fig. 5-10. 

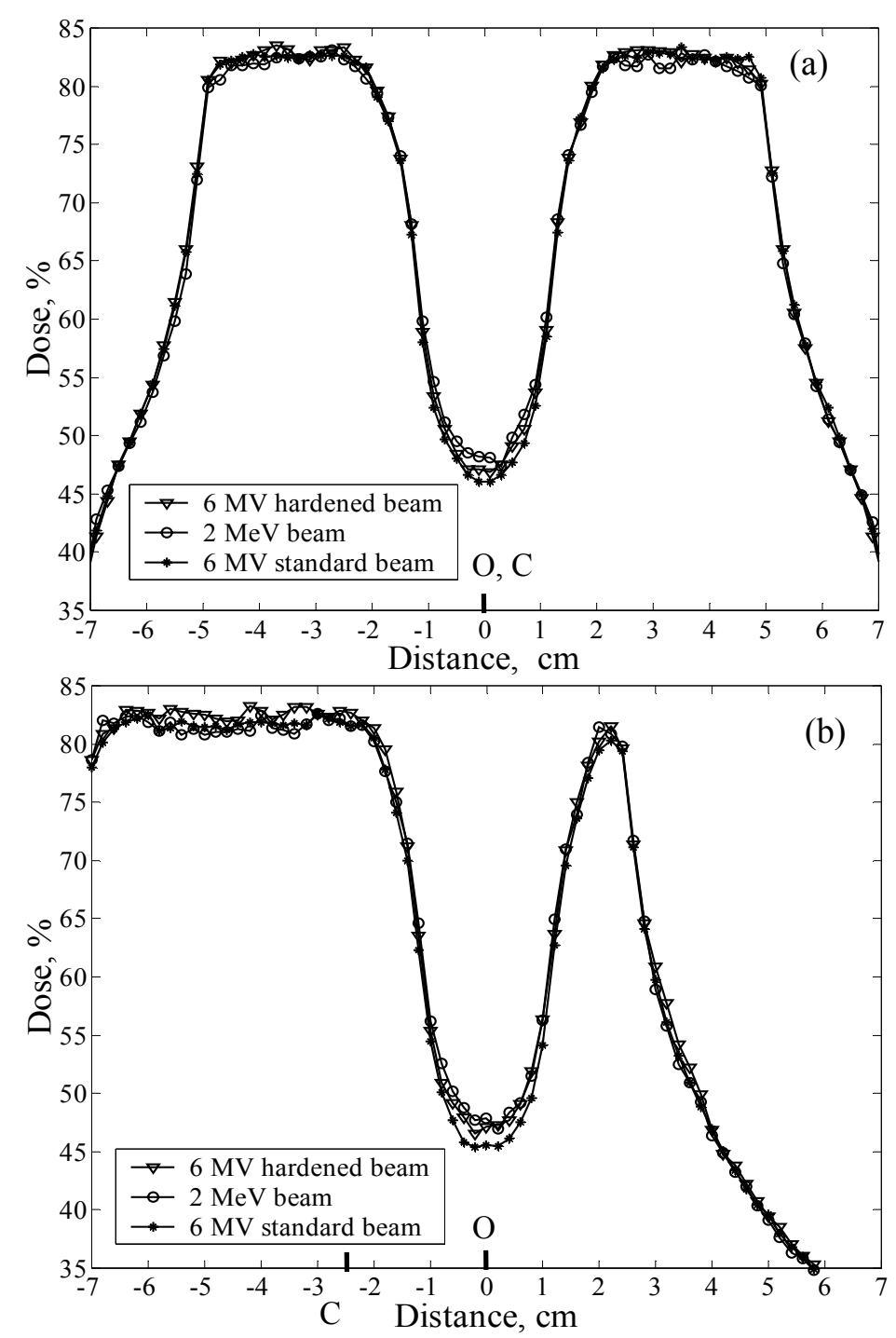

FIGURE 5-10. Central dose profiles of rotational irradiation received using the beam modifying devices of the geometry listed in Table 5-1. Monoenergetic $2 \mathrm{MeV}$ and polyenergetic hardened and ordinary $6 \mathrm{MV}$ beams (ELECTA SL75) were used for simulation studies. The dose profiles are shown for $\mathrm{CO}=0 \mathrm{~cm}$ (a) and $\mathrm{CO}=2.5 \mathrm{~cm}(\mathrm{~b})$.

The intensity modulation with the same beam modifying devices resulted in differing dose distributions. The dose values in the OAR region are slightly higher for the hardened $6 \mathrm{MV}$ beam than for the standard ELECTA SL75 $6 \mathrm{MV}$ beam. For $2 \mathrm{MeV}$ beam the dose at the OAR is the highest. The weighted mean linear attenuation coefficients in lead are $0.5344 \mathrm{~cm}^{-}$ $1,0.5666 \mathrm{~cm}^{-1}$ and $0.5142 \mathrm{~cm}^{-1}$ for polyenergetic $6 \mathrm{MV}$ hardened, $6 \mathrm{MV}$ standard and monoenergetic $2 \mathrm{MeV}$ beams, respectively. So, $2 \mathrm{MeV}$ beam appears to be more penetrating through the protector and it deposits higher dose into the water phantom. In the case, in which $\mathrm{CO}=0$, the dose value at the center of the OAR region for $2 \mathrm{MeV}$ beam is $48.2 \%$ (see Fig. 510(a)) and is by $2.4 \%$ higher than of the hardened $6 \mathrm{MV}$ and by $4.4 \%$ higher than of the standard $6 \mathrm{MV}$ beam. For $\mathrm{CO}=2.5 \mathrm{~cm}$, the dose value at the center of OAR for $2 \mathrm{MeV}$ is 
47.5\% (see Fig. 5-10(b)) and is by $1.1 \%$ and $4.2 \%$ higher than of $6 \mathrm{MV}$ hardened and standard beams, respectively. The difference in the dose profiles is observed at the regions attenuated by the iroco shapers, as well as. Here, the highest dose hardened $6 \mathrm{MV}$ beam has, then $6 \mathrm{MV}$ standard follow. A $2 \mathrm{MeV}$ beam has the lowest dose in the plateau region. This is in accordance to the weighted mean linear attenuation coefficients for iroco wood. For these beams the values are $0.0308 \mathrm{~cm}^{-1}, 0.0322 \mathrm{~cm}^{-1}$ and $0.0339 \mathrm{~cm}^{-1}$, correspondingly.

\subsubsection{Assessment of the shapers' parameters calculation methods}

\subsubsection{1st method "from diameter to material"}

According to the arguments presented in sect. 5.2.5.2.1, the shapers of $9.5 \mathrm{~cm}$ in diameter may be made of iroco wood, as well as the shapers of $6.5 \mathrm{~cm}$ in diameter may be made of polystyrene to suit the defined requirements. Dose distributions obtained in simulations of irradiation of two configurations of the beam modifying devices are shown in Figs. 5-11(a) and 5-11(b) for the two irradiation setups, respectively. As demonstrated in these figures, the iroco shapers provide slightly higher doses within the irradiated area, compared to the polystyrene shapers (average doses in the plateau regions are $82.6 \%$ versus $81.2 \%$ for $\mathrm{CO}=0 \mathrm{~cm}$ and $82.5 \%$ versus $80.8 \%$ for $\mathrm{CO}=2.5 \mathrm{~cm}$ ).

\subsubsection{2nd method "from material to diameter"}

Figures 5-12(a) to 5-12(d) present the central dose profiles acquired after the use of shapers, which were calculated as described in Chapter 4, sect. 4.3.2.2. The initial dose profiles, acquired by use of a beam with a protector of $1.6 \mathrm{~cm}$ in diameter inserted in it, are also shown in the figures. Based on these profiles, the features of the desired dose distributions were extracted and used in shapers' parameters calculations.

A statistical evaluation of the resultant dose distributions is summarized in the Table 56, where the defined and average acquired dose values at the PTV are presented as well as standard deviations and relative errors of the simulated dose distributions. The achieved dose distributions have a good agreement with the desired dose distributions, as the statistical results affirm. A slightly worse agreement is for the forth experiment in which polystyrene shapers were used. 

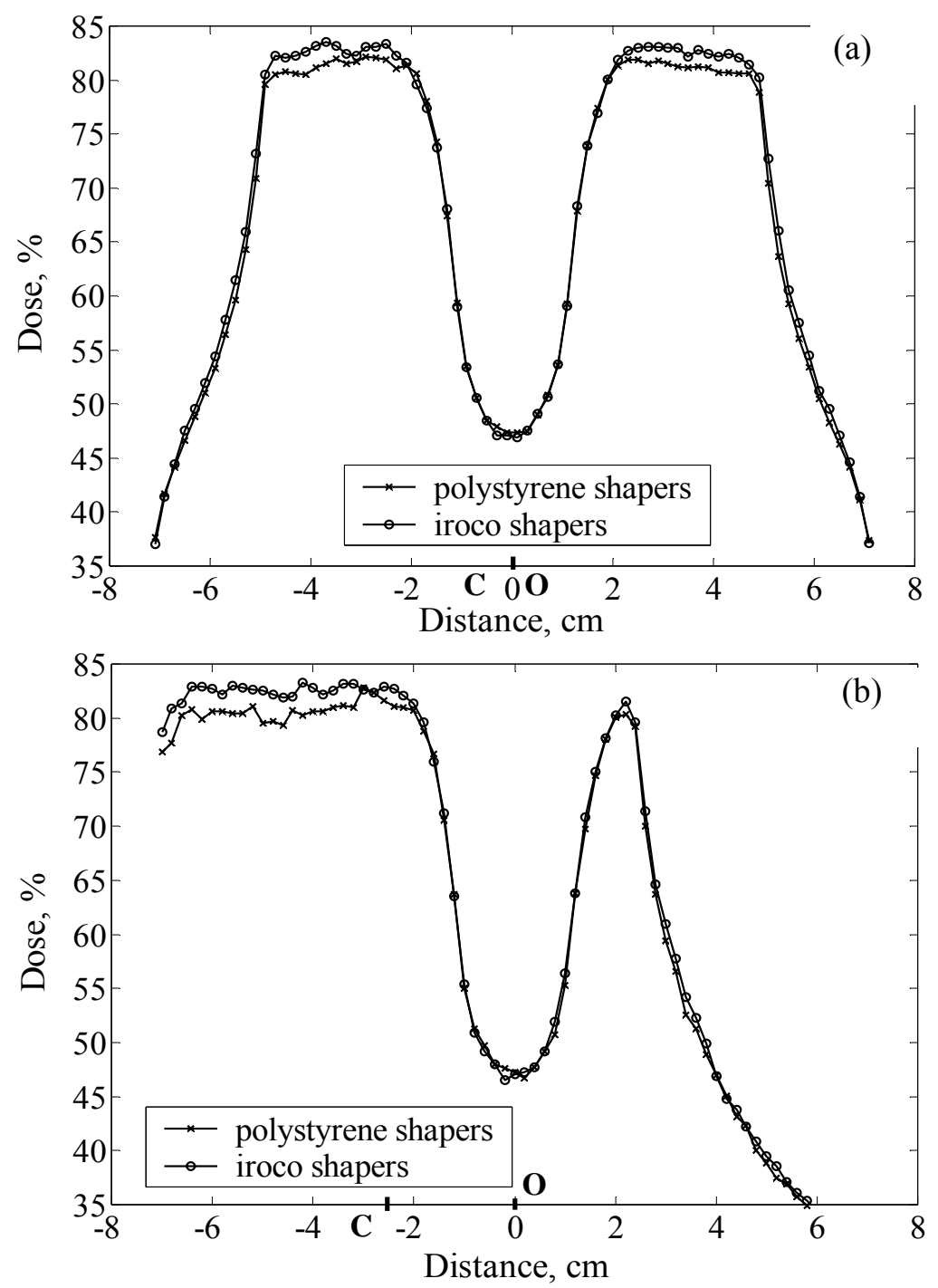

FIGURE 5-11. Comparison of a beam shaping with two configurations of the beam modifying devices in rotational radiotherapy. Both configurations contain the same protector and they differ with the geometry and materials of shapers. (a) represents the transverse dose profiles at the center of the cylindrical neck model for the irradiation setup with the isocenter coinciding with the center of the OAR and (b) with the centers shifted $2.5 \mathrm{~cm}$ relative to each other. A hardened beam of $6 \mathrm{MV}$ was used for the simulations. 

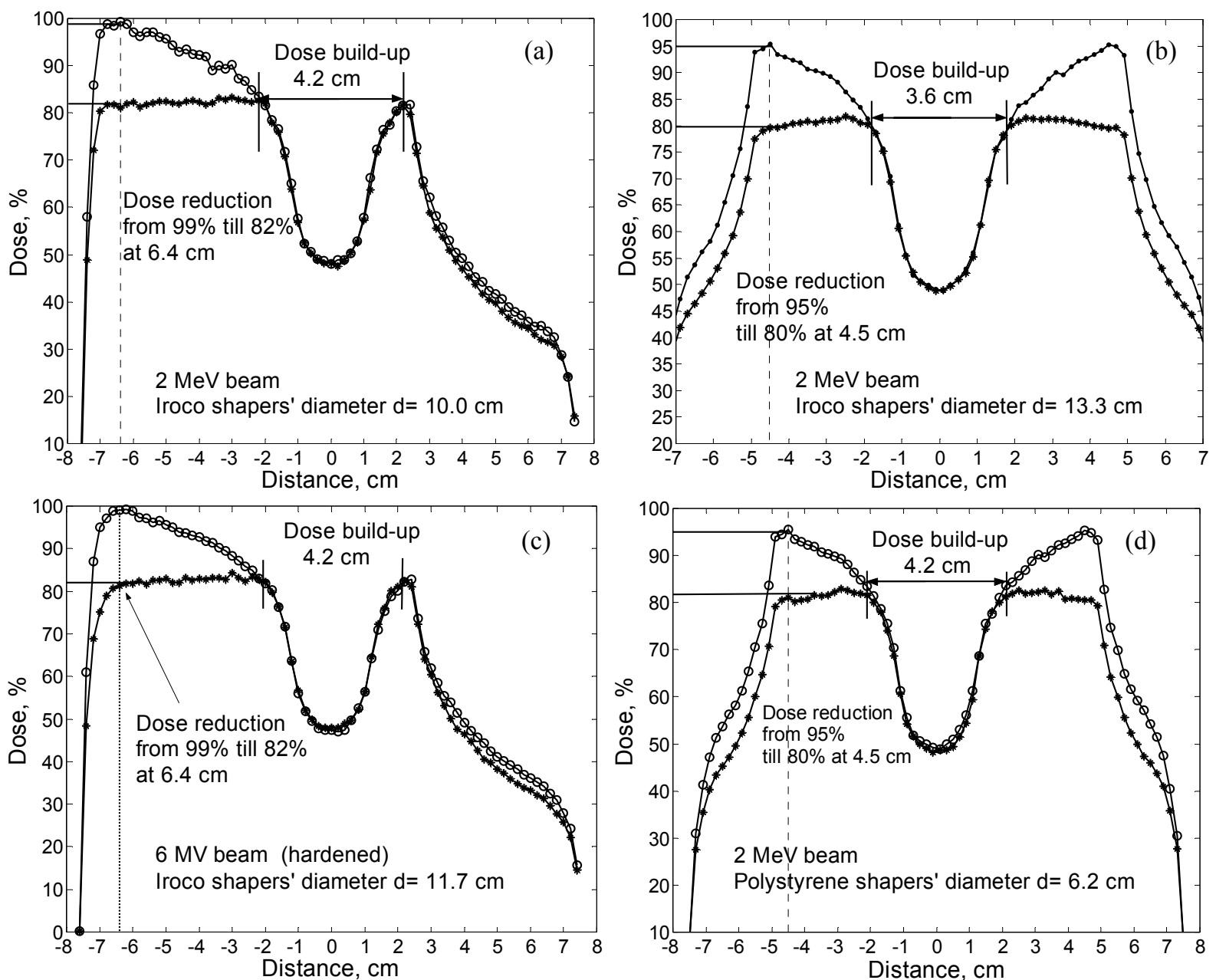

FIGURE 5-12. Dose distributions received by intensity modulation by means of beam modifying devices: lead protector of $1.6 \mathrm{~cm}$ in diameter and beam shapers with parameters, given in Table 5-4. Studies numbered in the table from 1 to 4 correspond to figures (a) to (d).

TABLE 5-6. The achieved PTV doses after using the beam shapers.

\begin{tabular}{lcccc}
\hline \hline & Experiment 1 & Experiment 2 & Experiment 3 & Experiment 4 \\
\hline Desired PTV dose, \% & 82 & 80 & 82 & 82 \\
Achieved PTV dose & 82.2 & 80.5 & 82.1 & 81.2 \\
(average value), \% & & & & \\
Standard deviation, \% & 0.68 & 0.88 & 0.37 & 1.12 \\
Relative error, \% & 0.8 & 1.1 & 0.5 & 1.4 \\
\hline \hline
\end{tabular}




\subsubsection{Application study with the voxel-based neck model}

The degree of conformity and homogeneity of the computed dose distribution in the voxel-based neck model is demonstrated in Fig. 5-13. The isodose curves are expressed as percentage of the dose at the isocenter. The calculated isodose curves superimposed on the sagittal cross-section, passing through the center of the spinal cord, are shown in Fig. 5-13(a). The isodose distributions for the coronal sections, taken at the isocenter and at the center of the OAR respectively, are shown in Figs. 5-13(b) and 5-13(c). Finally, the isodose distribution for the transverse cross-section, passing through the isocenter, is depicted in Fig. $5-13(d)$. Dose values at the OAR are in the range of $55 \%$ to $74 \%$ of the dose at the isocenter.
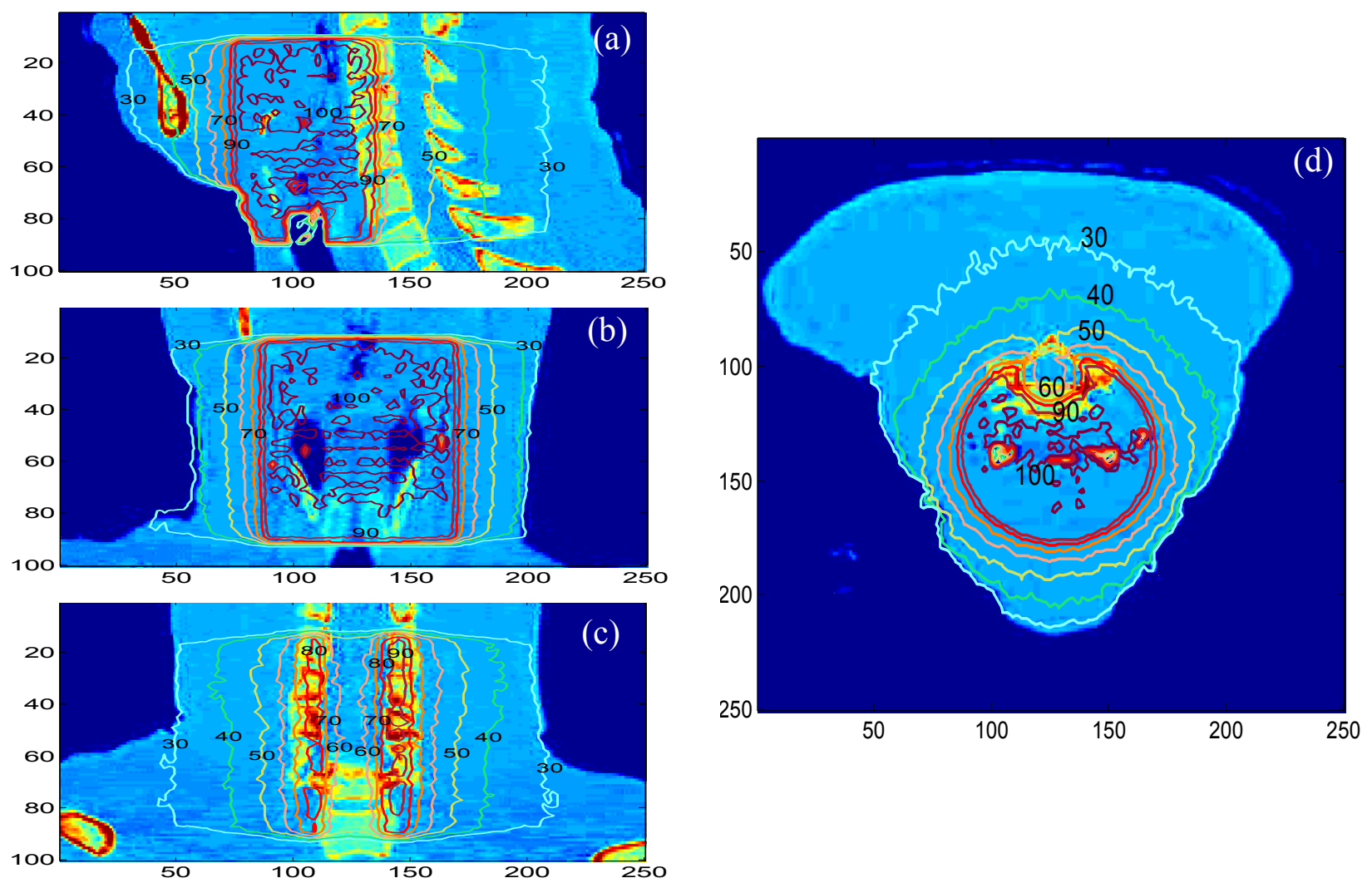

FIGURE 5-13. Isodose curves, superimposed on sagittal (a), coronal (b) and (c) and transverse (d) cross sections of the voxel-based phantom. The cross sections (a) and (c) are shown at the center of the spinal cord, while (b) and (d) represent cross sections taken at the isocenter. Intensity modulation for rotational radiotherapy is performed by means of the beam modifying devices. The dose curves are expressed as a percentage of the dose at the isocenter. A hardened beam of $6 \mathrm{MV}$ was used for simulations. 


\subsection{DISCUSSION AND CONCLUSIONS}

The verification of the developed software tool for the calculation of the parameters and the location of the beam modifying devices gave quite satisfactory results. The experimental and simulated data were found to be in good agreement, with discrepancies not higher than $3 \%$. The larger differences between experimental and simulated data are at the regions with high dose gradient: just outside the OAR and at the field edges. According to the verification results, the software tool developed was proven to be a reliable tool for studies of beam modulation with beam modifying devices.

The studies of the intensity modulation showed that the lead cylinder of $1.6 \mathrm{~cm}$ in a diameter, introduced in the beam, results to a significant dose reduction. The dose has a variation across the protected region with the minimal dose at the center of the OAR. The dose variation is also characteristic for static narrow blocks of large thickness (Ezzell et al. 1987). The dose reduction in the studied case was of $57 \%$ at the center of the OAR till $77.5 \%$ at the periphery of the OAR. Usually for head and neck tumors treatment doses in the range of 70 Gy are needed, whereas the spinal cord dose should not exceed 45 to 50 Gy (Martel et al. 1997, Brantengeier et al. 2000), which is $64 \%$ to $71 \%$ of the curative dose. It is important to keep the dose within the whole OAR lower than the tolerance level. In the case studied, the dose, however, increases rapidly at the border of the cylindrical OAR, and within the outer annulus of $1 \mathrm{~mm}$ is beyond the tolerance level (it increases from $71 \%$ to $77.5 \%$ ). The attenuation of the beam due to inserted protector for small structures is limited as a protector of small diameter has to be used and may be not sufficient. However, use of higher density material, for instance tungsten or gold instead of lead, can increase the attenuation and, thus, protection. Alternatively, the degree of protection can be increased by locating the protector further from the source and using a protector of a larger size. In this case, the skin sparing effect must be taken into account due to excessive beam contamination with secondary electrons and scattered photons. An air gap of $15-20 \mathrm{~cm}$ between the scatterer and the skin is considered adequate to keep the skin dose to the acceptable level (Khan 1994).

The dose of the OAR remains the same in the simulations with a single protector and with the whole assembly of the beam modifying devices. Thus, the protection of the OAR is not affected by the shapers. The dose outside the attenuated region increases non-uniformly. The beam must be increasingly filtered as the distance from the center of the OAR increases and circular shapers were used for this purpose. Using the beam shapers, a uniform dose outside the protected region is obtained, as the simulation results depicted in Figs. 5-7 and 5-8 
show. Unfortunately, the uniform dose is achieved at the expense of the reduction of the dose by shapers at the PTV. The ratio of the dose at the OAR to the dose at the PTV, however, is important and it's essential to keep it not higher than recommended, for example for head and neck cancers it must not be higher than $50 \mathrm{~Gy} / 70 \mathrm{~Gy}=0.71$. It should be also noted, that the relative position of the isocenter with respect to the center of the OAR is of negligible importance (Proimos 1961). The other positive feature of the shapers use is that the dose gradient at the borders of the field is steeper, thus the unnecessary dose to the healthy tissue is reduced.

The beam modifying devices used for intensity modulation for cylindrical planning target volume near a single cylindrical OAR showed promising results. The presented concept can be easily extended to include other concave or convex shapes of OAR (Danciu 2001). If protection for multiple OARs has to be provided, a protector for every single OAR separately has to be calculated, and afterwards all of them have to be incorporated in the common system. The methodology of shapers modeling is more complicated.

Moreover, it was interesting to study how much the dose distribution is affected by the beam characteristics. The results of this study, in which $2 \mathrm{MeV}$ and ELECTA-SL75 $6 \mathrm{MV}$ standard and hardened beams were involved, showed, that intensity modulation with the same beam modifying devices resulted in slightly differing dose distributions. It was expected, because though close but, nevertheless, different irradiation beams' qualities and energies were exploited. It should be noted, though it was not the objective of our studies, that the ratio of the dose at the center of the OAR to the average dose value in the PTV for $2 \mathrm{MV}$ beam is larger than for both $6 \mathrm{MV}$ beams, so it shows worse performance. The dose outside the OAR remains uniform for all irradiation beams. This study illustrated also that a replacement of a polyenergetic beam by a monoenergetic one of its average energy is not justified; however, simulations involving a monoenergetic beam require less photon histories for the same statistical noise. So, it can be used for faster evaluation of the results.

The shapers' parameters were calculated in this work in two ways. The first: "from diameter to material", that is, to fix the diameter of the shaper, and afterwards to determine its possible material. Simulation of the rotational irradiation with the beam modulated with the same protector, but shapers made of different materials, determined accordingly to this method, demonstrated a similar pattern of dose distributions, with a slightly higher dose in the PTV when using iroco shapers. More uniform dose in the PTV for the case of the iroco shapers was also noticed. The diameter of the polystyrene protector seems to be 
overestimated. It has, however, the disadvantage of limited number of materials, suitable for shapers design. Also, the method requires an initial guess of the shaper's diameter and generally is not very precise as a number of the diameters can lead to values that are close to the linear attenuation coefficient of some material.

The second method of shapers' parameters calculations is "from material to diameter". It is more flexible and provides acceptable approach to the shapers' parameters calculation. The verification of this method showed that it is sufficiently precise and can be successively used for shapers design. A tendency of a small overestimation of the diameter is noticed. For monoenergetic beams the linear attenuation coefficient is known exactly and the desired dose distributions approaches closer to the desired one. For a polyenergetic beam for which weighted mean linear attenuation coefficient is involved in the calculations, the correspondence to the defined dose distribution is slightly worse, because $\bar{\mu}$ can not reflect comprehensively the properties of a polyenergetic beam. Moreover, the beam hardening and the scatter effects were not included.

The simulation of beam modifying devices with the voxel-based phantom resulted in acceptable uniform irradiation in the target volume without exceeding the dose tolerances for the nearby critical structures, such as the spinal cord. The spinal cord doses were limited to $70 \%$ at the dose at the isocenter, however, a dose increase to $74 \%$ was observed in the very proximity of the vertebrae.

Conceptual studies have shown that design of concave dose distributions with simultaneous shielding of the organ at risk can be achieved by means of beam modifying devices. In cases requiring spinal cord protection, simulation studies showed a high degree of conformity.

Advantages of using this technique include comprehensive and adequate protection at every gantry angle, uniform dose in PTV, and use of inexpensive materials. More detailed studies of the effect of the protector parameters on the dose distribution are presented in the next chapter. 


\section{STUDIES ON THE EFFECT OF PROTECTORS ON DOSE DISTRIBUTIONS IN A HOMOGENEOUS PHANTOM}

\subsection{INTRODUCTION}

Adequate radiation doses sometimes are difficult to attain because of a tumor proximity to the Organ At Risk (OAR), like in the case of head and neck tumors with regional metastases, due to proximity of the lymph nodes to the spinal cord. Nevertheless, adequate protection of the OAR and uniform dose at the target volume has to be provided. Cylindrical attenuator inserted in the treatment beam can effectively be used for a protection of the spinal cord in the case of rotational therapy (Proimos 1961, Danciu 2001, Jena et al. 2004, Ivanova et al. 2006). The idea of the protection is simple, evident and elegant. If the protector stays parallel to the OAR during the whole treatment and is of appropriate size, its cast shadow coincides with the OAR at every gantry angle, thus keeping low dose for the OAR. However, except the geometrical considerations of the protector design, it is important to study some physical characteristics of the dose distributions obtained by using a protector inserted in the rotational treatment beam. The effect of the protector's parameters and location on the dose 
distribution has a complicated character. At photon energies, where the Compton effect is dominant interaction process, the beam-modifying filter also produces scattered radiations; secondary electrons and scattered photons thus impinge on the patient. The electrons compromise the skin sparing characteristics of the beam, while the presence of scattered photons may complicate the design of beam modifiers.

In partially attenuated beams the dose on the central axis below the absorber depends on the standard percentage depth dose (PDD) parameters: depth of measurements, field size, source-to-surface distance, and beam energy. In addition, it also depends on the attenuator thickness and material, the distance from the phantom surface and the attenuated field size, which are all characteristic of the attenuator (El-Khatib et al. 1988). If we consider separately the primary and scattered beam components, it can be noted that the transmission of the primary (narrow) beam through an absorber depends upon the beam energy, type and the thickness of the absorber. In addition to a beam energy and absorber material, the scatter component for a broad beam also depends on the field size, thickness and shape of the absorber, location of the absorber with respect to the source, and the point of calculation (Islam and Van Dyk 1995).

In this chapter the effect of beam attenuators inserted in the beam on the dose distribution was studied. Measured beam attenuation by a number of materials, new at the market and traditionally used in radiation therapy for a protection of vital organs, and the corresponding effective linear attenuation coefficient were compared with the results of simulations. The purpose was twofold: (1) to study the attenuation properties of the materials and (2) to verify some aspects of the Monte Carlo Radiotherapy Simulator (MCRTS), comparing the results of simulations with the experimental measurements of the beam attenuation by different materials. After the MCRTS verification, more complicated simulation studies that can not easily be implemented experimentally were performed. Simulation studies of the effect of the cylindrical protector's diameter, its material and distance between the protector and a phantom surface on the dose distribution in a homogeneous phantom were carried out. The quality of the received dose distributions was evaluated in terms of dose at the center of the OAR $\left(D_{c}\right)$, phantom surface dose, the ratio of the dose at the center of OAR to the maximal dose at the Planning Target Volume (PTV) $\left(\mathrm{D}_{\mathrm{c}} / \mathrm{D}_{\max }\right)$ and the dose increase (gradient) outside the OAR.

All these characteristic parameters of the dose distribution are clinically important, especially a surface dose. Treatment with high energy beam has an advantage of skin sparing. 
Studies of the influence of the attenuator's parameters and materials upon the surface dose will help to preserve the skin sparing effect. Khan (1994) explains that by placing absorber in the beam, for $10 \mathrm{MV}$ x-rays the surface dose may be increased as much as $50 \%$ for low atomic number $(\mathrm{Z})$ materials and as much as $30 \%$ for high $\mathrm{Z}$ materials compared to open beam. It was shown also that the Lipowitz metal tissue compensators increase the relative surface dose in cases of increased field size, decreased source-to-surface distances, use of low Z blocking tray, and increased energy from 6 to 24 MV x-ray beams (Cardarelli et al. 1991).

Another important feature of the dose distributions in rotational therapy with partially attenuated fields is the dose increase immediately outside the OAR (dose build-up region). For some treatments, such as in the case of vertebral metastases, high dose gradient in dose build-up region is of big importance.

\subsection{THEORETICAL BACKGROUND}

The general theory of gamma ray absorption has been clearly outlined in a paper by Davisson and Evans (1952). The basis of absorption measurements is exponential attenuation. For monoenergetic radiation and narrow beam geometry this is expressed by

$$
\frac{\mathrm{I}}{\mathrm{I}_{0}}=\mathrm{e}^{-\mu \mathrm{x}}
$$

where I is the intensity (energy fluence) of radiation after positioning of absorber in the beam and $\mathrm{I}_{0}$ is the initial intensity, $\mu$ is the linear attenuation coefficient in $\mathrm{cm}^{-1}$, and $\mathrm{x}$ is the absorber thickness in $\mathrm{cm}$.

The effects of first scatter can be calculated by using the expressions as derived by Davisson and Evans (1952) (Eq. 72). The ratio of scatter-to-primary intensity is given by

$$
\mathrm{S} / \mathrm{I}=\mathrm{N}_{\mathrm{e}} \mathrm{X}_{0} \sigma_{0}
$$

where $\mathrm{S}$ represents the first scatter intensity, $\mathrm{N}_{\mathrm{e}}$ is the number of electrons $/ \mathrm{cm}^{3}$ in the absorber, $\mathrm{x}_{0}$ is the absorber thickness in $\mathrm{cm}$, and $\sigma_{0}$ is the integrated cross section for the energy of the photons scattered between $0^{\circ}$ and the maximum scattering angle and is given in $\mathrm{cm}^{2}$. This latter quantity is a complicated function of incident energy and the maximum scattering angle (Eq. 26, in Davisson and Evans, 1952).

To obtain the fraction of the $\gamma$-ray energy scattered in a given direction $\theta$, Klein and Nishina have carried out a quantum-mechanical treatment of the problem and showed that the 
differential cross-section per unit solid angle is given by the product of the Thomson scattering expression and the factor $\mathrm{F}_{\mathrm{KN}}$ (Davisson and Evans 1952, Johns and Cunningham 1983c):

$$
\frac{\mathrm{d} \sigma}{\mathrm{d} \Omega}=\frac{\mathrm{d} \sigma_{0}}{\mathrm{~d} \Omega} \cdot \mathrm{F}_{\mathrm{KN}}=\frac{\mathrm{r}_{0}^{2}}{2}\left(1+\cos ^{2} \theta\right) \cdot \mathrm{F}_{\mathrm{KN}}
$$

where

$$
\mathrm{F}_{\mathrm{KN}}=\left\{\frac{1}{1+\alpha(1-\cos \theta)}\right\}^{2}\left\{1+\frac{\alpha^{2}(1-\cos \theta)^{2}}{[1+\alpha(1-\cos \theta)]\left(1+\cos ^{2} \theta\right)}\right\},
$$

$\mathrm{r}_{0}$ is the classical electron radius and is given by $\mathrm{e}^{2} / \mathrm{m}_{0} \mathrm{c}^{2}$, the parameter $\alpha$ is the ratio of photon energy to the electron rest mass energy $\left(\mathrm{h} v / \mathrm{m}_{0} \mathrm{c}^{2}\right)$ and $\theta$ is the photon scattering angle.

\subsection{MATERIALS AND METHODS}

\subsubsection{Choice of the materials appropriate for beam modifiers manufacturing}

A range of related literature was reviewed in order to identify the best materials suitable for manufacturing of beam modifiers for radiation shielding purposes. Both the density of a beam modifier and its maximum thickness limit the intensity modulation range achievable. For example, for a tin granule compensator of $5 \mathrm{~cm}$ in thickness the max intensity modulation range achievable is approximately $100 \%$ to $38 \%$ and $45 \%$ of the open field for $6 \mathrm{MV}$ and 15 $\mathrm{MV}$, respectively. With the came compensator design coarse tungsten powder offers a much larger intensity modulation range: $100 \%$ to $18 \%$ and $20 \%$ for $6 \mathrm{MV}$ and $15 \mathrm{MV}$, respectively. Tungsten powder has an effective density of more than $10 \mathrm{~g} / \mathrm{cm}^{3}$ compared to $4.6 \mathrm{~g} / \mathrm{cm}^{3}$ for tin granules (Chang et al. 2004).

Two kinds of beam modifiers are used in radiation therapy: blocks and compensators. Blocks are usually made of high-density material of big thickness, as it is described in chapter 2, section 2.2.2., and are used for a shielding of vital organs. Compensators are used to achieve various goals (refer to chapter 2) and therefore materials used for their manufacturing can be of different densities. The range of densities can vary from the tissue's density till the higher densities, like of lead. However, denser materials react with greater sensitivity to the changes in a material thickness and, thus, the inaccuracies in a manufacturing process or a calculation method are more obvious. The number of materials used to form compensators is presented in Table 6-1. 
TABLE 6-1. Materials that are used as beam modifiers in radiation therapy, their densities and narrow beam linear attenuation coefficients.

\begin{tabular}{|c|c|c|c|}
\hline & Material & Density, $\mathbf{g} / \mathrm{cm}^{3}$ & $\begin{array}{l}\text { Linear attenuation } \\
\text { coefficient }(6 \mathrm{MV}), \mathrm{cm}^{-1}\end{array}$ \\
\hline \multirow{17}{*}{ 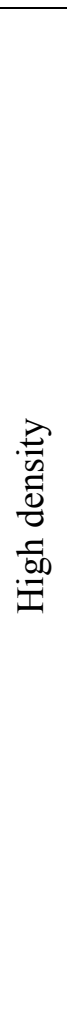 } & Gold (Danciu 2001) & 19.3 & 0.9600 \\
\hline & Tungsten (Danciu 2001) & 17.1 & 0.8450 \\
\hline & Tungsten (Tajiri et al. 2004) & N/A (19.3)* & 0.816 \\
\hline & Rad-block (Kanebo) (Tajiri et al. 2004) & 12 & 0.553 \\
\hline & $\begin{array}{l}\text { Tungsten- dominant blocks (Kanebo) (Nakadawa } \\
\text { et al. 2005) }\end{array}$ & 12 & 0.518 \\
\hline & Lead (Van Dyk 1986) & 11.36 & 0.5164 \\
\hline & Lead (Danciu 2001) & 11.35 & 0.5640 \\
\hline & Lead (Tajiri et al. 2004) & $\mathrm{N} / \mathrm{A}(11.35)^{*}$ & 0.519 \\
\hline & Lead (du Plessis et al. 2003) & 11.34 & 0.571 \\
\hline & Heavy alloy granules (Yoda et al. 2003) & 10.1 & 0.515 \\
\hline & Tungsten powder (Chang et al. 2004) & $>10$ & 0.430 \\
\hline & $\begin{array}{l}\text { Cerrobend(Lipowitz's metal: } 13.3 \% \text { tin, } 59 \% \\
\text { bismuth, } 26.7 \% \text { lead, } 10 \% \text { cadmium) (Danciu }\end{array}$ & & \\
\hline & 2001) & 10 & 0.4662 \\
\hline & Lipowitz's metal (El-Khatib et al. 1987) & 9.63 & 0.4880 \\
\hline & Lipowitz's metal (Tajiri et al. 2004) & N/A & 0.490 \\
\hline & $\begin{array}{l}\text { Mixture of tungsten powder ( } 50 \%) \text {, silicon rubber } \\
(35 \%) \text {, and paraffin }(15 \%) \text { (Xu et al. } 2002)\end{array}$ & N/A & 0.409 \\
\hline & Brass (du Plessis et al. 2003) & 8.47 & 0.386 \\
\hline \multirow{11}{*}{ 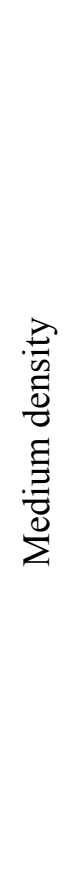 } & Tin granules (Chang et al. 2004) & 4.6 & 0.217 \\
\hline & $\begin{array}{l}\text { Granulate stainless steel (van Santvoort et al. } \\
\text { 1995) }\end{array}$ & $\sim 4.5$ & $\sim 0.218$ \\
\hline & $\begin{array}{l}\text { Refined gypsum }+ \text { stainless-steel (steel } 67 \% \text { by } \\
\text { weight, water/gypsum ratio is } 13 / 100 \text { ) (Weeks et } \\
\text { al.1988) }\end{array}$ & 3.4 & 0.139 \\
\hline & Polyethylene- lead (Mageras et al. 1990) & 2.9 & 0.132 \\
\hline & $\begin{array}{l}\text { Refined gypsum }+ \text { iron (iron } 45 \% \text { by weight, } \\
\text { water/gypsum ratio 13/100) (Weeks et al.1988) }\end{array}$ & 2.7 & 0.118 \\
\hline & Aluminum (du Plessis et al. 2003) & 2.68 & 0.131 \\
\hline & $\begin{array}{l}\text { Cement based material Densitop } \mathrm{T} 2{ }^{\circledR} \text { (Thomsen et } \\
\text { al. 2002) }\end{array}$ & 2.677 & similar to aluminum \\
\hline & $\begin{array}{l}\text { Special, refined gypsum (water/gypsum ratio } \\
\text { 20/100) (Weeks et al. 1988) }\end{array}$ & $\sim 2$ & 0.092 \\
\hline & Thermo-Shield $^{\mathrm{TM}}$ (Paliwal et al. 1998) & 1.66 & 0.205 \\
\hline & $\begin{array}{l}\text { Wax-gypsum ( } 40 \% \text { dental wax }+60 \% \text { gypsum) } \\
\text { (du Plessis et al. 2005) }\end{array}$ & 1.48 & 0.0685 \\
\hline & Wax (du Plessis et al. 2003) & 0.9 & 0.037 \\
\hline
\end{tabular}

* Hubbell J.H. and Seltzer S.M. (2004)

Note: Tissue-equivalent substances like wax or plastic were not comprehensively included

Metal granules and powders are also used as filling material in compensators design (Yoda et al. 2003, Xu et al. 2002, van Santvoort et al. 1995). They do have some advantages 
compared to the solid form (Chang et al. 2004). However, granules, for example, only have $52 \%$ of the linear attenuation coefficient of the solid form, if granules are identical spheres. One way to increase the packing density is to mix granules of different sizes and shapes.

It should be also mentioned here that a new material, called Rad-block, appeared in the field of radiation protection recently. It is composite and is produced of tungsten powder and polymer. It has high attenuation coefficient that is higher than of Cerrobend and lead. Except advantageous shielding properties, it is easy to fabricate and friendly to environment (Tajiri et al. 2004).

\subsubsection{Measurements of beam attenuation by different materials}

\subsubsection{The attenuators}

The attenuators used in the experiments consisted of some materials commonly used for protection in radiotherapy, such as lead, cerrobend (or Lipowitz's metal) and brass, as well as of three new materials for radiation shielding: Ecomass ${ }^{\circledR}$ compound 1700TU96 (Ecomass Technologies), Gravi-Tech ${ }^{\mathrm{TM}}$ GRV-AS-110-W (PolyOne Corporation) and Technon®/Poly (Tungsten Heavy Powder, Inc.). The new materials are polymer-metal composites analogous to Rad-block. These composites are based on different polymers (nylon 12, acrylonitrile butadiene styrene (ABS) and polyurethane (PU) based resin, accordingly) filled with tungsten powder to achieve high specific gravity compounds. Some physical properties of these materials and thicknesses of the slabs used in the measurements are summarized in Table 6-2. Ecomass samples used in our experiments were donated by Ecomass Technologies and received from the manufacturer in a form of molded plaques. Gravi-Tech samples, donated by PolyOne Corporation, initially were in the shape of hollow cylinders and an attempt was made to reshape the samples in a planar plaques. The cylinders were cut along the diameter and subjected to warming by a hot air gun and immediate pressing. Technon/Poly plaques were produced manually from tungsten Technon ${ }^{\circledR}$ powder and corresponding polymer, provided in the kit by the manufacturer. Tungsten powder was added in half amounts to the polymer while mixing in the ratio 96:4. The molds made of Plexiglas were filled with the received heavy paste. For enhanced physical properties the plaques were placed in the oven and kept at $66^{\circ} \mathrm{C}$ during 4 hours for post curing. 
TABLE 6-2. Materials used in beam attenuation measurements. The table presents materials' density, atomic number, composition and the thicknesses of the slabs used.

\begin{tabular}{|c|c|c|c|c|c|}
\hline & Material & Density, $\mathrm{g} / \mathrm{cm}^{3}$ & $\overline{Z Z}$ & $\begin{array}{l}\text { Composition, } \\
\text { (wt. \%) }\end{array}$ & $\begin{array}{l}\text { Slabs thicknesses, } \mathrm{mm} \\
( \pm 0.2)\end{array}$ \\
\hline 1 & Lead & $11.35^{*}$ & 79 & $\mathrm{~Pb}(100)$ & $4 \times 2.3 ; 3 \times 2.2$ \\
\hline 2 & Cerrobend & $9.63 * *$ & $75.3^{\dagger}$ & $\begin{array}{l}\mathrm{Sn}: \mathrm{Bi}: \mathrm{Pb}: \mathrm{Cd} \\
(13.3: 50: 26.7: 10)\end{array}$ & $\begin{array}{l}5.3,5.5,5.4 \\
6.9,7.3,8.9\end{array}$ \\
\hline 3 & Brass & $8.9^{*}$ & $29.4^{\dagger}$ & $\mathrm{Cu}: \mathrm{Zn}(66: 34)$ & $4 \times 5.0 ; 2 \times 6.3$ \\
\hline 4 & Ecomass & $(10.73 \pm 0.53)$ & $72.8^{\dagger}$ & W: nylon $12^{\ddagger}(97: 3)$ & $2 \times 6.6 ; 2 \times 6.7$ \\
\hline 5 & Gravi-Tech & $(8.15 \pm 0.23)$ & $72.2^{\dagger}$ & W:ABS ${ }^{\ddagger}(96: 4)$ & $\begin{array}{l}1 \times 2.1 ; 1 \times 2.2 ; 2 \times 2.3 ; \\
3 \times 2.4 ; 2 \times 2.5\end{array}$ \\
\hline 6 & Technon/Poly & $(8.17 \pm 0.15)$ & $72.1^{\dagger}$ & $\begin{array}{l}\text { W:[PU resin }+ \text { PU } \\
\text { curing agent }]^{\ddagger}(96: 4)\end{array}$ & $\begin{array}{l}3.7,4.0,4.0,4.1,4.2, \\
4.4\end{array}$ \\
\hline
\end{tabular}

* Danciu (2001)

**El-Khatib et al. (1987), Cardarelli et al. (1991).

${ }^{\top}$ The effective atomic number for compounds was calculated using $\mathrm{Z}^{3.5}$ dependence (Johnes and Cunningham 1983).

\$Chemical formulas:

- nylon $12\left(\mathrm{C}_{12} \mathrm{H}_{23} \mathrm{ON}\right)$;

- $\operatorname{ABS}\left(\mathrm{C}_{15} \mathrm{H}_{17} \mathrm{~N}\right)$;

- $\quad[\mathrm{PU}$ resin + PU curing agent] was modeled consisting of $70 \%$ of methylene bis(4phenylisocuanate) $\left(\mathrm{C}_{15} \mathrm{H}_{10} \mathrm{~N}_{2} \mathrm{O}_{2}\right)$ and $30 \%$ of polyurethane $\left(\mathrm{C}_{27} \mathrm{H}_{36} \mathrm{~N}_{2} \mathrm{O}_{10}\right)$.

The density of the Technon/Poly material was expected to be $10.5 \mathrm{~g} / \mathrm{cm}^{3}$ according to the manual, nevertheless, the measured density appeared to be $8.17 \mathrm{~g} / \mathrm{cm}^{3}$ that can be explained by a possible porous structure of the compound acquired during the manufacturing procedure. The measured density of Gravi-Tech compound was also lower than declared by the manufacturer. The most probable reason can be the additional processing of the material that was performed in order to achieve planar samples. The densities of the polymer-metal composites were found by weighting the samples and determine the volume by the water displacement method.

\subsubsection{The experimental setup}

The measurements were performed for narrow beam geometries. The primary beam transmission in the materials was measured using the ionization chamber (PTW M23332, $0.3 \mathrm{~cm}^{3}$ ) connected to the electrometer (PTW DI4). The chamber was placed in a polymethyl methacrylate (PMMA) phantom $\left(\rho=1.18 \mathrm{~g} / \mathrm{cm}^{3}\right)$ at a depth of $5 \mathrm{~cm}$. Source-to-chamber distance was $100 \mathrm{~cm}$, and source-to-shadow tray distance was $67 \mathrm{~cm}$. Measurements were carried out for 6 and 18 MV x-ray beams (dual energy linear accelerator ELECTA SLi Plus, University hospital of Patras, Greece). The absorbers, enough to cover the entire field, were 
placed on a tray and the beam transmission was measured while changing the thickness of absorbers. The field size was $5 \times 5 \mathrm{~cm}^{2}$ at $100 \mathrm{~cm}$ from the source for measurements with lead, brass and cerrobend, and $4 \times 4 \mathrm{~cm}$ for the polymer-metal composite materials. The transmission, $\mathrm{T}(\mathrm{x})=\mathrm{I} / \mathrm{I}_{0}$, was taken as the ratio of the detector readings with and without any material in the beam. The effective attenuation coefficients $\mu_{\text {measur }}$ were derived from x-rays attenuation curves in the materials under assumption that the attenuation curves are simply exponential (Eq. 6-1).

Table 6-3 shows the quality indices (QI) of $6 \mathrm{MV}$ and $18 \mathrm{MV}$ x-rays beams, measured with and without tray. Quality index is $\mathrm{QI}=\mathrm{TMR}_{20} / \mathrm{TMR}_{10}$, where $\mathrm{TMR}_{20}$ and $\mathrm{TMR}_{10}$ are tissue maximum ratios in water phantom at depths 20 and $10 \mathrm{~cm}$, respectively, for field size of $10 \times 10 \mathrm{~cm}^{2}$. The values of QI presented in Table 6-3 show that the introduction of the plastic tray in the beam does not change significantly the quality indices. Therefore, the measurements were carried out with the plastic tray and the attenuators were placed on the tray

TABLE 6-3. Quality indices for $6 \mathrm{MV}$ and 18 MV beams.

\begin{tabular}{ccc}
\hline \hline $\begin{array}{c}\text { Energy, } \\
\text { MV }\end{array}$ & With tray & Without tray \\
\hline 6 & 0.686 & 0.688 \\
18 & 0.778 & 0.779 \\
\hline \hline
\end{tabular}
to facilitate the procedure.

\subsubsection{Simulation studies for MCRTS verification}

The experimental setup and the properties of the attenuating materials were reproduced and computations of the doses under the different thicknesses of attenuators were performed using the Monte Carlo Radiotherapy Simulator (MCRTS). Some difficulties were encountered in modeling of the Technon/Poly material. The information about the chemical components of the PU curing agent, which constitutes $2 \%$ of the Technon/Poly, was not available and the complex [PU resin + PU curing agent] was substituted by the PU resin chemical formula. Thus, some deviations from the measured data are expected. For verification purposes only $6 \mathrm{MV}$ beam was simulated and used for a comparison of simulated and experimental data. The simulations were performed for an adequate range of thicknesses for each attenuator material in order to construct the attenuation curves based on the results of simulations and to compare them with the measured values. The transmission, $\mathrm{T}(\mathrm{x})=\mathrm{I} / \mathrm{I}_{0}$, was calculated as the ratio of the dose values with and without a given material in the beam at 5 
cm depths in the PMMA phantom. The values for the transmission curves of simulated data were derived from the PDD curves in the phantom, irradiated by the beams that were attenuated by slabs of materials of different thicknesses. Transmission curves for simulated data were plotted and the effective linear attenuation coefficients $\mu_{\text {simul }}$ were derived, assuming exponential beam attenuation (according to Eq. 6-1).

The number of photons histories was not less than $6.3 \times 10^{8}$. It was enough to keep the dose variance below one percent. The electron and photon cutoff energies were set to 0.1 and $0.01 \mathrm{MeV}$, respectively. Production of Bremsstrahlung photons and knock-on electrons were considered for energies above $0.01 \mathrm{MeV}$. The fractional energy loss step was chosen to be $4 \%$. The dose matrices voxel resolution was defined to be $5 \times 5 \times 5 \mathrm{~mm}^{3}$. The most simulations for verification studies were run on the Linux cluster of 24 PC-like microprocessors systems, called BITUNIT (http://bme.med.upatras.gr/bit) that significantly accelerated the acquisition of the 3D dose distributions (Bliznakova et al. 2006). Some of the simulations, mainly with smallest thicknesses of absorber, were run on Intel Pentium IV PC with two dual-core processors with 4Gb RAM, operating at 2,4 GHz under Windows XP. The time, necessary for receiving 3D dose matrix for a beam attenuated by an absorber, on this computer, ranged from 7 days and higher, depending on the thickness and the properties of the absorber used.

\subsubsection{Evaluation and comparison of the simulated and measured data}

First, the simulated values of the beam transmission through the studied materials were estimated. The root-mean-square error for the attenuation curve's points $\left[T_{\text {simul }}\right]_{i}$ of the simulated beam was calculated by means of comparison with the values $\left[\exp \left(-\mu_{\text {measur }} x_{i}\right)\right]$ of the exponential attenuation curve, that was fitted to the measured data, according to

$$
\mathrm{RMS}=\sqrt{\frac{1}{\mathrm{M}} \sum_{\mathrm{i}=1}^{\mathrm{M}}\left(\exp \left(-\mu_{\text {measur }} \mathrm{x}_{\mathrm{i}}\right)-\mathrm{T}_{\text {sumul }}\right)^{2}}
$$

for $\mathrm{M}$ data points.

For an estimation of a precision of the both simulated and measured effective linear attenuation coefficients that are functions of a transmitted intensity $\mathrm{T}=\mathrm{I} / \mathrm{I}_{0}$ and the absorber thickness $\mathrm{x}$, the error propagation formula was used 


$$
\Delta \mu=\sqrt{\left(\frac{\partial \mu}{\partial T}\right)^{2} \Delta T^{2}+\left(\frac{\partial \mu}{\partial x}\right)^{2} \Delta x^{2}}
$$

The final expression for error calculation is

$$
\Delta \mu=\sqrt{\left(\frac{1}{T x}\right)^{2} \Delta T^{2}+\left(\frac{\ln T}{x^{2}}\right)^{2} \Delta x^{2}},
$$

where each item is the average for all simulated or measured data points. The error of the transmittance $\Delta \mathrm{T}$ is the deviation of the measured or simulated values from the values of the exponential attenuation curve. The error of the attenuator thickness $\Delta \mathrm{x}$ includes both instrumental and random errors of thickness measurements.

The quality of the attenuators was also expressed in terms of Half-Value Layer (HVL), which is the thickness of a material required to reduce the intensity of a narrow beam to a half of its initial value. The relation between $\mu$ and HVL is as following:

$$
\mathrm{HVL}=\frac{0.683}{\mu}
$$

The relative error of HVL is equal to the relative error of the corresponding effective linear attenuation coefficient, thus absolute error of HVL is

$$
\Delta(\mathrm{HVL})=\frac{\Delta \mu}{\mu} \cdot \mathrm{HVL} .
$$

\subsubsection{Simulation studies of rotational irradiation}

3D dose distributions in homogeneous water phantoms for rotational radiotherapy with cylindrical protectors were calculated using MCRTS. The experimental setup used in simulations is shown in Fig. 6-1. The diameter of cylindrical water phantom was $15 \mathrm{~cm}$ and the length was $20 \mathrm{~cm}$. Protectors were positioned at different distances $(\mathrm{z})$ from the isocenter with diameters (d) ranged from 1.0 to $2.0 \mathrm{~cm}$. Attenuated field width $\mathrm{d}_{\mathrm{c}}$ (i.e. width of projection of the protector on the isocentric plane) varied from 1.47 to $2.94 \mathrm{~cm}$, depending on diameter and z-coordinate. The attenuation materials, used as beam absorbers, consisted of lead, tungsten, gold, Cerrobend and tungsten powder filled nylon 6. Their physical density, composition and atomic numbers are summarized in Table 6-4. 

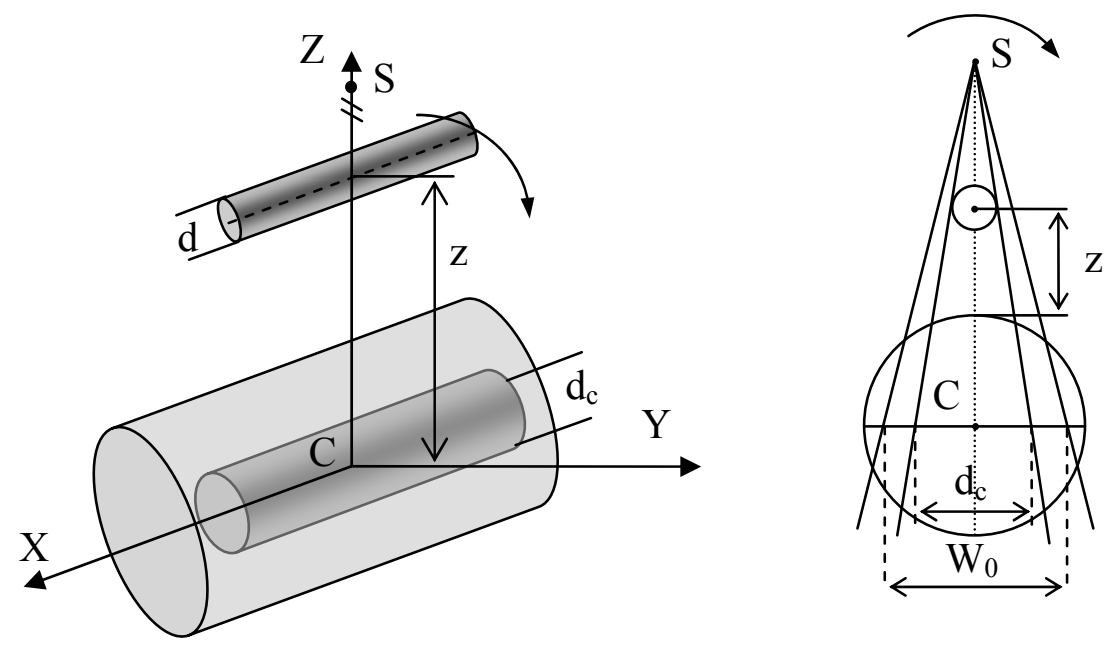

FIGURE 6-1. Schematic representation of the setup used in simulation studies. $\mathrm{W}_{0}$ is the open field size, $d_{c}$ is the field width projected by the cylindrical protector of diameter $d$ on the isocenter, $h$ is the distance between the protector and phantom surface and $\mathrm{z}$ is protector $\mathrm{z}$-coordinate. $\mathrm{S}$ is the source and $\mathrm{C}$ is the isocenter.

Dose distributions as well as dose $\left(D_{c}\right)$ at the center of the OAR were studied as a function of protector material and diameter $\mathrm{d}$, distance $\mathrm{z}$ and field size $\mathrm{W}_{0}$.

Additionally, the relative dose due to primary radiation below the protector at point $\mathrm{C}$ was calculated. The calculations were done in two ways:

(i) Monoenergetic calculations were performed using weighted mean effective linear attenuation coefficients according to exponential attenuation law, represented by Eq. 6-1. The weighted mean effective linear attenuation coefficient was determined by using weighted averaging procedure (Van Dyk 1986)

$$
\bar{\mu}=\frac{\int \mu \cdot N \cdot E d E}{\int N \cdot E d E},
$$

where $\mathrm{N}$ represents the number of photons at energy interval $\mathrm{E}+\Delta \mathrm{E}$ and integral is considered

TABLE 6-4. Materials used as beam absorbers in simulation studies with rotational geometry.

\begin{tabular}{llll}
\hline \hline Material & Density, $\mathbf{g} / \mathbf{c m}^{\mathbf{3}}$ & $\mathbf{Z}$ & Composition, (wt. \%) \\
\hline Gold & 19.32 & 79 & $\mathrm{Au}(100)$ \\
Tungsten & 19.30 & 74 & $\mathrm{~W}(100)$ \\
Lead & 11.35 & 82 & $\mathrm{~Pb}(100)$ \\
Tungsten powder filled nylon 6 & 12.00 & $72.7^{\S}$ & $\mathrm{W}: \mathrm{C}_{6} \mathrm{H}_{11} \mathrm{ON}(97: 3)$ \\
Cerrobend & 10.00 & $75.3^{\S}$ & $\mathrm{Sn}: \mathrm{Bi}: \mathrm{Pb}: \mathrm{Cd}(13.3: 50: 26.7: 10)$ \\
\hline \hline
\end{tabular}

$\S$ The effective atomic number for compounds calculated using $\mathrm{Z}^{3.5}$ dependence (Johnes and Cunningham 1983). 
over the total spectrum. The calculated values of weighted mean effective linear attenuation coefficient for gold, tungsten and lead were $0.8998 \mathrm{~cm}^{-1}, 0.8707 \mathrm{~cm}^{-1}$ and $0.5344 \mathrm{~cm}^{-1}$, correspondingly, for hardened $6 \mathrm{MV}$ spectrum of ELECTA SL75-5 linear accelerator.

(ii) In the second method, primary radiation transmission values were calculated using photon spectral distribution, i.e.,

$$
\frac{\bar{I}}{I_{0}}=\frac{\sum_{i=1}^{n} E_{i} N_{i} e^{-\mu_{i} d}}{\sum_{i=1}^{n} E_{i} N_{i}} .
$$

It was interesting also to estimate scatter contribution to the dose at the center of the OAR. The scatter from the attenuator constitutes one part of the total scatter in the phantom. Van Dyk (1986) showed by performing measurements in air that the difference between the narrow and broad beam transmissions is due to the scatter in the attenuator. The second component that contributes the total amount of the scattered radiation is in-phantom scatter. Subtracting primary dose $\mathrm{P}$ (or transmittance) from the total dose $\mathrm{D}$, calculated by Monte Carlo, we estimated scatter S contribution to the center of the OAR. Scatter-to-primary dose ratio $\mathrm{S} / \mathrm{P}$, which represents the ratio between the dose due to the scatter from the attenuator reaching a given point and the primary dose at the same point without attenuator, was also calculated.

Photons scatter from an attenuator has a relation with the mean-free path of photons in the attenuator material. Let us recall its definition. A mean-free path (or mean range) is the average distance between two successive interactions of a photon and is calculates as an inverse effective attenuation coefficient $1 / \mu$. Scatter increases with increase of material thickness, reaches the maximum at around one mean-free path and after decreases. The calculated mean-free path values for gold and tungsten are $1.11 \mathrm{~cm}\left(1 / 0.9889 \mathrm{~cm}^{-1}\right)$ and 1.15 $\mathrm{cm}\left(1 / 0.8708 \mathrm{~cm}^{-1}\right)$, respectively, if weighted mean effective linear attenuation coefficients are used. So, for studied protector diameter's range, with the smallest diameter of $1 \mathrm{~cm}$, it is expected that the scatter will decrease with increase in diameter for gold and tungsten. For lead mean-free path is $1.87 \mathrm{~cm}\left(1 / 0.5344 \mathrm{~cm}^{-1}\right)$, so scatter contribution for the studied diameters range (from 1.0 to $2.0 \mathrm{~cm}$ ) is expected first to increase.

To investigate the influence of the beam hardening effect, contaminant electrons and scattered photons on the surface dose in the presence of different cylindrical attenuators, the dose to a $2 \mathrm{~mm}$ water layer, i.e., in the first voxel layer, under the phantom surface along its 
length was calculated, analyzed and compared for different experiments. The average electron ranges for materials used as beam absorbers were calculated in order to assess the possible effect of the material thickness to the beam contamination with the secondary electrons. The continuous-slowing-down approximation (CSDA) range, which is a very close approximation to the average path length traveled by a charged particle as it slows down to rest, was used instead for this purpose. The data for lead, gold and tungsten were taken from ESTAR: Stopping Powers and Ranges for Electrons database (Berger et al. 2005).

The Klein-Nishina equation describes the relative probability for a photon to scatter through an angle $\theta$ (see Eq. 6-3). For all megavoltage beam energies the relative probability is highest for small scatter angles. At $6 \mathrm{MV}$ with effective beam energies of $2 \mathrm{MeV}$ this photon scatter angle will be within $45^{\circ}$ (du Plessis and Willemse 2005). At these scatter angles and effective photon beam energies the electrons will have maximum energies of $1.1-1.4 \mathrm{MeV}$ for $6 \mathrm{MV}$ and as can be calculated from $h v^{\prime}=h v /[1+\alpha(1-\cos \theta)]$, where and $\alpha=h v / 0.511, h v$ is the original energy of the photon, and $h v^{\prime}$ is the energy of the scattered photon, with the electron energy, given by $E=h v-h v$ '. The CSDA ranges for the electrons based on the above energy of the electrons produced in attenuators are $0.6 \mathrm{~mm}$ for gold and tungsten and $1 \mathrm{~mm}$ for lead. From these values follows that most of the electrons must be produced within the bottom $1 \mathrm{~mm}$ layer to exit the protector as further upstream produced electrons are absorbed in the protector.

Scatter from collimator was not simulated, thus the existing surface dose is mostly due to beam contamination by the electrons produced in the protector and backscattered radiation. The surface dose values were normalized to the dose at the isocenter of the open beam. The number of photons scoring to the surface dose is small that results in large statistical fluctuations. Thus, to decrease statistical fluctuations, the surface dose along four diametrically opposite surface layers was added. Additionally, the mean smoothing filter with kernel size 3 was applied, thus replacing each voxel value of the profile with the mean value of its neighbors, including itself.

The dose gradient just outside the shielded region was estimated in the work. The dose gradient $\Delta \mathrm{D} / \Delta \mathrm{r}$ was calculated as a difference of the dose values in adjacent voxels along the radius of the phantom. The voxel dimensions were $2 \times 2 \times 2 \mathrm{~cm}^{2}$, thus the gradient dimensions were $\% /$ voxel length or $\% / 2 \mathrm{~mm}$. The dose augmentation at the border of a compensated field, where we are interested in the gradient value, is not linear, so, it was decided to leave gradient's dimensions as \%/voxel length and not to divide its value by two for calculation of a 
dose change per $1 \mathrm{~mm}$. The dose gradient function is not smooth, as MC simulation data have statistical fluctuations. However, the maximal gradient value can be distinguished clearly and its spatial position can be found with accuracy. The location of the maximal gradient has been studied for different attenuator materials, location and dimensions as well as its numerical value.

Dose distributions were obtained using photon fan beams, originated from a source of size $1 \times 1 \mathrm{~mm}^{2}$ with a source-to surface distance (SID) of $100 \mathrm{~cm}$ for three field sizes $\mathrm{W}_{0}(10$ $\times 10,7.5 \times 7.5$ and $5 \times 5 \mathrm{~cm}^{2}$ at SID). A $6 \mathrm{MV}$ x-ray beam (ELECTA SL75-5, University hospital of Patras) with hardened energy spectrum (see Chapter 5, section 5.2.3) was used in simulations. The gantry rotation performed in simulations was from $0^{\circ}$ to $360^{\circ}$ with discrete step of $2^{\circ}$. For the MC simulations, the electron and photon cutoff energies were set to 0.1 and $0.01 \mathrm{MeV}$, respectively. Production of Bremsstrahlung photons and knock of electrons were considered for energies above $0.05 \mathrm{MeV}$. The fractional energy loss step was chosen to be $10 \%$. The dose matrices were defined with $2 \times 2 \times 2 \mathrm{~mm}^{3}$ voxel resolution.

\subsection{RESULTS}

\subsubsection{Verification studies}

A comparison of measured and simulated percent attenuation curves of the tested materials is presented in Fig. 6-2. The values of the relative dose at a depth of $5 \mathrm{~cm}$ (normalized to the open beam dose at this depth) were used for attenuation curves plot. Figure 6-2(a) shows the percent transmission curves for lead, cerrobend and brass for a narrow beam geometry $\left(5 \times 5 \mathrm{~cm}^{2}\right.$ field size $)$ as a function of an absorber thickness. Figure 6-2(b) shows the percent attenuation curves for the metal-polymer composite materials for $4 \times 4$ field size. The exponential curves, fitted to the measured data, are also shown. Figure 6-3 illustrates the family of PDD curves in lead, normalized to the value of the open beam dose at a depth of 5 $\mathrm{mm}$, from which the values for the attenuation curves of simulated data were derived.

The values of the effective linear attenuation coefficient $\mu_{\text {simul }}$ and $\mu_{\text {measur }}$ and HVL derived from simulated and measured attenuated curves, as well as the results of the statistical analysis of the simulated data are summarized in Table 6-5. The effective linear attenuation coefficients were found from fitting the experimental and simulated data points by exponential curves. The R-squared, which is a statistical measure of how well a regression 
(a)

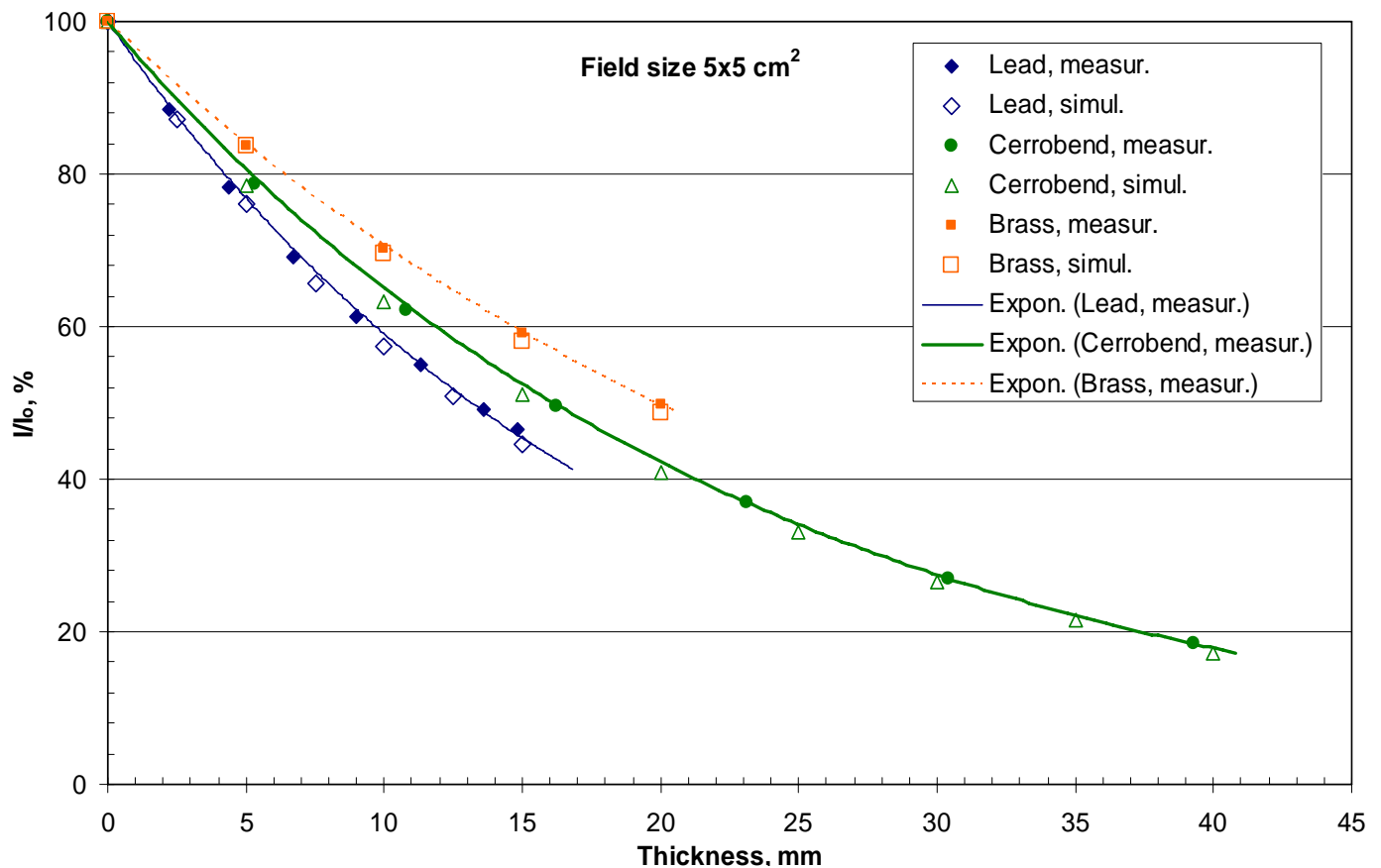

(b)

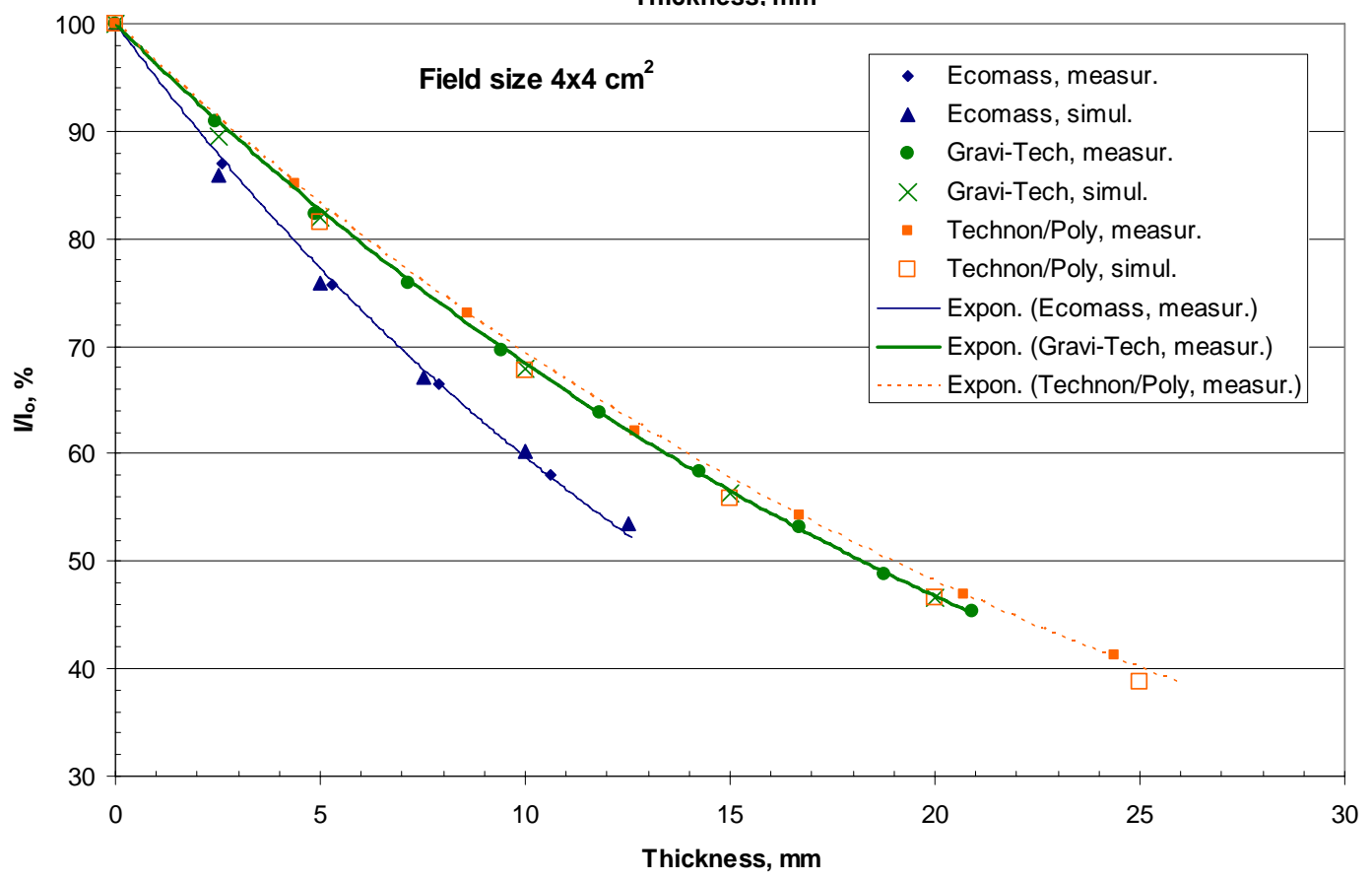

FIGURE 6-2. Comparison of the simulated and measured transmission data of primary protons of energy of 6MV through (a) lead, cerrobend and brass and (b) metal- polymer composites. The properties of the materials are presented in Table 6-2.

line approximates real data points, for all data fitted to the exponential curves were not smaller than 0.995 (with 1 corresponding to the ideal coincidence). The RMS error calculated using Eq. 6-6 and included in the table shows the deviation of the simulated data points from the exponential curve fitted to the measured data. 


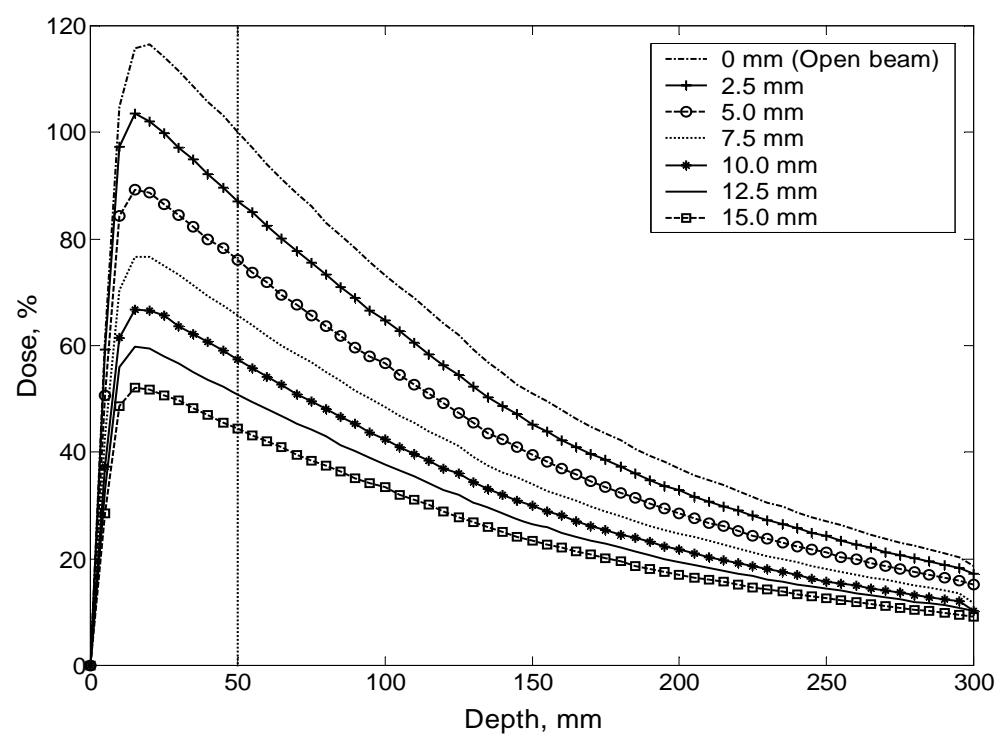

FIGURE 6-3. The family of PDD curves in a PMMA phantom for a stationary beam attenuated by the lead slabs of different thicknesses.

TABLE 6-5. Effective linear attenuation coefficients and HVLs, derived from the measured and simulated attenuation data in different materials for $6 \mathrm{MV}$ beam. RMS error shows the discrepancy between the simulated data points and the exponential attenuation curves, fitted to the measured data. Absolute and relative error for all values of effective linear attenuation coefficients and HVLs, as well as discrepancies between the "measured" and "simulated" effective linear attenuation coefficients are shown.

\begin{tabular}{|c|c|c|c|c|c|c|c|}
\hline & \multirow{2}{*}{ Material } & \multirow{2}{*}{$\begin{array}{c}\text { RMS } \\
\text { error, } \\
\%\end{array}$} & \multicolumn{2}{|c|}{ Measured } & \multicolumn{2}{|c|}{ Simulated } & \multirow{2}{*}{$\begin{array}{c}\frac{\mu_{1}-\mu_{2}}{\mu_{1}} \\
\mathbf{\%}\end{array}$} \\
\hline & & & $\mu_{1}, \mathbf{c m}^{-1}$ & HVL, cm & $\mu_{2}, \mathbf{c m}^{-1}$ & HVL, cm & \\
\hline 1 & Lead & 1.1 & $\begin{array}{r}0.527 \pm 0.026 \\
4.8 \%\end{array}$ & $1.31 \pm 0.06$ & $\begin{array}{r}0.545 \pm 0.007 \\
1.3 \%\end{array}$ & $1.27 \pm 0.02$ & 3.4 \\
\hline 2 & Cerrobend & 1.3 & $\begin{array}{r}0.431 \pm 0.009 \\
2.0 \%\end{array}$ & $1.61 \pm 0.03$ & $\begin{array}{r}0.442 \pm 0.009 \\
2.1 \%\end{array}$ & $1.57 \pm 0.03$ & 2.6 \\
\hline 3 & Brass & 1.0 & $\begin{array}{r}0.349 \pm 0.008 \\
2.3 \%\end{array}$ & $1.99 \pm 0.05$ & $\begin{array}{r}0.361 \pm 0.003 \\
0.7 \%\end{array}$ & $1.92 \pm 0.01$ & 3.4 \\
\hline 4 & Ecomass & 1.2 & $\begin{array}{r}0.516 \pm 0.022 \\
4.3 \%\end{array}$ & $1.34 \pm 0.06$ & $\begin{array}{r}0.513 \pm 0.034 \\
6.6 \%\end{array}$ & $1.35 \pm 0.09$ & 0.6 \\
\hline 5 & Gravi-Tech & 0.8 & $\begin{array}{r}0.380 \pm 0.011 \\
3.0 \%\end{array}$ & $1.82 \pm 0.05$ & $\begin{array}{r}0.384 \pm 0.016 \\
4.1 \%\end{array}$ & $1.80 \pm 0.07$ & 1.1 \\
\hline 6 & Technon/Poly & 2.1 & $\begin{array}{r}0.365 \pm 0.007 \\
2.0 \%\end{array}$ & $1.90 \pm 0.04$ & $\begin{array}{r}0.382 \pm 0.013 \\
3.5 \%\end{array}$ & $1.81 \pm 0.06$ & 4.7 \\
\hline
\end{tabular}




\subsubsection{Measurements with $18 \mathrm{MV}$ beam attenuation}

The experimental measurements of the attenuation of the $18 \mathrm{MV}$ beam by the same materials and the best fit exponential curves are presented in Fig. 6-4. The linear attenuation coefficient is a function of beam energy as the photon interaction process changes with energy. The $18 \mathrm{MV}$ beam is slightly more penetrating. This shows also Table $6-6$, where the

(a)

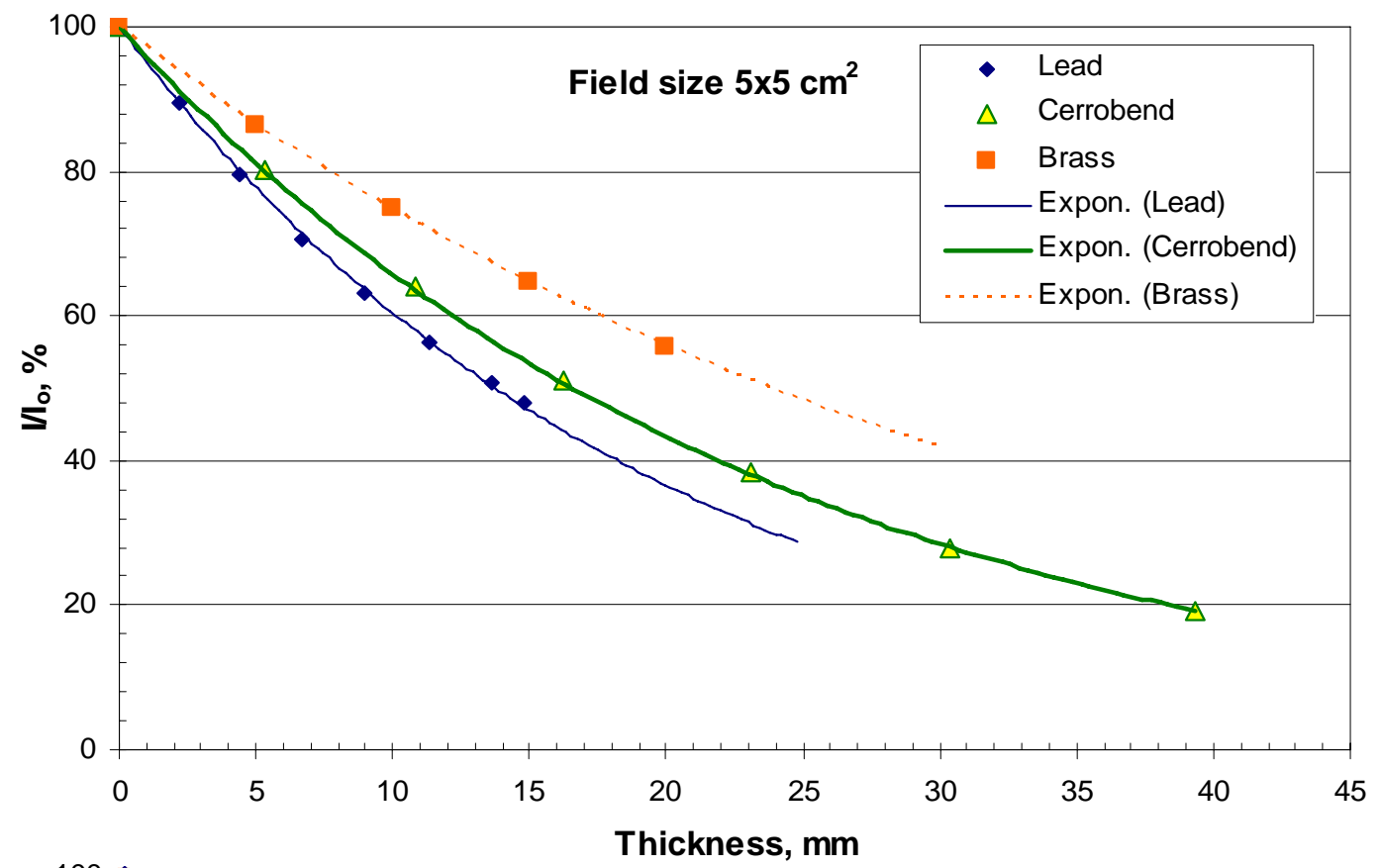

(b)

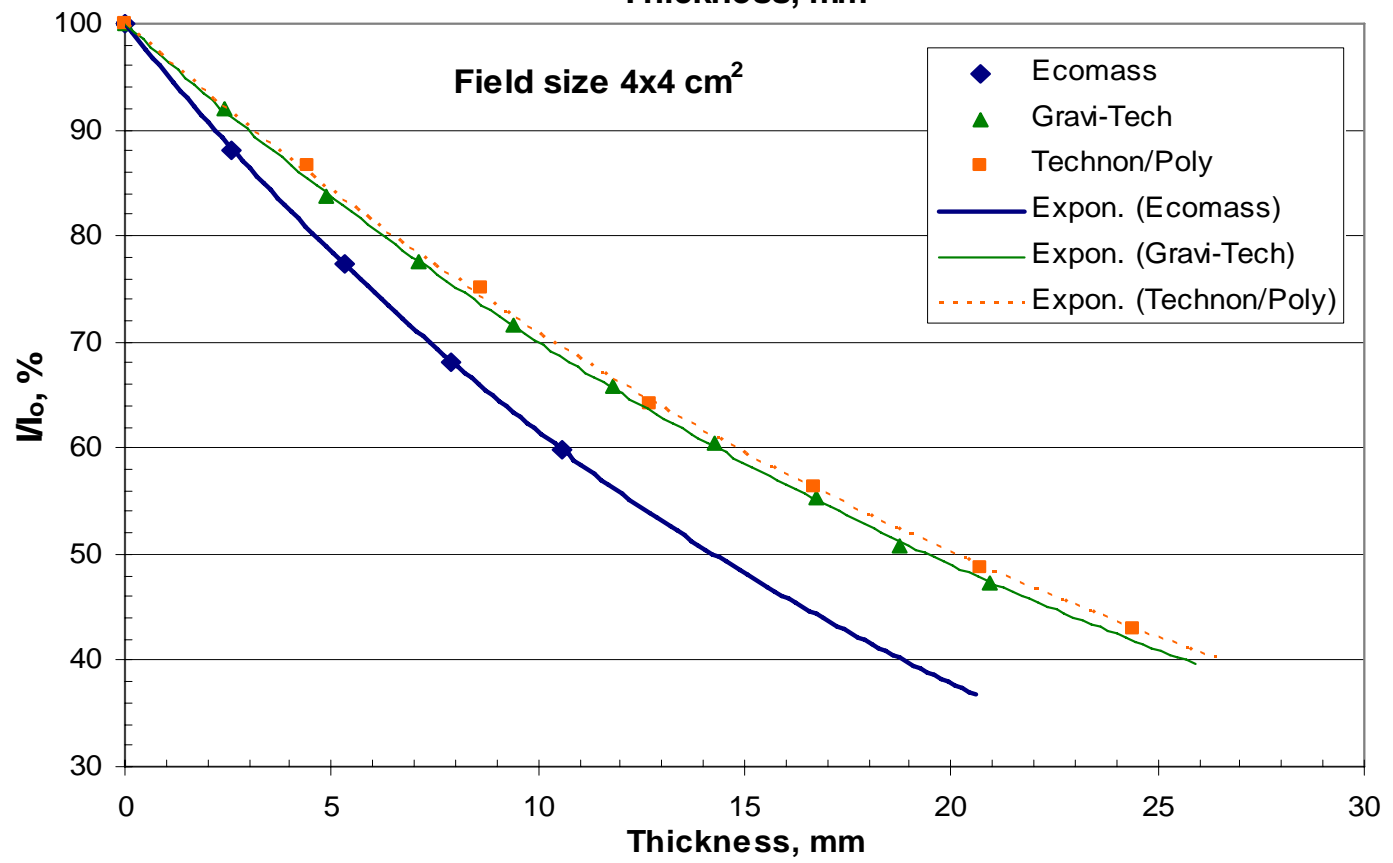

FIGURE 6-4. Measured percent transmission curves for narrow beam geometries of 18MV beam for (a) lead, cerrobend and brass and (b) metal-polymer composite materials. The properties of the materials are presented in Table 6-2. 
TABLE 6-6. Effective linear attenuation coefficients and HVLs derived from measured data for $18 \mathrm{MV}$ beam.

\begin{tabular}{llllll}
\hline \hline & Material & $\boldsymbol{\mu}_{\mathrm{eff}}, \mathbf{c m}^{-1}$ & $\mathbf{H V L}, \mathbf{c m}$ & $\mathbf{R}^{\mathbf{2}}$ \\
\hline 1 & Lead & $0.504 \pm 0.019$, & $3.8 \%$ & $1.38 \pm 0.05$ & 0.9993 \\
2 & Cerrobend & $0.419 \pm 0.006$, & $2.1 \%$ & $1.65 \pm 0.03$ & 0.9999 \\
3 & Brass & $0.291 \pm 0.007, \quad 1.7 \%$ & $2.38 \pm 0.05$ & 0.9999 \\
4 & Ecomass & $0.486 \pm 0.020, \quad 4.0 \%$ & $1.43 \pm 0.06$ & 0.9999 \\
5 & Gravi-Tech & $0.357 \pm 0.010,2.9 \%$ & $1.94 \pm 0.06$ & 0.9998 \\
6 & Technon/Poly & $0.345 \pm 0.008,2.5 \%$ & $2.01 \pm 0.05$ & 0.9997 \\
\hline \hline
\end{tabular}

effective linear attenuation coefficients and HVLs are presented. The drop in effective linear attenuation coefficient values as beam energy was increased from $6 \mathrm{MV}$ to $18 \mathrm{MV}$ is the result of the inverse energy dependence of the photoelectric effect and Compton scattering interactions.

\subsubsection{Simulation studies with cylindrical protectors}

Dose distributions and dose at the center of the OAR. A bulk of simulations was run to study the effect of the cylindrical protector on the dose distribution in the rotational irradiation. Figures 6-5 to 6-8 represent dose profiles along the phantom diameter for field size $10 \times 10 \mathrm{~cm}^{2}$ for different protector materials and diameters $\mathrm{d}$, distances $\mathrm{z}$ and therefore, different attenuated field widths $d_{c}$, as well. Dose values were normalized to the dose of the open beam at the isocenter. The insets reproduce configurations of protectors for the presented dose distributions. Figure 6-5 shows dose distributions for gold protectors placed at $\mathrm{z}=32 \mathrm{~cm}$, while protectors diameters $\mathrm{d}$ varied. Dose profiles for gold protectors of the same diameter $\mathrm{d}=1.46 \mathrm{~cm}$, placed at different distances $\mathrm{z}$ from the isocenter are shown in Fig. 6-6. Figure 6-7 illustrates dose profiles of protectors made of different materials with the same diameters $\mathrm{d}=1.60 \mathrm{~cm}$ and located at the same distance $\mathrm{z}=32 \mathrm{~cm}$ from the isocenter. Finally, in Fig. 6-8 dose distributions for gold protectors and the same attenuated field width at the isocenter $\mathrm{d}_{\mathrm{c}}=2.35 \mathrm{~cm}$, but for different $\mathrm{z}$ (and therefore $\mathrm{d}$ ) are plotted. 


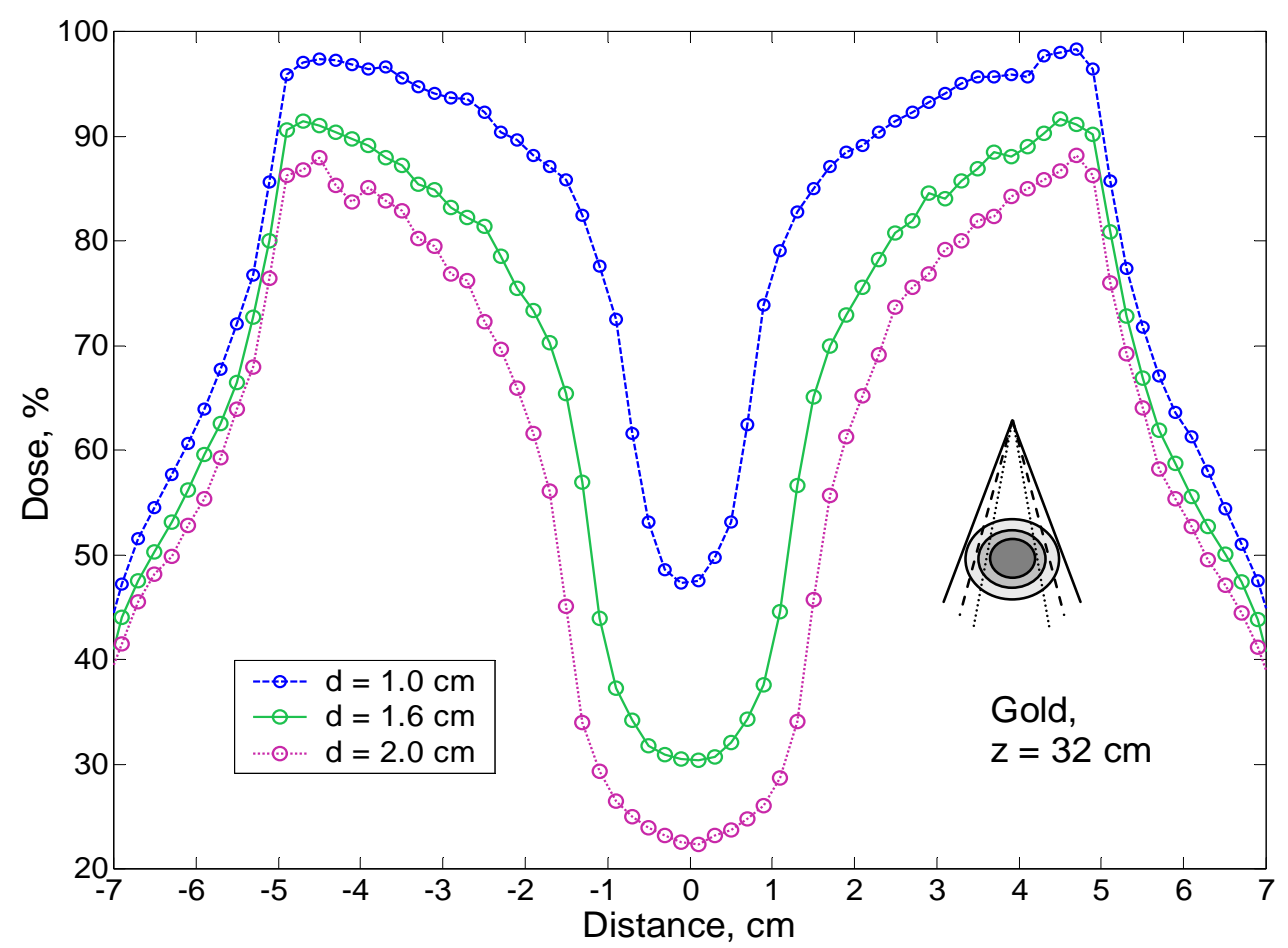

FIGURE 6-5. Transverse dose profiles shown at the center of the cylindrical phantom for $6 \mathrm{MV}$ beam for gold protector placed at constant $\mathrm{z}$ distance from the isocenter, while protector's diameter d varied.

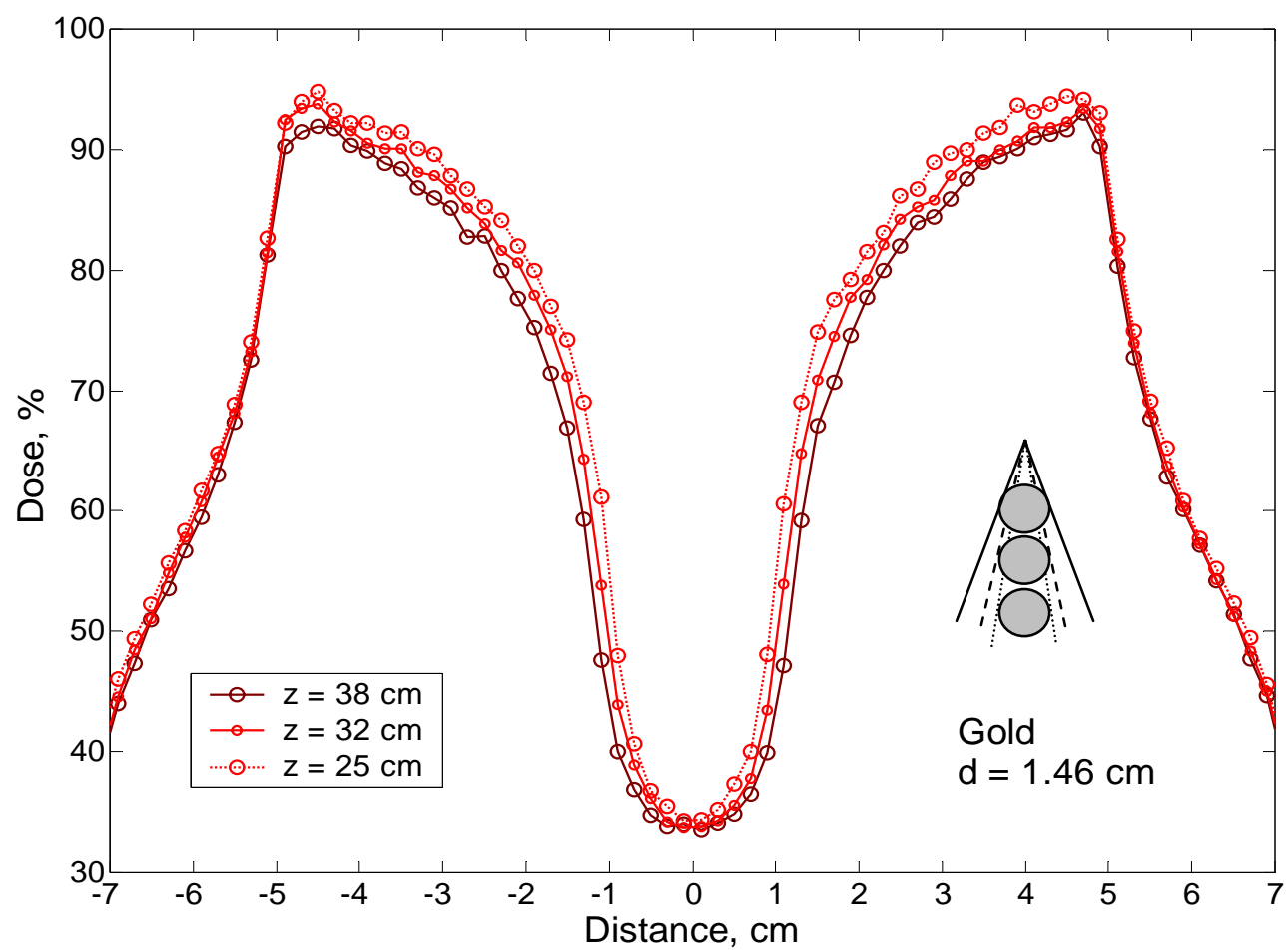

FIGURE 6-6. Transverse dose profiles at the center of the cylindrical phantom for $6 \mathrm{MV}$ beam for gold protector of constant diameter $\mathrm{d}=1.46 \mathrm{~cm}$, while their $\mathrm{z}$-coordinate varies. 


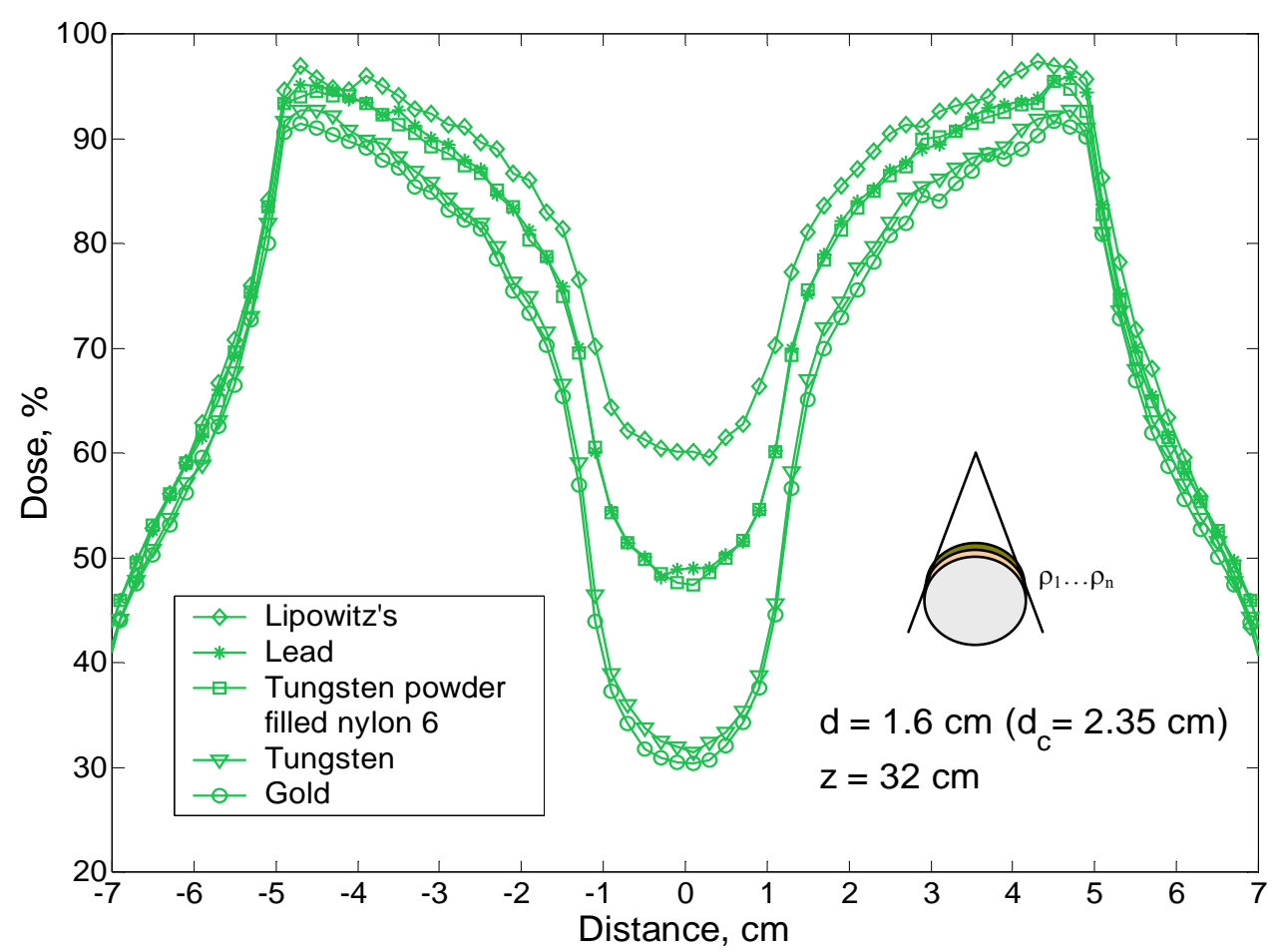

FIGURE 6-7. Transverse dose profiles at the center of the cylindrical phantom for 6 MV beam for protectors of the same diameter and placed at the same distance from the isocenter, but made of different materials.

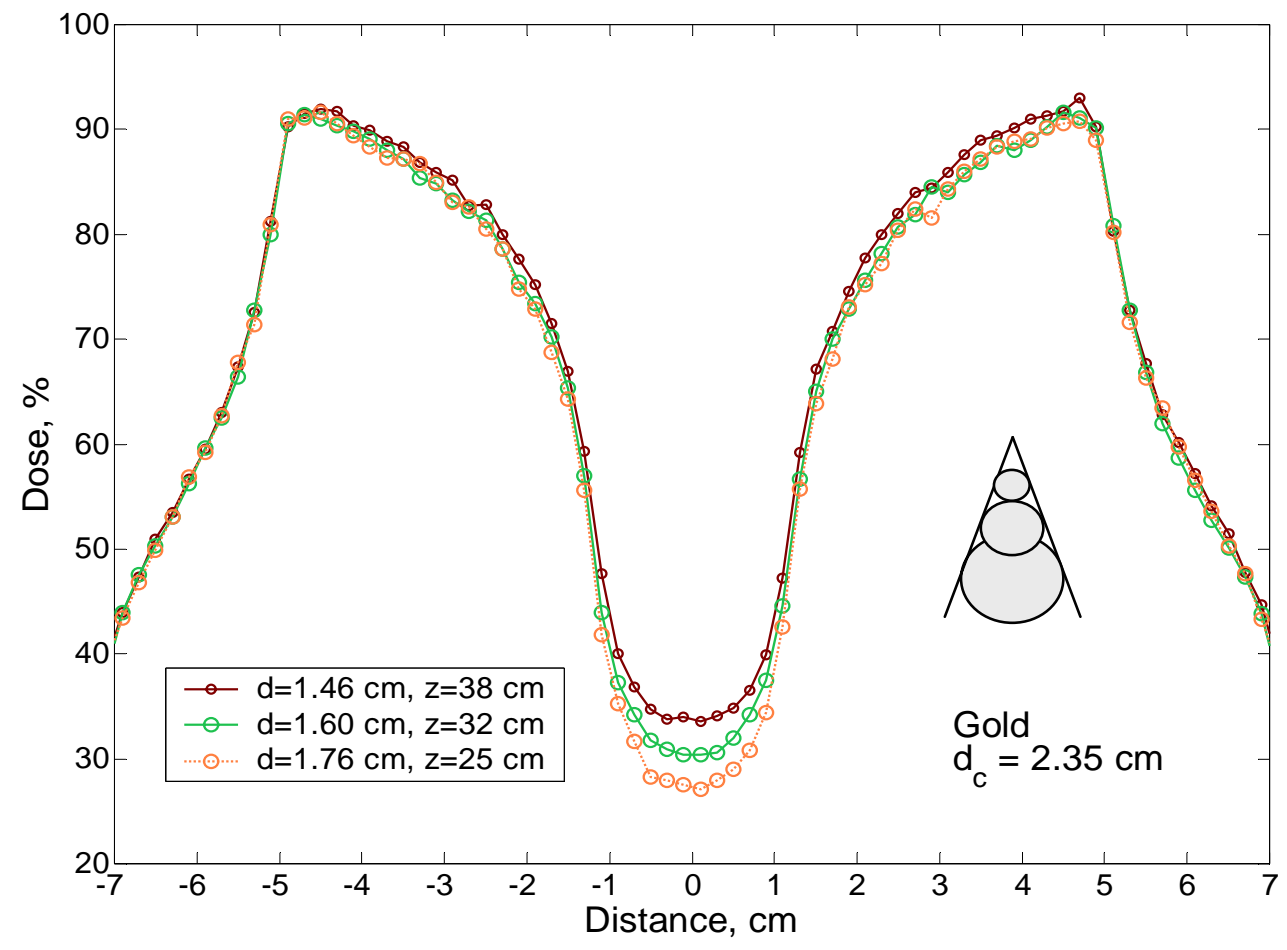

FIGURE 6-8. Transverse dose profiles shown at the center of the cylindrical phantom for $6 \mathrm{MV}$ beam for gold protectors that yield the same attenuated beam width at the isocenter. The protector's diameter varies accordingly to the $\mathrm{z}$-coordinate of the protector. 
The effect of the field size on the dose distributions in a homogeneous phantom is illustrated in Fig. 6-9. Dose distributions for tungsten protector of $1.0 \mathrm{~cm}$ and $1.6 \mathrm{~cm}$ in a diameter, placed at $\mathrm{z}=32 \mathrm{~cm}$ from the isocenter, for three different field sizes are shown. A noticeable difference in the doses at the center of the protected region is observed for both protectors' diameters.
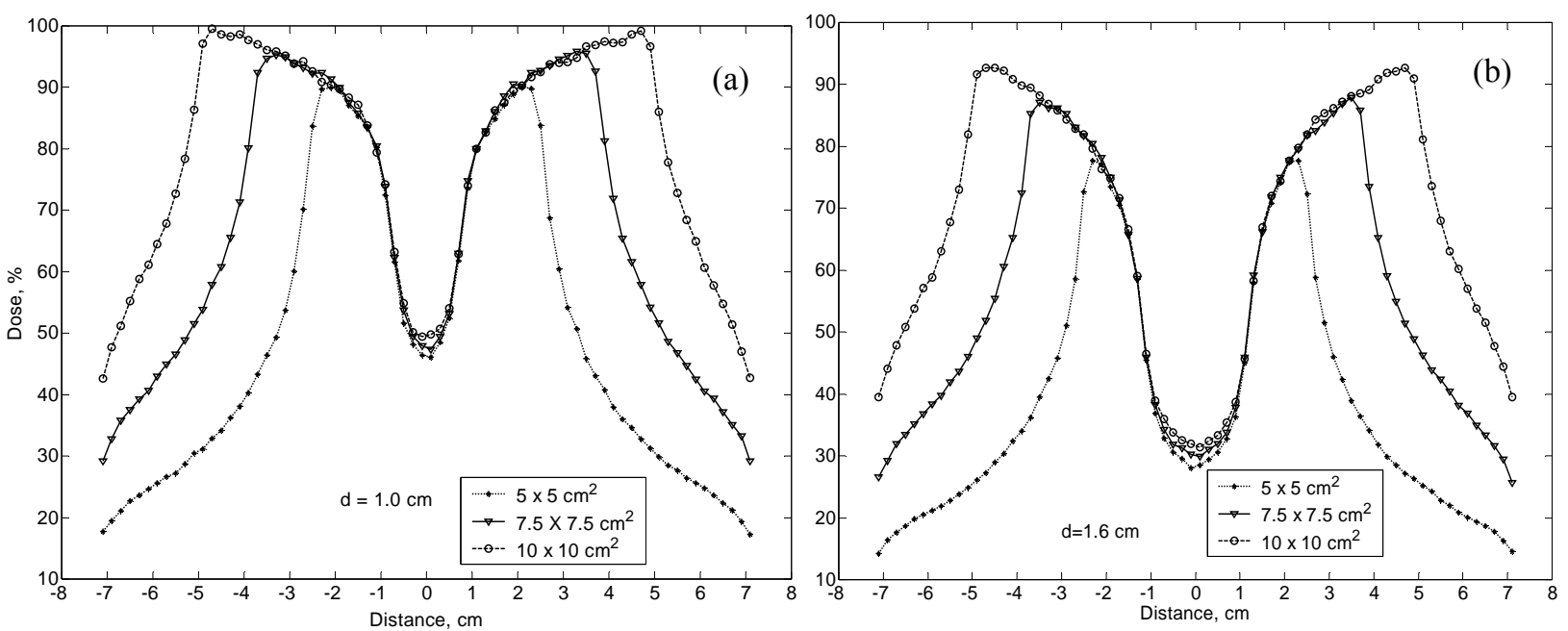

FIGURE 6-9. Transverse dose profiles at the isocenter for beams modulated with tungsten protector of (a) $1.0 \mathrm{~cm}$ and (b) $1.6 \mathrm{~cm}$ in diameter for $5 \times 5,7.5 \times 7.5$ and $10 \times 10 \mathrm{~cm}^{2}$ field sizes.

Figure 6-10 shows the dose at the center of the OAR dependence on the field size for lead, gold and tungsten protectors of 1.0 and $1.6 \mathrm{~cm}$ in a diameter. The data were parameterized and the linear regression lines are also shown in the figure. The coefficients of the straight-line equations in form of

$$
\mathrm{D}_{\mathrm{c}}=\beta \cdot \mathrm{W}_{0}+\alpha
$$

are summarized in Table 6-7, where $\mathrm{D}_{\mathrm{c}}$ is the dose at the center of the OAR, $\beta$ is the slope of the line, $\mathrm{W}_{0}$ is the side of the square field and $\alpha$ is the $D_{c}$ axis intercept (i.e. the dose at the center of the OAR for a zero field size). Due to increase in scatter contribution, the dose value increases with increasing field size for all protector's thicknesses. Zero field size dose values were obtained by extrapolating the data with linear regression analysis (they correspond to the coefficient $\alpha$ of Eq. 6-12)
TABLE 6-7. The coefficients of straight line equation in form $\mathrm{D}_{\mathrm{c}}=\beta \cdot \mathrm{W}_{0}+\alpha$ are presented. The values given in parenthesis are percent transmissions of primary beam, calculated using photons spectral distribution (Eq. 6-7).

\begin{tabular}{|c|c|c|c|}
\hline Material & $\mathrm{d}, \mathrm{cm}$ & $\alpha, \%$ & $\beta$ \\
\hline Gold & & $42.4(41.9)$ & 0.53 \\
\hline Tungsten & 1.0 & $43.3(43.1)$ & 0.60 \\
\hline Lead & & $60.0(59.6)$ & 0.35 \\
\hline Gold & & $24.7(25.1)$ & 0.58 \\
\hline Tungsten & 1.6 & $25.1(26.2)$ & 0.64 \\
\hline Lead & & $43.1(43.8)$ & 0.47 \\
\hline
\end{tabular}




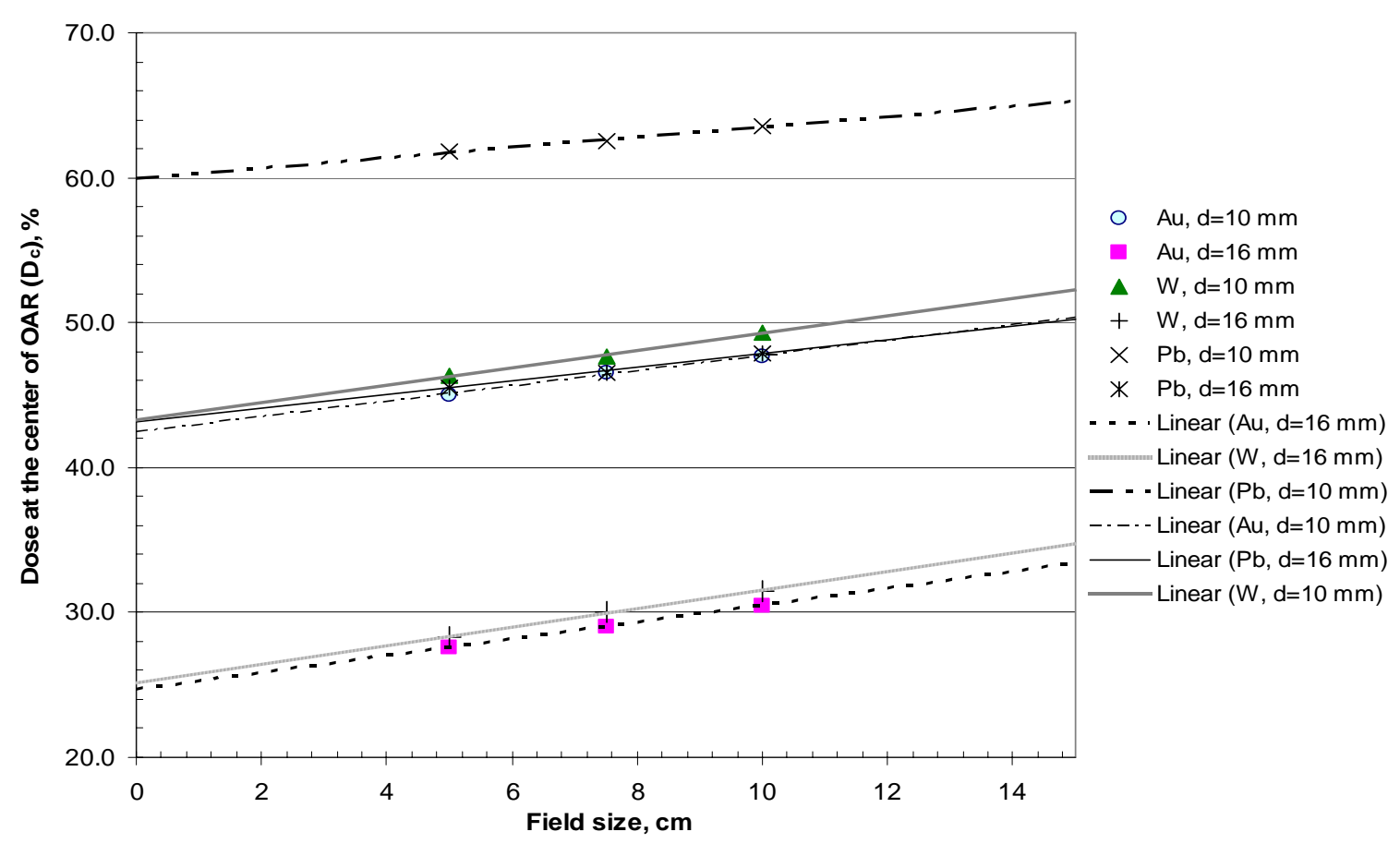

FIGURE 6-10. Dose at the center of the OAR as a function of the field size.

and are presented in Table 6-7. Additionally, the narrow beam doses at the center of the attenuated field were calculated by the exponential attenuation law, assuming that dose is proportional to energy fluence (intensity). The values have been included in Table 6-7 in parentheses.

Relative dose at the center of the OAR under attenuator $D_{c}$ for the field size $10 \times 10 \mathrm{~cm}^{2}$ as a function of the protector diameter $\mathrm{d}$ for various materials and protector's z-coordinate is shown in Fig. 6-11. Moreover, the relative dose due to primary radiation versus the protector's diameter of monoenergetic calculations with weighted mean mass attenuation coefficients (calculated using Eq. 6-10) is shown by dashed curves. Solid curves show $D_{c}$, calculated using photon spectral distribution, accordingly to Eq. 6-11. Designation of data points, resulted from MC calculations for different protector's z-coordinates, are shown in Fig. 6-11 legend. Curves for $\mathrm{z}=32 \mathrm{~cm}$, shown as dotted, were received by polynomial fit. Data points for $\mathrm{z}=25 \mathrm{~cm}$ are above the fitted curve for $\mathrm{z}=32 \mathrm{~cm}$, while for $\mathrm{z}=38 \mathrm{~cm}$ below the curve. All curves for tungsten protectors lie above the corresponding curves for gold protector.

Scatter contribution to the total (transmitted) dose as well as scatter-to-primary dose ratio at the center of the OAR as a function of the protector's diameter for $10 \times 10 \mathrm{~cm}^{2}$ field size are shown in Fig. 6-12. The scatter of lead protector increases with the diameter, while the scatter for gold and tungsten protectors shows opposite behavior. The maximum 


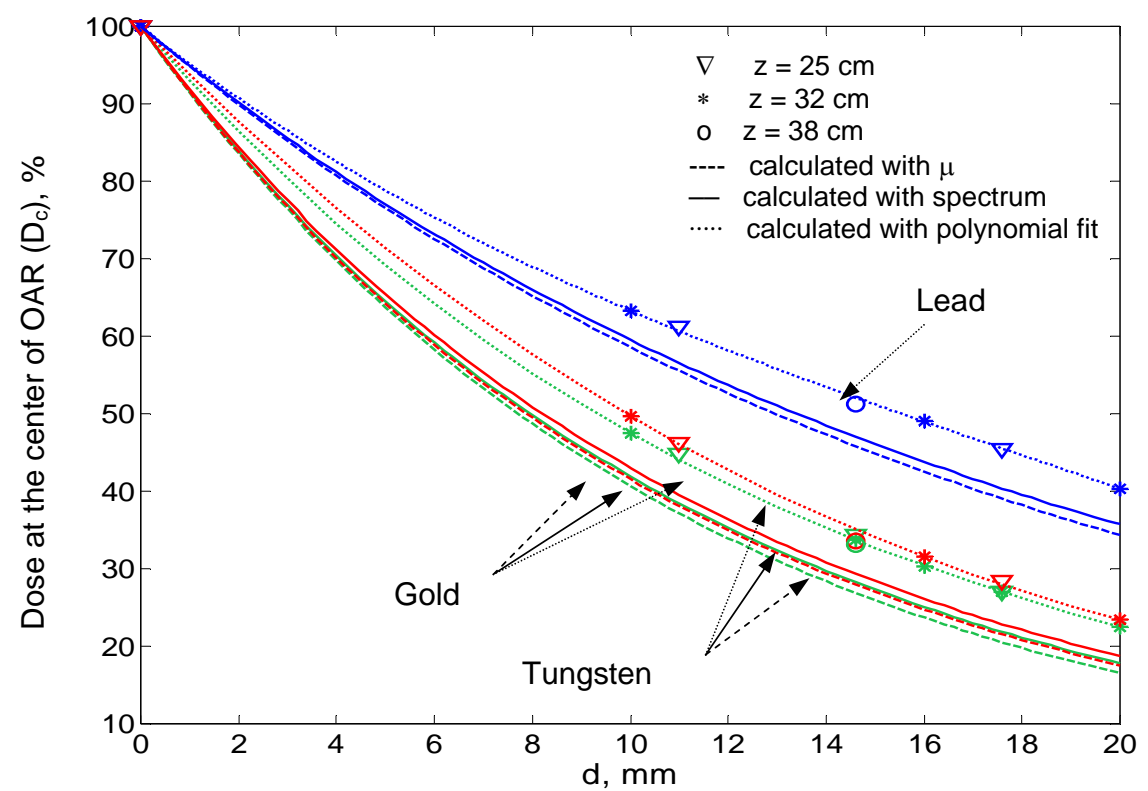

FIGURE 6-11. Dose at the center of the OAR versus protector diameter, determined by MC (data points are for $\mathrm{z}=25,32$ and $38 \mathrm{~cm}$; for $\mathrm{z}=32 \mathrm{~cm}$ dotted curve is received by polynomial fit) and analytically calculated, using weighted mean effective linear attenuation coefficient (dashed curve) and spectral distribution (solid curve).

contribution was expected for a protector's diameter corresponding to approximately one mean free path, that are around $1.1 \mathrm{~cm}$ for gold and tungsten and 1.9 for lead. Unfortunately, the dose data for these diameters were not available and this hypothesis can not be proved here. The scatter-to-primary dose ratios for all materials increase as the protector's diameter increases. So, for all materials it can be explained by the fact that the primary dose reduction due to radiation absorption in the protector is more pronounced than the change (lessening for gold and tungsten and increase for lead) in scatter contribution.
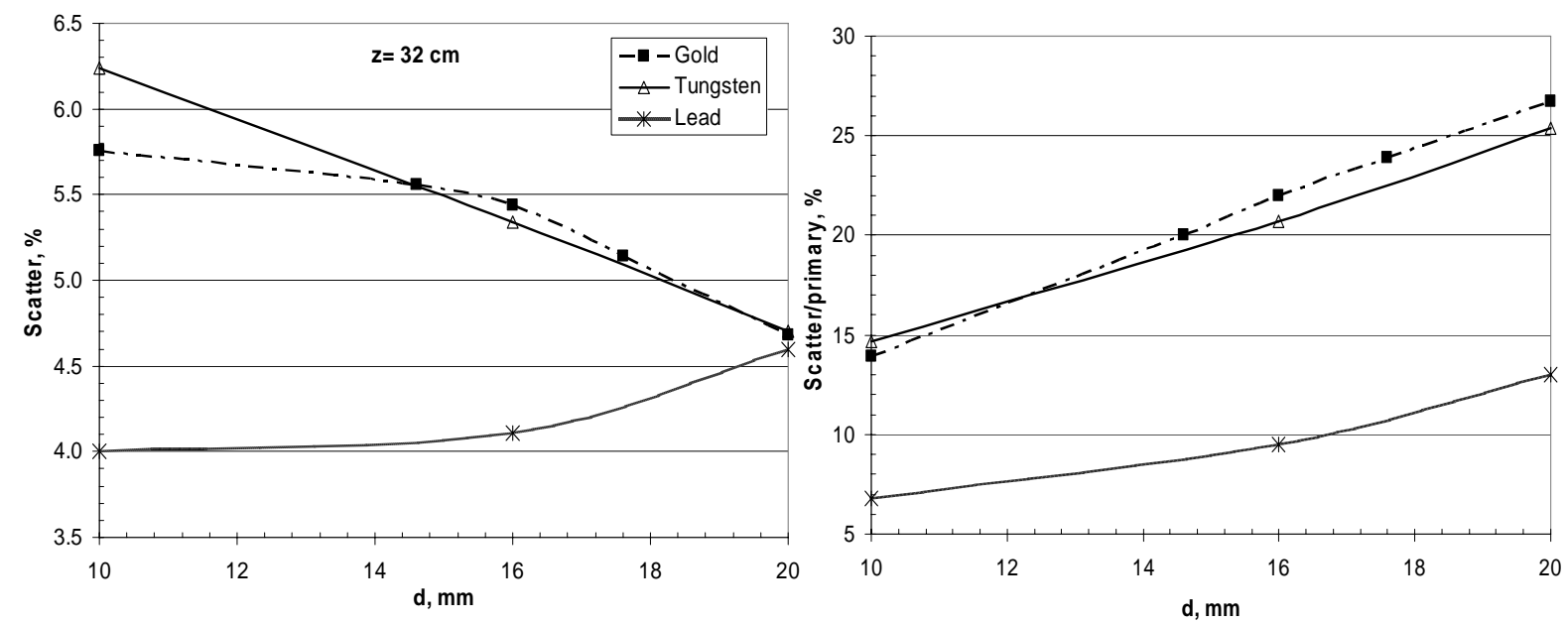

FIGURE 6-12. Scatter, contributing the total dose at the center of the OAR (a) and scatter-to-primary dose ratio versus protector's diameter. 
Figure 6-13 illustrates that scatter at the center of the OAR and scatter-to-primary dose ratio at the same points increase as the field size increases. The larger protector's diameter yield the lower scatter contribution at the center of the OAR as the scattered photons are absorbed prior to reach the center of the attenuated field. For smaller field sizes this feature is more enhanced as scatter contribution is low. The results also indicate that in-phantom scatter plays the dominant role in total scatter increase with the field size.
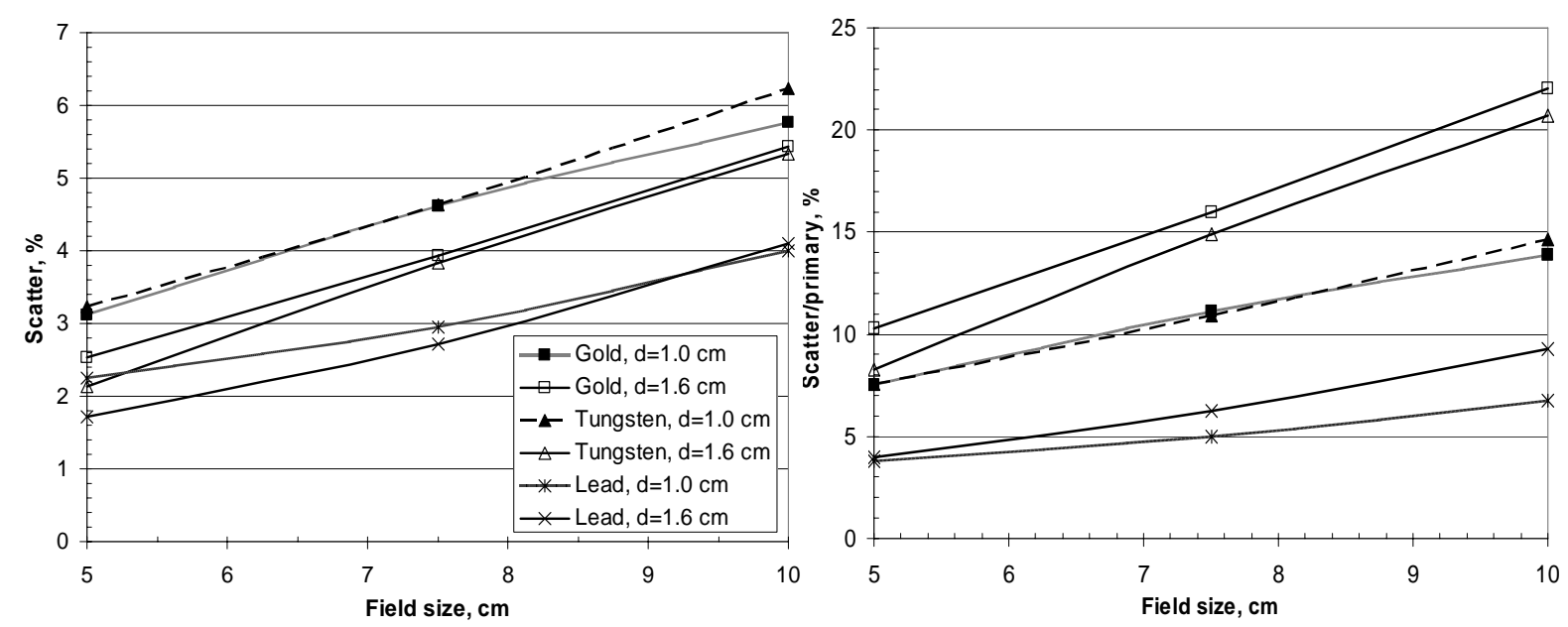

FIGURE 6-13. Scatter contributing the total dose at the center of the OAR (a) and scatter-toprimary dose ratio versus field size.

Surface dose. A surface dose along the length of phantom is shown in Figs. 6-14(a) to 6-14(e). Scatter from collimator was not modeled, thus the "additional" dose to the surface was mostly due to scattered radiation, originated from a protector and backscattered photons and electrons. The surface dose for a field size $10 \times 10 \mathrm{~cm}^{2}$ for all studied cases did not exceed $9.5 \%$ of the dose at the isocenter of the open beam. It was in the range of 7 to $9.5 \%$. The results indicate that the surface dose contribution from scattered radiation is greater for shorter protector-surface distance, as it can be seen in Fig. 6-14(a). For gold protector of diameter of $1.46 \mathrm{~cm}$ placed at $38 \mathrm{~cm}$ from the isocenter (or $31.5 \mathrm{~cm}$ from the phantom surface), the surface dose does not differ much from the phantom surface dose of open beam (see Fig. 6-14(a)). It does not mean that the protector does not contribute additional electron fluence. However, due to the beam hardening effect that takes place due to presence of an absorber, more energetic photons that pass the attenuator deposit their energy further from the surface, thus compensating the surface dose increase due to secondary electrons. When a lead protector is used, the surface dose is generally higher than for protectors made of gold or tungsten (see Fig. 6-14(b)). The absolute difference of lead with gold and tungsten protectors' 

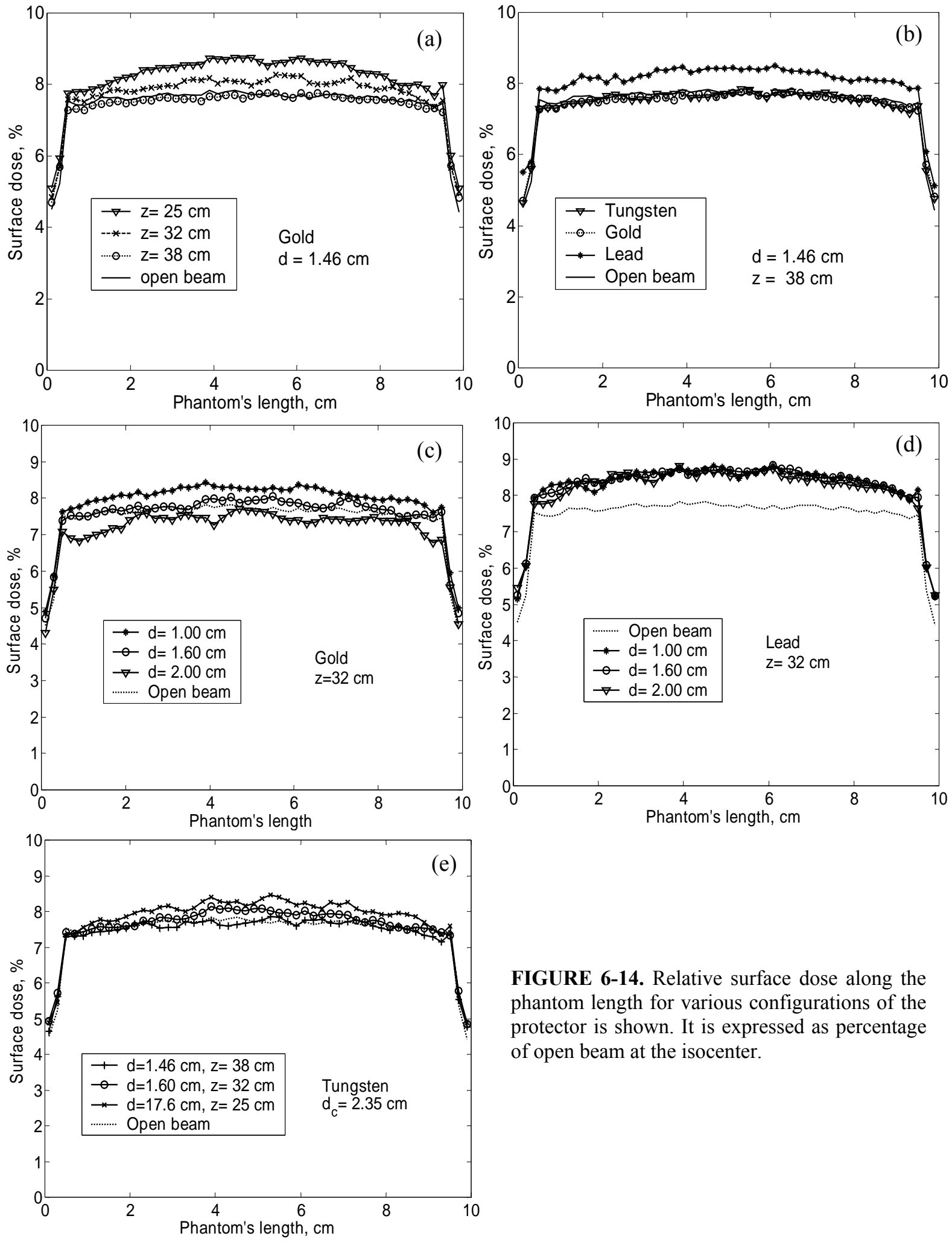

FIGURE 6-14. Relative surface dose along the phantom length for various configurations of the protector is shown. It is expressed as percentage of open beam at the isocenter.

surface doses for protectors of $1.46 \mathrm{~cm}$ in diameter and placed at $38 \mathrm{~cm}$ from the isocenter is about $0.6 \%$ (of the dose at the isocenter of the open beam). The surface dose due to gold and tungsten protectors of the same parameters used in rotational irradiation is nearly the same by magnitude. Increase in atomic number $\mathrm{Z}$ results in increased surface dose due to increased 
production in of photoelectrons and electron pair in addition to Compton electrons (Khan 1994). Another reason of an increased lead surface dose is that the average electron range in lead is $1.0 \mathrm{~mm}$, while is $0.6 \mathrm{~mm}$ in gold and tungsten. This means that secondary electrons in lead cam more efficiently escape the protector ant deposit in a surface dose. Besides, the beam hardening in lead is less pronounced than in gold or tungsten and, thus, more low energy photons deposit their energy to the surface layer.

The next matter studied was how the diameter of the protector influences the surface dose. Different behavior for gold and lead was noticed as Figs. 6-14(c) and 6-14(d) show. Figure 6-14(c) illustrates the surface dose influenced by gold protector decreases as protector diameter increases. Moreover, for $2.0 \mathrm{~cm}$ protector the surface dose is even lower than that of the open beam. The effect of tungsten protectors is similar. For lead protectors of different diameters the difference is slight (see Fig. 6-14(d)). This shows that the outer shell of the cylindrical protector is the source of the secondary electrons, because due to small electron ranges in gold, tungsten and lead, the electrons produced within the protector at a distance larger than the electron range are absorbed. Several authors have pointed out that increase in material thickness increases the amount of scattered electrons until it reaches the electron range in this material, while the additional to the CSDA thickness of materials does not increase the surface dose (Cardarelli et al. 1991). An increase in protector diameter increases the amount of secondary electrons that exit the protector due to increment of the outer shell volume. For gold and tungsten the increase of the number of secondary electrons does not compensate the surface dose decrease due to beam hardening. For lead, for which these two opposite effects seem to be in equilibrium, the surface dose does not depend significantly from the protector diameter.

A surface dose for tungsten protectors yielding the same attenuated field width $d_{c}$ is shown in figure 6-14(e). Similar tendencies of a surface dose increment are noticed for the gold and lead protectors as well. This figure shows that protector-to-surface distance plays more significant role than the protector diameter.

Figure 6-15 shows the surface dose dependence on the field size for beams attenuated by lead and gold protectors of $1.0 \mathrm{~cm}$ and $1.6 \mathrm{~cm}$ in diameter in rotational therapy. Three square field sizes were simulated with sides $5 \mathrm{~cm}, 7.5 \mathrm{~cm}$ and $10 \mathrm{~cm}$. At large fields the surface dose is larger when compared with small field sizes, including the open field. This means that the amount of backscattered particles increased significantly. According to Fig. 615 , the radiation, unaffected by protector, deposit the bigger part of surface dose. Jiang and 

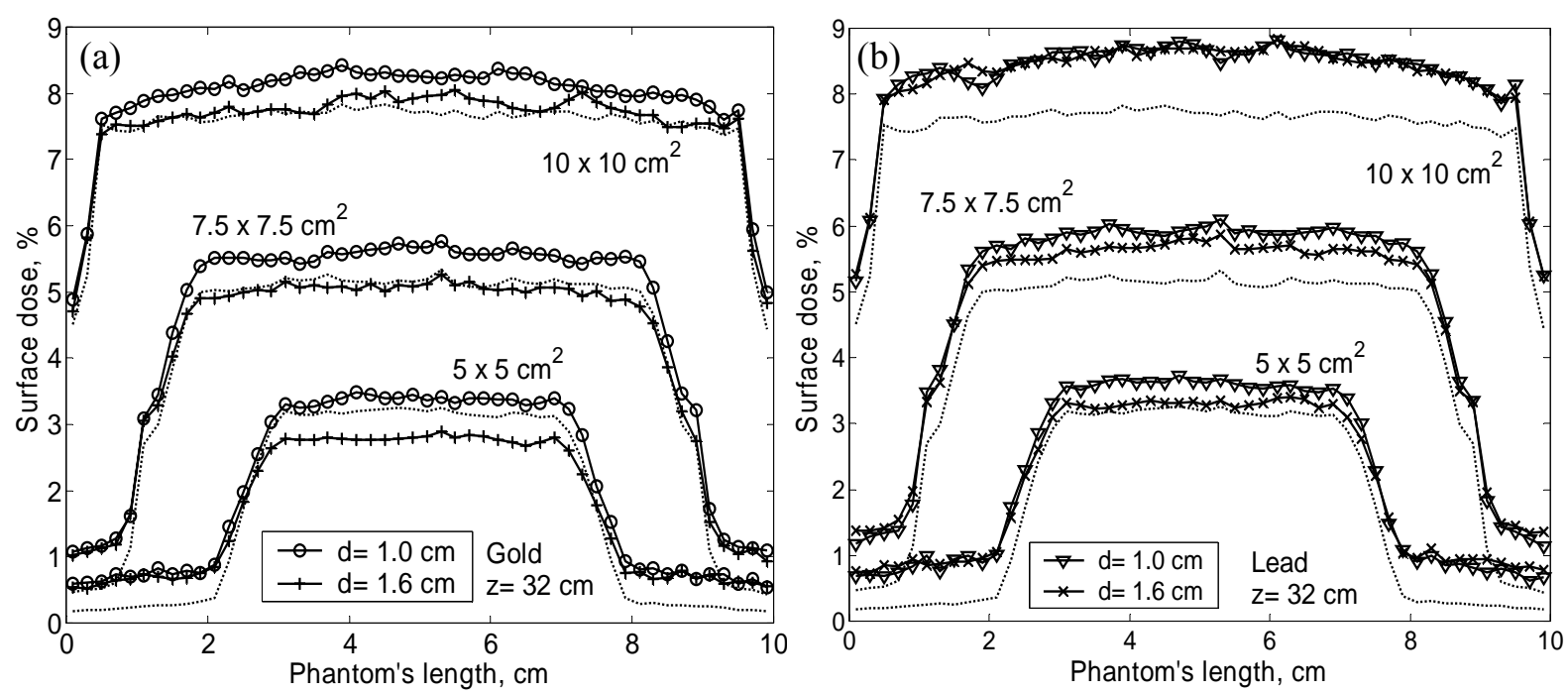

Fig. 6-15. Surface dose dependence on the field size at rotational irradiation with the beams attenuated by gold (a) and lead (b) protectors of 1.0 and $1.6 \mathrm{~cm}$ in diameter. The relative surface dose is expressed as percentage of open beam at the isocenter. The surface dose for open beam is also added.

Ayyangar (1998) showed that cerrobend compensator increases the contaminant electron surface dose by about $1 \%$ of the maximum (side of square field is $10 \mathrm{~cm}$ ). As field size increases, the scattered electrons and photons contribute more markedly the surface dose and the surface dose increases comparing the open beam surface dose. This enhancement can be ascribed to relative more scattered electrons from a protector that can reach the surface. The scatter angles exiting the protector are assumed to be relatively large (du Plessis and Willemse 2005).

The influence of the field size to the surface dose is much more pronounced than the effect of the protector diameter. The average surface dose values for different field sizes are presented in Table 6-8. The surface dose values due to protectors inserted in the beam are different regarding to the open beam surface dose for every field size. Let's analyze Fig. 615(a) first, where surface doses due to gold protector 1.0 and $1.6 \mathrm{~cm}$ in diameters for square fields of side $5 \mathrm{~cm}, 7.5 \mathrm{~cm}$ and $10 \mathrm{~cm}$ are presented. For $5 \times 5 \mathrm{~cm}^{2}$ field $1.0 \mathrm{~cm}$ protector's surface dose is higher by $0.2 \%$ (of the open beam at the isocenter) than that of open beam, while for $1.6 \mathrm{~cm}$ protector is lower by $0.4 \%$. For $7.5 \times 7.5 \mathrm{~cm}^{2}$ the surface dose of $1.0 \mathrm{~cm}$ protector is $0.4 \%$ higher than that of open field and of $1.6 \mathrm{~cm}$ protector approaches the magnitude of the open beam. For $10 \times 10 \mathrm{~cm}^{2}$ field the surface dose for both diameters of protectors are higher than of open beam. It means that the amount of scattered radiation from the protector increases. Additionally, due to big scatter angles of the electrons exiting the compensator more scattered electrons can reach the surface. Comparison of the differences in the surface doses of protectors of 1.0 and $1.6 \mathrm{~cm}$ in diameter for different field sizes shows 
TABLE 6-8. Average surface dose for square fields with side size of $5.0 \mathrm{~cm}, 7.5 \mathrm{~cm}$ and $10.0 \mathrm{~cm}$ for open beam and attenuated by gold and lead protectors 1.0 and 1.6 $\mathrm{cm}$ in diameter. The surface dose values are expresses as percentage of the dose at the isocenter of open beam for every field size, respectively.

\begin{tabular}{|c|c|c|c|c|c|}
\hline \multirow{3}{*}{$\begin{array}{c}\text { Size of } \\
\text { square field, } \\
\mathrm{cm}\end{array}$} & \multicolumn{5}{|c|}{ Average surface dose, $\%$} \\
\hline & \multirow{2}{*}{$\begin{array}{l}\text { Open } \\
\text { beam }\end{array}$} & \multicolumn{2}{|c|}{ Gold protector } & \multicolumn{2}{|c|}{ Lead protector } \\
\hline & & $\mathrm{d}=1.0 \mathrm{~cm}$ & $\mathrm{~d}=1.6 \mathrm{~cm}$ & $\mathrm{~d}=1.0 \mathrm{~cm}$ & $d=1.6 \mathrm{~cm}$ \\
\hline 5.0 & 3.2 & 3.4 & 2.8 & 3.6 & 3.3 \\
\hline 7.5 & 5.1 & 5.5 & 5.0 & 5.8 & 5.6 \\
\hline 10.0 & 7.7 & 8.1 & 7.8 & 8.5 & 8.5 \\
\hline
\end{tabular}

that this difference decreases as a field size increases. This may show that the lower surface dose due to beam hardening effect is "spoiled" by the increased scatter of secondary particles.

For lead the situation is similar: with increase of field size the difference between the open beam and attenuated beams surface dose increases. However, even for $5 \times 5 \mathrm{~cm}^{2}$ field the surface doses both for 1.0 and $1.6 \mathrm{~cm}$ are higher than that of open beam. The lower surface dose due to hardening effect is equilibrated by higher than for gold secondary electrons influence to the surface dose. With increase of the field the surface dose curve of 1.6 $\mathrm{cm}$ protector approaches that of $1.0 \mathrm{~cm}$. This means that that for $1.6 \mathrm{~cm}$ the increase in scattered electrons influence to the surface dose is in bigger extend than the beam hardening. For the protector of $1.6 \mathrm{~cm}$ in diameter photons scatter from the protector is more pronounced, as well.

Ratio $\mathbf{D}_{\mathbf{c}} / \mathbf{D}_{\mathbf{m a x}}$. A protector inserted in a beam during rotational radiotherapy effects also the dose outside the OAR. Tissues within the PTV, but close to the shielded region, will receive a lower dose than tissues further away, as they remain within the shadow of the protector for a larger fraction of the rotation time. A protector of higher density and larger diameter has bigger dose reduction outside the OAR, as we can see in Figs. 6-5 and 6-7, but also lower dose at the OAR is acquired. The successful treatment is associated with the ratio $\left(D_{c} / D_{\max }\right)$ of the dose at isocenter to the maximal dose outside the OAR. The lower this ratio is the better protector performance is achieved. The ratio $D_{c} / D_{\max }$ versus protector distance $d$ is shown in Fig. 6-16. 


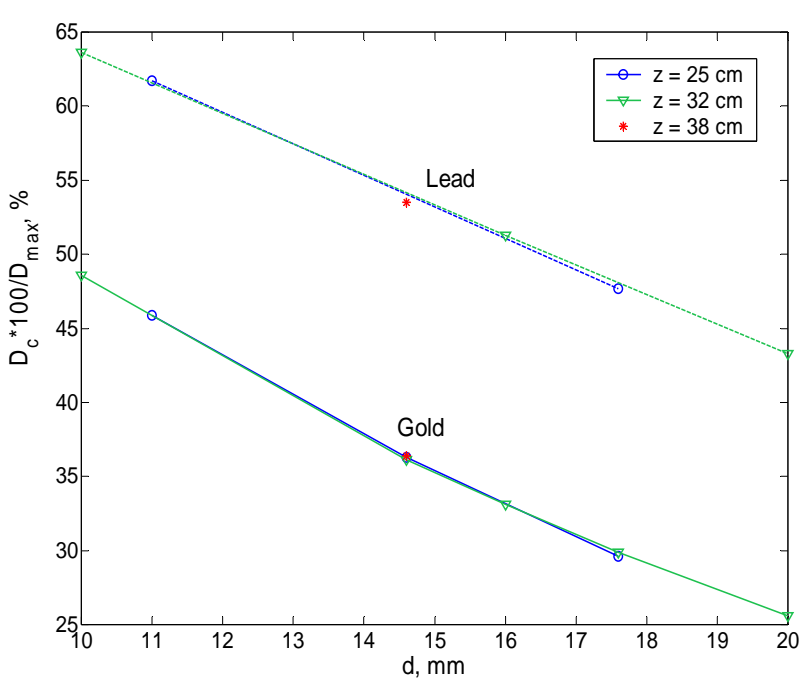

FIGURE 6-16. Ratio of maximum to minimum doses versus protector diameter.
Dose gradient beyond the OAR. Analysis of dose gradients for different protectors and their positions above the surface showed that protectors of higher density have steeper dose increase, as shown in Fig. 6-17(a). However, for protectors of the same attenuated field width $\mathrm{d}_{\mathrm{c}}$, the further the protector is placed from the irradiation source $(\mathrm{z}$ is smaller) the higher dose gradient the corresponding dose distribution has (see Fig 6-17(b)). This is a consequence of a smaller geometrical penumbra (Johnes and Cunningham 1983b). Generally, the distance between the maximum gradient points is approximately equal to the attenuated field width $d_{c}$.
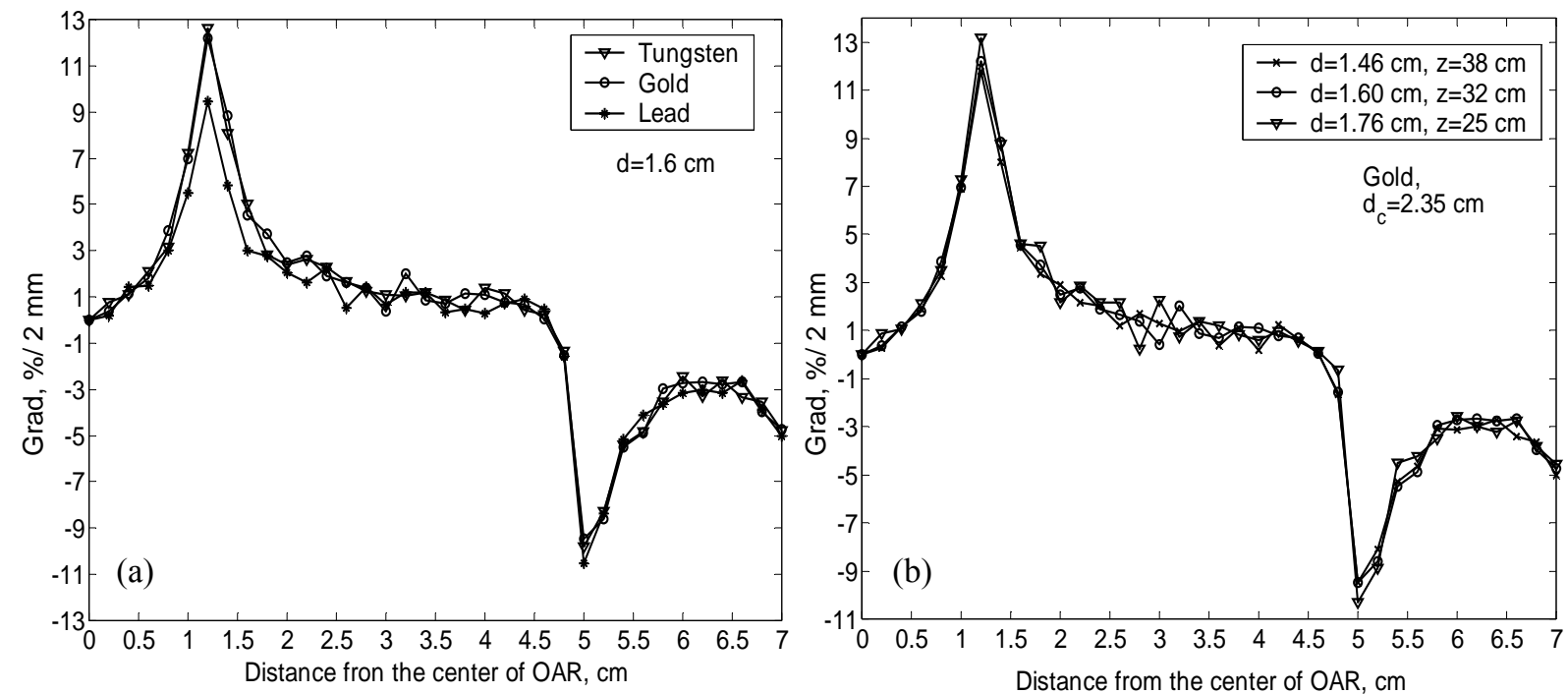

FIGURE 6-17. Dose gradients $\Delta \mathrm{D} / \Delta \mathrm{r}$ are shown for (a) protector of the same diameter and made of different materials and (b) for gold protectors yielding the same attenuated field widths $d_{c}$ that have different diameters and are placed at different distances from the irradiation source.

A study of the gradient as difference in dose between 2 neighboring voxels is insufficient, because in some cases two successive differences can be of approximately the same value, like in the case presented in Fig. 6-18(a), however the overall dose increase along $\mathrm{x} \mathrm{cm}$ is higher (Fig. 6-18(b)). For example, the dose increase from $1 \mathrm{~cm}$ to $2 \mathrm{~cm}$ from the center of the OAR is $3.3 \% / \mathrm{mm}, 3.0 \% / \mathrm{mm}$ and $2.7 \% / \mathrm{mm}$ for gold cylinder of $1.46 \mathrm{~cm}$ in 

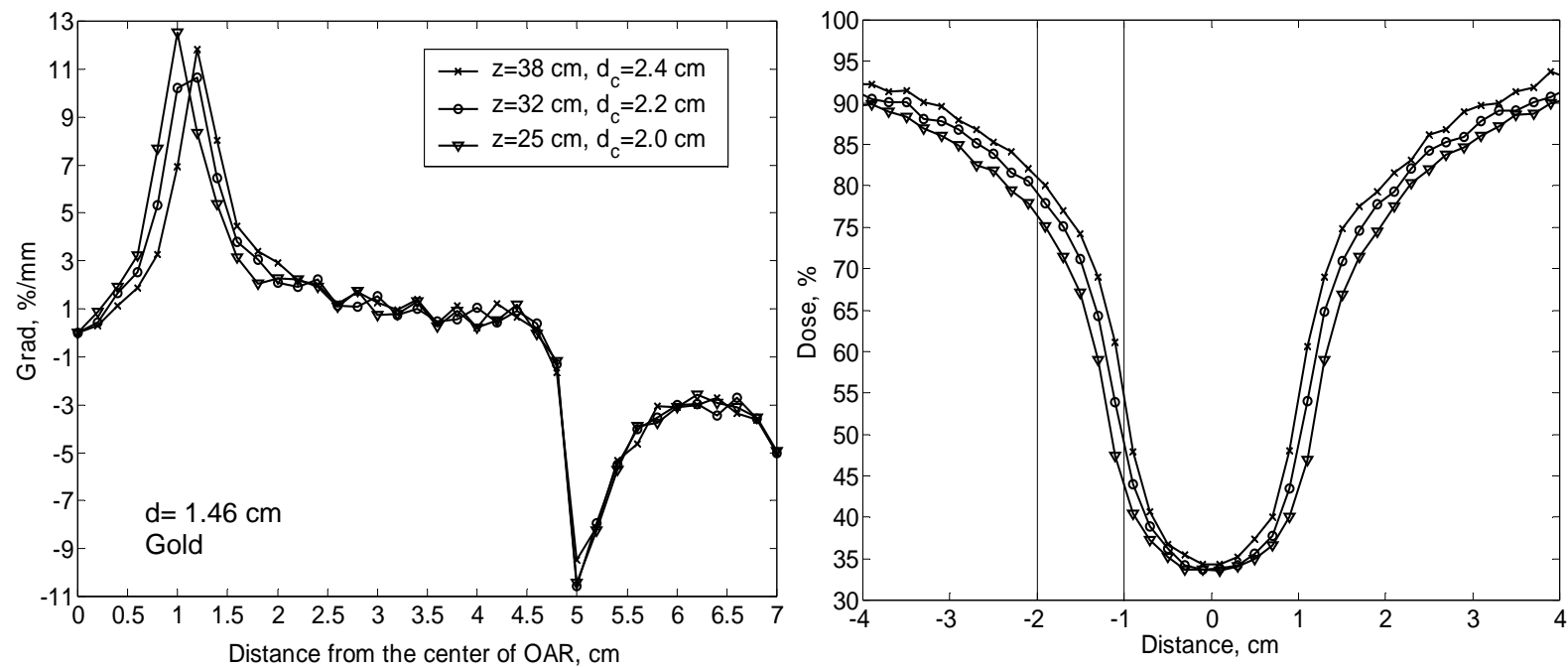

FIGURE 6-18. Illustration of the dose increase (gradient) between two successive voxels (a) and the corresponding dose profiles (b) for the gold protectors of $1.46 \mathrm{~cm}$ in diameter. The overall dose increase along the distance of $1 \mathrm{~cm}$ (from $1 \mathrm{~cm}$ to $2 \mathrm{~cm}$ from the center of the OAR) for the curve of the protector placed at $\mathrm{z}=25 \mathrm{~cm}$ is higher than that of $\mathrm{z}=38 \mathrm{~cm}$. However, this is not clearly seen in left figure (a).

diameter placed at $\mathrm{z}=38 \mathrm{~cm}, 32 \mathrm{~cm}$ and $25 \mathrm{~cm}$, correspondingly. In Fig. 6-18(a) the gradient has two nearly equal and high values for $1.6 \mathrm{~cm}$ protector placed at $32 \mathrm{~cm}$ from the isocenter.

\subsection{DISCUSSION AND CONCLUSIONS}

A comparison of simulated and measured attenuation curves gave entirely satisfactory results. The experimental and simulated data were found to be in a good agreement with a RMS error not higher than $2.1 \%$. The maximal discrepancy between the effective linear attenuation coefficients derived from measured and simulated attenuation curves was $4.7 \%$. This can be caused by the insufficient information about the chemical constituents of the curing agent of the Technon/Poly material for which the highest error was noticed. Inaccuracies in density measurements can also yield a systematic error in simulation data. Nevertheless, the MCRTS was proven to be a reliable tool for studies of the effect of the protectors on dose distributions in homogeneous phantoms.

Lead, as it was expected, provides superior protection, than cerrobend and brass do. The exploration of the attenuation properties of the studied materials showed that the best metalpolymer composite is Ecomass. Its effective linear attenuation coefficient is very close to that of lead. The other two materials of this group showed inferior protection properties than Ecomass, because their densities were significantly lower than expected. The reason could be in the processing procedures that these materials underwent. However, if the density provided 
is high (it can be achieved till $12 \mathrm{~g} / \mathrm{cm}^{2}$ ), the metal-polymer composites can rival the lead in the protection of radiosensitive tissues.

The second part of our studies showed that dose distributions resultant from a rotational irradiation with cylindrical protectors inserted in the megavoltage beam depend in a complicate way on all attenuator parameters, as we can see from Figs. 6-5 to 6-8. For the same protector material and z-coordinate, the dose at the center of the OAR decreases as protector diameter (and therefore attenuated field width) increases, as it shown in Fig. 6-5, because primary radiation and scatter contribution to the central part of the OAR from phantom are reduced. For the same protector material and diameter, dose at the center of the OAR decreases if z-coordinate of the protector increases, as it is illustrated by Fig. 6-6. Scatter from the attenuator decreases, as well as scatter contribution from the phantom does (because of larger attenuated field width at the isocenter). The effect of scattered photons at the center of the OAR is less pronounced, because they are absorbed prior to reach the center of the attenuated field. If the diameter and the z-coordinate are the same, the use of denser protector material leads to more pronounced decrease of primary radiation, as shown in Fig.67. Tungsten and gold have comparable protection that is better than of all other materials. Compton effect is the most predominant mode of interactions for $6 \mathrm{MV}$ photon beams, its cross-section is proportional to the density of the material and is independent of atomic number Z (Khan 1994). Both materials have very close densities (refer to Table 6-4). However, gold has slightly bigger absorption. It has higher atomic number than tungsten (79 versus 74) and, thus, increased production of photoelectrons and electron pairs that contribute to the absorption in addition to Compton electrons. Dose distributions, obtained with lead and Rad-block (tungsten powder filled nylon 6) protectors are similar as well. Although lead has slightly lower density it has higher atomic number. Cerrobend can not rival the above discussed materials in terms of protection. Finally, as shown in Fig. 6-8, for the same material and attenuated field width the dose at the center of the OAR decreases, if the protector's diameter increases, because of the increase in the primary beam attenuation.

Thus, concerning the optimal protectors for rotational radiation therapy, it was observed that gold and tungsten materials have the best beam attenuating properties. Nevertheless, gold and tungsten are expensive and hard to fabricate. Lead, which is commonly used and provides adequate protection, is toxic and hazardous to handle. Polymer-metal composites can be a good choice as lead alternatives. They are not hazardous, easy to handle and have relatively high density (till $12 \mathrm{~g} / \mathrm{cm}^{3}$ ). Other solution can be lead cylinder covered by a thin layer of 
higher density material, like gold. An improvement of the performance is shown in Fig. 6-13. The dose at the center of the OAR for protector, consisting of a lead core of $1.4 \mathrm{~cm}$ and a gold outer annulus of $2 \mathrm{~mm}$, is lower than that of pure lead of $1.6 \mathrm{~cm}$ in diameter. Moreover, the dose variation at the protected region is quite small. Additionally, uranium can be considered as a material for the protector.

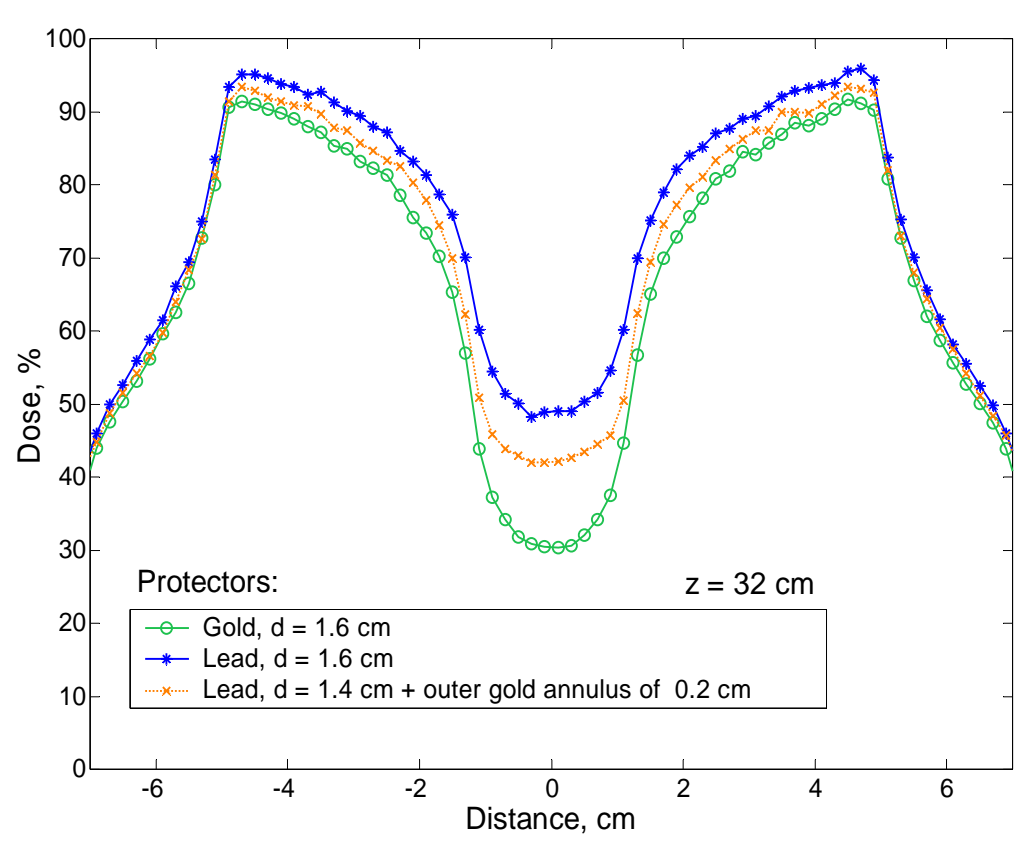

FIGURE 6-13. Comparison of dose distribution below the protector made of two materials $(1.4 \mathrm{~cm}$ lead core with outer $0.2 \mathrm{~cm}$ gold annulus) with distributions of pure lead and gold protectors of $1.6 \mathrm{~cm}$ in diameter.
There is quite a noticeable dependence of a dose at the center of the protected region from the field size. The values for larger fields are larger due to dose enhancement of inphantom scattered radiation, contributing to the dose. Due to increase in scatter contribution, the dose value increases with increasing field size for all the thicknesses that is in accordance with the investigations presented in the literature (El-Khatib et al.

1987, Weeks et al. 1988, Islam and Van Dyk 1995). This effect was extensively studied and some methods were proposed to include field size as parameter in equations for linear attenuation coefficient calculations for broad beam (Van Dyk 1986, du Plessis and Willemse 2003). In this work the dependence of the dose at the isocenter from the field size was parametrized with a linear function $\mathrm{D}_{\mathrm{c}}=\beta \cdot \mathrm{W}_{0}+\alpha$. The coefficient $a$, actually, shows a zero field size transmission at the center of the OAR. A close agreement was found between the values, received by an extrapolation of the data with linear regression analysis and the calculated primary beam transmission values (see Table 6-8).

The magnitude of scattered radiation contribution is illustrated by Fig. 6-11. There is a noticeable difference between the primary radiation curves and $\mathrm{MC}$ results, which include scattered radiation. The curves calculated, using spectral distribution of incident photons (i.e. including beam hardening effect) are slightly closer to the $\mathrm{MC}$ calculations. The difference in the location about the surface has much smaller influence on the dose at the center of the 
OAR than difference in the diameter. For gold protector of $\mathrm{d}=1.1 \mathrm{~cm}$ the difference in dose for $\mathrm{z}=25 \mathrm{~cm}$ and $\mathrm{z}=32 \mathrm{~cm}$ is $1.6 \%$, while for $\mathrm{d}=1.0 \mathrm{~cm}$ and $1.1 \mathrm{~cm}$ for $\mathrm{d}=32 \mathrm{~cm}$ it is $7.7 \%$.

For attenuators inserted into the beam, the scattered dose increases with increased attenuated thickness, but for thickness values of more than one mean-free path, the scattered dose decreases (Islam and Van Dyk 1995, Castellanos and Rosenwald 1998). This occurs because the scattered radiation generated near the entrance side of the absorber is attenuated by the larger thickness of the attenuator. Moreover, the number of scattered photons generated on the exit side of the attenuator is reduced due to the lower intensity of the primary photons. This was noticed also in our studies, that for gold and tungsten protectors that have mean-free path $1.1 \mathrm{~cm}$, the scatter contribution to the dose at the central axis of the OAR decreases as the protector's diameter increases in diameter from $1.0 \mathrm{~cm}$ to $2.0 \mathrm{~cm}$. The maximum for 1.1 $\mathrm{cm}$ was not noticed due to luck of data for this protector's diameter. For lead the scattered radiation dose increases with diameter, as the mean-free path for lead is $1.9 \mathrm{~cm}$. So, beyond this diameter's value the scattered photons contribution has to decrease. Unfortunately, due to lack of data, it was no possible to verify here. The scatter-to-primary dose ratio increases for all studied materials that show that the decrease of primary dose is much more pronounced than any increase in the dose due to scattered radiation contribution. This is in accordance with the Eq. 6-2, which shows that the first scatter is proportional to the thickness of the attenuator.

The relative contribution of scatter is increasing with the field size, as the results of other studies also show (El Khatib et al. 1986, Huang et al. 1986). In-phantom scatter contribution to the dose at a given point increases with the field size. It can be noticed that for small fields, such as with sides 5 and $7.5 \mathrm{~cm}$, the scatter is higher for smaller protector diameter for all protector's materials. It can be explained by the fact that the attenuated field size at the isocenter for smaller diameter protector is smaller and the in-phantom scattered photons can reach the central part of the shielded region. For $10 \times 10 \mathrm{~cm}^{2}$ field size, the phantom scatter is large enough to deposit a dose to the center of the shielded region both of $1.6 \mathrm{~cm}$ and $1.0 \mathrm{~cm}$ protectors. Another parameter that affects the dose contribution from the scattered photons is a filter-to-point of interest distance. The dose contribution from the scattered photons produced in the filter is greater for shorter distances (Huang et a. 1986, Islam and Van Dyk 1995). It should be also noted that since a scattered radiation represents only a small fraction of the contribution to the dose, it has a much larger percentage of uncertainty than the transmission factors, especially for small field sizes and thicknesses. 
High energy photon beams, which are used for treating deep-seated tumors, are characterized by a skin-sparing effect. However, secondary electrons generated in the patient or contaminating electrons produced outside the patient may reduce this effect. Surface dose is the result of electron contamination of the incident beam as well as backscattered radiation (both electrons and photons) from the medium. There has been a controversy as to the relative contribution of secondary electrons versus low energy scattered photons to the dose in the build- up region (Khan 1994). Several observations can be made regarding the surface dose in rotational therapy. The average values for field size $10 \times 10 \mathrm{~cm}^{2}$ (at $\mathrm{SID}=100 \mathrm{~cm}$ ) ranged from 7 to $9.5 \%$, that are generally lower that of the stationary field. The surface doses reported for a one gantry irradiation are in the range of 29 to $30 \%$ of the maximal dose of a 10 $\times 10 \mathrm{~cm}^{2} 6 \mathrm{MV}$ photon beam $(\mathrm{SSD}=90 \mathrm{~cm})$ (Jiang and Ayyangar 1998). The open field surface dose measured by Davis and Reiner (1995) was $41 \%$ for $15 \times 15 \mathrm{~cm}^{2}$ field at $\mathrm{SSD}=$ $100 \mathrm{~cm}$ for $6 \mathrm{MV}$ photon beam, which reduces till $6.5 \%$ for blocked by narrow block $(3 \mathrm{~cm}$ in width at SSD). A phantom surface dose is affected primarily by the protector-surface distance and field size (Ciesielski et al. 1989, Cardarelli et al. 1991, Mellenberg 1995, Kim et al. 1998) because of the large electron emission angles for the electrons emitted from high atomic number filters (Nilsson 1985, du Plessis and Willemse 2005). For ${ }^{60}$ Co source, a medium atomic number will give the lowest surface dose. For low atomic number the incoherent cross section dominates and a large forward directed electron contribution is obtained. For higher atomic numbers the influence of multiple scattering will reduce the absorbed dose at the center of the field. However, at very high atomic numbers the increasing photoelectric cross section will dominate. For high energies the contribution of pair production is also added (Nilsson 1985). As was shown by Mellenberg (1995) both gypsum (material with low density and effective atomic number) and lead (with opposite properties) have little effect on surface and dose built-up region doses when placed $40 \mathrm{~cm}$ away from the patient's surface. This effect can be explained by big scatter angles of the secondary electrons (du Plessis and Willemse 2005). The electron scatter angle $\varphi$ can be derived from the conservation of energy and conservation of momentum at the collision of a photon with a stationary electron and is given by

$$
\varphi=\sin ^{-1}\left\{h v^{\prime} \sin \theta \frac{\sqrt{1-\beta^{2}}}{0.511 \beta}\right\}, \quad \sqrt{1-\beta^{2}}=\frac{0.511}{E+0.511} .
$$

At $6 \mathrm{MV}$ photons scatter angle $\theta \leq 45$ and the electrons would scatter between $27^{\circ}$ and $90^{\circ}$ in the compensator. A few percent of the electrons are produced from photon scatter 
larger than $45^{\circ}$ that would scatter in the forward direction in the compensator. Besides, the initial distribution of the electrons is perturbed by single and multiple scatterings resulting in increased divergence of the electrons. This effect depends on the photon energy and the atomic number of the material (Hine 1951).

Thus, the lower surface dose, when gold and tungsten protectors are used, is due to more pronounced beam hardening. In the case of lead absorber, lower energy photons and electrons contribute to higher surface dose. This proves to be true also because the surface dose for $10 \mathrm{~mm}$ protectors, both for gold and tungsten, is higher than for 16 and $20 \mathrm{~mm}$ protectors in diameter placed at the same distance from the isocenter, as it can be see in Fig. 6-13(c). The beam hardening has a larger effect on the surface dose than on the dose at $10 \mathrm{~cm}$ depth. Figure 6-13(e) illustrates that protector-to-surface distance affects the surface dose more than increase in protector diameter.

The protector (or other beam filters) has three major effects on contaminant electrons, which are (i) the absorption of the contaminant electrons generated by the treatment head above the protector (that was not considered in a present work), (ii) the generation of new contaminant electrons by the interactions of photons with the protector and (iii) the selfabsorption in the protector generated electrons. As the protector is of cylindrical shape, the increase in protectors' diameter increases its volume, where secondary electrons are generated, although in the internal core of protector the generation and self-absorption are in equilibrium. The influence of the compensator on the contaminant electron surface dose is small. The scattered photons have large spatial frequency. Compensator scattered photons form a uniform low fluence background at the surface, thus almost have no effect on a surface dose (Jiang and Ayyangar 1998). The effect of the blocking tray, which houses the compensator of protector during treatment, was not studied. This effect was reported to be significant and in some cases exceed the effect of compensator itself (Cardarelli, Rao and Cail 1991).

The higher the density of the protector is and the larger the diameter is, the bigger dose reduction appears outside the OAR, as well. The ratio $D_{c} / D_{\max }$ decreases with increase of diameter and density and, therefore, protector's performance improves. In other words, the lower transmission protector provides lower minimum to maximum dose ratio. The dose homogeneity outside the OAR, opposite, is worsening. The dose homogeneity can be improved by use of beam shapers, as it was shown both by experimental (Danciu 2001) and by simulation (Ivanova et al. 2005) studies. 
An analysis of penumbra showed the dose distribution of a beneficial desired feature, such as steep dose increase just outside the protected region, is achievable with higher density protectors, placed further from the irradiation source.

In the studies presented in this chapter, the isocenter coincided with the center of the OAR. The location of the protector regarding to the isocenter (accordingly to the location of the OAR) is not of big importance and don't have effect on the properties of the protected region, as shown in previous chapter, so the results of the study presented can be extended to any protector located arbitrarily regarding the isocenter. The point source model used is a good approximation as long as the dose points at the beam edge do not play an important role.

So, concluding, we can say that in order to achieve more extensive protection of the OAR, a protector of high density and bigger diameter are preferable. In the case of cylindrical protector, however, increase in diameter leads to increase of projected shadow, which is not always desirable. So, the solution can be to use larger diameter protector, placed closer to the phantom. Scatter contribution to the center of the OAR from protector is negligible. Surface dose is increasing not more than by $1 \%$ of the dose of the open beam, but surface dose is not so high, even in the case of protector placed at $17.5 \mathrm{~cm}$ from the phantom surface. 


\section{CONCLUSIONS AND FUTURE WORK DIRECTIONS}

\subsection{CONCLUSIONS}

A framework of the conformal dose delivery by means of beam modifying devices presented in this thesis is a step forward towards an application of a technique that combines both good dose conformation properties and cost effectiveness. Intensity modulation by means of beam modifying devices, based on the principle of the gravity-oriented filters, has been presented and investigated.

The principles of the field shaping similar like by the gravity-oriented devices were preserved, but the initial orientation of the beam modifying devices was arbitrary, while their motion was motor-driven. The beam modifying devices were divided in two groups: protectors and beam shapers; and mathematical description of the motion and of the parameters calculation scheme was developed separately for every group. The concept, how to model the protector, has already been contained in the idea of the protection itself, i.e. the protector has to cast shadow to the OAR for every gantry angle. Two methods of beam shapers calculations were worked up. The use of the first one leads to a proper material for the shapers of a defined diameter, and the second method, on the contrary, searches for an optimal shaper's diameter of a given material. The first method requires an initial guess of the 
shaper's diameter that can lead to a certain appropriate material, although their number is limited. The second method is more flexible and yields good results in term of uniformity both for monoenergetic and polyenergetic beams, as a verification of the method showed.

The software application for the computations of the geometrical setup and dimensions of the beam modifying devices as well as the user interface to facilitate the introduction of the input parameters were developed. The verification of the software tool showed good agreement between experimental and simulation data, with discrepancies of less than $3 \%$. The software tool developed was proven to be a reliable tool for studies of beam modulation with beam modifying devices.

Conceptual studies of intensity modulation by means of beam modifying devices showed high degree of dose conformity, in the case of their use to the spinal cord protection, using both solid-geometry and voxel-based neck models. The studies of the intensity modulation showed that the lead cylinder of $1.6 \mathrm{~cm}$ in a diameter, introduced in the beam, results to a significant dose reduction. And solely within the outer annulus of $1 \mathrm{~mm}$ the dose is beyond the tolerance level due to characteristic rapid increases at the border of the cylindrical OAR. Highly uniform dose of order of less than $1 \%$ of the average dose at the PTV can be assured by means of beam shapers used in the assembly with the protector. Results demonstrated that the protection of the OAR is not affected by the shapers, while their use effectively improves dose uniformity. Simulation of the rotational irradiation with the beam modulated with the same protector, but shapers made of different materials: iroco wood and polystyrene, demonstrated a similar pattern of dose distributions, with a slightly higher dose in the PTV when using iroco shapers. More uniform dose in the PTV for the case of the iroco shapers was also noticed.

Experimental measurements of the beam attenuation by a number of materials used for a protection of vital organs showed that lead, as was expected, provides superior protection, than cerrobend and brass do. The best attenuation properties among the studied metal-polymer composites Ecomass material presented, with effective linear attenuation coefficient approaching to that of lead. If the density provided is high (it can be achieved till $12 \mathrm{~g} / \mathrm{cm}^{2}$ ), the metal-polymer composites can rival the lead in the protection of normal tissues during tumor irradiation. In simulation studies of the effect of the protector's parameters on the dose distribution in rotational therapy, gold and tungsten showed the best protection properties. Lead has inferior protection comparing to gold and tungsten, but by providing the appropriate thickness can effectively decrease the irradiation dose to the healthy tissues. Protector of a 
diameter that is approximately equal to one mean-free path (i.e., $1 / \mu)$ ensures sufficient protection. The synchronous protection during gantry motion in rotational therapy implies the protector to be an appropriately scaled copy of the OAR. So, the actual size of the protector depends on the distance from the source. Thus, by increasing this distance, the thickness of the protector increases. But skin sparing effect must not be lost. The studies showed that a lead protector yields the highest surface dose. Nevertheless, it is relatively low (less that 9.5 $\%$ ) even for the shortest studied protector-to-surface distance of $17.5 \mathrm{~cm}$. It was found also that the protector-to-surface distance affects the surface dose more than the protector diameter. The ratio of the dose at the center of the OAR to the maximal dose decreases with the increase of the diameter and the density and, therefore, the protector's performance improves. Steeper dose increase beyond the protected region is achievable with higher density protectors, placed further from the irradiation source.

Thus, concerning the optimal protectors for rotational radiation therapy, it was observed that gold and tungsten materials have the best beam attenuating properties. Nevertheless, gold and tungsten are expensive and hard to fabricate due to high melting temperature. Lead that is commonly used and provides adequate protection is toxic and hazardous to handle. Polymermetal composites can be a good choice as lead alternatives. They are not hazardous, easy to handle and have relatively high density.

Besides, some aspects of the MCRTS were verified in the work. A comparison of simulated and measured attenuation curves in different materials gave entirely satisfactory results. The experimental and simulated data demonstrated good agreement with a RMS error not higher than $2.1 \%$. The maximal discrepancy between the effective linear attenuation coefficients derived from measured and simulated attenuation curves was $4.7 \%$. Additional verification was performed together with the verification of the developed software tool and, as reported above, the observed discrepancies between simulated and literature data were less than $3 \%$.

Intensity modulation with beam modifying devices combines advantages of IMRT with compensators, such as time invariance of physical compensator, simplicity of treatment delivery and quality assurance, use of inexpensive materials, fine intensity modulation as well as of dynamic IMRT techniques, such as continuously varying intensity modulation and no need to change absorbers for each gantry orientation. Advantages of using this technique include also comprehensive and adequate protection at every gantry angle and uniform dose in PTV. Due to these gains it could be the preferred choice in many cases. 


\subsection{FUTURE WORK}

The future work directions that extend the scope of the above presented thesis could include:

(1) Extensive studies of the appropriate use of the beam modifying devices for treatment of other organs, such as cervix, prostate, esophagus etc. cancers.

(2) Solid-geometry protectors used in the current work can be replaced by more conformal protectors, corresponding to the $\mathrm{CT}$ images, keeping in mind, however, that they can be easy cast. Possible solution of this task can be protector construction in slice-by-slice manner.

(3) To create an auxiliary software tool, guiding a doctor in a decision making about the choice of appropriate protector for desired size of the OAR, distance between OAR and PTV, ratio of the dose at the OAR to the dose at the PTV and, finally, the distance of the protector from the source. The rich simulations data regarding the rotational irradiation of the cylindrical phantom with a single protector inserted into the beam can be used for derivation of geometrical and physical parameters of protectors that satisfy the dose prescription and setup requirements. 


\section{BIBLIOGRAPHY}

Barth N.M. (1990), “An inverse problem in radiation therapy”, Int. J. Radiat. Oncol. Biol. Phys. 18, 425-431.

Beavis A.W. (2004), “Is tomotherapy the future of IMRT?”, Br. J. Radiat. 77, 285-295.

Bentzen S.M. (2005), "Radiation therapy: Intensity modulated, image guided, biologically optimized and evidence based”, Radiother. Oncol. 77, 227-230.

Berger M.J., Coursey J.S., Zucker M.A. and Chang J. (2005), ESTAR, PSTAR, and ASTAR: Computer Programs for Calculating Stopping-Power and Range Tables for Electrons, Protons, and Helium Ions (version 1.2.3). [Online] Available: http://physics.nist.gov/Star [2006, October 16]. National Institute of Standards and Technology, Gaithersburg, MD. Material composition data: http://physics.nist.gov/cgi-bin/Star/compos.pl

Birkhoff G.D. (1940), “On drawings composed of uniform straight lines”, J. Mathematique 19, 221236.

Bliznakova K., Kolitsi Z. and Pallikarakis N. (2004), “A Monte Carlo based radiotherapy simulator”, Nucl. Instr. Methods B 222, 445-461.

Bliznakova K., Bliznakov Z. and Buliev I. (2006),“Monte Carlo radiotherapy simulator implemented on a distributed system", $5^{\text {th }}$ European Symposium on Biomedical Engineering ESBME 2006, July 79, Patras, Greece.

Bortfeld T., Bürkelbach J., Boesecke and Schlegel W. (1990), "Methods of image reconstruction from projections applied to conformation radiotherapy”, Phys. Med. Biol. 35(10), 1423-1434.

Bortfeld T.R., Kahler D.L., Waldron T.J. and Boyer A.L. (1994), "X-ray field compensation with multileaf collimators”, Int. J. Radiat. Oncol. Biol. Phys. 28, 723-730. 
Bortfeld T.R. and Boyer A.L. (1995), "The Exponential Radon Transform and projection filtering in radiotherapy planning”, Int. Journal of Imaging Systems and Technology 6, p.62-70.

Bortfeld T. (2006), “IMRT: a review and preview”, Phys. Med. Biol. 51, R363-R379.

Boyer A.L., Geis P., Grant W. et al. (1997), "Modulated beam conformal therapy for head and neck tumors”, Int. J. Radiat. Oncol. Biol. Phys. 39, 227-236.

Brady L.W., Kramer S., Levitt S.H. et al. (2001), "Radiation Oncology: Contributions of the United States in the last years of the 20th century", Radiology 219, 1-5.

Brahme A., Roos J.E. and Lax I. (1982), "Solution of integral equation encountered in rotat. therapy", Phys. Med. Biol. 27, p.1221-1229.

Brahme A. (1987), "Design principles and clinical possibilities with a new generation of radiation therapy equipment: A review”, Acta Oncol. 26, 403-412.

Brahme A. (1988), "Optimization of stationary and moving beam radiation therapy techniques”, Radiother. Oncol. 12, 129-140.

Bratengeier K. (2005), “2-step IMAT and 2-step IMRT: a geometrical approach”, Med. Phys. 32(3), 777-785.

Bratengeier K., Pfreundner L. and Plentje M. (2000), "Radiation techniques for head and neck tumors”, Radiother. Oncol. 56, 209-220.

Braunstein M. and Levine R.Y. (2000), "Optimum beam configurations in tomographic intensity modulated radiation therapy”, Phys. Med. Biol. 45, 305- 328.

Cardarelli G.A., Rao S.N. and Cail D. (1991), "Investigation of the relative surface dose from Lipowitz-metal tissue compensators for 24- and 6-MV photon beams”, Med. Phys. 18(2), 282-287.

Carol M.P. (1995), "Peacock ${ }^{\mathrm{TM}}$ : A system for planning and rotational delivery of Intensity-Modulated fields”, Int. J. Imaging Systems Technol. 6, p. 56-61.

Casebow M.P. (1990), “A modified synchronous shielding technique in rotation therapy”, Br. J. Radiol. 63, 482-487.

Chang S.X., Cullip T.J., Deschesne K.M. et al. (2004), “Compensators: An alternative IMRT delivery technique”, J. Appl. Clin. Med. Phys. 5(3), 15-36.

Chiu C.-H. and Spirou S.V. (2001), "Inverse planning algorithms for external beam radiation therapy", Med. Dosim. 26(2), 189- 197.

Chui C.S., Chan M.F., Yorke E. et al. (2001), "Delivery of intensity-modulated radiation therapy with conventional multileaf collimator: comparison of dynamic and segmental methods”, Med. Phys. 28, 2441-2449. 
Ciesielski B., Reinstein L.E., Wielopolski L. et al. (1989), "Dose enhancement in buildup region by lead, aluminum, and lucite absorbers for 15 MVp photon beam”, Med. Phys. 16(4), 609-613.

Convery D.J. and Rosenbloom M.E. (1992), "The generation of intensity-modulated fields for conformal radiotherapy by dynamic collimation”, Phys. Med. Biol. 37(6), 1359-1374.

Cormack A.M. (1987), “A problem in rotational therapy with x-rays”, Int. J. Radiat. Oncol. Biol. Phys. 13, p. 623-630.

Cormack A.M. and Cormack R.A. (1987), "A problem in rotation therapy with X-rays: dose distributions with an axis of symmetry”, Int. J. Radiat. Oncol. Biol. Phys. 13, 1921-1925.

Cormark R.A. (1998), “Beam profiles for x-ray rotation therapy”, Med. Phys. 25(6), 879-884.

Cotrutz C., Kappas C. and Webb S. (2000), "Intensity modulated arc therapy (IMAT) with centrally blocked rotational fields”, Phys. Med. Biol. 45, 2185-2206.

Coutard H. (1934), “Principles of x-ray therapy of malignant disease”, Lancet 2, 1-8.

Crooks S.M., Wu X., Takita C. et al. (2003), “Aperture modulated arc therapy”, Med. Phys. 48, 13331344.

Danciu C. (2001), "Development and application of new rotational radiotherapeutic techniques using gravity oriented absorbers and films”, Ph. D. thesis, University of Patras.

Danciu C. and Proimos B. (1997), “'Photomodelling': a simple conformal radiotherapy technique, modeling gravity oriented absorbers and photocopying them in the patient”, Med. Biol. Eng. Comput. 35(2), 1029. Proceedings of the World Congress on Medical Physics and Biomedical Engineering, Nice, France, 14-19 September (1997).

Danciu C. and Proimos B. (1999), "Gravity oriented absorbers in conformal radiotherapy for cervix cancer”, Radiother. Oncol. 51(1), S38-146. Proceeding of the 5th Biennial ESTROMeeting on Physics for Clinical Radiotherapy, April 6-11, 1999, Göttingen, Germany.

Davis J.B. and Reiner B (1995), "Depth dose under narrow shielding blocks: a comparison of measured and calculated dose”, Radiother. Oncol. 34, 219-227.

Davisson C.M and Evans R.D. (1952), “Gamma-ray absorption coefficients”, Rev. Mod. Phys. 24, 79107.

De Gersem W., Claus F., DeWagter C. et al. (2001), "Leaf position optimization for step-and-shoot IMRT”, Int. J. Radiat. Oncol. Biol. Phys. 51, 1371-1388.

Djordjevich A., Bonham D.J., Hussein E.M.A. et al. (1989), "Optimal design of radiation compensators”, Med. Phys. 17 (3), 397-404.

Dobbs J., Barrett A. and Ash D. (1999), "Practical radiotherapy planning”, $3^{\text {rd }}$ edition (Arnold, London), Chapters 6-8. 
du Plessis F.C.P. and Willemse C.A. (2003), "Monte Carlo calculation of effective attenuation coefficients for various compensator materials”, Med. Phys. 30(9), 2537- 2544.

du Plessis F.C.P. and Willemse C.A. (2005), "Radiological properties of wax-gypsum compensator material”, Med. Phys. 32(5), 1246- 1255.

Eisbruch A., Marsh L.H., Martel M.K. et al. (1998), "Comprehensive irradiation of head and neck cancer using conformal multisegmental fields: assessment of target coverage and noninvolved tissue sparing”, Int. J. Radiat. Oncol. Biol. Phys. 41, 559-568.

El-Khatib E.E., Podgorsak E.B. and Pla C. (1986), "The effect of lead attenuators on dose in homogeneous phantoms”, Med. Phys. 13(6), 928-935.

El-Khatib E.E., Podgorsak E.B. and Pla C. (1987), "Broad beam and narrow beam attenuation in Lipowitz’s metal”, Med. Phys. 14(1), p.135- 139.

El-Khatib E.E., Podgorsak E.B. and Pla C. (1988), "Calculation of dose in homogeneous phantoms for partially attenuated phantom beam”, Med. Phys. 15(2), 145-150.

Engler M.J., Herskovic A.M. and Proimos B.S. (1984), "Dosimetry of rotational photon fields with gravity-oriented eye blocks”, Int. J. Radiat. Oncol. Biol. Phys. 10, 431-438.

Ezzell G.A., Orton C.G. and Maughan R.L. (1987), "Practical aspects of transmission cord blocks in radiotherapy”, Med. Phys. 14(3), 400- 405.

Fiorino C., Lev A., Fusca M. et al. (1995), "Dynamic beam modulation by using a single computercontrolled absorber”, Phys. Med. Biol. 40, 221-240.

Fraass B.A. (1995), “Development of conformal radiation therapy”, Med. Phys. 22, 1911-1121.

Galvin J. M., Chen X.-G. and Smith R. M. (1993), "Combining multileaf fields to modulate fluence distributions”, Int. J. Radiat. Oncol. Biol. Phys. 27, 697-705.

Galvin J.M. (2006), “Alternative methods for Intensity-Modulated Radiation Therapy inverse planning and dose delivery”, Semin. Radiat. Oncol. 16, 218- 223.

Grubbe E. H. (1933), “Priority in the therapeutic use of x-rays”, Radiology 21, 1212-1217.

Hine G.J. (1952), "Scattering of secondary electrons produced by gamma rays in materials of various atomic numbers”, Phys. Rev. 82, 755-756.

Holmes T.W. and Mackie T. R. (1994) "A comparison of three inverse treatment planning algorithms”, Phys. Med. Biol. 39, 91-106.

Huang P.H., Chin L.M. and Bjärngard B.E. (1986), "Scattered photons produced by beam modifying devices”, Med. Phys. 13(1), 57-63. 
Hubbell J.H. and Seltzer S.M. (2004), Tables of X-Ray Mass Attenuation Coefficients and Mass Energy-Absorption Coefficients (version 1.4). [Online] Available: http://physics.nist.gov/xaamdi [2006, October 16]. National Institute of Standards and Technology, Gaithersburg, MD.

Huda W. and Slone R. M. (1995), “Review of radiologic physics”, Williams and Wilkins, Baltimore.

Hunt M.A., Zelefsky M.J., Wolden S. et al. (2001), “Treatment planning and delivery of Intensitymodulated radiation therapy for primary nasopharynx cancer”, Int. J. Radiat. Oncol. Biol. Phys. 49, 623-632.

ICRU report 50 (1993), "Prescribing, recording and reporting photon beam therapy", International Commission on Radiation Units and Measurements, Bethesda, MD.

Intensity Modulated Radiation Therapy Collaborative Working Group (2001), "Intensity-modulated radiotherapy: Current status and issues of interest”, Int. J. Radiat. Oncol. Biol. Phys. 51, 880-914.

Islam M.K. and Van Dyk J. (1995), "Effect of scatter generated by beam-modifying absorbers in megavoltage photon beams”, Med. Phys. 22(12), 2075-2081.

Ivanova T., Bliznakova K. and Pallikarakis N. (2005), "Studies of field shaping with "omoiotheta" beam modulators in rotational radiation therapy", The 3rd European Medical \& Biological Engineering Conference, IFMBE Proceedings 11(1), 1714-1717.

Ivanova T., Bliznakova K. and Pallikarakis N. (2006), "Simulation studies of field shaping in rotational radiation therapy”, Med. Phys. 33, 4289-4298.

Jabbari S., Kim H.M., Feng M. et al. (2005), "Matched case-control study of quality of life and xerostomia after intensity-modulated radiotherapy or standard radiotherapy for head-and-neck cancer: initial report”, Int. J. Radiat. Oncol. Biol. Phys. 63, 725-731.

Jena R., Luhana F., Brooke S.L. et al. (2004), “Conformal Rotation Therapy with central axis beam block is a feasible alternative to Intensity-modulated radiotherapy for chordomas of the cervical spine”, Clin. Oncol. 16, 449-456

Jiang S.B. and Ayyangar K.M. (1998), "On compensator design for photon beam intensity-modulated conformal therapy”, Med. Phys. 25(5), 668- 675.

Johns H.E. and Cunningham J.R. (1983), "The physics of radiology”, Charles C Thomas publisher, Springfield, USA.

Jursinic P.A., Podgorsak M.B. and Paliwal B.R. (1994), "Implementation of a three-dimensional compensation system based on computed tomography generated surface contours and tissue inhomogeneities”, Med. Phys. 21(3), 357-365.

Källman P., Lind B., Elköf A., Brahme A. (1988), "Shaping of arbitrary dose distributions by dynamic multileaf collimation”, Phys. Med. Biol. 33(11), 1291-1300.

Keall P., Zavgorodni S., Schmidt L. et al. (1997), “Improving wedged field dose distributions”, Phys. Med. Biol. 42, 2183-2192. 
Keall P.J. and Williamson J.F. (2003), "Clinical evidence that more precisely defined dose distributions will improve cancer survival and decrease morbidity", Letter to the Editor, Med. Phys. 30(6), 1281-1282.

Keller-Reichenbecher M.A., Bortfeld T., Levegrun S. et al. (1999), "Intensity-Modulation with the 'step and shoot' technique using a commercial MLC: a planning study”, Int. J. Radiat. Oncol. Biol. Phys. 45, 1315-1324.

Khan F.M, Sewchand W, Lee J. and Williamson J.F. (1980), "Revision of tissue-maximum ratio and scatter-maximum ratio concepts for cobalt 60 and higher energy x-ray beams”, Med. Phys. 7(3), 230237.

Khan F.M. (1994), “The physics of radiation therapy”, $2^{\text {nd }}$ ed. (Williams \&Wilkins, Baltimore, USA).

Kim S., Liu C.R., Zhu T.C and Palta J.R (1998), "Photon beam skin dose analyses for different clinical setups”, Med. Phys. 25(6), 860-866.

Kooy H.M. and Barth N.M. (1990), “The verification of an inverse problem in radiation therapy”, Int. J. Radiat. Oncol. Biol. Phys. 18, 433-439.

Lax I. and Brahme A. (1982), "Rotation therapy using a novel high- gradient filter”, Radiology 145, 473- 478.

Levy L.B., Waggener R.G., McDavid W.D. et al. (1974), "Experimental and calculated bremsstrahlung spectra from a 25-MeV linac and 19-MeV betatron”, Med. Phys. 1(2), 62-67.

MacKenzie M.A. and Robinson D.M. (2002), "Intensity modulated arc deliveries approximated by a large number of fixed gantry position sliding window dynamic multileaf collimator fields”, Med. Phys. 29(10), 2359-2365.

Mackie T.R., Holmes T., Swerdloff S. et al. (1993), “Tomotherapy: A new concept for the delivery of dynamic conformal radiotherapy”, Med. Phys. 20, 1709-1719.

Mackie T.R., Balog J., Ruchala K. et al (1999), “Tomotherapy”, Semin. Radiat. Oncol. 9(1), p.108117.

Mackie T.R. (2006), “History of Tomotherapy” Review, Phys. Med. Biol. 51, R427-R453.

Mageras G.S., Mohan R., Burman C. et al. (1990), "Compensators for three-dimensional treatment planning”, Med. Phys. 18(2), 133-140.

Martel M.K., Eisbruch A., Lawrence T.S. et al. (1997), "Spinal cord dose from standard head and neck irradiation: implications for 3D treatment planning”, Radiother. Oncol. 47, 185-189.

Mavroidis P., Lind B.K., Van Dijk J. et al (2000), "Comparison of conformal radiation therapy techniques within the dynamic radiotherapy project 'Dynarad” ”, Phys. Med. Biol. 45, 2459-2481.

Mellenberg D.E. (1995), “Dose behind various immobilization and beam-modifying devices”, Int. J. Radiat. Oncol. Biol. Phys. 32(4), 1193-1197. 
Meyer J., Mills J.A., Haas O.J. et al. (2000), “Some limitations in the practical delivery of intensity modulated radiation therapy”, Br. J. Radiol. 73, 854-863.

Miles E.A., Clark C.H., Guerrero Urbano M.T. et al. (2005), “The impact of introducing intensity modulated radiotherapy into routine clinical practice”, Radiother. Oncol. 77, 241-246.

Mohan R., Chui C. and Lidofsky L. (1985), "Energy and angular distributions of photons from medical linear accelerators”, Med. Phys. 12(5), 592-597.

Mohan R. Wang X. Jackson A. et al. (1994), "The potential and limitations of the inverse radiotherapy technique”, Radiother. Oncol. 32, 232-248.

Mota H.C., Vijayakumar S., Sibata C. et al. (1988), "Posterior spinal cord block: A dosimetric study", Radiology 168, 859-861.

Nakagawa K., Fukuhara N. and Kawakami H. (2005), “A packed building block compensator (TETRIS-RT) and feasibility for IMRT delivery”, Med. Phys. 32 (7), 2231-2235.

Nilsson B (1985), "Electron contamination from different materials in high energy photon beams", Phys. Med. Biol. 30, 139-151.

Oelfke U. and Bortfeld T. (1999), "Inverse planning for x-ray rotation therapy: a general solution of the inverse problem”, Phys. Med. Biol. 44, 1089- 1104.

Oelfke U. and Bortfeld T. (2001), "Inverse planning for photon and proton beams”, Med. Dosim. 26, 113-124.

Oldham M. and Webb S. (1997), "Intensity-modulated radiotherapy by means of static tomotherapy: A planning and verification study”, Med. Phys. 24, 827-836.

Paliwal B.R., Rommelfanger S. and Das R.K. (1998), "Attenuation characteristics of a new compensator material: Thermo-Shield for high energy electron and photon beams”, Med. Phys. 25(4), 484-487

Pirzkall A., Carol M., Lohr F. et al. (2000), "Comparison of intensity- modulated radiotherapy with conventional conformal radiotherapy for complex- shaped tumors”, Int. J. Radiat. Oncol. Biol. Phys. 48, 1371-1380.

Proimos B.S. (1960), "Synchronous field shaping in rotational megavolt therapy”, Radiology 74(5), 753-757.

Proimos B.S. (1961), "Synchronous protection and field shaping in cyclotherapy”, Radiology 77(4), 591-599.

Proimos B.S. (1963), “New accessories for precise teletherapy with Cobalt-60 units”, Radiology 81, 307-316.

Proimos B.S. (1966), “Beam shapers oriented by gravity in rotational therapy”, Radiology 87(5), 928932. 
Proimos B.S., Tsialas S.P. and Coutroubas S.C. (1966), "Gravity-oriented filters in arc cobalt therapy”, Radiology 87(5), 933-937.

Proimos B.S. and Goldson A.L. (1981), "Dinamic dose shaping by gravity-oriented absorbers for total lymph node irradiation”, Int. J. Radiat. Oncol. Biol. Phys. 7, 973-977.

Proimos B.S. and Danciu C. (1997a), “Photomodelling': a simple conformal radiotherapy technique modeling gravity oriented protectors and photocopying them in the patient”, Proc. World Congress on Medical Physics and Biomedical Engineering and 11th International Conference on Medical Physics (Nice, France, September 1997), Med. Biol. Eng. Comput. 35 (Suppl Part 2), 936

Proimos B.S. and Danciu C. (1997b), “THERA by GOD: THERApy art by a gravity oriented device” Proc. World Congress on Medical Physics and Biomedical Engineering and 11th International Conference on Medical Physics (Nice, France, September 1997), Med. Biol. Eng. Comput. 35 (Suppl Part 2), 1029

Rawlingson J.A. and Cunningham J.R. (1972), “An examination of synchronous shielding in ${ }^{60} \mathrm{Co}$ rotational therapy”, Radiology 102, 667-671.

Redpath A.T., Vickery B.L. and Write D.H. (1976), “A new technique for radiotherapy planning using quadratic programming”, Phys. Med. Biol. 21, 781-791.

Renner W.D., O’Connor T.P. and Bermudez N.M. (1989), “An algorithm for design of beam compensators”, Int. J. Radiat. Oncol. Biol. Phys. 17, 227-234.

Rogers D.W.O. (2002) "Monte Carlo Techniques in Radiotherapy", Physics in Canada, Medical Physics Special Issue, 58(2), 63-70

Schneider W., Bortfeld T. and Schlegel W. (2000), "Correlation between CT numbers and tissue parameters needed for Monte Carlo simulations of clinical dose distribution”, Phys. Med. Biol. 45, 459-478.

Schulz R.J. (1999), "Further improvements in dose distributions are unlikely to affect cure rates", Med. Phys. 26, 1007-1009.

Schulz R.J., Deye J.A. and Hendee W.R. (2001), "Through the preoccupation with new technical developments, physicists have lost sight of the realities of cancer care and statistics”, Med. Phys 28, 2185-2187.

Schulz R.J. and Kagan A.R. (2002), "On the role of intensity-modulated radiation therapy in radiation oncology”, Med. Phys. 29, 1473- 1482.

Sheikh-Bagheri D. and Rogers D.W.O. (2002), "Monte Carlo calculation of nine megavoltage photon beam spectra using the BEAM code”, Med. Phys. 29(3), 391-402.

Shepard D.M., Earl M.A., Li X.A. et al. (2002), "Direct aperture optimization: a turnkey solution for step-and-shoot IMRT”, Med. Phys. 29, 1007-1018. 
Sherouse G.W. (2002), “In regard to Intensity-Modulated Radiotherapy Collaborative Working Group, IJROBP 2001; 51:880-914”, Letter to the Editor, Int. J. Radiat. Oncol. Biol. Phys. 53(4), 1088-1089.

Shimizu K., Yasutake S. and Kondo R. (2003), “A new stilbene with tyrosinase inhibitory activity from Chlorophora excelsa”, Chem. Pharm. Bull. 51(13), 318-319.

Shragge P.C. and Patterson M.S. (1981), "Improved method for the design of tissue compensator", Med. Phys. 8(6), 885-891.

Spirou S.V. and Chui C.S. (1994), "Generation of arbitrary fluence profiles by dynamic jaws or multileaf collimators”, Med. Phys. 21, 1031-1041.

Stein J., Bortfeld T., Doerschel B. et al. (1994), "Dynamic x-ray compensation for conformal radiotherapy by means of multi-leaf collimation”, Radiother. Oncol. 32, 163-173.

Svensson R., Källman P. and Brahme A. (1994), “An analytical solution for the dynamic control of multileaf collimator”, Phys. Med. Biol. 39, 37-61.

Tajiri M., Sunaoka M., Fukumura A. et al. (2004), “A new radiation shielding block material for radiation therapy”, Med. Phys. 31(11), p. 3022- 3023.

Takahashi S. (1965), “Conformation radiotherapy rotation techniques as applied to radiography and radiotherapy of cancer”, Acta Radiol. (Suppl. 242), 1-142.

Thomsen M.S. and Ulso N. (2002), “Attenuation of 4-20 MV X rays by a new compensator material of cement”, Med. Phys. 29(10), 2427-2432.

U.S. National Library of Medicine's Visible Human Project ${ }^{\circledR}$ (2003), http://www.nlm.nih.gov/research/visible

Van Dyk J. (1986), "Broad beam attenuation of cobalt-60 gamma rays and 6-, 18-, and 25-MV x rays by lead”, Med. Phys. 13(1), 105-110.

van Santvoort J.P.C., Binnekamp D., Heijmen B.J.M. et al. (1995), "Granulate stainless steel as compensator material”, Radiother. Oncol. 34, 78-80.

Verhaegen F. and Devic S. (2005), "Sensitivity study for CT image use in Monte Carlo treatment planning”, Phys. Med. Biol. 50, 937-946.

Webb S. (1989), “Optimization of conformal radiotherapy dose distributions by simulated annealing”, Phys. Med. Biol. 34, 1349-1370.

Webb S. (1999), “Conformal intensity-modulated radiotherapy (IMRT) delivered by robotic linacTesting IMRT to the limit?”, Phys. Med. Biol. 44, 1639-1654.

Webb S. (2000), “Conformal intensity-modulated radiotherapy (IMRT) delivered by robotic linacConformality versus efficiency of dose delivery”, Phys. Med. Biol. 45, 1715-1730. 
Webb S. (2001), “Intensity-modulated radiation therapy”, IOP publishing, Bristol and Philadelphia.

Webb S. (2003), “The physical basis of IMRT and inverse planning”, Br. J. Radiol. 76, 678- 689.

Webb S. (2004), “A gravity-oriented-device for IMRT can never rival other IMRT delivery methods”, Phys. Med. Biol. 49, L5-L9.

Weeks K.J., Fraas B.A. and Hutchins K.M. (1988), “Gypsum mixtures for compensator construction”, Med. Phys. 15(3), p. 410- 414.

Weeks K.J. and Sontag M.R. (1990), “3-D dose-volume compensation using nonlinear least- squares regression technique”, Med. Phys. 18(3), 474-480.

Wright K.A., Proimos B.S. and Trumph J.G. (1959), "Physical aspects of two million volt x-ray therapy”, The Surgical Clinics in North America, 39(3), 567-578.

Xia P. and Verhey L.J. (1998), "Multileaf collimator leaf sequencing algorithm for intensity modulated beams with multiple static segments”, Med. Phys. 25, 1424-1434.

Xing L. and Chen G.T.Y. (1996), “Iterative methods for inverse treatment planning”, Phys. Med. Biol. 41, 2107- 2123.

Xu T., Shikhaliev P.M., Al-Ghazi M. et al. (2002), "Reshapable physical modulator for intensity modulated radiation therapy”, Med. Phys. 29(10), 2222-2229.

Yoda K. and Aoki Y. (2003), “A multiportal compensator system for IMRT delivery”, Med. Phys. 30(5), 880-886.

Yu C.X. (1995), "Intensity-modulated arc therapy with dynamic multileaf collimation: an alternative to tomotherapy”, Phys. Med. Biol.40, 1435-1449. 
3D

ABS

BRL

BSF

CNC

CRT

CSDA

CT

DMLC

HESM

HVL

IGT

IMAT

IMB

IMF

IMRT

MC

MCRTS

MLC

MVCT

OAR

PDD

PMMA

PTV

PU

QA

QI

RMS

SAR

SID

SMLC

SSD

TAR

TMR

UW
Three-Dimensional

Acrylonitrile Butadiene Styrene

Brahme, Roos and Lax

Backscatter Factor

Computer Numerical Control

Conformal Radiation Therapy

Continuous-slowing-down approximation

Computed Tomography

Dynamic MLC

High Energy Simulation Module

Half-Value Layer

Image Guided Therapy

Intensity Modulated Arc Therapy

Intensity Modulated Beams

Intensity Modulated Fields

Intensity-Modulated Radiation Therapy

Monte Carlo

Monte Carlo Radiotherapy Simulator

Multileaf Collimator

Megavolt Computed Tomography

Organ At Risk

Percentage Depth Dose

Polymethyl methacrylate

Planning Target Volume

Polyurethane

Quality Assurance

Quality Index

Root-mean-square

Scatter-air ratio

Source-to-isocenter distance

Static MLC

Source-to-surface distance

Tissue-air ratio

Tissue-maximum ratio

University of Wisconsin 
APPENDIX A

\section{THE DERIVATION OF THE DOSE CALCULATION UNDER A PARTIAL ATTENUATOR}

The dose at point $\mathrm{C}$ in a phantom under a partial attenuator $\left[\mathrm{D}\left(\mathrm{R}, \mathrm{W}_{0} ; \mathrm{w}\right)\right]$, expressed as a fraction of the dose at the same point in the phantom in the absence of absorber $\left[D\left(R, W_{0}\right)\right]$, can be calculated by subtracting the unattenuated dose at point $C$ for field size $w[D(R, w)]$ from the unattenuated dose at point $C$ for field size $W_{0}\left[D\left(R, W_{0}\right)\right]$ and adding to the difference the dose at point $\mathrm{C}$ for the fully covered field size $\mathrm{w}[\mathrm{D}(\mathrm{d}, \mathrm{w} ; \mathrm{w})]($ El-Khatib et al. 1988, Khan 1994). The schematic representation of the method is depicted in Fig. A-1.

So, the dose in the phantom under the partial attenuator can be calculated with a manipulation of two open beams and the beam in which the attenuator covers the full radiation field:

$$
\frac{\mathrm{D}\left(\mathrm{R}, \mathrm{W}_{0} ; \mathrm{w}\right)}{\mathrm{D}\left(\mathrm{R}, \mathrm{W}_{0}\right)}=\frac{\mathrm{D}\left(\mathrm{R}, \mathrm{W}_{0}\right)-\mathrm{D}(\mathrm{R}, \mathrm{w})+\mathrm{D}(\mathrm{R}, \mathrm{w} ; \mathrm{w})}{\mathrm{D}\left(\mathrm{R}, \mathrm{W}_{0}\right)},
$$

with

$$
\begin{aligned}
& \mathrm{D}\left(\mathrm{R}, \mathrm{W}_{0}\right)=\mathrm{D}[\text { Fig. } \mathrm{A}-1(\mathrm{~b})]=\mathrm{D}_{\mathrm{fs}} \cdot \operatorname{BSF}\left(\mathrm{W}_{0}\right) \cdot \operatorname{PDD}\left(\mathrm{R}, \mathrm{W}_{0}\right) / 100, \\
& \mathrm{D}(\mathrm{R}, \mathrm{w})=\mathrm{D}[\text { Fig. A-1(c) }]=\mathrm{D}_{\mathrm{fs}} \cdot \operatorname{BSF}(\mathrm{w}) \cdot \operatorname{PDD}(\mathrm{R}, \mathrm{w}) / 100,
\end{aligned}
$$


(a)

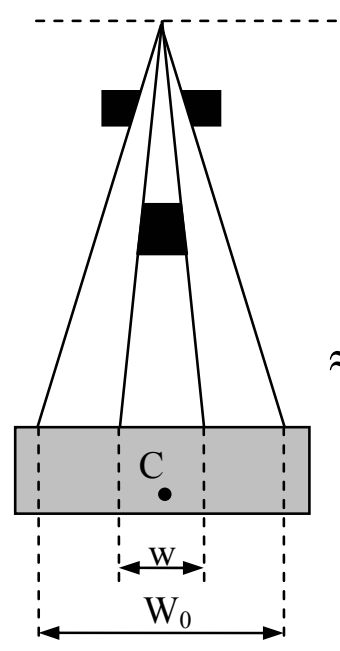

$\mathrm{D}\left(\mathrm{R}, \mathrm{W}_{0} ; \mathrm{w}\right)$ (b)

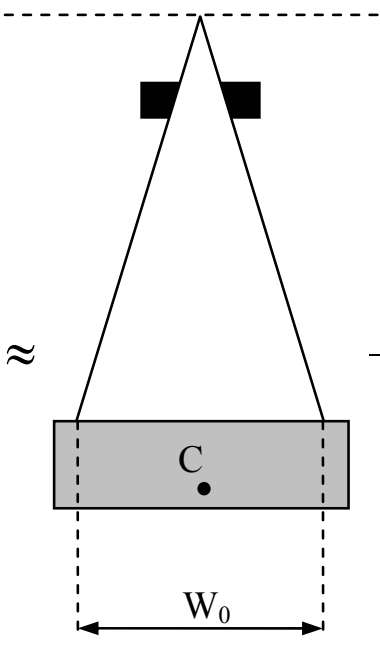

$\mathrm{D}\left(\mathrm{R}, \mathrm{W}_{0}\right)$ (c)

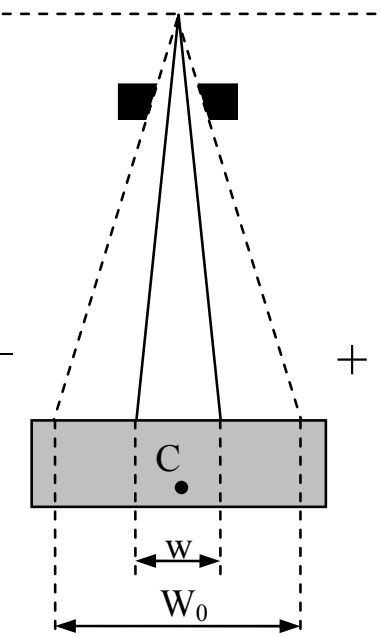

$\mathrm{D}(\mathrm{R}, \mathrm{w})$ (d)

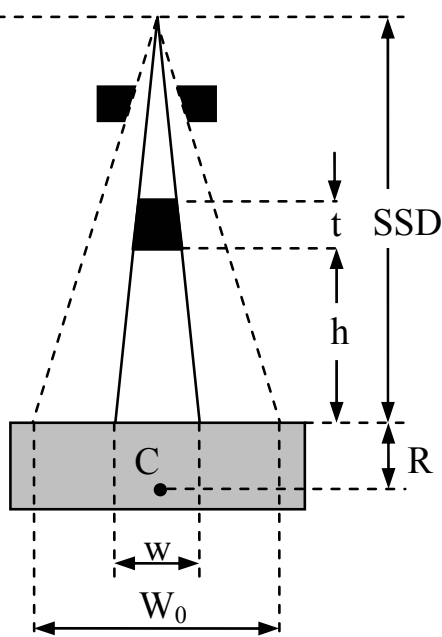

$\mathrm{D}(\mathrm{R}, \mathrm{w} ; \mathrm{w})$

FIGURE A-1. Schematic representation of the method of the dose calculation under a partial attenuator. Part (a) represents the partial attenuator $\mathrm{w}$ in the beam $W_{0}$ [dose at $\mathrm{C}$ is $D(R, W ; w)$ ], part (b) represents the open field $W_{0}$ with the dose at $\mathrm{C}$ as $D\left(R, W_{0}\right)$, part (c) is for the open field $w$ with the dose at $\mathrm{C} D(R, w)$, and part (d) represents the field $w$ fully covered by the attenuator with the resulting dose at $\mathrm{C} D(R, w ; w)$.

and

$$
\mathrm{D}(\mathrm{R}, \mathrm{w} ; \mathrm{w})=\mathrm{D}[\text { Fig. A-1 }(\mathrm{d})]=\mathrm{D}_{\mathrm{fs}} \cdot \mathrm{F} \cdot \mathrm{BSF}(\mathrm{w}) \cdot \operatorname{PDD}(\mathrm{R}, \mathrm{w}) / 100,
$$

where $\mathrm{D}_{\mathrm{fs}}$ is the dose at the point $\mathrm{C}$ in a free space, PDD represents the standard percentage depth dose at depth $\mathrm{R}$ for a given field size and $\operatorname{BSF}\left(\mathrm{W}_{0}\right)$ and $\operatorname{BSF}(w)$ are the backscatter factors for field sizes $\mathrm{W}_{0}$ or $\mathrm{w}$, respectively. Transmitted fraction $\mathrm{F}$ for fully covered radiation field is defined as

$$
\mathrm{F}=\mathrm{D}(\mathrm{R}, \mathrm{w} ; \mathrm{w}) / \mathrm{D}(\mathrm{R}, \mathrm{w})
$$

Thus, the relative dose for partially attenuated beam can be calculated by a simple analytical method:

$$
\frac{\mathrm{D}\left(\mathrm{R}, \mathrm{W}_{0} ; \mathrm{w}\right)}{\mathrm{D}\left(\mathrm{R}, \mathrm{W}_{0}\right)}=1-\frac{\operatorname{PSF}(\mathrm{w})}{\operatorname{PSF}\left(\mathrm{W}_{0}\right)} \frac{\operatorname{PDD}(\mathrm{d}, \mathrm{w})}{\operatorname{PDD}\left(\mathrm{d}, \mathrm{W}_{0}\right)}(1-\mathrm{F})
$$

Another derivation, leading to the same (or interchangeable) calculation expression of the dose at the center of the shielded zone was given by Rawlingson and Cunningham (1972) and used and modified by Fiorino et al. (1995) and Danciu (2001).

If the absorber is assumed to transmit a constant fraction $\mathrm{F}$ of the incident primary fraction, then the dose will consist of: 
- a contribution due to primary radiation, given by $T A R(R, 0) \cdot F$, where $T A R(R, 0)$ is the zero area tissue-air ratio;

- a contribution of magnitude $\operatorname{SAR}(\mathrm{R}, \mathrm{w}) \cdot \mathrm{F}$ due to scatter from the shielded region, where $\operatorname{SAR}(\mathrm{R}, \mathrm{w})$ is the scatter-air ration for a beam of dimensions $\mathrm{w}$;

- a contribution due to scatter from the irradiated region, given by SAR(R, W0)$\operatorname{SAR}(\mathrm{R}, \mathrm{w})$, where $\mathrm{SAR}(\mathrm{R}, \mathrm{W} 0)$ is the scatter-air ratio for the entire beam.

The dose at depth $\mathrm{R}$ in the shielded region, expressed as a fraction of the dose at the same point in the phantom in the absence of the absorber, can be written as

$$
\frac{\mathrm{D}\left(\mathrm{R}, \mathrm{W}_{0} ; \mathrm{w}\right)}{\mathrm{D}\left(\mathrm{R}, \mathrm{W}_{0}\right)}=\frac{\operatorname{TAR}\left(\mathrm{R}, \mathrm{W}_{0} ; \mathrm{w}\right) \cdot \mathrm{D}_{\mathrm{fs}}}{\mathrm{TAR}\left(\mathrm{R}, \mathrm{W}_{0}\right) \cdot \mathrm{D}_{\mathrm{fs}}}
$$

where $\operatorname{TAR}\left(\mathrm{R}, \mathrm{W}_{0} ; \mathrm{w}\right)$ is the tissue-air-ratio at depth $\mathrm{R}$ in phantom for field of dimensions $\mathrm{W}_{0}$ with the presence of absorber and $\operatorname{TAR}\left(\mathrm{R}, \mathrm{W}_{0}\right)$ is the tissue-air-ratio at depth $\mathrm{R}$ in phantom for the same field size in the absence of absorber.

Taking into account all constituents of the total dose, Eq. A-7 can be rewritten as following:

$$
\frac{\mathrm{D}\left(\mathrm{R}, \mathrm{W}_{0} ; \mathrm{w}\right)}{\mathrm{D}\left(\mathrm{R}, \mathrm{W}_{0}\right)}=\frac{\mathrm{TAR}(\mathrm{R}, 0) \cdot \mathrm{F}+\mathrm{SAR}(\mathrm{R}, \mathrm{w}) \cdot \mathrm{F}+\left[\mathrm{SAR}\left(\mathrm{R}, \mathrm{W}_{0}-\mathrm{SAR}(\mathrm{R}, \mathrm{w})\right]\right.}{\mathrm{TAR}\left(\mathrm{R}, \mathrm{W}_{0}\right)} .
$$

Expressing SARs through TARs

$$
\begin{aligned}
& \operatorname{SAR}\left(\mathrm{R}, \mathrm{W}_{0}\right)=\operatorname{TAR}\left(\mathrm{R}, \mathrm{W}_{0}\right)-\operatorname{TAR}(\mathrm{R}, 0), \\
& \operatorname{SAR}(\mathrm{R}, \mathrm{w})=\operatorname{TAR}(\mathrm{R}, \mathrm{w})-\operatorname{TAR}(\mathrm{R}, 0)
\end{aligned}
$$

and substituting them in the Eq. A-8, the final expression will be as follows:

$$
\frac{\mathrm{D}\left(\mathrm{R}, \mathrm{W}_{0} ; \mathrm{w}\right)}{\mathrm{D}\left(\mathrm{R}, \mathrm{W}_{0}\right)}=1-\frac{\mathrm{TAR}(\mathrm{R}, \mathrm{w})}{\mathrm{TAR}\left(\mathrm{R}, \mathrm{W}_{0}\right)}(1-\mathrm{F})
$$

Using well known relations between TAR, TMR, BSF and PDD (Khan 1994, Khan et al. 1980), the relative dose in one point behind an absorber can be expressed also like

$$
\frac{\mathrm{D}\left(\mathrm{R}, \mathrm{W}_{0} ; \mathrm{w}\right)}{\mathrm{D}\left(\mathrm{R}, \mathrm{W}_{0}\right)}=1-\frac{\mathrm{BSF}(\mathrm{w})}{\mathrm{BSF}\left(\mathrm{W}_{0}\right)} \frac{\mathrm{TMR}(\mathrm{R}, \mathrm{w})}{\mathrm{TMR}\left(\mathrm{R}, \mathrm{W}_{0}\right)}(1-\mathrm{F}) .
$$

It can also coincide with the Eq. A-6.

As discussed in the work of El-Khatib et al. (1988), the results of the above presented approximation of the dose calculation of the partially attenuated field are closer to the 
measured data than the dose calculations accounting only for primary beam attenuating. Even better calculation results can be achieved, if the transmission factor $\mathrm{F}$ for the fully covered field is calculated not as simple exponential $\exp \left(-\mu_{\text {eff }} \mathrm{t}\right)$, but accounting additionally for the first scatter and beam hardening effects in the attenuator and phantom. 
APPENDIX B

\section{CALCULATIONS OF A SHAPER FILTRATION CHORD (RAY-SHAPER INTERSECTION)}

In the both methods of shapers parameters calculations (See Chapter 4, sect. 4.3) the shaper's thickness, which is a ray filtration chord, has to be calculated for every gantry angle. For calculations of beam paths in the shapers the following approach has been used. The illustration of the geometrical representation of the problem is shown in Fig. B-1. Only one shaper (right one, labeled as second shaper) is shown here, the first one is omitted for the clarity. The coordinates of the center of the shaper are $\left(x_{\mathrm{sh} 2}, \mathrm{y}_{\mathrm{sh} 2}, \mathrm{z}_{\mathrm{sh} 2}\right)$. The coordinates of the point I of the phantom, for which the chord filtration will be calculated, are represented by a vector $\mathbf{r}_{\mathbf{p}}=\left(\mathrm{x}_{\mathrm{p}}, \mathrm{y}_{\mathrm{p}}, \mathrm{z}_{\mathrm{p}}\right)$ and that of the source by a vector $\mathbf{r}_{\mathrm{s}}=\left(\mathrm{x}_{\mathrm{s}}, \mathrm{y}_{\mathrm{s}}, \mathrm{z}_{\mathrm{s}}\right)$. Hereinafter, $\mathrm{x}$ dimension will not be considered because the point of max intensity will bes chosen in the YZ plane that passes through the isocenter.

A parametric presentation of a ray, passing through point I, in two dimensions is:

$$
\begin{aligned}
& y=y_{p}+b_{y} \cdot t \\
& z=z_{p}+b_{z} \cdot t
\end{aligned}
$$




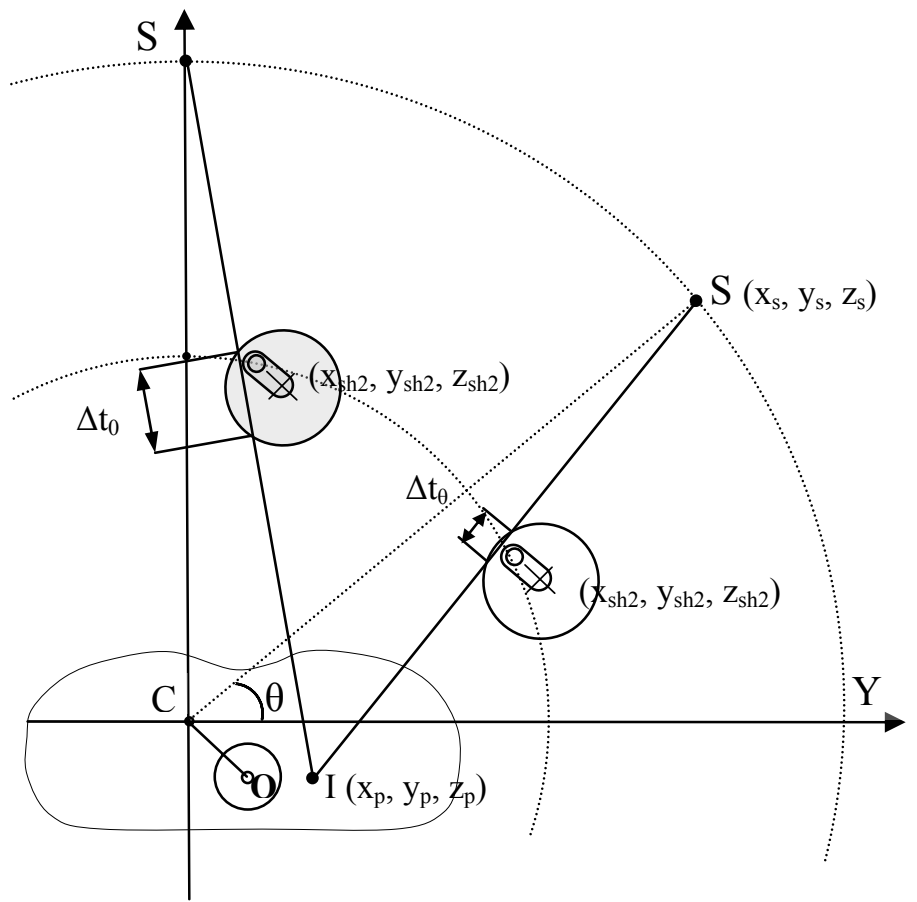

FIGURE B-1. Illustration of shapers motion and corresponding change of filtration chord $\Delta \mathrm{t}_{\theta}$ with gantry angle $\theta$ for a given point $I$ with coordinates $\left(x_{p}, y_{p}, z_{p}\right)$. The isocenter is labeled by $\mathrm{C}$, the center of the OAR by $\mathrm{O}$, and the source is represented by $\mathrm{S}$. The coordinates of the right shaper's center are $\left(\mathrm{x}_{\mathrm{sh} 2}, \mathrm{y}_{\mathrm{sh} 2}, \mathrm{z}_{\mathrm{sh} 2}\right)$. The left shaper is avoided for clarity.

where the components $\left(\mathrm{b}_{\mathrm{y}}, \mathrm{b}_{\mathrm{z}}\right)$ are the directions' cosines

$$
\begin{aligned}
& b_{y}=\frac{y_{s}-y_{p}}{n} \\
& b_{z}=\frac{z_{s}-z_{p}}{n},
\end{aligned}
$$

which are normalized by

$$
n=\sqrt{\left(y_{s}-y_{p}\right)^{2}+\left(z_{s}-z_{p}\right)^{2}} .
$$

The cross section of the cylinder is described by the circle's equation: $\left(y-y_{\operatorname{sh} 2}\right)^{2}+\left(z-z_{s h 2}\right)^{2}=R^{2}$. The distance between the points of intersection of a ray and circle is the chord size. So, solving the equation

$$
\left(\mathrm{y}_{\mathrm{p}}+\mathrm{b}_{\mathrm{y}} \mathrm{t}-\mathrm{y}_{\mathrm{sh} 2}\right)^{2}+\left(\mathrm{z}_{\mathrm{p}}+\mathrm{b}_{\mathrm{z}} \mathrm{t}-\mathrm{z}_{\mathrm{sh} 2}\right)^{2}=\mathrm{R}^{2}
$$

regarding to $t$ and finding two points of the ray-shaper intersection, we will find the chord length $\Delta t=\left|t_{1}-t_{2}\right|$. Rewriting the equation in a form of $A t^{2}+B t+C=0$, the solution is

$$
\Delta t=\frac{\sqrt{B^{2}-4 A C}}{A}
$$


where

$$
\begin{aligned}
& A=b_{y}^{2}+b_{z}^{2}, \\
& \mathrm{~B}=2 \mathrm{~b}_{\mathrm{y}} \mathrm{y}_{\mathrm{psh} 2}-2 \mathrm{~b}_{\mathrm{z}} \mathrm{z}_{\mathrm{psh} 2}, \\
& \mathrm{C}=\mathrm{y}_{\mathrm{psh} 2}^{2}+\mathrm{z}_{\mathrm{psh} 2}^{2}-\mathrm{R}^{2}
\end{aligned}
$$

where, in its turn

$$
\mathrm{y}_{\mathrm{psh} 2}=\mathrm{y}_{\mathrm{p}}-\mathrm{y}_{\mathrm{sh} 2}
$$

and

$$
\mathrm{z}_{\mathrm{psh} 2}=\mathrm{z}_{\mathrm{p}}-\mathrm{z}_{\mathrm{sh} 2}
$$

The same is also valid for the left shaper. Half of the gantry rotation time the point I is under filtration of one of the shapers and the other half time under the filtration of the other shaper. However, due to symmetry of the setup the times the point is under filtration and the average filtration chords of both shapers are equal. So, the total filtration can be calculated as a double filtration of one shaper. 